\title{
Nanoscale probing of single synapse function and BDNF Cell-to-Cell transfer
}

\author{
Dissertation \\ for the award of the degree \\ "Doctor rerum naturalium" \\ of the Georg-August-University Göttingen \\ within the Neurosciences doctoral program \\ of the Georg-August University School of Science (GAUSS)
}

submitted by

Markus Andreas Stahlberg

from Heidelberg, Germany

Göttingen 2016 


\title{
Thesis Committee
}

\author{
Camin Dean, PhD Department of Trans-Synaptic Signaling \\ (Reviewer) \\ European Neuroscience Institute, Göttingen \\ Prof. Dr. Stefan W. Hell \\ Department of NanoBiophotonics \\ (Reviewer) \\ Max Planck Institute for Biophysical Chemistry, \\ Göttingen
}

Prof. Dr. Dr. Detlev Schild

Institute of Neurophysiology and Cellular Biophysics

University Medical Center Göttingen

\section{Members of the Examination Board}

\author{
Camin Dean, PhD Department of Trans-Synaptic Signaling \\ (Reviewer) \\ European Neuroscience Institute, Göttingen \\ Prof. Dr. W. Stefan Hell \\ Department of NanoBiophotonics \\ (Reviewer) \\ Max Planck Institute for Biophysical Chemistry, \\ Göttingen
}

\section{Further members of the Examination Board}

Prof. Dr. Dr. Detlev Schild

Dr. Sebastian Kügler

Prof. Dr. Klaus-Armin Nave

Prof. Dr. Thomas Dresbach
Institute of Neurophysiology and Cellular Biophysics University Medical Center Göttingen

Department of Neurology

University Medical Centre Göttingen

Department of Neurogenetics

Max Planck Institute for Experimental Medicine, Göttingen

Department of Anatomy and Embryology University Medical Centre Göttingen 


\section{Dedication}

I dedicate this thesis to my mum and my brothers, the people who I am indebted to the most and who had the most substantial influence on the person I became. 


\section{Abstract}

Brain-derived neurotrophic factor (BDNF) is one of the key modulator/mediator molecules for synaptic plasticity in the adult nervous system and also coordinates neural development, survival, differentiation and axon growth during development in the central and the peripheral nervous system. The understanding of our brain critically depends on the detailed understanding of the regulatory mechanisms of molecules like BDNF.

Recent research has implicated BDNF in synapse-specific strengthening of active synapses, but if BDNF is recruited specifically to active synapses to modulate their function is not known. Additionally, it is debated whether BDNF is released from post- or presynaptic sites and if BDNF affects exclusively neurons, or other cell types, like astrocytes, in addition.

Here we propose and investigate a novel strategy to achieve focal stimulation of neurons using optogenetics, with the ultimate goal to study the influence of activity on the recruitment and release of the neurotrophic factor BDNF. We demonstrate the utility of current optogenetic tools to achieve highly focal depolarization and further examined a proof-of-principle of nanoscale optogenetic activation using an initial macroscale approach.

Using dissociated and organotypic hippocampal cultures from the rat, in which we co-express BDNF-mRFP1 and a cytosolic fluorophore to identify the cell of origin, we tested the influence of focal optogenetic activation of specific sites on intracellular BDNF trafficking and further extended the study to investigate the intercellular transfer of BDNF to neighboring cells. We found that BDNF was preferentially taken up by astrocytes and provide evidence for BDNF-mediate physiological effects on the astrocytic population. 


\section{Table of Contents}

\section{Abstract}

List of figures and tables $\quad 1$

Abbreviations 3

2. Introduction $\quad 6$

The nervous system and synaptic transmission $\quad 6$

$\begin{array}{lr}\text { Brain-derived neurotrophic factor (BDNF) } & 10\end{array}$

BDNF signaling dysregulation is involved in certain pathologies and disorders $\quad 15$

$\begin{array}{ll}\text { Techniques to probe synaptic plasticity } & 16\end{array}$

3. Materials and Methods $\quad \mathbf{2 1}$

3.1. Suppliers $\quad 21$

3.2. Molecular biology 21

$\begin{array}{ll}3.2 .1 \text { Vectors } & 21\end{array}$

3.2.2. Plasmid amplification and molecular cloning 23

3.3. Cell cultures 26

$\begin{array}{ll}\text { 3.3.1. Dissociated primary hippocampal cultures } & 27\end{array}$

3.3.2. Organotypic hippocampal brain slices (OHBS) 30

3.3.3. HEK 293 cultures $\quad 32$

3.4. Experiments \& data acquisition 33

3.4.1. Immunocytochemistry (ICC) 33

3.4.2. Microscopy $\quad 35$

3.4.3. Optogenetics and electrophysiology 36

$\begin{array}{ll}\text { 4. Results } & 41\end{array}$

4.1. Nanoscale Optogenetics 41

Channelrhodopsin characterization $\quad 41$

Efficient photocurrent reduction by intensified $594 \mathrm{~nm}$ illumination 48

Effects of strong 594 laser power on fast-photocycle variants 49

Focal optogenetical stimulation of neuronal processes $\quad 50$

Dual wavelength illumination for focal depolarization on the macro scale $\quad 51$

Different illumination approaches for reaching focal photostimulation in the nanoscale $\quad 52$

4.2. BDNF-mRFP1 migration and cell-to-cell transfer 53

Effects of focal optogenetic stimulation on BDNF trafficking 53

BDNF-mRFP1 is released by neurons and transferred to nearby cells 54

Transferred BDNF-mRFP1 is taken up by target cells $\quad 57$

Assessing transfer directionality of BDNF-mRFP1 in organotypic hippocampal slices 
BDNF-mRFP1 uptake by different cell types

Subcellular localization of BDNF-mRFP1 taken up by astrocytes and neurons

Expression of TrkB in neurons increases neuronal and decreases astrocytic

BDNF-mRFP1 uptake

Influence of BDNF-mRFP1 on astrocyte abundance in dissociated hippocampal cultures

5. Discussion

5.1 Nanoscale optogenetics

Functional characterization and testing of channels

Electrophysiological and visual detection of focal stimulation

5.2 BDNF

Influence of focal light stimulation on BDNF recruitment

BDNF cell-to-cell transfer

Release of ProBDNF versus mature BDNF and physiological consequences for astrocytes

Conclusion and future directions

Acknowledgments 


\section{List of figures and tables}

Figure 1: ChR2 photocycle

Figure 2: Custom modified microscope light path, photomasks and experimental approach for optogenetic and electrophysiological experiments.

Figure 3: Illumination evoked responses of light-gated channels with fast photocycles.

Figure 4: Illumination evoked responses of light-gated channels with slow photocycles (step-function opsins) and the eNPAC construct.

Figure 5: Reduction of 488 and 405 nm evoked photocurrents in ChR2 C128A/H134R/T159C.

Figure 6: Photocurrent reduction of 488 and $405 \mathrm{~nm}$ evoked currents in CoChR C108S and C108S/D136A mutants.

Figure 7: Effect of higher $594 \mathrm{~nm}$ laser intensity on fast photocycle variants.

Figure 8: Electrophysiological recordings and visual detection of focally stimulated areas.

Figure 9: Focal stimulation proof of concept at the macro scale.

Figure 10: Illumination approaches for focal photostimulation using light-gated ion channels/pumps.

Figure 11: Fluorescent BDNF puncta trafficking and effects of focal optogenetic stimulation.

Figure 12: Map2 and GFAP immunocytochemical labeling of a PO derived dissociated hippocampal culture expressing BDNF-mRFP1.

Figure 13: Loss of BDNF-mRFP1 fluorescence in culture through standard fixation and permeabilization procedures for ICC.

Figure 14: Assessment of fluorophore contribution to transfer.

Figure 15: Fluorophore independent transfer of BDNF.

Figure 16: Validation of preconjugated primary and secondary antibody detection of extracellular antigens.

Figure 17: Transferred BDNF-mRFP1 is internalized.

Figure 19: AAV-mediated overexpression of EGFP and BDNF-mRFP1-P2A-ECFP in the CA3 and CA1regions of organotypic hippocampal brain slice (OHBS) cultures.

Figure 18: Spread of BDNF-mRFP1 signal and transfer to nearby cells in OHBS.

Figure 20: Injection of purified BDNF-mRFP1-P2A-EGFP or mRFP1-P2A-EGFP AAV6 to CA 3 and $C A 1$ in OHBS.

Figure 21: Assessment of BDNF-mRFP1 uptake by different cell types regularly found in dissociated hippocampal cultures at DIV14.

Figure 22: AAV-mediated live labeling of neurons and astrocytes.

Figure 23: ICC validation of subcellular localization marker constructs for EEA1, Rab5a, Rab7 and Lamp1.

Figure 24: Colocalization of taken up BDNF-mRFP1 with subcellular marker proteins. 
Figure 25: Verification of exclusive TrkB receptor expression in neurons.

Figure 26: TrkB overexpression in neurons increases BDNF-mRFP1 uptake.

Figure 27: TrkB overexpression in neurons increases BDNF-mRFP1 uptake by neighboring neurons and reduces BDNF-mRFP1 found in astrocytes.

Figure 28: Changes in BDNF-mRFP1 uptake due to TrkB overexpression.

Figure 29: Influence of stimulated BDNF release on astrocyte abundance. 71

Figure 30: Influence of BDNF abundance on GFAP immunoreactivity. 


\begin{tabular}{|c|c|}
\hline${ }^{\circ} \mathrm{C}$ & degree Celsius \\
\hline aa & amino acids \\
\hline AAV & adeno-associated virus \\
\hline AF488 & Alexa Fluor 488 \\
\hline AMPA receptor & $\alpha$-amino-3-hydroxy-5-methyl-4-isoxazolepropionic acid receptor \\
\hline ApE & A plasmid editor \\
\hline APV (AP5) & 2-amino-5-phosphonopentanoic acid \\
\hline BDNF & Brain-derived neurotrophic factor \\
\hline $\mathrm{BIOS}$ & basic input/output system \\
\hline bp & base pair \\
\hline C1V1 & channelrhodopsin chimera o ChR1 and VChR1 \\
\hline CA1 and CA3 & Cornu Ammonis area 1 and 3 \\
\hline cat. no. & Catalogue number \\
\hline CatCh & calcium translocating channelrhodopsin \\
\hline CDS & coding DNA sequence \\
\hline ChR & channelrhodopsin \\
\hline cLTP & chemical LTP \\
\hline $\mathrm{cm}$ & centimeter \\
\hline CMV & promotor derived from cytomegalovirus \\
\hline CNQX & 6-cyano-7-nitroquinoxaline-2,3-dione \\
\hline $\mathrm{CO} 2$ & carbon dioxide \\
\hline DCVs & dense core vesicles \\
\hline $\mathrm{dH} 2 \mathrm{O}$ & deionized water \\
\hline DIV & day in vitro \\
\hline DMEM & Dulbecco's modified Eagle media \\
\hline DMSO & dimethyl sulfoxide \\
\hline DNA & deoxyribonucleic acid \\
\hline DPBS & Dulbecco's Phosphate-Buffered Saline \\
\hline DPSS & diode pumped solid state \\
\hline Dr. & Doctor \\
\hline e.g. & for example, exempli gratia \\
\hline E18 or 19 & embryonical day 18 or 19 \\
\hline ECFP & enhanced cyan fluorescent protein \\
\hline EDTA & ethylenediaminetetraacetic acid \\
\hline EGFP & enhanced green fluorescent protein \\
\hline EMCCD & electron multiplying charge-coupled device \\
\hline et al & and others, et alli \\
\hline EYFP & enhanced yellow fluorescent protein \\
\hline FBS & fetal bovine serum \\
\hline FRAP & fluorescence recovery after photobleaching \\
\hline g & gram \\
\hline GABA & gamma-aminobutyric acid ( $\nu$-aminobutyric acid) \\
\hline GFAP & glial fibrillary acidic protein \\
\hline HBSS & Hank's balanced salt solution \\
\hline HEK & human embryonic kidney \\
\hline $\mathrm{Hz}$ & hertz \\
\hline i.e. & that is, id est \\
\hline Iba1 & ionized calcium-binding adapter molecule 1 \\
\hline ITR & internal tandem repeat \\
\hline $\mathrm{kDa}$ & kilodalton \\
\hline $\mathrm{kHz}$ & kilohertz \\
\hline
\end{tabular}




\begin{tabular}{|c|c|}
\hline LB & lysogeny broth \\
\hline LSM & laser scanning microscope \\
\hline LSSmOrange & large stokes shift mOrange \\
\hline LTD & long term depression \\
\hline LTP & long term potentiation \\
\hline M & molar / molar concentration \\
\hline Map2 & microtubule-associated protein 2 \\
\hline MEM & minimal essential medium \\
\hline $\min$ & minutes \\
\hline $\mathrm{ml}$ & milliliter \\
\hline $\mathrm{mm}$ & millimeter \\
\hline $\mathrm{mM}$ & millimolar \\
\hline mol & mole \\
\hline mOsm & milliosmole \\
\hline mRFP1 & monomeric red fluorescent protein 1 \\
\hline mRNA & messenger ribonucleic acid \\
\hline $\mathrm{mV}$ & millivolt \\
\hline $\mathrm{mW}$ & milliwatt \\
\hline$M \Omega$ & megaohm \\
\hline $\mathrm{nA}$ & nanoampere \\
\hline $\mathrm{NaOH}$ & sodium hydroxide \\
\hline ng & nanogram \\
\hline NGF & nerve growth factor \\
\hline $\mathrm{Nh} 4 \mathrm{Cl}$ & ammonium chloride \\
\hline $\mathrm{nl}$ & nanoliter \\
\hline $\mathrm{nM}$ & nanomolar \\
\hline $\mathrm{NpHR}$ & halorhodopsin \\
\hline NT-3/4/5 & neurotrophin-3/4/5 \\
\hline$\varnothing$ & diameter \\
\hline OD & optical density \\
\hline $\mathrm{OD}_{600}$ & optical density determined using the absorption at $600 \mathrm{~nm}$ \\
\hline OHBS & organotypic hippocampal brain slices \\
\hline OPSL & optically pumped semiconductor laser \\
\hline ORF & open reading frame \\
\hline P0 or P4 & postnatal day 0 or 4 \\
\hline p75NTR & p75 neurotropin receptor \\
\hline $\mathrm{pA}$ & piccoampere \\
\hline PBS & phosphate-buffered saline \\
\hline PEI & polyethylenimine \\
\hline PFA & paraformaldehyde \\
\hline Ph.D. & Philosophiae Doctor (doctor of philosophy) \\
\hline $\mathrm{p}_{\mathrm{i}}$ & injection pressure \\
\hline PSD-95 & postsynaptic density -95 \\
\hline rcf & relative centrifugal force \\
\hline RESOLFT & reversible saturable optical (fluorescence) transitions \\
\hline RFP & red fluorescent protein \\
\hline rpm & revolutions per minute \\
\hline S & seconds \\
\hline SDS & Sodium dodecyl sulphate \\
\hline SFO & step-function opsin \\
\hline SSFO & stable step-function opsin \\
\hline STED & stimulated emission depletion \\
\hline syn & synapsin \\
\hline
\end{tabular}


Syt

TAE

TGN

TIRF

TrkB

TrkB Ig-G

TTX

UV

VChR1

W

$\mu \mathrm{g}$

$\mu l$

$\mu \mathrm{m}$

$\mu \mathrm{s}$ synaptotagmin

tris base, acetic acid and EDTA buffer

trans-Golgi network

total internal reflection fluorescence

tropomyosin receptor kinase $B$

TrkB Immunogobulin G

tetrodotoxin

ultra violet

cation-conducting channelrhodopsin (VChR1) from Volvox carteri

watt

microgram

microliter

micrometer

microsecond 


\section{Introduction}

\section{The nervous system and synaptic transmission}

The evolution of life is without question one of the most fascinating phenomena that took place in the short existence of our world. Though it is not yet clear whether this incident is unique or part of a general more fundamental rule of nature, it gave rise to a complex multitude of diverse life forms during the time course of evolution, with the cell constituting the smallest and most basic unit. With growing organisms and organization, division of labor and function presented an opportunity for efficiency. This division consequently resulted in complex biological systems on their own, working in close communion. Because it was necessary to provide coordinated responses to environmental conditions and work in synchrony, one of these organizational subdivisions acquired the function of a central communication center, collecting information through sensory afferents, processing their relevance and giving appropriate directives to other organizational subdivisions. Thus it is orchestrating vital cooperation for the physiological maintenance of its multicellular society and motility. This communication/coordination center is known as nervous system.

While some organisms bet on massive reproduction in the game of evolution, some did on complexity, persistence and adaptation. Complex environmental conditions demanded complex and adapted responses and thus require complex and well-reasoned behavior, which gave rise to the development of more complex nervous systems in some organisms. This demand in complexity consequently resulted in larger and more powerful processing abilities. Integrated with its sensory perceptions of the surrounding world this created biological representations of operating systems, personalities. Within specific parameters these identities have control over simple functions of the organism and the neuronal infrastructure, while this behavior and more basic functions are monitored and emotionally tuned/guided by a subconscious BIOS. To understand the purpose and the consequences of actions is one of the primary functions of this personality, which is expressed by curiosity and a desire to understand the context of its surroundings. Interestingly this curiosity is also reflected toward its own existence and more ironically, also toward understanding the origin of such curiosity. The endeavor to study and understand the origin of behavior and perceptual reality is called Neuroscience. As we well know nowadays the origin of a perceptual personality is the interaction of and within complex neuronal networks. It is an amusing fact that our personality, which is the functional consequence of a complex interaction of cells, is at least in part intrigued by its own function.

However, fundamental understanding about our own biology is also vital to fight pathologies and help us to adapt better to our environment by learning from a complex system that took billions of years to evolve to its current state. And while humans have not been significantly changing biologically within the last millennia, because such a process would take much longer, the human society is adapting through a process of metaevolution, where individuals learn and adapt according to information that exists only in the interaction of the society's members, just like in a neuronal 
network. Information, that depends extensively on the knowledge that we have about fundamental biological processes; an endeavor that is of epic proportions. Under the search ["nervous system" or neuron], PubMed lists 956,665 article entries (date $28^{\text {th }}$ of March 2016), with currently more than 30,000 new articles every year; a collective amount of information that is beyond the processing capabilities of a single individual. And it will take the combined human-network effort of many more generations to clarify all its functions.

In order to understand the origin of perception, complex phenomena like learning and memory and consequently the cause of disorders affecting lives, researches were trying to reduce complicated macroscopic biological processes to their most fundamental units, the neurons. The human central nervous system consists of an estimated 85 billion $(85,000,000,000)$ neurons, but roughly 10 times more glia cells (Kandel, 2001; Williams and Herrup, 1988). Just as the heart is vital for the brain to function, neurons would not be able to operate without supporting neuronal functions through myelination (oligodendrocytes), biochemical support and homeostasis (astrocytes), immune defense (microglia) and formation of the ventricular system and production of cerebrospinal fluid (ependymal cells). Recent results indicate that some of these cells play more active roles in the regulation of synaptic transmission. Though, neurons are still considered to be the most substantial protagonists in the formation of human perception, dysregulations in any of the above-mentioned processes can affect the whole system dramatically.

Only $1 / 7^{\text {th }}, 10-12$ billion neurons, actually belong to the telenchephalic brain region (Williams and Herrup, 1988), which forms the Cerebrum, including the cerebral cortex, and subcortical areas like the hippocampus and basal ganglia - structures that have been identified to contribute most substantially to complex perceptual processes. However, as we know, this number is not much more than a mere fun fact. Size, weight and number of neurons do not necessarily correlate with intelligence or processing power (Cobb, 1965; Hechst, 1932). The secret lies within the interconnection of neurons and the complex neuronal networks, that are formed this way. These connections need to be studied and understood.

The first basic achievements in understanding the brain's functions depended on the invention of appropriate investigation methods, like the use of lenses in optics to build microscopes and techniques to enhance the contrast in tissue and view small structures. The ongoing development of new tools and technologies to study neuronal functions is essential. It is already more than a century ago, that neurons were first described as single units by Santiago Ramon y Cajal, using Camillo Golgi's approach for staining single neurons in the brain. These neurons were identified as distinct and individual units forming specialized connections to other neurons and thus building large networks of processing units. It is estimated that a single neuron can form up to 1,000 efferent synaptic connections, while receiving input from up to 10,000 afferent synaptic connections (Kandel et al., 2000). In order to understand such complicated processes as synaptic transmission, basic knowledge about the underlying cellular and synaptic mechanisms is essential. And in fact, since the early days of Camillo Golgi and Santiago Ramon y Cajal, the last century has led to a comprehensive 
understanding of such processes and how received signals are integrated (as can be inferred by the current number of published articles).

The most common signal in the brain is mediated through the neurotransmitter glutamate and the corresponding glutamate receptors such as ionotropic N-methyl-D-aspartate receptors (NMDA receptor) and non-NMDA receptors (AMPA receptors ( $\alpha$-amino-3-hydroxy-5-methyl-4isoxazolepropionic acid receptors) and Kainate receptors)), which respond to ligand binding by a conformational change, leading to the formation of an ion conducting pore. These receptors are the most important for mammalian neural systems and usually cluster at synapses by binding to intracellular scaffolding proteins, connecting them to the cytoskeleton. Furthermore, there are metabotropic glutamate receptors, which transmit their activation through G-protein mediated signaling. This might also directly or by further downstream second messengers, like calcium ions $\left(\mathrm{Ca}^{2+}\right)$, influence the activity of ion channels, through which action potentials are propagated.

In the resting state a neuron maintains a gradient of ions over its membrane. Overall this leads to an imbalance of positive and negative charges between the intracellular and the extracellular environment, building up a resting membrane potential, which is normally around $-70 \mathrm{mV}$. The binding of neurotransmitters (e.g. glutamate) to their native receptors, either directly or indirectly, leads to the formation of ion conducting pores, allowing for the flow of ions along their concentration gradients, most often, the influx of sodium ions $\left(\mathrm{Na}^{+}\right)$. This influx drives the membrane potential to a more positive potential. If a specific threshold (usually around $-55 \mathrm{mV}$ ) is reached, voltage-gated sodium channels begin to open, further accelerating the $\mathrm{Na}^{+}$-influx, starting an action potential. Through a sequence of $\mathrm{Na}^{+}$, potassium $\left(\mathrm{K}^{+}\right)$and $\mathrm{Ca}^{2+}$ channel opening and closing, these potentials are propagated along the axon to presynaptic terminals, where the action potential triggers $\mathrm{Ca}^{2+}$-influx through voltage gated calcium channels (VGCCs). This influx of $\mathrm{Ca}^{2+}$ mediates the controlled exocytosis of vesicles resting in the pre-synapse, releasing their cargo into the synaptic cleft, where the released neurotransmitter in turn binds to appropriate receptors on the postsynaptic site. This is a very simple description of the basic principle of neuronal signal propagation.

Given the complexity of signals that are perceived and processed by the brain, this mechanism can of course not be that simple. The brain has the remarkable ability to adapt in response to experience and variations in the environment, therefore synapses do not remain stable and new connections are continuously formed as well as lost. The release of neurotransmitters to a post-synapse does not always provoke the same effect with the very same amplitude, because synapses change their sensitivity to neurotransmitter over time and due to their history of previous stimulations. Such changes are in short referred to as neuronal or synaptic plasticity.

Incoming signals are not always processed the same way, since this depends on the type and amount of received transmitter, the available postsynaptic receptors and the downstream molecular mechanisms, specific for this postsynaptic. Incoming signals are even treated differently depending on location and temporal patterns and the same neurotransmitter might have opposite effects on 
different neurons and even on the same neurons at different time points of development. Though well accepted nowadays, not many scientists believed in the idea of a brain that can change in neurochemistry and anatomy according to environmental stimuli, until the 1960s (Rosenzweig, 1996). Today we know that such mechanisms are essential for brain function and play an important role in recovery after brain damage (Pascual-Leone et al., 2005). Molecular mechanisms that translate patterns of activity into specific synaptic changes, subsequently influencing synaptic transmission e.g. the number and strength of synapses can be changed by neuronal activity (Bliss and Collingridge, 1993; Kandel, 2001; Linden, 1994; Malenka and Nicoll, 1999; McEwen, 1999). Synaptic changes that lead to an enhanced sensitivity contribute to processes termed short- and long-term potentiation (STP/LTP). Events causing decreased sensitivity of synapses contribute to a process termed short- or long-term depression (STD/LTD) (Kandel et al., 2000). Such changes could result from the integration of more or less receptors into the synaptic membrane, the modification of already available receptors and channels in order to make them more or less sensitive and active, or the synthesis and release of proteins that regulate synaptic strength. Even though significant effort has already been put into exploring the underlying mechanisms of synaptic plasticity and much is already known, the involvement of many proposed proteins still remains unclear. The understanding of such mechanisms critically depends on the understanding of the detailed molecular functions of involved molecules. During the last decades, specifically one group of molecules was considered to serve as a mediator of synaptic plasticity, the group of neurotrophins. Their expression and localization has been found to change in response to neuronal activity and in turn, these molecules were shown to modulate synaptic transmission, contribute to nerve growth, the formation of new synapses, or modify properties at the individual synapse, like synapse size and protein composition (Berninger and Poo, 1996; Levi-Montalcini, 1987).

Neurotrophins are small, secreted proteins that have been found to play key functional roles in the development, neuronal homeostasis and plasticity of the peripheral and the central nervous system (PNS/CNS) in vertebrates (Bibel and Barde, 2000; Lewin and Barde, 1996; Snider, 1994). Surprisingly this group of molecules consists of only a handful of members. The most important ones are nerve growth factor (NGF), brain-derived neurotrophic factor (BDNF), neurotrophin-3 (NT-3) and neurotrophin-4 (NT-4) (Binder and Scharfman, 2004). Of these, particular interest has been focused on BDNF, because of its strong and direct action as a modulator and mediator of synaptic plasticity.

For this dissertation, we focus on the functions of BDNF in the hippocampus of the rat. The hippocampus was chosen because it is a very well-studied model structure that consists of wellknown cell types and it has a highly important function in the translation of short-term memory into long-term memory (Milner et al., 1998). We use rats as a model system, because: 1 . human tissue was not available, 2. they have been used already for substantial groundwork, and 3. They have been shown to be essentially similar to humans in many underlying molecular mechanisms (Clark and Squire, 2013). 


\section{Brain-derived neurotrophic factor (BDNF)}

BDNF was initially discovered and described for its function in mediating neuronal cell survival (Hofer and Barde, 1988; Leibrock et al., 1989), outgrowth of neuronal processes (Lindsay, 1988; McAllister et al., 1995) and neuronal differentiation during brain development of both the peripheral and central nervous system (Huang and Reichardt, 2001).

However, recent research efforts have focused more on its role in modulating synaptic plasticity. Its structure and function is highly conserved throughout vertebrates, underlining its importance. Most research has been done in rats, mice or derived cultures. During development of the CNS of the rat embryo, BDNF expression is low compared to the other neurotrophins (NT-3 and NGF) and first significant expression can be detected around day 11-12 of embryological development with expression reaching higher levels by day $12-13$, coinciding with the onset of neurogenesis (Maisonpierre et al., 1990). Despite the fact that early purifications estimated that there is only around $5 \mathrm{ng} / \mathrm{g}$ of BDNF found in the adult pig brain (Barde, 1988; Barde et al., 1982), its mRNA and protein can be found in many areas of the adult rat e.g. in large regions of the cortex, the basal ganglia and hippocampus (Conner et al., 1997; Ernfors et al., 1990) - areas important for cognition, learning and emotion, but also voluntary motor control. In experiments where BDNF was removed by the creation of homozygous knockout mice, animals developed at reduced sizes. In mouse strains with poor caretaking of nursing females such newborns die within the first 2 postnatal weeks, otherwise they normally live for up to 1 month, but exhibit severe deficiencies in coordination and balance associated with excessive degeneration in several sensory ganglia, including the vestibular ganglion, resulting in phases of spinning during periods of hyperactivity alternating with ataxia and recurrent episodes of tonic clonic seizures. Even though heterozygous animals were not reported to possess any overt behavioral or developmental abnormalities (Ernfors et al., 1995; Ernfors et al., 1994; Pozzo-Miller et al., 1999), early- and late-LTP recorded in slices was affected in both homozygote and heterozygote groups of knockout animals while basal transmission was unaffected. The effect can be rescued by exogenous application or virus mediated overexpression of BDNF, and induced in slices from wild-type animals by the application of BDNF scavenging molecules (Korte et al., 1998; Patterson et al., 1996). Conversely exercise is reported to increase physiological levels of BDNF and has been implicated in exercise-mediated cognitive improvements (Szuhany et al., 2015). However, because of the very complex role of neurotrophins, which is highly tissue specific, we will mainly focus on the function of BDNF in the CNS, or more precisely the hippocampus, because of its importance for learning and memory.

As all neurotrophins, BDNF is produced as a high molecular weight precursor (ProBDNF) of $32 \mathrm{kDa}$, a polypeptide of 229 amino acids (aa), which is proteolytically cleaved during sorting to its mature form (mBDNF) of $14 \mathrm{kDa}$ or 119 aa (Mowla et al., 2001). But, one is easily deceived by its apparent simplicity, because the process of transcription, sorting, release and receptor binding is complex; for instance, BDNF gene expression in rats is controlled by four activity-dependent and tissue specific promotors (Aid et al., 2007) and expression can be mediated through cAMP 
responsive-element binding protein (CREB) (Shieh et al., 1998; Tabuchi et al., 2002; Tao et al., 1998), upstream stimulatory factors $1 / 2$ (USF 1/2) (Chen et al., 2003b; Tabuchi et al., 2002), and calciumresponsive transcription factor (CaRF) (Tao et al., 2002) in response to activity-related increases of the calcium concentration. Expression was also demonstrated to be influenced by mechanisms affecting chromatin structure, like DNA methylation or histone deacetylation through the recruitment of global repressors such as the zinc finger transcription factor REST/NRSF (Palm et al., 1998) and MeCP2 (Chen et al., 2003a). Methylation of cytosine residues and histone deacetylation decrease BDNF transcription in rat C6 glioma cells (Aid et al., 2007). In addition to transcriptional regulation, the BDNF gene consists of eight known 5' untranslated exons and one, 3' protein coding exon. Transcripts consist of one of the $5^{\prime}$ non-coding exons spliced to the $3^{\prime}$ coding-exon or only the coding exon. Furthermore, exon 2 contains two additional alternative splice sequences increasing the pool to a total of 11 different transcripts, all coding for the same protein. Furthermore, during posttranscriptional processing, two alternative polyadenylation sites can be used, leading to distinct populations of mRNAs with a shorter or longer 3' UTR, again doubling the available pool and exon 1 transcripts possess an additional in-frame start codon, which can serve as an alternative site for the initiation of translation extending the precursor $\mathrm{N}$-terminally by eight aa, without yet known consequences (Aid et al., 2007). Similar processes extending the available pool of human BDNF transcripts and protein have also been reported (Liu et al., 2005). Transcription is regulated and depends strongly on tissue type, physiological conditions and developmental stage. All transcripts can however be found to be expressed in the adult rat hippocampus with varying transcription levels, based on age and neuronal activity. Most transcripts have peak expression levels 3 - 6 hours after seizures are induced in rats by the injection of kainic acid (Aid et al., 2007). The evolutionary necessity of so many different transcripts strongly suggests differential sorting and transport of the different BDNF mRNAs, in combination with regulated and local translation. However, the function and localization of the individual transcripts has not yet been clarified. Even before it was known that there are so many different BDNF mRNA transcripts, BDNF mRNA and protein recruitment to dendritic sites was reported in response to $\mathrm{KCl}$-mediated membrane depolarization (Tongiorgi et al., 1997) and during recent years there has been more and more evidence for transcript specific sorting (Baj et al., 2011), e.g. from the available transcripts a group of long 3' UTR mRNA transcripts has been descripted to be present in the soma and sorted to dendrites, while another group of short 3' UTR mRNAs is confined to the soma (An et al., 2008); if the long 3' UTR mRNA was truncated dendritic targeting was impaired. In the light of such complex regulation it does not appear surprising that there has been ongoing debate about the role and function of the BDNF protein.

Endogenous BDNF was reported to be predominantly localized to somatodendritic domains in rat hippocampal neurons, where it is enriched and released in response to $\mathrm{KCl}$-mediated membrane depolarization (Edelmann et al., 2015; Goodman et al., 1996; Kuczewski et al., 2008; Tongiorgi et al., 1997). But BDNF is also anterogradly transported and released from axonal terminals (Conner et al., 1997; Dieni et al., 2012), Both cases reportedly exclude the other possibility. 
Therefore, the site of release is still heavily disputed. Other experiments rely on the overexpression of fluorescently labeled BDNF for the visualization of BDNF localization and trafficking. Such studies were focused on BDNF containing only the coding exon transcript together with a $3^{\prime}$ attached sequence coding for the fluorescent tag. BDNF-GFP was found to behave similarly to the endogenous variant and was sorted to postsynaptic sites and released in response to neuronal activity, which depended on postsynaptic ionotropic glutamate receptor activation and calcium concentration increase (Hartmann et al., 2001; Kojima et al., 2001). BDNF-GFP was also found to move to axonal terminals at which it is also apparently released (Kohara et al., 2001). Other studies reported distinct BDNF populations perambulating neuronal processes, but either exclusively axonal or dendritic (Dean et al., 2012), however without differentiating between expressed and potential endocytosed BDNF-GFP. Injected radio-labeled BDNF was found to be retrogradly transported in the rat hippocampus (DiStefano et al., 1992) and suggests that BDNF acts on presynaptic terminals, from where it is taken up and retrogradly transported. Despite the large amount of data that is available, the question of axonal versus dendritic targeting remains unclear.

Besides the differential sorting and release of BDNF, another source of debate is the processing and form of release. Under physiological conditions BDNF exists as a tightly associated, but non-covalent homodimer (Radziejewski et al., 1992) and it has been reported that it can also form heterodimers with NT-3, thus affecting the sorting of NT-3 (Farhadi et al., 2000; Heymach and Shooter, 1995; Robinson et al., 1995); though the functional purpose of such heterodimers remains elusive. The secretion of neurotrophins follows two pathways, the constitutive pathway, where neurotrophins are continuously secreted from the cell, independent of neuronal activity or other stimuli, and the regulated secretory pathway, were proteins are sorted into secretory vesicles that are released upon stimulation. In both cases proteins go through the trans-Golgi network (TGN) where they are packed in vesicles and secreted through exocytosis. There is data suggesting, that BDNF is released in its precursor form (Chao and Bothwell, 2002; Yang et al., 2009) and processing occurs extracellularly through cleavage by the serine protease plasmin or selective metalloproteases. Though some of these results were obtained from experiments using virus-mediated expression of ProBDNF in non-neuronal cultures (Heymach and Shooter, 1995; Lee et al., 2001), release of ProBDNF was also detected in experiments with mouse hippocampal cultures (Mowla et al., 2001; Pang et al., 2004) or knock-in mice, expressing ProBDNF from the endogenous promoter (Yang et al., 2014; Yang et al., 2009). A functional relevance of ProBDNF was shown, but by applying recombinant BDNF resistant to protease cleavage (Woo et al., 2005). However, the release of endogenous ProBDNF is still heavily disputed (Dieni et al., 2012; Matsumoto et al., 2008). Substantial data exists suggesting that the precursor is only released in small amounts through the constitutive pathway. The strongest indication of ProBDNF release was published by Yang et al. 2009, however their modification of the gene locus by producing BDNF-HA knock-in mice influences the transcriptional regulation and sorting of BDNF because it extends the 3' UTR (An et al., 2008; Yang et al., 2009), which might support local dendritic translation without prior processing. However a potential release 
of ProBDNF at synapses in response to activity would be interesting in terms of a plasticity based on coincidence detection (Lu, 2003), which could occur given that the extracellular conversion of ProBDNF would depend on the activity-dependent release of the tissue Plasminogen activator (tPA) from dense core granules (Lochner et al., 2006; Pang et al., 2004).

ProBDNF is sorted to the secretory pathway by binding the sorting receptor sortilin (Chen et al., 2005) and/or carboxypeptidase $E$, which also processes it at a dibasic cleavage site in the middle of the precursor protein (Lou et al., 2005; Matsumoto et al., 2008; Mowla et al., 1999). Subsequently mature BDNF (mBDNF) can be released by secretory granules (dense core vesicles) at perisynaptic sites in response to neuronal activity (Farhadi et al., 2000; Goodman et al., 1996). Overexpression of fluorescently labeled BDNF is also reported to be sorted into the secretory pathway (Haubensak et al., 1998). Interestingly the Val66Met polymorphism, a single nucleotide polymorphism in the BDNF gene that affects brain anatomy, memory and anxiety (Chen et al., 2006; Egan et al., 2003), appears to affect cell sorting and presumably also processing, decreasing the amount of releasable mBDNF and increasing constitutive release of ProBDNF (Chen et al., 2004).

There is not only uncertainty about the release site, form and regulation of BDNF, but also about the effect through which BDNF modulates synaptic transmission. Because of its highly basic properties BDNF is considered to act locally following release and synapse specifically or affect only synapses in the vicinity of the release site (Poo, 2001). BDNF was found to selectively potentiate immature synapses with low release probabilities and weak transmission, while leaving strong synapses unaltered (Berninger et al., 1999). Effects on glutamatergic transmission have been shown to be mediated by BDNF-regulated phosphorylation of synapsins, increasing the number of readilyreleasable vesicles (Gottschalk et al., 1998; Jovanovic et al., 2000; Pozzo-Miller et al., 1999; Shinoda et al., 2014). But BDNF has also been reported to act exclusively postsynaptically by affecting $\mathrm{Ca}^{2+}$ channels and NMDA receptors (Kovalchuk et al., 2002; Levine et al., 1998). Conversely, BDNF reduces transmission at inhibitory synapses (Frerking et al., 1998; Tanaka et al., 1997), while selectively increasing synaptic transmission only at excitatory synapses (Shinoda et al., 2014). Recent studies propose that BDNF acts in an autocrine or paracrine fashion. An autocrine function is implicated by the co-presence of BDNF and receptor mRNA (Miranda et al., 1993; Wang et al., 2015) and dendritic release causing dendritic effects (Edelmann et al., 2015; Wang et al., 2015). In any case, BDNF and its target receptors are highly regulated. While the mature form of BDNF is involved in cell survival and the strengthening of synaptic transmission, ProBDNF mediates LTD and apoptosis and its function primarily on activated synapses suggests some sort of underlying coincidence detection (Lu, 2003; Poo, 2001).

The biological function of BDNF is mediated by binding to two different receptors. ProBDNF binds to the pan-neurotrophin receptor p75NTR (also LNGFR for low-affinity nerve growth factor receptor, or NGF receptor - a member of the NGF/TNF receptor superfamily), and mature BDNF binds to the tropomyosin receptor kinase $B$ (TrkB receptor). All neurotrophins bind non-selectively to p75NTR, as well as to their specific receptors, TrkA (in the case of NGF), TrkB (for BDNF), and TrkC 
(for NT-3) (Chao and Bothwell, 2002; Kaplan et al., 1991). Activation of p75NTR by neurotrophic precursors mediates cell apoptosis (Lee et al., 2001) and selective binding of ProBDNF has been implicated in NMDA receptor mediated LTD (Woo et al., 2005). In accordance with this, deletion of the p75NTR gene results in increased spine density and complexity (Zagrebelsky et al., 2005). Even though the existence of the p75NTR and its functions are already known for a long time, its signaling is not as well understood as that of TrkB, but it has been reported to involve JNK, p53, and NF-KB signaling (Bibel and Barde, 2000; Chao and Bothwell, 2002; Kaplan and Miller, 2000).

When processed and released, mBDNF binds with high affinity to and activates the TrkB receptor (Soppet et al., 1991; Squinto et al., 1991). mBDNF binding to TrkB mediates conformational changes in the receptor ( $\mathrm{Li}$ et al., 2002) resulting in dimerization and autophosphorylation of the intracellular tyrosine residues, consequently activating the tyrosine kinases. Activated tyrosine kinases are involved in triggering many different downstream signaling pathways including the MAPK (mitogen-activated protein kinase) pathway, the phosphatidylinositol 3-kinase (PI3K) pathway, and the phospholipase C- $\gamma$ (PLC- $\gamma$ ) pathway (Kaplan and Miller, 2000). These signals commonly participate in the regulation of gene expression. It is also speculated that TrkB signaling induces local synthesis of proteins associated with spine growth and plasticity at stimulated spines. Locally synthesized proteins promote actin dynamics and AMPA receptor trafficking in spines, which leads to growth of the spine head and formation of stable LTP (An et al., 2008). Besides the full-length version, the TrkB receptor gene locus produces 2 known truncated variants of this receptor (TrkB.T1 and TrkB.T2) missing the intracellular tyrosine kinase domains (Klein et al., 1990). While full-length TrkB and T1 are expressed in neurons, differentiated astrocytes in the hippocampus are known to only express the truncated T1 form (Climent et al., 2000; Colombo et al., 2012; Rose et al., 2003; Rudge et al., 1994). Initially the truncated versions were thought to function primarily as scavengers through rapid internalization upon binding, limiting BDNF diffusion (Biffo et al., 1995; Fryer et al., 1996). However they have been reported to transmit BDNF binding by non-canonical downstream signal pathways, that are not yet well defined (Aroeira et al., 2015; Baxter et al., 1997), e.g. truncated, but not the full-length form are reported to be expressed in astrocytes mediating BDNF binding and causing calcium influx, presumably through activation of the PLC pathway or to influence astrocyte morphology through the non-canonical (RhoGDI1) signaling pathways (Aroeira et al., 2015; Ohira et al., 2007; Ohira et al., 2005; Rose et al., 2003).

Both BDNF and TrkB receptor mRNA is highly abundant in the rodent hippocampus (Miranda et al., 1993) as is TrkB immunoreactivity in rats (Yan et al., 1997). But other than the TrkB receptor variants (full-length and 2 known truncated isoforms) the p75NTR is reported to be strongly expressed in the developing rat cortex, but only weakly in the adult hippocampus (Yan and Johnson, 1988, 1989; Yan et al., 1997). While rat astrocytes exhibit strong expression levels of p75NTR in the glia limitans, lining the hypothalamus and amygdala, p75NTR expression is only weakly detected in hippocampal cultures of neurons and astrocytes (Rudge et al., 1994). This is interesting because there is a significant history of LTD studies based on the presumptive binding of ProBDNF to the 
p75NTR in hippocampal cultures of mice (Woo et al., 2005; Yang et al., 2014; Yang et al., 2009). Even though from these studies it is also apparent that p75NTR expression levels are high during development and early adulthood, but subsequently decline to much weaker levels. Therefore, it can be generally assumed that P75NTR is present in the hippocampus of the rat and mouse during adulthood, though at comparably weaker levels than TrkB. Similar to BDNF knockout mice, homozygous TrkB knockout animals do not display feeding activity, and mostly die within the first postnatal week. Neuroanatomical examination of TrkB knockout mice revealed neuronal deficiencies in the central (facial motor nucleus and spinal cord) and peripheral (trigeminal and dorsal root ganglia) nervous systems (Klein et al., 1993). Surface expression of the TrkB receptor is also regulated in response to neuronal activity, e.g. TrkB is reported to be inserted into the membrane in response to tetanic electrical stimulation, which depends on calcium influx (Du et al., 2000), further supporting the concept of coincidence detection.

Given all of these interesting implications it is very likely that BDNF can indeed act through both constitutive and regulated release from pre- and postsynaptic sites affecting molecular mechanisms on both sites of the synaptic cleft (Edelmann et al., 2014). Though some important questions remain, for instance regarding the function of BDNF release in response to activity. For example: Are DCVs recruited to synapses upon stimulation? What happens to the released BDNF when it is bound to TrkB and its truncated receptors? Is it endocytosed and degraded or eventually used for re-release, as suggested by rescue experiments in which recombinant BDNF is applied to cultures (Korte et al., 1998; Patterson et al., 1996; Santi et al., 2006)? Does the binding of BDNF have functional consequences for non-neuronal cells?

\section{BDNF signaling dysregulation is involved in certain pathologies and disorders}

Due to the complex regulation of BDNF and its importance as a mediator for synaptic plasticity, it is not surprising that small changes in BDNF can have strong functional consequences. Changes in the regulation of BDNF have been implicated in a number of disorders: In humans, a prominent polymorphism (Val66Met) exists in an estimated 30-50\% of people worldwide that are either homozygous or heterozygous for this single nucleotide polymorphism (SNP) leading to the exchange of valine at the $66^{\text {th }}$ position of BDNF for methionine (Hartmann et al., 2012). The mutation occurs in the precursor region and BDNF with the Met polymorphism fails to be sorted to the regulated secretory pathway, with the functional consequence of decreased episodic memory in patients (Chen et al., 2004; Egan et al., 2003). In Huntington's disease release of BDNF through the regulated secretory pathway also is decreased (Hartmann et al., 2012). Reduced levels of hippocampal BDNF mRNA and protein have been reported in Alzheimer's disease and are suspected to contribute to progression of the disease (Connor et al., 1997; Phillips et al., 1991) and decreased BDNF protein levels have also been demonstrated in the substantia nigra in Parkinson's disease (Binder and Scharfman, 2004). On the other hand, elevated BDNF levels are associated with increased pain sensation for inflammatory injury conditions in peripheral nerves and can lead to neuropathic pain in 
the CNS, in which normal touch and pressure somatosensation becomes painful (Binder and Scharfman, 2004). BDNF has further been implicated in a number of psychological disorders like depression,' where BDNF mRNA levels are decreased; on the contrary physical exercise increases BDNF mRNA and alleviates depressive symptoms. Lithium, a major drug for the treatment of bipolar disorder, has also been shown to increase BDNF-TrkB signaling pathways in cerebral cortical neurons (Binder and Scharfman, 2004). The detailed understanding of the regulatory functions and players in these BDNF-mediated pathways might help to further understand the cause of certain disorders and pathologies and help in finding treatments. An investigation of the possible recruitment of BDNF to specific sites in response to focal synaptic activity is important to understand how BDNF functions in response to normal and pathological increases in neuronal activity; this relies on techniques for the manipulation of focal synaptic activity.

\section{Techniques to probe synaptic plasticity}

Approaches to determine the biological mechanisms underlying synaptic plasticity focus on the artificial stimulation of cells. Changes in cellular chemistry, protein localization and signal propagation properties can subsequently be investigated. To achieve this a large set of diverse techniques has been developed, dedicated to stimulating neurons and probing their responses. Commonly used approaches include whole-cell depolarization, usually aimed at the activation of voltage-gated channels, consequentially leading to action potentials or an increase of the intracellular $\mathrm{Ca}^{2+}$-concentration. The easiest way to depolarize neurons is chemically, either by causing membrane depolarization with increased extracellular $\mathrm{KCl}$, electric field stimulation or by application of neurotransmitters, e.g. glycine and glutamate. $\mathrm{KCl}$-mediated membrane depolarization experiments are performed at 10 to $50 \mathrm{mM}$ extracellular $\mathrm{KCl}$ over the time course of a few hours and up to a few days and have yielded significant physiological data without obvious reports of cytotoxicity (Tongiorgi et al., 1997; Zafra et al., 1990). However higher $\mathrm{KCl}$ concentrations will keep cells at high membrane potentials, and may not allow voltage-gated ion channels to de-inactivate, which might mitigate prolonged neuronal stimulation. Pharmacological agents are also used to increase neuronal network activity, e.g. forskolin (stimulating adenylyl cyclase and raising intracellular cAMP levels) or bicuculline (an antagonist for the $\mathrm{GABA}_{\mathrm{A}}$ receptor, which decreases the effect of inhibitory synaptic conections in the neuronal network). Glutamate and $\mathrm{KCl}$ can also be applied locally, using micropipettes. This already allows for the investigation of many important signaling parameters, but generally does not allow the study of molecular mechanisms underlying synaptic plasticity at single synapses, since the diffusion of molecules will always affect nearby structures. Quite a few promising approaches have been undertaken in the past, allowing activation of areas in the range of several $\mu \mathrm{m}$ and even small subcellular structures such as single spines. Photouncaging of extracellular glutamate, acetylcholine (ACh), gamma aminobutyric acid (GABA) or even intracellular $\mathrm{Ca}^{2+}$ can be used to further increase the precision of stimulation by 'releasing' transmitter and second messenger in small doses and close to their target structures (Adams and 
Tsien, 1993; Bartels et al., 1971; Pettit et al., 1997; Wang and Augustine, 1995). Photo-uncaging uses molecules covalently bound to a photo-sensitive blocking group 'cage', making them biologically inactive, because they are not able to interact with their native binding partners. As such they can be applied to the extracellular medium/solution or in the case of caged $\mathrm{Ca}^{2+}$, loaded into the cell with no effect on their target structures. When the caged molecules are exposed to brief UV light pulses, the covalent bond is broken and the molecule is released, regaining its biological function. This has several advantages over local application of neurotransmitters or $\mathrm{KCl}$. The resolution that can be achieved using UV-light exposure is only limited by the diffraction-limit of light and can be restricted to subcellular structures on a cell within the field of view, significantly decreasing the depolarized area (O'Neill et al., 1990; Wang and Augustine, 1995). However, out-of-focus light above and below the focal plane limits the resolution of the stimulation, even when focused to diffraction-limited spot sizes below $1 \mu \mathrm{m}$. Double-caged molecules and two-photon uncaging significantly increase the resolution because two photons have to hit the caged molecule at the same time. Similar to the twophoton imaging technique this occurs with higher probabilities in the focal plane, where the photon density is the highest, further decreasing the volume in which molecules will be released (Adams and Tsien, 1993; Kantevari et al., 2010; Pettit et al., 1997). These techniques have been optimized to even allow for the use of optically independent two-photon uncaging of glutamate and GABA (Kantevari et al., 2010). Using photo-uncaging, small volumes of neurotransmitter can be directly released at the desired region. The obvious main advantage of this technique is that the stimulus to the postsynaptic cell is presumably close to physiological. But its advantage is also a disadvantage because it only stimulates postsynaptic receptors and does not allow for the stimulation/depolarization of native presynaptic sites. Caged $\mathrm{Ca}^{2+}$, however, would allow for the stimulation of $\mathrm{Ca}^{2+}$-gated ion channels at presynaptic sites, but is yet not available for two-photon-uncaging, and spatiotemporal precision at the scale of single release events or spikes cannot be achieved using caged compounds. Moreover, diffusion of released molecules, even though for small volumes, cannot be controlled and the double-caged approach limits release events, since every light pulse partially releases molecules, the concentration of these molecules will increase over time and at some point, will become fully activated by a second light pulse, even outside of the plane of focus.

Other techniques such as using two electrodes for direct current field stimulation allow stimulating all cell structures, but cannot easily be focused to a specific small area. A way to combine focal stimulation with current injection is by using micropipettes to directly inject stimulating currents to the cell. Single cells and even subcellular parts of cells can easily be stimulated using this technique. But focal activation of single synapses is unlikely to be achieved precisely, because it is an invasive technique; in order to leave synapses intact, they cannot directly be patched and therefore the strength and field of current required to stimulate a single synapse would also affect neighboring regions. More recently, new noninvasive approaches were developed using light to change the activity of neurons. The effect of fluorophore/chromophore-assisted light inactivation can be used to affect the function of nearby proteins and can, for instance, be used to inhibit release from individual 
presynaptic sites (Lin et al., 2013). Furthermore, the use of light was expanded to include modified proteins. These approaches include: 1. genetically engineered photosensitive G-proteins (with light mediating activation of cation-channels via phospholipase $\mathrm{C}$ ), 2. artificially designed chemical gates controlling $\mathrm{K}^{+}$-channel gating (a molecule which binds to a genetically engineered $\mathrm{K}^{+}$-channel, a corresponding pore blocker and a photo-isomerizable azobenzene, promoting conformational changes due to light mediated cis- and trans-state transitions; When exposed to light the blocker is retracted allowing conductance) and 3. chemically modified ionotropic receptors (ionotropic purinoceptors and capsaicin receptors modified with photo-removable blocking groups) (Banghart et al., 2004; Lima and Miesenbock, 2005; Zemelman et al., 2002). However, such approaches currently only offer temporal control on the order of seconds to minutes and was superseded by the rise of another technique, the use of algal photoreceptors (Hegemann, 2008).

There are many different kinds of photosensitive proteins that have evolved in algae, like photoreceptive enzymes and ion-pumps, but also light-gated cation-channels. The expression of these so-called channelrhodopsins (ChRs), natively obtained from the algal Clamydomonas reinardhtii allowed for the first time light-gated millisecond-timescale optical control of membrane depolarization (Boyden et al., 2005; Li et al., 2005; Nagel et al., 2003). Channelrhodopsins are sensory photoreceptors that promote phototaxis in algae in order to optimize the light conditions for photosynthetic growth. Initially two native sub-types were described: ChR1, a proton-conducting channel and ChR2, a nonselective cation-channel. The photocurrents elicited by illumination of ChR1 were not strong enough to cause sufficient depolarization (Nagel et al., 2005b). When expressed in neurons and exposed to blue-light, ChR2 opens and allows for the conductance of ions along their concentration gradient for the duration of light exposure. The ion flux leads to electrophysiologically measurable photocurrents of several hundred pA, depolarizing the cell and thus driving it to fire action potentials. Since its discovery, ChR2 was successfully established as one of the most often used tools in the field of neuroscience and is commonly applied to a huge number of diverse model systems, both in vitro and in vivo (Deisseroth, 2011). ChR2 channels were mutated for mammalian expression and are continuously undergoing optimization to generate a number of different variants with different properties. For instance, the H134R mutant promotes enhanced stationary photocurrents (Nagel et al., 2005a), E123T variants exhibit accelerated photocycles (Gunaydin et al., 2010), C128S, C128T and C128A mutants exhibit prolonged open channel states (Berndt et al., 2009), L132C variants are calcium conducting (Kleinlogel et al., 2011) and many more are know by now. The photocycle of these channels has been intensively studied (Figure 1) (Bamann et al., 2010; Bamann et al., 2008; Nikolic et al., 2009; Stehfest et al., 2010). When the channel is illuminated in its darkadapted closed state (D480), it undergoes a conformational change to the open channel state (P520), subsequently it passes through several intermediate states, until it finally reaches its dark-adapted closed state again and the cycle can be repeated. Interestingly it has been found that some intermediates, P390 and the open state P520 are sensitive to either UV-light ( $390 \mathrm{~nm}$ ) or a more red-shifted ( $520 \mathrm{~nm}$ ) wavelength, which will mediate a transition to the closed channel-state (P480), 
as depicted by the arrows in Figure 1b. Due to its fast gating kinetics, this is usually not of practical use. The ChR2 C128S, C128T and C128A mutants, termed step-function-opsins and related channels, possess long openchannel states where photocurrents can be efficiently stopped with the exposure of a second closing wavelength. Especially these variants exhibit another distinctive feature; at the late stage of their photocycle, they can branch-off into a long-lasting, non-conducting state (P380). Channels trapped in this state will take a few minutes to enter their dark-adapted (D480) ground state again and during this time cannot contribute to the photocurrent. This state is supposedly entered due to prolonged stimulation, but can be significantly reduced, if channels are closed prematurely by illumination with the respective closing wavelength (Schoenenberger et al., 2009). Further ChR2related ion-channels and ion-pumps have been found in different organisms, extending the spectrum of available channels with different properties even further. Detailed information about channel structure and function is

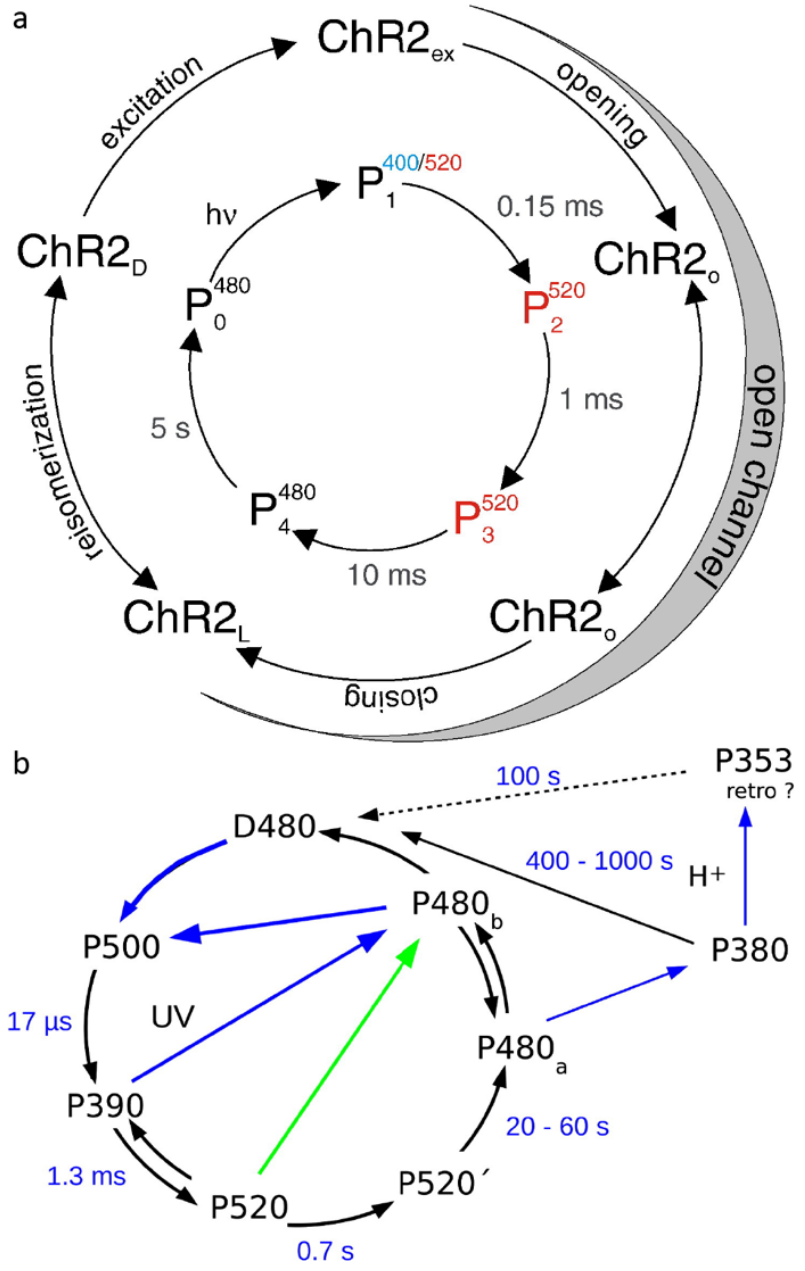

Figure 1: Proposed basic ChR2 photocycle (a) proposed by and graphic borrowed from Bamann et. al 2008 (respective figure 5) and a further extended variant based on the $\mathrm{C} 128$ variants (b) proposed by and graphic borrowed from Stehfest et al. 2010 (respective figure 8).

expected to lead to more sophisticated modifications and channel variants in the future.

So far the stimulation of neurons using channelrhodopsin has been mainly focusing on targeting specific cell populations or areas in the brain of rodents (Gradinaru et al., 2010; Gunaydin et al., 2010), but ChRs can also be used to stimulate subcellular areas. Several attempts have been made to decrease the area of activation by optimization of focal illumination and by making use of two-photon illumination, decreasing out-of-focus activation (Mohanty et al., 2008; Packer et al., 2012; Schoenenberger et al., 2008). A very elegant approach for the physiological and controlled stimulation of postsynaptic sites can be achieved by optogenetic stimulation of the presynaptic neuron (Zhang and Oertner, 2007). Synapses can then be identified by colocalization, e.g. of a fluorescently labeled PSD, expressed in the postsynaptic neuron with the fluorescent signal from the ChR in the presynaptic neuron. These approaches have been combined with electrophysiological stimulation of the postsynaptic neuron, demonstrating the usefulness of this technique for the study of synaptic plasticity (Zhang and Oertner, 2007). Recent combinatorial expression of spectrally 
separable light-gated channels with $\mathrm{pH}$-sensitive fluorophores and genetically encoded calcium indicators provides an all-optical approach for multiplex control and tracking of neuronal activity (Li and Tsien, 2012).

Several groups already focused on the use of hChR2 in order to control neural activity and achieve focal stimulation and using focused laser beams with reduced laser power spatial selectivity of $<30 \mu \mathrm{m}$ was achieved (Schoenenberger et al., 2008). Two-photon stimulation was already tested by using $920 \mathrm{~nm}$ light, achieving a diffraction limited stimulation spot of 1.1 $\mu \mathrm{m}$ (Mohanty et al., 2008; Papagiakoumou et al., 2010). However, most of these techniques aim to elicit action potentials and so far, no technique exists that allows for the specific and highly focal control of activity in both pre- and postsynaptic structures. Such methods could significantly contribute to the broadening of our understanding of detailed signaling processes in neuronal networks and the involvement of molecules in the regulation of synaptic plasticity at individual synapses or even sub-synaptic sites.

We developed a possible approach to stimulate single synapses or smaller areas by using step-function opsins, making use of their switching properties and illumination inspired by STED/ RESOLFT microscopy. A center focused light beam would be used to activate channels, while a surrounding but partially overlapping illumination with the respective closing wavelength of channels would eliminate the photocurrent in this area, thus allowing unprecedented nano-regions of focal activation down to single channels.

The goal of this dissertation was to test the feasibility of currently available channels for the stimulation of single synapses using this technique in order to study cellular processes underlying synaptic plasticity, in particular the recruitment of BDNF to synaptic sites. This idea arose from preliminary results in which neurotrophins were recruited to synaptic sites in neuronal networks stimulated with LTP-induction protocols, where LTP of synapses is thought to underlie learning and memory. Such results prompted the novel hypothesis that neurotrophins (and perhaps other signaling molecules) may be targeted specifically to activated synapses to modulate their strength, for example during the acquisition of new information during learning. In order to test this idea directly, one needs the ability to stimulate single synapses and to determine if neurotrophins are specifically directed to these activated sites. To achieve this, precise activation of individual synapses is essential. 


\section{Materials and Methods}

\subsection{Suppliers}

Unless specifically indicated, lab consumables were bought from STARLAB GmbH ('STARLAB/CytoOne'; Hamburg, Germany; www.starlab.de), Sarstedt AG \& Co. (Nümbrecht, Germany; www.sarstedt.com), Greiner Bio-One GmbH (Frickenhausen, Germany; www.gbo.com/bioscience), Eppendorf Vertrieb Deutschland GmbH (Wesseling-Berzdorf, Germany; www.eppendorf.com) or Carl Roth GmbH \& Co. KG ('Roth'; Karlsruhe, Germany; www.carlroth.com). Chemicals were also bought from Carl Roth, filters and membranes from Merck Chemicals $\mathrm{GmbH}$ ('Merck/Millipore'; Darmstadt, Germany; www.merckmillipore.com) and reagents, media, serum or restriction enzymes for molecular biological work were bought from Fisher Scientific GmbH ('Thermo Scientific/Life Technologies/Gibco/Invitrogen', Darmstadt, Germany; www.thermofisher.com). Some chemicals and DNA oligos for sequencing were ordered from Sigma-Aldrich Chemie GmbH ('Sigma'; Munich, Germany; www.sigmaaldrich.com) and sequencing services were conducted through GATC Biotech AG (Konstanz, Germany; gatc-biotech.com). Extensive subcloning and mutagenesis tasks were directed to GenScript (Piscataway, U.S.A.; www.genscript.com). In addition, kits for DNA purification and gel extraction were bought from MACHEREY-NAGEL GmbH \& Co. KG ('MN'; Düren, Germany; www.mn-net.com) and QIAGEN (Hilden, Germany; www.qiagen.com) and for transfection of mammalian cells using electroporation from Lonza Cologne GmbH(Cologne, Germany; www.lonza.com).

All microscopes used for this dissertation were produced by Carl Zeiss Microscopy $\mathrm{GmbH}$ ('Zeiss'; Göttingen, Germany), electrophysiological recordings were done using amplifiers and software from HEKA Elektronik GmbH (Lambrecht/Pfalz, Germany; www.heka.com) and micromanipulators/pipette puller were bought from Sutter Instruments Co. (Navato, U.S.A.; www.sutter.com).

\subsection{Molecular biology}

\subsubsection{Vectors}

\section{Channelrhodopsins}

Most common vectors for CaMKIl $\alpha$-controlled expression of channelrhodopsins used in this dissertation (pAAV-CaMKIla-'hChR2'-mCherry/EYFP-WPRE), were provided by the lab of Karl Deisseroth, MD, Ph.D. (Stanford University/Howard Hughes Medical Institute, U.S.A.). These include the H134R, T159C, E123T/T159C and C128S/D156A ChR2 variants (Berndt et al., 2011; Yizhar et al., 2011) and ChR1 VChR1 chimera C1V1 E122T/E162T (Erbguth et al., 2012), as well as a P2A-motif combined eNpHR3.0-EYFP (halorhodopsin) and hChR2(H134R)-mCherry fusion construct, termed eNPAC (pAAV-hsyn-eNPAC) (Gradinaru et al., 2010). 
Another ChR2 variant that has been published to be calcium permeable, L132C and therefore termed calcium translocating channelrhodopsin (CatCh) (Kleinlogel et al., 2011), has been provided by Dr. Ernst Bamberg (Max-Panck-Institute for Biophysics, Germany).

With the assistance of GenScript pAAV-CaMKIl $\alpha$-hChR2(H134R)-mCherry-WPRE, pAAVCaMKIl $\alpha$-hChR2(T159C)-mCherry-WPRE and pAAV-CaMKIla-C1V1(E122T/E162T)-TS-mCherry were mutated to combine common ChR characteristics and yield the slow-photocycle high-conducting variants hChR2(C128A/H134R/T159C), hChR2(C128A/L132C/T159C) and C1V1(E122T/C167S) in the same backbone.

Additional ChRs used for this work, selected for their strong photocurrents, Chronos (pN3Chronos) and CoChR (pN3-CoChR), together with their mutated SFO and SSFO variants, Chronos C145S and C145S/E162T and CoChR C108S and C108S/D136A, under CMV promotor control, were provided by Edward Boyden, Ph.D. (Massachusetts Institute of Technology, U.S.A.)

\section{Fluorophores and other fluorescently labeled proteins}

Vectors for the expression of EGFP under synapsin (pAAV-6P-noTB-SEWB) and GFAP (pAAV-GFAP2.2EGFP-WPRE) promoter control, as well as for the expression of LSSmOrange (pMito-LSSmOrange, Addgene \#37135) and the genetically encoded calcium indicator RCaMP1c (Akerboom et al., 2013), originally made by the group of Loren L. Looger, Ph.D. (Janelia Farm Reseach Campus/Howard Hughes Medical Institute, Ashburn, U.S.A.), were provided by Dr. Sebastian Kügler (University Medicine Göttingen, Department of Neurology, Germany). By molecular cloning the LSSmOrange fluorophore was exchanged for the two cell type specific promotors to yield pAAV-hsynLSSmOrange-WPRE and PAAV-GFAP2.2-LSSmOrange-WPRE (refer to the section about molecular cloning for further detail).

A vector for the expression of rat proBDNF, fused to mRFP1 (pBa-BDNF-mRFP1), originally made by the group of Gary Banker, Ph.D. (Oregon Health \& Science University, U.S.A.) was provided by Michael Silverman, Ph.D. (Simon Fraser University, Canada). And the vector with the corresponding receptor, TrkB (pEGFP-N1-TrkB, Addgene \#32500), originally produced in the lab of Rosalind Segal, MD, Ph.D. (Dana-Farber Center/Harvard Cancer Center, U.S.A.) was provided by Katja Burk, Ph.D. (European Neuroscience Institute Göttingen, Germany). By molecular cloning, TrkB-EGFP was put in the pAAV-hsyn-LSSmOrange backbone, to make pAAV-hsyn-TrkB-EGFP/LSSmOrangeWPRE (refer to the molecular cloning section for further detail). With the assistance of GenScript, the backbone of the proBDNF-mRFP1 was swapped with the one from pAAV-CaMKIla-hChR2(T159C)P2A-EYFP-WPRE, provided by the lab of Karl Deisseroth, MD, Ph.D.; at first, to get pAAV-CaMKIlaBDNF-mRFP1-P2A-EYFP-WPRE and subsequently exchanging the EYFP to ECFP, taken from a pNicePSD95-CFP, provided by Peter Scheiffele, Ph.D. (University of Basel, Switzerland). The start codon in front of the ECFP was removed to yield pAAV-CaMKII $\alpha$-BDNF-mRFP1-P2A-ECFP. Either a ChR2 T159C or L132C was then added in front of the second fluorophore (PAAV-CaMKII $\alpha$-BDNFmRFP1-P2A-'ChR'ECFP). ECFP was later mutated to EGFP, to yield pAAV-CaMKII $\alpha$-BDNF-mRFP1-P2A-EGFP-WPRE. 
Based on this, BDNF was removed from the construct to yield pAAV-CaMKIl $\alpha$-mRFP1-P2A-EGFPWPRE and the two fluorophores were swapped to produce pAAV-CaMKII $\alpha-B D N F-E G F P-P 2 A-m R F P 1-$ WPRE.

CMV promoter-driven expression vectors for GFP labeled EEA1 (GFP-EEA1) and RAB5A (Rab5a-eGFP), were provided by the lab of Pietro De Camilli, MD (Yale School of Medicine, U.S.A.) and RAB7A (GFP-rab7, Addgene \#12605), LAMP1 (LAMP1-mGFP, Addgene \#34831) and LC3 (pEGFP-LC3, Addgene \#24920) available from Addgene were provided by the lab of Ira Milosevic, Ph.D. (European Neuroscience Institute Göttingen, Germany).

A construct for the expression of fluorescently labeled Neurexin (pCAG-mRFP-Nrx), with the fluorophore present on the $\mathrm{N}$-terminal extracellular part of the protein, was provided by Peter Scheiffele, Ph.D. (University of Basel, Switzerland).

Vectors for the production of AAVs

Vectors used to produce AAV serotype $1 \& 2$ chimeric virions, pH21 containing serotype 1 and pRV1 containing serotype 2 replication and capsid sequences (Klugmann et al., 2005), were provided by the lab of Moritz Rossner, Ph.D. (Psychiatric Clinic of the Ludwig-Maximilians-University) and the adenovirus helper plasmid pF $\Delta 6$ was provided by Susanne Schoch McGovern, Ph.D. (University Hospital of Bonn, Germany).

\section{Adeno-associated virus (AAV)}

Purified and concentrated AAV serotype 6 virions for the delivery of hsyn-EGFP $\left(2 \times 10^{8} \mathrm{vp} / \mu \mathrm{l}\right)$, hysn-ProBDNF $\left(1.9 \times 10^{8} \mathrm{vp} / \mu \mathrm{l}\right)$, CaMKIl $\alpha$-BDNFmRFP1-P2A-EGFP $\left(0.7 \times 10^{8} \mathrm{vp} / \mu \mathrm{l}\right)$ and CaMKIl $\alpha$ mRFP1-P2A-EGFP $\left(2.5 \times 10^{8} \mathrm{vp} / \mu \mathrm{l}\right)$, the latter two for injection into organotypic brain slices, were provided by Sebastian Kügler, Ph.D. (University Medical Center Göttingen, Department of Neurology, Germany).

\subsubsection{Plasmid amplification and molecular cloning}

\section{Amplification}

Most vectors were amplified with the use of XL1-Blue E. coli cells from Stratagene (cat. no. 200249; Agilent Technologies, Santa Clara, U.S.A.). In the case plasmids were prone to recombinatorial events or deletions, e.g. very large plasmids ( $>7 \mathrm{~kb}$ ) and plasmids harboring ITRs for AAV production, vectors were amplified using SURE (Stop Unwanted Rearrangement Events) cells (Stratagene; cat. no. 200238). Cells were grown in LB-media after the formulation of Luria/Miller (Roth; cat. no. X968.1), containing the appropriate antibiotic for positive selection (either $100 \mu \mathrm{g} / \mathrm{ml}$ Ampicillin or $50 \mu \mathrm{g} / \mathrm{ml}$ Kanamycin) and were incubated at $37{ }^{\circ} \mathrm{C}$ and $250-290 \mathrm{rpm}$. LB-Agar plates additionally contained $15 \mathrm{~g} / \mathrm{l}$ Agar (Roth; cat. no. 5210.2).

Cells were made electrocompetent by following a protocol provided by Sandra Ott-Gebauer (European Neuroscience Institute Göttingen, Germany). In brief, $4 \times 500 \mathrm{ml}$ cultures were grown without antibiotics until an $\mathrm{OD}_{600}$ of around 0.6. Cells were pelleted 3 times at $8,000 \mathrm{rpm}$ for $20 \mathrm{~min}$ 


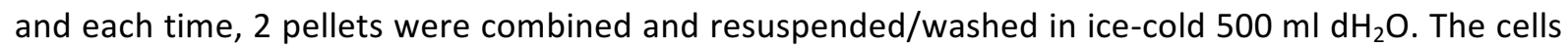
were centrifuged 3 more times and first resuspended in $400 \mathrm{ml}$, second in $30 \mathrm{ml}$ and last in $4 \mathrm{ml} 10 \%$ glycerol, containing $\mathrm{dH}_{2} \mathrm{O} .50 \mu \mathrm{l}$ aliquots were frozen in liquid nitrogen and stored at $-80^{\circ} \mathrm{C}$. During the whole procedure, solutions and cells were continuously kept on ice.

To transform cells, $0.5-1 \mu \mathrm{g}$ plasmid DNA and $50 \mu$ l of electrocompetent cells were transferred to a $1 \mathrm{~mm}$ electrode gap electroporation cuvette from Biozym Scientific $\mathrm{GmbH}$ (cat. no. 748010; Hessisch Oldendorf, Germany). An electric pulse was then delivered with the Electroporator 2510 (Eppendorf) at 1800 Volts. Subsequently, cells were collected in $1 \mathrm{ml}$ LB medium of which $100 \mu$ l was distributed on a $10 \mathrm{~cm}$ LB-Agar dish for overnight incubation. The following day single colonies were transferred to $5 \mathrm{ml}$ LB liquid cultures for 6 - 8 hours of incubation, after which the total volume was increased to $300 \mathrm{ml}$ for overnight incubation and until the $\mathrm{OD}_{600}$ ranged between 3 - 4. Cells were pelleted by centrifugation at $3320 \mathrm{rcf}$ and at $4{ }^{\circ} \mathrm{C}$ for $40 \mathrm{~min}$ and plasmids extracted with the NucleoBond Xtra Maxi kit (MN), according to the user manual instructions (MN March 2014/Rev. 12). In brief the extraction is based on $\mathrm{NaOH} / \mathrm{SDS}$ lysis of the cells (Birnboim and Doly, 1979), removal of cell debris, denatured proteins and chromosomal DNA by filtration and binding of plasmid DNA to, under acidic conditions, positively charged methyl-amino-ethanol residues on silica beads in the provided columns, while other contaminants are washed through. Plasmid DNA is eluted by neutralization of the positive charges in the columns under slightly alkaline conditions, precipitated with 2-propanol, washed with ethanol and resuspended in $\mathrm{dH}_{2} \mathrm{O}$ upon centrifugation. DNA yield and purity was determined with a Nanophotometer (Implen GmbH, Munich, Germany; www.implen.de).

\section{Vector quality control \& molecular cloning}

Received vectors were commonly tested by restriction digests for their expected restriction digest patterns and also sent for sequencing to verify the correct insert. For the production of AAVs the integrity of the insert-flanking ITRs is essential and was therefore assessed by performing Smal digests; Smal has two target sequences in each ITR. When vectors failed the initial verification process, new sources for the vectors were found and contact with collaborators providing flawed vectors was discontinued.

Standard mix for a restriction digest

Plasmid DNA-solution

$10 x$ restriction enzyme specific buffer

restriction enzyme $(10 \mathrm{U} / \mu \mathrm{l})$

$\mathrm{dH}_{2} \mathrm{O}$
$2-5 \mu g$

$2 \mu l$

$1 \mu \mathrm{l} / \mathrm{enzyme}$

up to a total of $20 \mu \mathrm{l}$

The reaction was then incubated at the enzyme's specific temperature for $2-3$ hours. 
50x Tris-Acetate-EDTA (TAE) buffer

$\begin{array}{lcl}\text { Tris }\left(\mathrm{C}_{4} \mathrm{H}_{11} \mathrm{NO}_{3}\right) & 242 \mathrm{~g} & \text { (Roth; cat. no. A411.1, M 121.14 g/mol) } \\ \text { Acetic acid }\left(\mathrm{C}_{6} \mathrm{H}_{4} \mathrm{O}_{2}\right) & 57.1 \mathrm{ml} & \text { (Roth; cat. no. 6755.2, M 60.05 g/mol) } \\ 0.5 \mathrm{M} \mathrm{EDTA}\left(\mathrm{pH} \mathrm{8.0,} \mathrm{C}_{10} \mathrm{H}_{16} \mathrm{~N}_{2} \mathrm{O}_{8}\right) & 100 \mathrm{ml} & \text { (Roth; cat. no. CN06.4, M } 292.25 \mathrm{~g} / \mathrm{mol} \text { ) } \\ \mathrm{dH}_{2} \mathrm{O} & \text { up to } 1 \mathrm{l} & \end{array}$

TAE buffer was prepared as a $50 x$ stock solution and diluted 50:1 with $\mathrm{dH}_{2} \mathrm{O}$ to final $40 \mathrm{mM}$ Tris, $20 \mathrm{mM}$ acetic acid and $1 \mathrm{mM}$ EDTA.

Agarose gel electrophoresis

Agarose gels consisted of $1.5 \%$ agarose (Invitrogen; cat. no. 16500-500) in 1x TAE buffer. $5 \mu$ l RotiGelStain (Roth; cat. no. 3865.2) was added for $100 \mathrm{ml}$ agarose gel to stain and detect DNA under UVlight. Samples were directly loaded with $6 \mu$ l 6x Loading Dye (Thermo Scientific; cat. no. R0611), containing bromphenol blue and xylene cyanol FF as visual references. 100 and 1,000 bp GeneRuler (ThermoScientific; cat. no. SM0243 \& SM1334) were loaded as aids to determine fragment sizes later. Electrophoresis was performed at 6 Volts/cm distance between the electrodes.

Sanger sequencing

To validate vector and insert sequences, Sanger sequencing was performed through GATC Biotech. Primers for the specific target sequences were designed with the help of the PrimerQuest Tool from Integrated DNA Technologies, Inc. (https://eu.idtdna.com/Primerquest/Home/Index) and produced by Sigma.

Sequences for primers binding in the promotor region

$\begin{array}{lll}\text { CMV } & \text { CGCAAATGGGCGGTAGGCGTG } & \text { (forward) } \\ \text { CaMKIl } \alpha & \text { GTTCTCCGTTTGCACTCAGG } & \text { (forward) } \\ \text { GFAP } & \text { CAGGTTGGAGAGGAGAC } & \text { (forward) } \\ \text { U6 } & \text { ACGATACAAGGCTGTTAG } & \text { (forward) }\end{array}$

Sequences for primers binding in the coding DNA sequence (CDS)

BDNF GCTGGCGATTCATAAGGATAGA (forward)

BDNF ATTCACGCTCTCCAGAGTCC (reverse)

hChR2 AAACTGAACATCGGCGGAAC (forward)

hChR2 CGTTCACCACCACAGGATTA (reverse)

EYFP/EGFP CTGAACTTGTGGCCGTTTAC (reverse)

Molecular cloning

Gel extraction

DNA fragments produced through restriction digest, were separated from the agarose gel using a scalpel and DNA was extracted using a QIAquick Gel Extraction Kit (QIAGEN; cat. no. 28704) according to the provided protocol. In brief, the DNA fragment containing gel is dissolved by application of a chaotropic salt-solution and heating to $50^{\circ} \mathrm{C}$, DNA is precipitated with 2-propanol 
and bound by silica membranes at $\mathrm{pH} \leq 7.5$, washed and subsequently eluted with an elution buffer at pH 8.5 (Borodina et al., 2003).

\section{DNA Ligation}

Ligations were performed using a T4 DNA Ligase (Thermo Scientific; cat. no. EL0011), following the recommendations of the User Guide provided by the Ligase manufacturer.

Ligation reaction:

$\begin{array}{ll}\text { Linear vector } & 100 \mathrm{ng} \\ \text { Insert DNA } & 300-500 \mathrm{ng} \\ \text { 10x T4 DNA Ligase Buffer } & 2 \mu \mathrm{l} \\ \text { T4 DNA Ligase } & 1 \mu \mathrm{l} \\ \mathrm{dH}_{2} \mathrm{O} & \text { to a total of } 20 \mu \mathrm{l}\end{array}$

To be able to express LSSmOrange under a cell-type specific promotor and prepare it for AAVmediated transduction, it was subcloned into pAAV-6P-noTB-SEWB and pAAV-GFAP2.2-EGFP-WPRE. EGFP was removed from those vectors and LSSmOrange was cut out from its original vector by digesting all three plasmids with BsHTI (Agel) and Notl (Thermo Scientific; cat. no. ER1462 \& ER0591). Fragments were separated through agarose gel electrophoresis and respective bands extracted, combined and ligated to yield pAAV-hsyn-LSSmOrange-WPRE and PAAV-GFAP2.2-LSSmOrangeWPRE.

To overexpress TrkB under synapsin promotor-control and get a LSSmOrange-tagged variant, Trkb-EGFP was subcloned into pAAV-hsyn-LSSmOrange-WPRE, by digesting with EcoRI and Notl (Thermo Scientific; cat. no. ER0271 \& ER0591) to get the EGFP variant, and EcoRI and BamHI (Thermo Scientific; cat. no. ER0271 \& ER0051) to cut away the EGFP and produce the fragments for the LSSmOrange variant. Fragments were separated through agarose gel electrophoresis and respective bands extracted, combined and ligated to produce the pAAV-hsyn-TrkB-EGFP/LSSmOrange vectors.

As indicated earlier, vectors and successful cloning was verified by restriction digests and Sanger sequencing. Sequences and maps were managed with ApE ('A plasmid Editor'; Wayne Davis, University of Utah, U.S.A.; version 2.0.47)

\subsection{Cell cultures}

For this dissertation the most frequently used model system to investigate optogenetic photocurrents and BDNF transfer was dissociated primary hippocampal cultures from E19 rats; these cultures consist mainly of excitatory pyramidal cells, are well characterized and provide a simple approach to study basic physiological functions (Banker and Cowan, 1977; Benson et al., 1994). However, organotypic hippocampal slice cultures from P4 rats were also prepared to study the transfer of fluorescently labeled BDNF in an intact circuit, where connectivity is well known (Schaffer, 1892). Both cultures were prepared from Wistar rats. Animals were bought from Charles River International Laboratories, Inc. by the animal core facility at the University Medical Center Göttingen, 
where they were housed and paired for breeding. All animal handling and experiments were conducted in accordance to relevant institutional guidelines and regulations of the German animal protection act.

Human embryonic kidney cells (HEK 293) bought from Stratagene (cat. no. 240073) and originally derived from embryonic adrenal precursor cells transformed with sheared adenovirus type 5 DNA by Alex van der Eb and Frank Graham in 1973 (Graham et al., 1977; Shaw et al., 2002), were used to produce AAV.

\subsubsection{Dissociated primary hippocampal cultures}

\section{Media \& Solutions}

Dissection medium

HBSS (Hank's Balanced Salt Solution)

(Gibco; cat. no. 14170-088)

Hepes (1 M)

$10 \mathrm{mM}$

(Gibco; cat. no. 15630-056)

Plating medium

DMEM (Dulbecco's modified Eagle media)

(Gibco; cat. no. 41966-029)

Fetal Bovine Serum (FBS)

$10 \%$

(Gibco; cat. no. 10500-064)

GlutaMax (200 mM L-alanyl-L-glutamine)

$2 \mathrm{mM}$

(Gibco; cat. no. 35050-038)

Penicillin 10,000 units/ml

100 units $/ \mathrm{ml}$

(Gibco; cat. no. 15140-122)

Streptomycin $10,000 \mu \mathrm{g} / \mathrm{ml}$

$100 \mu \mathrm{g} / \mathrm{ml}$

Culture medium

Neurobasal medium

(Gibco; cat. no. 21103-049)

B-27 Supplements (Brewer et al., 1993)

$1 \mathrm{ml} / 50 \mathrm{ml}$

(Gibco; cat. no. 17504-001)

GlutaMax (200 mM L-alanyl-L-glutamine)

$2 \mathrm{mM}$

(Gibco; cat. no. 35050-038)

Penicillin 10,000 units/ml

100 units/ml

(Gibco; cat. no. 15140-122)

Streptomycin $10,000 \mu \mathrm{g} / \mathrm{ml}$

$100 \mu \mathrm{g} / \mathrm{ml}$

\section{PEI coating}

Dissociated hippocampal cultures were cultured on $\varnothing-25 \mathrm{~mm}$ borosilicate cover glasses with a thickness of 1.5H (0.17 mm) Paul Marienfeld GmbH (cat. no. 0117650; Lauda-Königshofen, Germany; www.marienfeld-superior.com). Cover glasses were stored in $70 \%$ Ethanol and prior to plating, coated with $0.04 \%$ polyethylenimine (PEI) (Sigma; cat. no. P3143), which is an inexpensive substitute for polylysine and polyornithine, but works equally well for rat neuronal cultures (Ruegg and Hefti, 1984). Cover glasses were transferred to 6-well plates (CytoOne; cat. no. CC7672-7506). $1 \mathrm{ml} 1$ \% PEI stocks were made with $\mathrm{dH}_{2} \mathrm{O}$, stored at $-20{ }^{\circ} \mathrm{C}$, diluted further to $0.04 \%$ and for coating, $2 \mathrm{ml} \mathrm{PEI-}$ solution/cover glass was applied at room temperature for approximately 14 hours. Subsequently, 
coverslips were washed 3 times with $\mathrm{dH}_{2} \mathrm{O}$ and either directly used for culturing or kept in $\mathrm{dH}_{2} \mathrm{O}$ at $4{ }^{\circ} \mathrm{C}$ until needed.

\section{Preparation, plating and culturing}

The hippocampal culture protocol is an adaptation of the work of Gary A. Banker (Goslin and Banker, 1991; Kaech and Banker, 2006). Pregnant Wistar rats were sacrificed between E18 and E19 using combined $\mathrm{CO}_{2}$ and cervical dislocation. Embryos were extracted and brains transferred to $10 \mathrm{~cm}$ dishes containing dissection medium. Using forceps, hemispheres were separated, meninges removed, hippocampi extracted and collected in a separate $15 \mathrm{ml}$ falcon-tube on ice. Tissue was digested in $2 \mathrm{ml} 0.25 \%$ Trypsin-EDTA (Gibco; cat. no. 25200-056) for $20 \mathrm{~min}$ at $37^{\circ} \mathrm{C}$, subsequently washed 3 times with $5 \mathrm{ml}$ dissection medium and triturated in $1 \mathrm{ml}$ plating medium. Still existent tissue lumps were removed by filtration through a $100 \mu \mathrm{m}$ cell strainer (Corning, Inc.; cat. no. 352360) and yield was determined using a hemocytometer, excluding dead cells by trypan blue staining (Sigma; cat. no. T8154). Final concentration was adjusted and cells were plated at densities of 30,500 to 37,000 cells $/ \mathrm{cm}^{2}$ in $600 \mu$ l plating medium per cover glass and cultured at $37^{\circ} \mathrm{C}$ and $5 \% \mathrm{CO}_{2}$. The following day plating media was exchanged for $2 \mathrm{ml}$ culture medium. Cells were transfected with the appropriate expression construct using standard calcium phosphate transfection and/or transduced with AAV on DIV3. Electrophysiological recordings were performed from DIV8 onwards, imaging and immunocytochemical experiments took place around DIV14 - 16.

\section{Treatments}

$5 \mu \mathrm{M}$ AraC was added once on DIV7. Recombinant BDNF produced in bacteria or eukaryotic cells was applied at $100 \mathrm{nM} / \mathrm{ml}$ (Alamone Labs cat. no. B-250; R\&D Systems; cat. no. P23560) and BDNF scavenging TrkB immunoglobulin G (TrkB Ig-G) was applied at $0.4 \mu \mathrm{g} / \mathrm{ml}$ (Figurov et al., 1996; Shelton et al., 1995). Treatments were started on DIV3 and doses were repeated every 3 days. To increase neural network activity, extracellular $\mathrm{KCl}$ was increased by $5 \mathrm{mM}$ to a total of $10 \mathrm{mM}$, starting at DIV3 and for up to 9 days. Elevated $\mathrm{KCl}$ concentrations are commonly used for the stimulation of neuronal cultures, where increases in BDNF mRNA levels were reported; even though hippocampal cultures are sometimes kept in concentrations of up to $50 \mathrm{mM} \mathrm{KCl}$ for $6-48$ hours, no effects on the health of the cell culture were reported (Elliott et al., 1994; Zafra et al., 1990). For the chemical LTP protocol $10 \mu \mathrm{M}$ bicuculine, $200 \mu \mathrm{M}$ glycine, $100 \mathrm{~nm}$ rolipram and $50 \mu \mathrm{M}$ forskolin were applied once at DIV 3 6 and cells incubated for 9 - 12 days.

\section{Methods for expression and vector delivery}

Calcium phosphate transfection

Since first described in 1988 (Chen and Okayama, 1988), transfection with a DNA-calcium phosphate precipitate became a standard, highly-efficient technique to deliver expression vectors to cells in dissociated cell cultures. Our protocol is an adaptation for dissociated hippocampal cultures on 
$\emptyset$-25 mm cover glasses in 6-well plates of diverse protocols and optimizations that followed (Goetze et al., 2004; Jiang and Chen, 2006; Kohrmann et al., 1999; Xia et al., 1996).

Transfection buffer

$\mathrm{NaCl}$ (sodium chloride)

$274 \mathrm{mM} \quad$ (Roth; cat. no. P029.4, M $58.44 \mathrm{~g} / \mathrm{mol}$ )

$\mathrm{KCl}$ (potassium chloride)

$10 \mathrm{mM} \quad$ (Roth; cat. no. 6781.1, M $74.56 \mathrm{~g} / \mathrm{mol}$ )

$\mathrm{Na}_{2} \mathrm{HPO}_{4}$ (di-sodium hydrogenphosphate)

$1.4 \mathrm{mM} \quad$ (Roth; cat. no. T876.2, M $141.96 \mathrm{~g} / \mathrm{mol}$ )

$\mathrm{D}(+)$-Glucose $\left(\mathrm{C}_{6} \mathrm{H}_{12} \mathrm{O}_{6}\right)$

$15 \mathrm{mM} \quad$ (Roth; cat. no. HN06.3, M $180.16 \mathrm{~g} / \mathrm{mol}$ )

Hepes $\left(\mathrm{C}_{8} \mathrm{H}_{18} \mathrm{~N}_{2} \mathrm{O}_{4} \mathrm{~S}\right)$

$42 \mathrm{mM} \quad$ (Roth; cat. no. HN77.4, M $283.31 \mathrm{~g} / \mathrm{mol}$ )

in $\mathrm{dH}_{2} \mathrm{O}$

Titrated with $1 \mathrm{M} \mathrm{NaOH}$ to $\mathrm{pH}$ of $7.05-7.12$ and sterile filtered with $0.22 \mu \mathrm{m}$ vacuum-driven filters.

$2 \mathrm{M} \mathrm{CaCl}_{2}$ solution

$\mathrm{CaCl}_{2}$ (calcium chloride)

$2 \mathrm{M}$

(Roth; cat. no. HN04.3, M $147.02 \mathrm{~g} / \mathrm{mol}$ )

in $\mathrm{dH}_{2} \mathrm{O}$

Transfection mix for single $\varnothing$-25 mm hippocampal culture

$2 \mathrm{M} \mathrm{CaCl}_{2}$ solution

$7.5 \mu \mathrm{l}$

Plasmid DNA

$3.5-4 \mu \mathrm{g}$

$\mathrm{dH}_{2} \mathrm{O}$

added up to a total of $60 \mu \mathrm{l}$

Transfection buffer

$60 \mu \mathrm{l}$

To make the transfection mix, $\mathrm{CaCl}_{2}$ solution, $\mathrm{dH}_{2} \mathrm{O}$ and plasmid DNA are thoroughly mixed and transfection buffer is subsequently added drop-wise, while the tube is continuously gently shaken. Before application the finished transfection mix is incubated for $20 \mathrm{~min}$ at room temperature, to allow the calcium phosphate precipitate formation to take place.

Conditioned culture medium was collected and exchanged for pre-equilibrated Opti-MEM (Gibco; cat. no. 11058-021), 1/3 ${ }^{\text {rd }}$ fresh culture medium was added, the mix sterile filtered using $0.22 \mu \mathrm{m}$ syringe-driven filters (Millipore; cat. no. SLGV033RS) and stored under cell culture conditions until needed. Per well, $120 \mu$ transfection mix was gently distributed over the culture and the culture was put back into the incubator for $90 \mathrm{~min}$. Afterwards the transfection mix containing Opti-MEM was exchanged for $37^{\circ} \mathrm{C}$ and $10 \% \mathrm{CO}_{2}$ pre-equilibrated Neurobasal medium, followed by another $10 \mathrm{~min}$ of incubation under culturing conditions and subsequent exchange for the conditioned medium to remove the precipitate.

If cell health is affected or less efficient transfection is wanted, incubation times or transfection buffer concentration in the transfection mix can be decreased to $40-60 \mathrm{~min}$ or $75-80 \%$ respectively.

AAV-mediated transduction

In order to express certain constructs in large quantities of a specific cell type, we made use of AAV serotype 1 \& 2 chimeric virions (Hauck et al., 2003) (see HEK 293 cell section for further detail). Of 
the harvested supernatant $1-2 \mu \mathrm{l}$ were applied per well and directly to the culture media of dissociated hippocampal cultures at DIV3. These chimeric virions successfully transduce both neurons and astrocytes and the observed transduction efficiency of $>80 \%$, detected by the neuronal (synapsin) and astrocyte (GFAP2.2) promoter-specific expression of fluorophores was consistent with that reported in the literature (Royo et al., 2008). Purified AAV serotype 6 was applied at $10^{6}$ virions per $\varnothing-25 \mathrm{~mm}$ culture. This was sufficient to drive strong and ICC detectable expression and was low enough to avoid toxic effects, that are reported to occur at much higher doses (Royo et al., 2008).

\section{Electroporation}

For vectors where expression using AAV was too low, a more widespread expression throughout the culture was wanted or for which constructs were too large to be put into AAVs, electroporation can be used to deliver the target vector. This was done for TrkB-LSSmOrange, LSSmOrange control construct, and subcellular marker proteins (EEA1, Rab5a, Rab7a, Lamp1 and LC3). Electroporation was done using a Rat Neuron Nucleofector Kit (Lonza; cat. no. VPG-1003) and the corresponding Nucleofector 2b Device (Lonza; cat.no. AAB-1001), by following the recommendations of the protocol provided by the manufacturer. In short, dissociated hippocampal preparation was done as described earlier, but stopped before plating. $3-5 \times 10^{6}$ cells were pelleted at room temperature and $70 \mathrm{rcf}$ for $5 \mathrm{~min}$, followed by resuspension in $100 \mu \mathrm{l}$ of the provided Nucleofector solution, addition of $3 \mu \mathrm{g}$ plasmid DNA and transfer to the certified cuvettes, also provided with the kit. For transfection of both cell types, neurons and astrocytes, the Nucleofector program 0-003 (for primary rat hippocampal or cortical neurons and high cell viability) was used. Subsequently, the cells were collected in $500 \mu \mathrm{l}$ pre-equilibrated plating medium and plated as described in the section about culturing, but, because of the increased cell lethality of this procedure, approximately $\geq 10^{5} \mathrm{cells} / \mathrm{cm}^{2}$ were plated in $600 \mu \mathrm{l}$ plating medium. To remove Nucleofector solution remnants, plating medium was exchanged after 6 hours for $2 \mathrm{ml}$ plating medium and the following day for culture medium.

\subsubsection{Organotypic hippocampal brain slices (OHBS)}

To verify transfer of BDNF-mRFP1 under more physiological conditions and investigate directionality of BDNF transfer and release from pre- or postsynaptic sites, rat OHBS were used as model, where connectivity is well studied and preserved during preparation. Organotypic hippocampal slices are ideal for this approach, because from the CA3 area the Schaffer collaterals, CA3 pyramidal cell axons, traverse to and innervate the pyramidal cells in the CA1 area (Schaffer, 1892). 
OHBS culture medium

MEM (Minimal Essential Medium) $\quad 50 \%$

(Gibco; cat. no. 11090-081)

HBSS (Hank's Balanced Salt Solution) $\quad 25 \%$

(Gibco; cat. no. 14170-088)

Horse serum

$25 \%$

(Sigma; cat. no. H1138)

GlutaMax (200 mM L-alanyl-L-glutamine)

$2 \mathrm{mM}$

(Gibco; cat. no. 35050-038)

$\mathrm{D}(+)$-Glucose $\left(\mathrm{C}_{6} \mathrm{H}_{12} \mathrm{O}_{6}\right)$

$6.5 \mathrm{mg} / \mathrm{ml}$ (Roth; cat. no. HN06.3, M $180.16 \mathrm{~g} / \mathrm{mol}$ )

Pencillin 10,000 units/ml

100 units/ml

(Gibco; cat. no. 15140-122)

Streptomycin $10,000 \mu \mathrm{g} / \mathrm{ml}$

$100 \mu \mathrm{g} / \mathrm{ml}$

Based on the original recipe for Filter Culture Medium published at Cold Spring Harbor Protocols:

(http://cshprotocols.cshlp.org/content/2007/10/pdb.rec11167)

Preparation

P4-Wistar rats were decapitated and brains transferred to $10 \mathrm{~cm}$ dishes containing dissection medium enriched with $6.5 \mathrm{mg} / \mathrm{ml} \mathrm{D(+)-Glucose.} \mathrm{Using} \mathrm{forceps} \mathrm{and} \mathrm{periodontal} \mathrm{probes,} \mathrm{hemispheres}$ were separated, meninges removed, hippocampi extracted and collected in a separate $10 \mathrm{~cm}$ dish containing glucose enriched dissection medium. $400 \mu \mathrm{m}$ thick coronal slices were prepared using a manual tissue slicer (Stoelting Co. Europe, Dublin, Ireland; cat. no. 51425 equipped with Astra Superior Platinum razor blades). Slices were selected based on their appearance under the stereotactic microscope and $2-3$ slices were transferred on $\varnothing-30 \mathrm{~mm}$ hydrophilic polyterafluorethylene membranes with $0.4 \mu \mathrm{m}$ pores (Millipore; cat.no. PICMORG50) in 6-well plates, filled with $1 \mathrm{ml}$ OHBS medium. Membranes are floating on top of the medium so that membrane and slices are provided with nutrients through the medium by diffusion and oxygen by direct gas exchange with the air (Stoppini et al., 1991); slices should not be submerged in medium. Medium was changed once on the day following the dissection and then every 2 - 3 days.

AAV injection was done at DIV1 after medium change. Injection pipettes were made from borosilicate capillaries (Drummond Scientific Company, Broomall, U.S.A.; cat. no. 3-000-203-G/X, OD $1.14 \mathrm{~mm} \times$ ID $0.53 \mathrm{~mm}$ ) by pulling them with a P-97 Micropipette Puller, equipped with a $3 \times 3 \mathrm{~mm}$ box filament (Sutter Instruments Co.; cat. no. P-97 \& FB330B). This procedure is similar to that for microinjection pipettes provided in the user manuals. Pipettes were broken at their tip with forceps to produce injection pipettes with diameters well below $1 \mathrm{~mm}$. Pipettes were backfilled with crude AAV1/2 extract or purified AAV6, using $20 \mu \mathrm{l}$ Microloader pipette tips (Eppendorf; cat. no. 5242 956.003) and slices were injected in CA3 or CA1 areas, identified by morphology, using a FemtoJet express 5248 (Eppendorf; cat. no. 92001 0521) at $550 \mathrm{p}_{\mathrm{i}}$ (injection pressure) for 3 - $7 \mathrm{~s}$ to deliver volumina in the range of estimated 50-100 nl. Successful expression was checked the following days with a stereotactic microscope equipped with a HXP $120 \mathrm{C}$ Illuminator (Zeiss) and appropriate filters. 


\subsubsection{HEK 293 cultures}

HEK 293 cell medium

DMEM (Dulbecco's modified Eagle media)

(Gibco; cat. no. 41966-029)

Fetal Bovine Serum (FBS)

$10 \%$

(Gibco; cat. no. 10500-064)

Pencillin 10,000 units/ml

100 units/ml

(Gibco; cat. no. 15140-122)

Streptomycin $10,000 \mu \mathrm{g} / \mathrm{ml}$

$100 \mu \mathrm{g} / \mathrm{ml}$

HEK 293 cell culturing

HEK 293 cells were cultured in $10 \mathrm{~cm}$ cell culture dishes (Greiner Bio-One; cat. no. 664160) with $10 \mathrm{ml}$ HEK 293 cell medium at $37{ }^{\circ} \mathrm{C}$ and $5 \% \mathrm{CO}_{2}$. Upon reaching a $70-80 \%$ confluence, culture was passaged and split at 1:5/10 for transfection on the following day or 1:25 for maintaining the culture. For splitting, HEK 293 cells were washed once with $37^{\circ} \mathrm{C}$ warm DPBS (Gibco; cat. no. 14190-094) and dissociated from the culture dish by treatment with $1 \mathrm{ml} 0.25 \%$ Trypsin-EDTA (Gibco; cat. no. 25200056) at $37^{\circ} \mathrm{C}$. The cell suspension was collected in HEK 293 cell medium and pelleted at $700 \mathrm{rcf}$ for $3 \mathrm{~min}$. Pelleted cells were resuspended in fresh HEK 293 cell medium and appropriate dilutions were brought onto new culture dishes. For long-term storage, dishes at $80 \%$ confluence were pelleted and per dish, cells were resuspended in $1 \mathrm{ml} \mathrm{FBS}$ containing $10 \%$ DMSO (Sigma; cat. no. 276855) and glycerol (Roth; ca. no. 3783.1). $1 \mathrm{ml}$ aliquots in cryotubes were then transferred to a 2-Propanol freezing container for slow freezing (Thermo Scientific/Nalgene; cat. no. 5100-0001), frozen and subsequently stored at $-80^{\circ} \mathrm{C}$. For starting a culture, one of these cryostocks was thawed and diluted with $10 \mathrm{ml}$ HEK 293 cell medium, pelleted and again resuspended in HEK 293 cell medium, before plating, to remove DMSO.

\section{Generation of recombinant adeno-associated virus (AAV) in HEK 293 cells}

Recombinant chimeric AAV serotype 1 \& 2 particles were generated with the use of HEK 293 cells, by transfecting HEK 293 cells with vectors encoding AAV sequences for the generation of the viral particles and the vector containing the sequence that the AAVs are supposed to deliver, flanked by two internal tandem repeats (ITR), that are important for the packaging process (McClure et al., 2011). Different serotypes have different target tissue or can handle larger genes for packaging better than others. These can be mixed by delivering vectors with the different specific replication and capsid sequences, the chimeric variants hold mixed characteristics from the contributing serotypes; especially the serotype $1 \& 2$ hybrids were shown to be efficient in transducing neurons and astrocytes (Allocca et al., 2008; Hauck et al., 2003; Royo et al., 2008).

HEK 293 cells were grown until a density of $60 \%$ was reached and transfected with the vector plasmid, a 50:50 ratio of plasmids for AAV1 (pH21) and AAV2 (pRV1) replication and capsid sequences and the adenovirus helper-plasmid ( $\mathrm{FF} \Delta 6$ ), using an adapted calcium phosphate transfection protocol for $10 \mathrm{~cm}$ dishes $(80 \mu \mathrm{g}$ total plasmid DNA, $50 \%$ pF $\Delta 6,25 \%$ expressionvector, $12.5 \% \mathrm{pH} 21$ and pRV1). Important differences are, that prior to transfection HEK 293 cells were gently washed once with $37^{\circ} \mathrm{C}$ warm DPBS (Gibco; cat. no. 14190-094), which was exchanged for 
fresh HEK 293 cell medium and that calcium phosphate precipitate was not removed, but left in the culture for at least 48 - 72 hours. Successful transfection could often be observed by fluorescence; most constructs, even though equipped with a CaMKIl $\alpha$, synapsin or GFAP promotor, were weakly expressed in HEK 293 cells. Cell culture health was continuously checked during this time and if medium $\mathrm{pH}$ dropped, indicated by the phenol red $\mathrm{pH}$ indicator contained in the medium, medium was gently exchanged. Cells were kept as long as possible in culture to maximize the time cells produce viral particles and therefore increase yield. In case cells grew too dense and started detaching, or after $48-72$ hours post transfection, cells were harvested in $500 \mu$ IPBS and lysed by 4 repeated freezing $\left(-80^{\circ} \mathrm{C}\right)$ and thawing $\left(37^{\circ} \mathrm{C}\right)$ cycles. Cell debris was removed by centrifugation at $10,000 \mathrm{rcf}$ for $5 \mathrm{~min}$. Supernatant containing a crude AAV extract was transferred to $1.5 \mathrm{ml}$ tubes, could be stored at $4{ }^{\circ} \mathrm{C}$ for months and can be directly used for application to dissociated hippocampal cultures or injection into OHBS.

\subsection{Experiments \& data acquisition}

\subsubsection{Immunocytochemistry (ICC)}

Immunocytochemistry generally describes the detection of specific antigens via binding of an antigen-specific antibody, which either directly or indirectly can be detected with dyes or enzymes connected to the antibodies. Since the first use of dye-coupled antibodies for the detection of a specific antigen (Coons et al., 1941), this technique became a common laboratory method for the detection of subcellular or cell-type specific antigens. Most commonly the indirect detection, detecting a primary antibody bound to the specific target antigen with a second fluorescent probe coupled antibody is used.

10x Phosphate buffered saline (PBS)

$\mathrm{NaCl}$ (sodium chloride)

$80 \mathrm{~g} \quad$ (Roth; cat. no. P029.4, M $58.44 \mathrm{~g} / \mathrm{mol}$ )

$\mathrm{KCl}$ (potassium chloride)

$2 \mathrm{~g} \quad$ (Roth; cat. no. 6781.1, M $74.56 \mathrm{~g} / \mathrm{mol}$ )

$\mathrm{Na}_{2} \mathrm{HPO}_{4}$ (di-sodium hydrogen phosphate) $\quad 14.4 \mathrm{~g}$

(Roth; cat. no. T876.2, M $141.96 \mathrm{~g} / \mathrm{mol}$ )

$\mathrm{KH}_{2} \mathrm{PO}_{4}$ (potassium dihydrogen phosphate)

$2.4 \mathrm{~g}$

(Merck; cat. no. 104873, M $136.08 \mathrm{~g} / \mathrm{mol}$ )

in $\mathrm{dH}_{2} \mathrm{O}$

Stock solution was adjusted to a $\mathrm{pH}$ of 7.4 with $1 \mathrm{M} \mathrm{NaOH}$ and subsequently sterile-filtered using a vacuum-driven filter. Further $2 x$ and $1 x$ dilutions were made using $\mathrm{dH}_{2} \mathrm{O}$.

0.2 M Phosphate buffer

$\mathrm{NaH}_{2} \mathrm{PO}_{4} \cdot 2 \mathrm{H}_{2} \mathrm{O}$ (sodium dihydrogen phosphate) $38 \mathrm{mM} \quad$ (Roth; cat. no. 2370.3, M $156.01 \mathrm{~g} / \mathrm{mol}$ )

$\mathrm{Na}_{2} \mathrm{HPO}_{4}$ (di-sodium hydrogen phosphate) $\quad 162 \mathrm{mM} \quad$ (Roth; cat. no. T876.2, M $141.96 \mathrm{~g} / \mathrm{mol}$ )

$8 \%$ Paraformaldehyde solution

$\begin{array}{lll}\left.\text { Paraformaldehyde }\left(\mathrm{PFA}, \mathrm{CH}_{2} \mathrm{O}\right)_{\mathrm{n}}\right) & 8 \%(40 \mathrm{~g} / 500 \mathrm{ml}) \quad \text { (Roth; cat. no. 0335.3) }\end{array}$

in $\mathrm{dH}_{2} \mathrm{O}$ 
To dissolve PFA, dH $\mathrm{d}_{2} \mathrm{O}$ was kept at $65{ }^{\circ} \mathrm{C}$ and $15-20$ drops $5 \mathrm{M} \mathrm{NaOH}$ were added. $4 \%$ PFA solution was produced by 50:50 dilution with $0.2 \mathrm{M}$ phosphate buffer.

Blocking buffer

Donkey serum

$2 \%$

(Sigma; D9663)

Triton-X

$0.1 \%$

(Roth; cat. no. 3051.2)

$\mathrm{NaN}_{3}$ (sodium azide)

$0.05 \%$

(Roth; cat. no. K305.1)

in $2 x$ PBS

Primary antibodies

antigen | host spec. | dilution | provider (cat. no.)

ALDH1L1 mouse 1:500 NeuroMab (75-164)

BDNF mouse

1:100 DSHB \#9

$\begin{array}{llll}\text { EEA1 rabbit } & 1: 300 & \text { R. Jahn, Dr. (MPI:bpc) }\end{array}$

GFAP guinea pig 1:750 Synaptic Sys. $(173004)$

GFP mouse $1: 1000$

GFP rabbit $1: 1000$

Iba1 rabbit 1:100

Lamp1 mouse 1:100

Map2 mouse 1:2000 Millipore (MAB3418)

NG2 rat $1: 100$

Rab5 mouse $\quad 1: 100 \quad$ R. Jahn, Dr. (MPI:bpc)

Rab7 rabbit $1: 100 \quad$ R. Jahn, Dr. (MPI:bpc)

RFP rat 1:1000 ChromoTek (5F8)

Syt-7 rabbit 1:500 Abcam (ab76274)
Secondary antibodies

Fluorph. | antigen | host | provider (cat. no.)

AF488 mouse donkey A-21202

AF488 rabbit goat A-11008

AF488 rat donkey A-21208

AF546 rat donkey A-11081

AF647 mouse donkey A-31571

AF647 mouse goat A-21235

AF647 rabbit goat A-21244

I-VAM-EN001-D)

AF647 guinea pig goat A-21450

AF647 rat goat A-21247

Procedures

Cells were fixed using $2 \mathrm{ml} 4$ \% PFA for $30 \mathrm{~min}$, washed 3 times with DPBS (Gibco) to remove PFA and samples were blocked and permeabilized by incubation in $1 \mathrm{ml}$ blocking buffer for $30 \mathrm{~min}$. Primary antibody was applied in $1 \mathrm{ml}$ blocking buffer in the above stated dilutions and kept overnight at $4{ }^{\circ} \mathrm{C}$. Subsequently, cells were washed gently 3 times with DPBS (Gibco), before fluorescently labeled secondary antibodies were applied in $1 \mathrm{ml}$ blocking buffer in the above stated dilutions for 2 hours at room temperature. Cells were washed 3 more times with DPBS (Gibco) and coverslips were mounted on imaging slides with Fluoromount (Sigma; cat. no. F4680). Slides were imaged on a Zeiss LSM710 confocal microscope using $20 x, 40 x$ or $63 x$ objectives (see microscopy section for details).

To investigate whether BDNF is being internalized, live labeling of BDNF using preconjugated primary and secondary antibodies was performed. Therefore, undiluted primary antibody for RFP or GFP detection, was mixed with the respective fluorescently labeled secondary antibody, incubated for $30 \mathrm{~min}$ at room temperature and was applied in fresh culture medium at standard working dilutions to the cells for another 30 mins, before they were imaged. 


\subsubsection{Microscopy}

$50 \mathrm{mM}$ Ammonium chloride solution:

$\mathrm{NaCl}$ (sodium chloride)

$90 \mathrm{mM} \quad$ (Roth; cat. no. P029.4, M $58.44 \mathrm{~g} / \mathrm{mol}$ )

$\mathrm{KCl}$ (potassium chloride)

$5 \mathrm{mM} \quad$ (Roth; cat. no. 6781.1, M $74.56 \mathrm{~g} / \mathrm{mol}$ )

$\mathrm{CaCl}_{2}$ (calcium chloride)

$2 \mathrm{mM}$

(Roth; cat. no. HN04.3, M $147.02 \mathrm{~g} / \mathrm{mol}$ )

$\mathrm{MgCl}_{2}$ (magnesium chloride)

$2 \mathrm{mM}$

$\mathrm{NH}_{4} \mathrm{Cl}$ (ammonium chloride)

$50 \mathrm{mM}$

Hepes $\left(\mathrm{C}_{8} \mathrm{H}_{18} \mathrm{~N}_{2} \mathrm{O}_{4} \mathrm{~S}\right)$

$20 \mathrm{mM}$

(Roth; cat. no. KK36.3, M $95.22 \mathrm{~g} / \mathrm{mol}$ )

$\mathrm{D}(+)$-Glucose $\left(\mathrm{C}_{6} \mathrm{H}_{12} \mathrm{O}_{6}\right)$

$5.5 \mathrm{mM}$

(Roth; cat. no. P726.1, M $53.49 \mathrm{~g} / \mathrm{mol}$ )

in $\mathrm{dH}_{2} \mathrm{O}$

Imaging buffers \& imaging

Most imaging was done with living samples. For this, $\varnothing-25 \mathrm{~mm}$ cover glasses were transferred into a low profile RC-41LP imaging chamber from Warner Instruments, LLC (Hamden, U.S.A.; cat. no. 640368) with a bottom part suitable to hold $\varnothing-25 \mathrm{~mm}$ cover glasses and chambers were filled with $1 \mathrm{ml}$ of an imaging buffer, which was principally the same as the extracellular solution for doing electrophysiology (refer to the electrophysiology section below for contents), but the $\mathrm{KCl}$ concentration was increased to $5 \mathrm{mM}$ and $\mathrm{NaCl}$ concentration was adjusted accordingly to 145.4 mM. For experiments testing acidic quenching of BDNF-EGFP a $50 \mathrm{mM}$ ammonium chloride solution was prepared and mixed with the imaging buffer to equal proportions to reach a final $\mathrm{NH}_{4} \mathrm{Cl}$ concentration of $25 \mathrm{mM}$.

Most live imaging experiments that are described in this thesis were conducted using a Zeiss LSM710 confocal microscope, with 405, 458, 488, 561 and $633 \mathrm{~nm}$ lasers, with a W Plan-Apochromat 20x water immersion objective (Zeiss; cat. no. 421452-9880); membranes with OHBS were cut from their holding structure, transferred to an imaging slide and kept in imaging buffer between the slide and a cover glass. OHBS were imaged using Plan-APOCHROMAT 10x or 20x objectives (Zeiss; cat. no. 420640-9900 \& 420650-9901). Image acquisition was controlled with the provided Zen Black software; software intrinsic algorithms determined optimal imaging parameters, laser line, filters, detector ranges, scanning speed and resolution, based on the used fluorophores and objectives. Laser intensity and gain was adjusted with the help of the range indicator. 8-bit images were considered sufficient for most images meant for quantitative analysis. Unless specifically indicated, images were acquired and saved as 8-bit images in the Zeiss microscope file format CZI, preserving the provided metainformation. Within the separate experiments imaging conditions were kept consistent.

Fixed and mounted samples were imaged using the same microscope, equipment and procedures. However, for some experiments $40 \mathrm{x}$ and $63 \mathrm{x}$ Plan-APOCHROMAT oil immersion objectives were used together with $518 \mathrm{f}$ immersion oil (Zeiss; cat. no. 420762-9800, 420782-9900 \& 444960). 
Image analysis and handling

Image files were handled and analyzed with the Open Source and public domain software ImageJ (Wayne Rasband, National Institutes of Health, U.S.A.; version 1.50e) and the derived imageprocessing package Fiji ('Fiji is just ImageJ', version 2.0.0-rc-43/1.50e). Colocalization was tested using the provided Coloc 2 plugin. For this, backprojected pinhole radii and PSF for 20x W Plan (1332.42 nm \& 2D $4.88 \mu \mathrm{m})$ and 40x (710.63 nm \& 2D $3.75 \mu \mathrm{m})$ Plan objectives were calculated using the PSF calculator (Scientific Volume Imaging B.V., Hilversum, Netherlands; https://svi.nl/forms/pinhole.php; https://svi.nl/NyquistCalculator). Data handling, calculations and graphs were done using Microsoft Excel for Mac 2011 (Microsoft; version 14.6.0) and statistical analysis was done and representative graphs were created using Prism (GraphPad Sofware, Inc., La Jolla, U.S.A.; version 6). For display images and graphs were combined in figures, and annotations were added using Adobe Illustrator CS5 (Adobe Systems, Inc., San José, U.S.A.; version 15.1.0).

\subsubsection{Optogenetics and electrophysiology}

Extracellular solution

$\mathrm{NaCl}$ (sodium chloride)

$\mathrm{KCl}$ (potassium chloride)

$\mathrm{CaCl}_{2}$ (calcium chloride)

$\mathrm{MgCl}_{2}$ (magnesium chloride)

Hepes $\left(\mathrm{C}_{8} \mathrm{H}_{18} \mathrm{~N}_{2} \mathrm{O}_{4} \mathrm{~S}\right)$

$\mathrm{D}(+)$-Glucose $\left(\mathrm{C}_{6} \mathrm{H}_{12} \mathrm{O}_{6}\right)$

in $\mathrm{dH}_{2} \mathrm{O}$

$\begin{aligned} 148 \mathrm{mM} & \text { (Roth; cat. no. P029.4, M } 58.44 \mathrm{~g} / \mathrm{mol}) \\ 2.4 \mathrm{mM} & \text { (Roth; cat. no. 6781.1, M } 74.56 \mathrm{~g} / \mathrm{mol}) \\ 2 \mathrm{mM} & \text { (Roth; cat. no. HN04.3, M } 147.02 \mathrm{~g} / \mathrm{mol}) \\ 1 \mathrm{mM} & \text { (Roth; cat. no. KK36.3, M } 95.22 \mathrm{~g} / \mathrm{mol}) \\ 10 \mathrm{mM} & \text { (Roth; cat. no. HN77.4, M } 238.31 \mathrm{~g} / \mathrm{mol}) \\ 7 \mathrm{mM} & \text { (Roth; cat. no. HN06.3, M } 180.16 \mathrm{~g} / \mathrm{mol})\end{aligned}$

$148 \mathrm{mM} \quad$ (Roth; cat. no. P029.4, M $58.44 \mathrm{~g} / \mathrm{mol}$ )

(Roth; cat. no. HN06.3, M $180.16 \mathrm{~g} / \mathrm{mol}$ )

The osmolality should be around $300 \mathrm{mOsm}$ and $\mathrm{pH}$ was adjusted to 7.4 with $10 \mathrm{M} \mathrm{NaOH}$. If required specific channel blockers were added.

Internal solution

potassium gluconate

$130 \mathrm{mM} \quad$ (Sigma; cat. no. P1847, M $234.25 \mathrm{~g} / \mathrm{mol}$ )

$\mathrm{KCl}$ (potassium chloride)

$8 \mathrm{mM} \quad$ (Roth; cat. no. 6781.1, M $74.56 \mathrm{~g} / \mathrm{mol}$ )

$\mathrm{CaCl}_{2}$ (calcium chloride)

$2 \mathrm{mM} \quad$ (Roth; cat. no. HN77.4, M $283.31 \mathrm{~g} / \mathrm{mol}$ )

$\mathrm{MgCl}_{2}$ (magnesium chloride)

$1 \mathrm{mM} \quad$ (Roth; cat. no. KK36.3, M $95.22 \mathrm{~g} / \mathrm{mol}$ )

EGTA

$10 \mathrm{~m} \quad$ (Sigma; cat. no. P1847, M $234.25 \mathrm{~g} / \mathrm{mol}$ )

Hepes $\left(\mathrm{C}_{8} \mathrm{H}_{18} \mathrm{~N}_{2} \mathrm{O}_{4} \mathrm{~S}\right)$

$10 \mathrm{mM}$ (Roth; cat. no. HN77.4, M $238.31 \mathrm{~g} / \mathrm{mol}$ )

Mg-ATP

$2 \mathrm{mM}$ (Sigma; cat. no. A9187, M 507.18 g/mol)

GTP-Na

$0.3 \mathrm{mM}$ (Sigma; cat. no. G8877, M $523.18 \mathrm{~g} / \mathrm{mol}$ )

Osmolality should be between $290-295 \mathrm{mOsm}$. pH was adjusted to 7.3 with $1 \mathrm{M} \mathrm{KOH}$.

Optogenetic \& electrophysiological experiments

All optogenetic experiments in this dissertation were conducted on an inverted Zeiss Axio Observer.Z1 equipped with a laser module providing a $405 \mathrm{~nm} 50 \mathrm{~mW}$ diode laser, $488 \mathrm{~nm} 100 \mathrm{~mW}$ 
OPSL, $561 \mathrm{~nm} 40 \mathrm{~mW}$ diode laser and $639 \mathrm{~nm} 30 \mathrm{~mW}$ diode laser; a 20x LD A-Plan (cat. no. 4412419910), 40x and 63x EC Plan-NEOFLUAR (cat. no. 420460-9900 \& 420480-9900) and 100x $\alpha$ PlanAPOCHROMAT (cat. no. 420792-9800); a TIRF slider module (cat. no. 423683-9021), a laser manipulation module ('Direct FRAP'; cat. no. 423635-9000) and an Evolve 512 EMCCD camera (Photometrics, Tucson, U.S.A.). The AxioObserver.Z1 was custom-modified with the help of Rapp OptoElectronic GmbH ('ROE'; Hamburg, Germany). An additional 594 nm laser (DL594 594 nm $100 \mathrm{~mW}$ (DPSS laser)) was added and brought into the epi-fluoresence light path with a $940 \mu \mathrm{m}$ light guide Figure $2 \mathrm{a}$, together with custom photomasks, complementing the already existing DirectFRAP photomasks by adding a $594 \mathrm{~nm}$ border ( $2 \mu \mathrm{m}$ for $100 \mathrm{x}$ objective) around the DirectFRAP photomasks (Figure $2 \mathrm{~b}, \mathrm{c}$ ). ROE photomask \#1, providing illumination of a $150 \mu \mathrm{m}$ wide area with the $100 x$ objective (Figure $2 \mathrm{c}$ ) or no photomask was used for initial wavelength response tests. More positions for DirectFRAP and ROE photomasks exist, but were not used in this dissertation. System lasers and DL594 were operated by TTL pulses delivered by the HEKA amplifier to the generic digital in port at the SVB1 component or directly to the acousto-optic modulator of the DL594 (Figure 2d). The purpose of generic digital in ports was defined using the software controlling the microscope components to design custom imaging procedures, delivering light aligned to the recording procedure. The measured channel properties and observed effects of simultaneous illumination were well within reasonable limits and conserved throughout related channel variants. We therefore reason, that the results are still meaningful to draw conclusions about the individual channel properties. In addition, because we did not use a specialized setup for electrophysiological recordings and combined optical stimulation, where all the components would work perfectly syntonic and are adjusted to the experimental conditions, we were depending on a ready-made conventional TIRFlaser microscope hardware and software controlling the laser module with the 405, 488, 561 and $639 \mathrm{~nm}$ lasers. The amplifier was operated using a second computer running the software for the electrophysiological recordings, simultaneously orchestrating the laser stimulations by TTL trigger pulses delivered by the amplifier to control the DL594 auto occustic mirror and trigger the SVB1 microscope signal distribution box of the TIRF microscope. This complex stimulation and recording apparatus was the origin for inaccuracies and delays in the signaling and hence, control and timing of the laser pulses. Consequently, some recordings exhibit tiny, but nevertheless noticeable shifts in illumination onset, duration and stop of the single wavelength or simultaneous illumination. This is only a cosmetic problem and should have no influence on the significance of the here reported findings.

Laser powers were determined with a laser power meter (Fieldmate) and a silicon optical sensor (OP-2 VIS) from Coherent GmbH (cat.no. 1098297 \& 1098313; Dieburg, Germany; www.coherent.de) at the back aperture of the $40 x$ and $100 x$ objective, using two aluminum masks to represent the respective exit pupil diameter, 10.7 and $4.8 \mathrm{~mm}$. However, there was no difference in the measured power between those two masks. Laser intensities were measured after $4-6$ hours of use and with the TIRF/FRAP beamsplitter set at $50 \%$ TIRF/50 \% FRAP. The measured laser powers 
were subsequently corrected for the respective transmission of the objective ( $\sim 90 \%$ and $\sim 70 \%$ ). Laser output generally fluctuated $\pm 30 \mu \mathrm{W}$ and the measurement error was previously determined to be around $10 \%$. DL594 was measured using the $200 \mu \mathrm{m}$ light guide and respective powers for the $100 \mu \mathrm{m}$ light guide were extrapolated. Because of all these factors influencing the results, the reported power densities do not represent exact values, but estimates.

Channelrhodopsins were characterized by their responses to $500 \mathrm{~ms}$ light pulses of 405,488 , 561, 594 and $639 \mathrm{~nm}$, using the DL594 and no ROE photomask for the $594 \mathrm{~nm}$ and the TIRF laser for the other wavelengths. For the recording of photocurrents from channels with slow-photocycle mutations, making them stable in the open state for prolonged periods of time, a second $594 \mathrm{~nm} \# 1$ light pulse was delivered to close channels. Pulse duration generally ranged from 500 - $1000 \mathrm{~ms}$, but was adapted to the individual channelrhodopsin. Cells were coilluminated with two wavelengths to test the effect of the second wavelength on the open channel state. Therefore, cells were illuminated for $7 \mathrm{~s}$ with the opening wavelengths and during that time, $3 \times 500 \mathrm{~ms}$ light pulses of the wavelength being tested were applied (Figure 2d). To increase the available $594 \mathrm{~nm}$ power, the $940 \mu \mathrm{m}$ light guide was exchanged, first for a $200 \mu \mathrm{m}$ and then finally a $100 \mu \mathrm{m}$ light guide, consequently also decreasing the maximal diameter of the ROE photomask \#1 to $100 \mu \mathrm{m}$ and the system-provided beamsplitter in the DirectFrap module was exchanged for an AHF beam-splitter providing $80 \%$ transmission from the epi-fluorescence and ROE mask illumination and $20 \%$ reflection for the DirectFRAP laser (AHF analysetechnik AG, Tübingen, Germany; cat. no. F21-008).

To test focal optogenetic stimulation and record responses electrophysiologically, $488 \mathrm{~nm}$ light pulses were delivered using DirectFRAP \#6-10, using the 100x objective; for the detection of focal activation using calcium imaging, RCaMP1c wide-field fluorescence was imaged using the 100x objective at $3 \% 561 \mathrm{~nm}$ laser power with $200 \mathrm{~ms}$ exposure times at $2 \mathrm{~Hz}$ and ChR2 L132C was gated with $200 \mathrm{~ms}$ long $488 \mathrm{~nm}$ light pulses using the DirectFRAP \#7 at $2 \%$ laser power. Finally, \#9 \& 10 DirectFRAP photomasks were overlaid with \#15\&16 ROE photomasks to produce a rough macroscopic representation of an illumination inspired by STED/ RESOLFT microscopy (Figure 2e,f).

To investigate the effect of local ChR stimulation on the mobility of BDNF-mRFP puncta, BDNF-mRPF-P2A-hChR2(T159C or L132C)-ECFP expressing cells were imaged using the 63x objective and sequential $0.5 \mathrm{~Hz}, 250 \mathrm{~ms}$ long 405 or $488 \mathrm{~nm}$ light pulses at $20 \%$ laser power ( $300 \mathrm{~W} / \mathrm{cm}^{2}$ or $1,300 \mathrm{~W} / \mathrm{cm}^{2}$ ) were delivered for $1-3 \mathrm{~min}$ using the DirectFRAP photomask \#10 focused onto traversing processes. Before and after the light stimulation puncta were imaged at $0.1 \mathrm{~Hz}$ for $10 \mathrm{or}$ up to $90 \mathrm{~min}$. In order to verify local ChR activation visually red fluorescent genetically encode calcium indicators were imaged at low $561 \mathrm{~nm}$ intensities, while brief $488 \mathrm{~nm}$ laser pulses were delivered through the DirectFRAP photoactivation mask.

For electrophysiological experiments, $\varnothing-25 \mathrm{~mm}$ cultures were transferred to a custom-made imaging chamber, adapted to fit with the motorized stage, in extracellular solution. To single out ChR photocurrents and decrease spontaneous and network activity, $1 \mu \mathrm{M}$ Tetrodotoxin ('TTX', selective sodium channel blocker (Narahashi et al., 1964); Alomone Labs, Jerusalem, Israel; cat. no. T-550), 
$50 \mu \mathrm{M}$ APV (2-amino-5-phosohonopentanoic acid, selective NMDA receptor antagonist (Olverman et al., 1984); Abcam plc, Cambridge, UK; cat. no. ab120003), $10 \mu \mathrm{M}$ CNQX (6-cyano-7-nitroquinoxaline2,3-dione, competitive AMPA/Kainate receptor agonist (Honoré et al., 1987); Sigma; cat. no. C239) and $10 \mu \mathrm{M}$ gabazine (GABA receptor antagonist (Heaulme et al., 1987); Abcam; cat. no. ab120042) was added to the extracellular solution. Patch pipettes were made of fire polished borosilicate capillaries (Harvard Apparatus, Holliston, U.S.A.; cat. no. 300060 , OD $1.5 \mathrm{~mm} \times$ ID $0.86 \mathrm{~mm}$ ) with a horizontal P-97 Micropipette Puller equipped with a $3 \times 3 \mathrm{~mm}$ box filament (Sutter Instruments Co.; cat. no. P-97 \& FB330B). The defined pulling protocol was closest to the ones recommended for patch pipettes provided in the user manuals; and was adapted to produce pipettes with electrical resistances of 3-5M $\mathrm{M}$. Silver wire electrodes were chlorinated using an $\mathrm{ACl}-01$ apparatus (npi electronic, Tamm, Germany; cat. no. ACL-01) and a $2 \mathrm{M} \mathrm{KCl}$ solution. Patch pipettes were backfilled with $7 \mu$ internal solution, using $20 \mu \mathrm{l}$ Microloader pipette tips (Eppendorf; cat. no. 5242 956.003). A pipette holder was mounted on and controlled using a MPC-385-2 micromanipulator system (Sutter Instruments, Inc.). The whole setup was carefully grounded and shielded to reduce electrical noise from nearby components. Whole-cell patch-clamp recordings were obtained with an EPC10 USB double patch-clamp amplifier from HEKA and the corresponding Patchmaster software at $40 \mu \mathrm{s}(25 \mathrm{kHz})$ sampling intervals. Microscope and components including lasers were controlled at first via the Zeiss AxioVision software and later using Zen Blue. Transfected cells were identified by fluorescence and selected for experiments based on health and membrane integrity. Recordings were acquired while voltage-clamping cells at $-70 \mathrm{mV}$ holding potential. Liquid junction potential, pipette and cell capacitance influences were compensated using amplifier-provided internal compensation procedures. Recording files were managed with IGOR Pro (Wavemetrics; version 6.22A), with the addition of the Patcher's Power Tools extension for working with HEKA files, provided by the Department of Membrane Biophysics at the Max-Planck-Institute for Biophysical Chemistry in Göttingen and were arranged for display using Illustrator CS5 (Adobe Systems). 
ROE Mask Illumination for Zeiss Observer

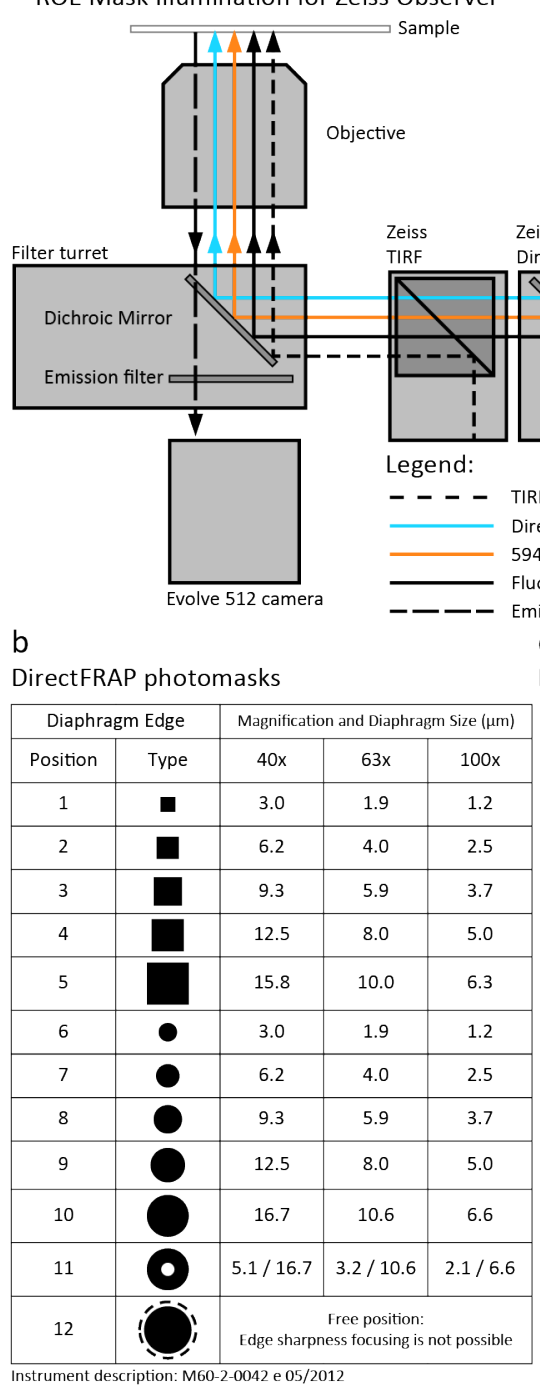

d Whole-Cell Patch-Clamp:

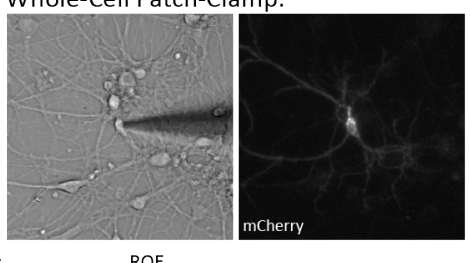

Zeiss $\quad$ ROE e TTL protocols:

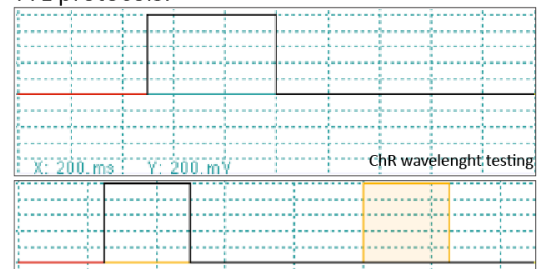

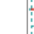

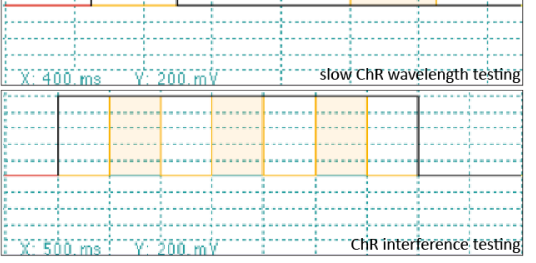

f
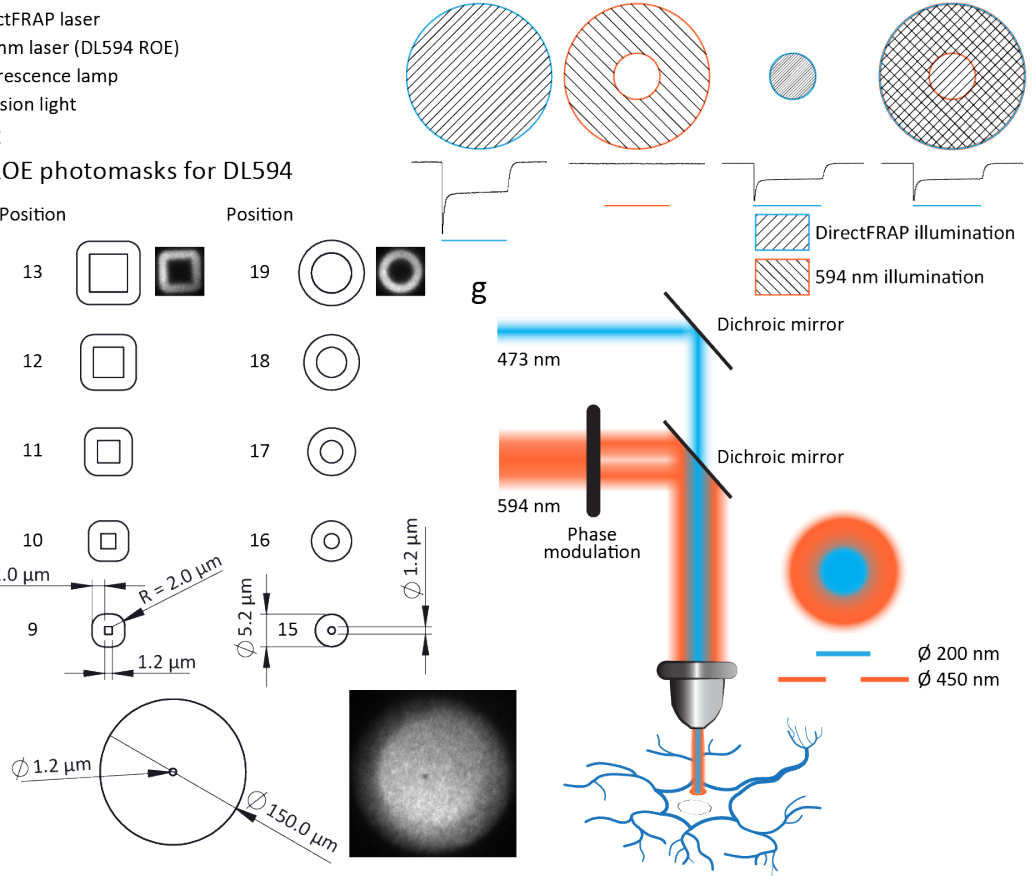

Figure 2: Custom modified microscope light path, photomasks and experimental approach for optogenetic and electrophysiological experiments. (a) Modified schematic from ROE showing light paths and entry for the DL594 on the Axio Observer.Z1. (b) Modified summary of the photoactivation masks of the DirectFRAP system by Zeiss, taken from the instrument description (M60-2-0042 e 05/2012), showing photomask patterns and sizes according to the respective objective magnifications. (c) Specifications for the ROE mask design. Given dimensions correspond to the use of the $100 x$ objective, for the other objectives they increase roughly by a factor of $1.6(63 \mathrm{x})$ and $2.5(40 \mathrm{x})$. Images taken with a 20x objective of a fluorescent probe are shown for the masks in position 1, 13 and 19. (d) Electrophysiological recording of a ChR expressing cell. Expressing cells are identified by fluorescence of the expressed fluorophore fused to ChR. (e) TTL trigger protocols, created within the Pulse Generator of the amplifier software, orchestrating laser pulses. (f) Schematic showing the experimental design and expected electrophysiological readings for the combination of activation with the DirectFRAP photomask and inactivation through the $594 \mathrm{~nm}$ ROE photomask. (g) Ideal experimental outline to reach single synapse area size activation. $473 \mathrm{~nm}$ activation light is brought together with the $594 \mathrm{~nm}$ donutshaped deactivation beam. The two illuminations are overlapping on the outer sides of the activation beam, leaving a depolarized $200 \mathrm{~nm}$ wide spot. 


\section{Results}

\subsection{Nanoscale Optogenetics}

The aim of this project was to investigate the feasibility of optogenetically stimulating highly focal areas on neurons from single synapses down to structures of a few nanometer, by using an illumination strategy borrowed from the field of super resolution microscopy. This strategy consists of illuminating a specific area with a wavelength that activates channelrhodopsins, while simultaneously illuminating an overlapping donut with a wavelength that de-activates these channels, as graphically displayed in Figure $2 f$ and $g$, thereby activating a small central region only. Prospective favorable channelrhodopsin characteristics and candidates were identified and illumination conditions optimized. Finally, we provide data to prove the proposed concept.

\section{Channelrhodopsin characterization}

Due to a lack of sufficient information about the structure-function relationship in channelrhodopsins, it was not possible to predict if any given channel protein can be efficiently closed by illumination with a second wavelength, while being simultaneously illuminated with its opening wavelength. Therefore a substantial and broad range of channelrhodopsin variants had to be tested electrophysiologically for this property. From all tested candidates, the custom-generated hChR2 C128A/H134R/T159C and the slow CoChR variants C108S and C108S/D136A appeared most promising. Their photocurrents could be substantially reduced when gated with $405 \mathrm{~nm}$ light and coilluminated with $594 \mathrm{~nm}$ light to close channels. Also the C1V1 E122T/C167S channel variant showed promising results, but for $561 \mathrm{~nm}$ activation and $405 \mathrm{~nm}$ inactivation. The combination of coexpression of a depolarizing cation-channel and a hyperpolarizing light-gated chloride-pump (eNPAC) also proposes an interesting alternative, in which counterbalancing currents can be used to cause a net voltage change of $0 \mathrm{mV}$ in the area of simultaneous 405 or 488 , and $594 \mathrm{~nm}$ illumination.

As a first step, channels were tested for functional expression in dissociated hippocampal neurons with photocurrents elicited by 405, 488, 561, 594 and $639 \mathrm{~nm}$ single wavelength laser illumination (Figure 3, Figure 4; yellow boxes) and subsequently for their behavior under combined coillumination (Figure 3, Figure 4; blue boxes below respective channels). All available laser combinations for two wavelengths were tested. The hereafter-displayed combinations are those where effects could be observed. The first group of tested channels included the common ChR2 variants with fast-photocycles (H134R, T159C, E123T/T159C and L132C), channels published to have red-shifted activation spectra (namely C1V1 (E122T/E162T) and ReaChr) and channels that were reported to have strong photocurrents (Chronos and CoChr) (Figure 3). Single wavelength responses of the H134R and T159 variants were almost identical. Both demonstrated pronounced peak currents of $0.6-1 \mathrm{nA}$ and stationary currents of around $500 \mathrm{pA}$ for 405 and $488 \mathrm{~nm}$ illumination. These channels only responded weakly or not at all, to wavelengths in the orange and red spectrum. E123T/T159C did exhibit relatively large peak and plateau currents of more than $1.5 \mathrm{nA}$ and $700 \mathrm{pA}$ 
when stimulated with $488 \mathrm{~nm}$, but only weak photocurrents of $250 \mathrm{pA}$ for stimulation with 405,561 and $594 \mathrm{~nm}$. The most intriguing characteristic of this channel is its fast kinetics for which it is popular (Berndt et al., 2011). This characteristic was not specifically assessed, but can be inferred by qualitatively comparing current rise and fall times with other channels. L132C, published to be calcium permeable (Kleinlogel et al., 2011), displayed strong photocurrents of 750 pA for $405 \mathrm{~nm}$ and $1.3 \mathrm{nA}$ for $488 \mathrm{~nm}$ illumination with a significantly reduced peak-stationary current ratio, compared to other channels. Pronounced current rise and especially falling times indicate slow channel kinetics. As anticipated, C1V1 E122T/E162T and ReaChR possess an extended activation spectrum towards the red-shifted wavelengths, but respond almost equally well to 405 and $488 \mathrm{~nm}$. However, photocurrents for both channels ranged around $300-400$ pA. Chronos and CoChR could both be efficiently opened by illumination at 405, 488 and $561 \mathrm{~nm}$. Of these, $488 \mathrm{~nm}$ evoked the strongest photocurrents. With $2 \mathrm{nA}$ to $800 \mathrm{pA}$, Chronos exhibited a very distinct peak-stationary current ratio for $488 \mathrm{~nm}$ stimulation, which was markedly reduced for the other wavelengths tested. CoChR did not show such distinct differences between peak and plateau currents, but featured remarkably strong photocurrents of $3-4$ nA.

Subsequently, combined coillumination experiments were performed to test, if simultaneous illumination with a second wavelength can negatively interfere with the open channel state and thus efficiently close channels. None of the channels from the first group displayed efficient channel closing under simultaneous illumination. Some photocurrent reduction was observed for $488 \mathrm{~nm}$ mediated activation and simultaneous $594 \mathrm{~nm}$ inactivation, however these reductions were very limited. The H134R variant was tested first and displayed some reduction. Decreased activation intensities did not increase the effect further, even though maximum depolarizing photocurrents were reduced. Experiments testing increasing $594 \mathrm{~nm}$ intensities at a constant $488 \mathrm{~nm}$ intensity suggested that photocurrent reduction efficiencies were not yet fully saturated at $100 \% 594 \mathrm{~nm}$, which was the reason for later modifications to the light path of the setup to further increase $594 \mathrm{~nm}$ intensities. T159C demonstrated even weaker photocurrent reduction by $594 \mathrm{~nm}$. Interestingly, reduction in photocurrent was stronger when T159C channels were coilluminated with $405 \mathrm{~nm}$ compared to $594 \mathrm{~nm}$. E123T/T159C and L132C both exhibited a weak photocurrent reduction for the $488 \mathrm{~nm}$ activated state, with simultaneous $594 \mathrm{~nm}$ coillumination, but due to its increased activation properties in the red spectrum, photocurrents of E123T/T159C elicited by $405 \mathrm{~nm}$ light even increased for simultaneous illumination with 561 or $594 \mathrm{~nm}$. The red-shifted channels C1V1 E122/E162T and ReaChR were mainly tested for $405 \mathrm{~nm}$ mediated photocurrent reduction, because simultaneous illumination with 488,561 or $594 \mathrm{~nm}$ light in addition to 405 or $488 \mathrm{~nm}$ activated currents caused a further photocurrent increase. C1V1 and ReaChR channels exhibited only mild $405 \mathrm{~nm}$ mediated photocurrent reduction when activated with 561 or $488 \mathrm{~nm}$. Interestingly, CoChR photocurrents could be reduced when 488 and $561 \mathrm{~nm}$ activation was combined with $405 \mathrm{~nm}$ illumination as well. This was particularly surprising in the case of $561 \mathrm{~nm}$ evoked photocurrents, because the tests with single wavelengths had revealed, that $405 \mathrm{~nm}$ evoked currents were stronger 
than those evoked by $561 \mathrm{~nm}$. Thus we expected a further photocurrent increase with coillumination. $594 \mathrm{~nm}$ had no noticeable effect on the different conducting states, but $561 \mathrm{~nm}$ coillumination decreased currents evoked by $405 \mathrm{~nm}$. Chronos did not show any significant photocurrent reduction under the tested conditions, only very weakly for simultaneous $405 \mathrm{~nm}$ illumination, when activated with $488 \mathrm{~nm}$. On the contrary and as expected from the single wavelength results; 405,488 or $561 \mathrm{~nm}$ coillumination did increase photocurrents of the 405 or $561 \mathrm{~nm}$ activated states.

Based on published information about their closing behavior, the above mentioned group of channels was extended to include step-function opsin variants, including the 'classical' stable stepfunction ChR2 C128S/D156A also termed SSFO, the custom-combined variants C128A/H134R/T159C and C128A/L132C/T159C, a C1V1-derived E122T/C167S and the CoChR-based C108S and C108S/D136A variants. Chronos slow photocycle variants were also tested, but did not express well in dissociated hippocampal neurons. All of these channels are best characterized by their much slower photocycles or more precisely, stable open channel state. In addition we also included eNPAC, a co-expression construct, combining co-expression of halorhodopsin 3.0 and ChR2 H134R (Figure 4). Channels with a stable open state have in common that they are very light sensitive. Even under unspecific broad-spectrum daylight conditions channels open and start accumulating in the conducting open channel state. Therefore channels were closed before and after each experiment by a brief illumination with the respective closing wavelength. The classical stable step-function opsin, C128S/D156A, was tested first. Generally, it can be noted, that the mutations giving rise to a slow photocycle phenotype significantly reduce photocurrents; these two variables appear to be directly coupled. If $\mathrm{C} 128$ and D156 or their corresponding sites in other channels are mutated to the slowphotocycle variants, a drop in the photocurrent of around $90 \%$ can be expected. For ChR2 C128S/D156A, 405 and $488 \mathrm{~nm}$-evoked photocurrents ranged around $30-70 \mathrm{pA}$. Based on information in the relevant literature and our experiences with the SSFO channel and the group of channels with fast photocycles, we created the two custom ChR2 variants C128A/H134R/T159C and C128A/L132C/T159C, hoping to combine C128A switching properties and step function like behavior (Berndt et al., 2009; Prakash et al., 2012) with the stable and strong photocurrents of the H134R, T159C or L132C variants. Both channels did exhibit photocurrent responses of $350-400$ pA for 405 or $488 \mathrm{~nm}$ illumination and some weak photocurrents for $561 \mathrm{~nm}$. For both stimulation wavelengths, currents directly rose to stationary levels and without any detectable peak photocurrents. When $488 \mathrm{~nm}$ exposure was stopped, currents exhibited a small increase, because even $488 \mathrm{~nm}$ light partially closes channels, an effect that was not detected for $405 \mathrm{~nm}$ stimulation. The major difference between these channels was the marked slower channel kinetics of the L132C containing variant. Even when illuminated with only $594 \mathrm{~nm}$ at full intensity, photocurrents decreased much slower than for other channels and exhibited a declining curve, suggesting a minimum of two separate components, an initial fast component and a second much slower component. Similar results were obtained for the mutated CoChR variants C108S and C108S/D136A. However, $405 \mathrm{~nm}$ - 
mediated currents were significantly lower than currents evoked by $488 \mathrm{~nm}$ for these channels, and like the SSFO double mutant, mutated CoChR variants maintained only a fraction of the high currents from the original channels without the step-function mutation. Photocurrents were reduced to a mere 50 - 300 pA for 405 and $488 \mathrm{~nm}$ illumination. No marked responses to red-shifted wavelengths were detected for the stimulation intensities and durations that were tested. The CoChR C108S/D136A mutant also exhibited slightly slower closing kinetics. In contrast to the double mutant, the single $\mathrm{C} 108 \mathrm{~S}$ mutant exhibited $488 \mathrm{~nm}$ mediated channel closure, as seen from the photocurrent increase after stopping illumination. This effect was markedly reduced for the double mutant and not at all present when stimulated with $405 \mathrm{~nm}$. C1V1 E122T/C167S is the corresponding step function version of the C1V1 channel. In contrast to the five other step function opsins mentioned above and unlike the original C1V1 channel this version was derived from, C1V1 E122T/C167S did not exhibit $405 \mathrm{~nm}$ evoked photocurrents, but continued to respond to $488,561,594$ and weakly also to $639 \mathrm{~nm}$. Instead, the channel containing the step function mutations could now be closed by exposure to $405 \mathrm{~nm}$. $488 \mathrm{~nm}$ illumination evoked typical slightly increased initial photocurrents, subsequently reducing to a stable plateau; while 561 and $594 \mathrm{~nm}$ excitation evoked more step-like photocurrents.

As opposed to other approaches, the use of the eNPAC construct does not depend on a specific illumination to close channels, but rather on a balance between blue light activation of ChR2 H134R to depolarize cells and red light activation of NpHR3.0 to hyperpolarize cells. Photocurrent responses were detected for all tested wavelengths. 405 and $488 \mathrm{~nm}$ evoked responses were almost identical to standard ChR2 H134R responses. However, photocurrents for both wavelengths and especially for $488 \mathrm{~nm}$ were slightly weaker and displayed a small and brief transient just after the illumination was stopped. NpHR3.0 is not exclusively active at red wavelengths, but is also weakly activated by $488 \mathrm{~nm}$ and less by $405 \mathrm{~nm}$ illumination. For that reason, the $488 \mathrm{~nm}$ currents are more affected by hyperpolarizing currents than currents evoked by $405 \mathrm{~nm}$. The small current increase after the stimulation originates in part from the same phenomenon. Because the pumping of chloride-ions by NpHR3.0 is an active process driven by light, it stops almost instantaneously when the illumination is discontinued. ChR2 H134R channels passively conduct ions along their concentration gradients as long as their conducting pore is open. The channels open when they are activated with light and change their conformation, passing through several intermediate states, until they finally reach their closed ground state again. This process takes time, represented by the curve of the photocurrent when illumination is stopped. For a brief moment, the hyperpolarizing current drops faster than the depolarizing currents and therefore causes further depolarization. This effect is not present for $405 \mathrm{~nm}$ excitation, implying that halorhodopsins are not significantly activated at this wavelength and the drop in the hyperpolarizing current does not surpass the decrease in depolarization. When illuminated with 561 and $594 \mathrm{~nm}$, hyperpolarizing currents of around 400 pA were recorded from eNPAC-expressing cells. Because the ChR2 H134R does not have significant red wavelength activity, these currents are predominantly from NpHR3.0 activation. 
We then tested various channels for their inactivation properties during simultaneous illumination with 405 or $488 \mathrm{~nm}$ light, and with 561 or $594 \mathrm{~nm}$ light. The C128S/D156A channel displayed only weak photo-inactivation for exposure to $561 \mathrm{~nm}$ and no effect of $594 \mathrm{~nm}$ coillumination. Because this channels also had weak photocurrents in addition, it was not interesting for our purpose and was not investigated further. The C128A/H134R/T159C and C128A/L132C/T159C variants exhibited slightly stronger photocurrent reduction under 488 and $594 \mathrm{~nm}$ coillumination and markedly stronger photocurrent reduction for 405 and $594 \mathrm{~nm}$ coillumination. The channel closing speed depended on the $594 \mathrm{~nm}$ light intensity and was not fully saturated, even with maximal intensity in our experiments. The very slow closing kinetics of the L132C containing variant rendered this variant suboptimal. For CoChR properties changed more profoundly. While the original version did not possess any $594 \mathrm{~nm}$ mediated photocurrent inactivation, the two mutated step-function variants exhibited some inactivation with $594 \mathrm{~nm}$ light when activated with $488 \mathrm{~nm}$ and more when activated with $405 \mathrm{~nm}$. This effect appeared more promising for the double mutant, because $405 \mathrm{~nm}$ stimulated currents were greater. The photocurrent reduction observed with activation 488 and $405 \mathrm{~nm}$ coillumination of the original channel was not noticeable in the two mutant channels. As expected, the C1V1 E122T/C167S showed superb photocurrent reduction when stimulated with $561 \mathrm{~nm}$ and coilluminated with $405 \mathrm{~nm}$ (Prigge et al., 2012). High $405 \mathrm{~nm}$ intensities were more important than low $561 \mathrm{~nm}$ intensities for efficient photocurrent reduction. In initial experiments a photocurrent reduction of about $84 \%$ could be achieved. However, $405 \mathrm{~nm}$ illumination alone already evoked currents of $5.1 \%$ of the maximal photocurrents evoked by $561 \mathrm{~nm}$. This current however cannot be eliminated by illumination with another wavelength, but dissipated over time with the channels closing. It is interesting to note that $488 \mathrm{~nm}$ coillumination also reduced $561 \mathrm{~nm}$ evoked photocurrents, even though currents evoked by $488 \mathrm{~nm}$ illumination alone are usually stronger than $561 \mathrm{~nm}$ currents. And in contrast to the inactivating influence of $405 \mathrm{~nm}$ light on $561 \mathrm{~nm}$ evoked currents, $405 \mathrm{~nm}$ coillumination together with $488 \mathrm{~nm}$ appeared to increase photocurrents further. In neurons transfected with eNPAC, illumination with wavelengths activating ChR2 H134R or NpHR3.0 evoked the corresponding depolarizing or hyperpolarizing currents. Similar hyperpolarizing photocurrents were evoked by 561 or $594 \mathrm{~nm}$ stimulation of NpHR3.0. Using $405 \mathrm{~nm}$ coillumination caused only weak reduction of the depolarizing current, because NpHR3.0 is only weakly activated at this wavelength. 


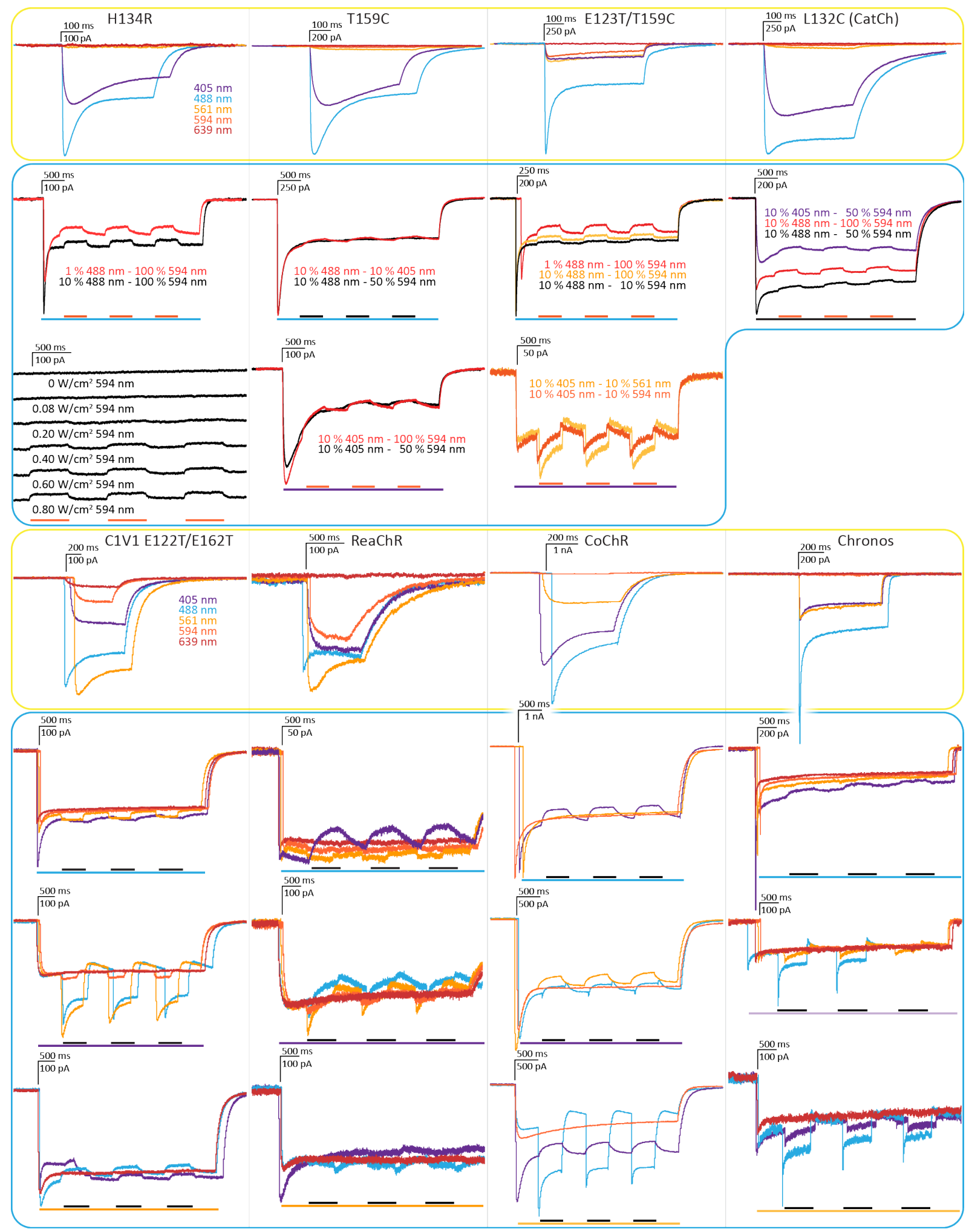

Figure 3: Illumination evoked responses of light-gated channels with fast photocycles. Photocurrents elicited by 405, 488, 561, 594 and $639 \mathrm{~nm}$ single wavelength laser illumination (yellow boxes) and responses to simultaneous illumination (blue boxes below respective channels). Photocurrent recordings at specific illumination wavelengths are indicated by the respective color of the recording. Scale bars above individual experiments indicate recorded currents (vertical) and time (horizontal). For coillumination experiments bars below recordings indicate time of illumination for the indicated wavelength. Black bars refer to coillumination with the color of the shown traces. Activating laser intensities were $10 \%\left(405 \mathrm{~nm} 86.83 \mathrm{~mW} / \mathrm{cm}^{2} ; 488 \mathrm{~nm}\right.$

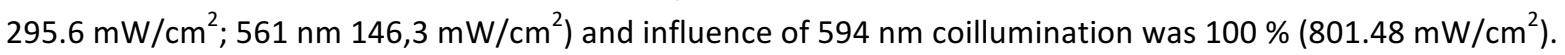




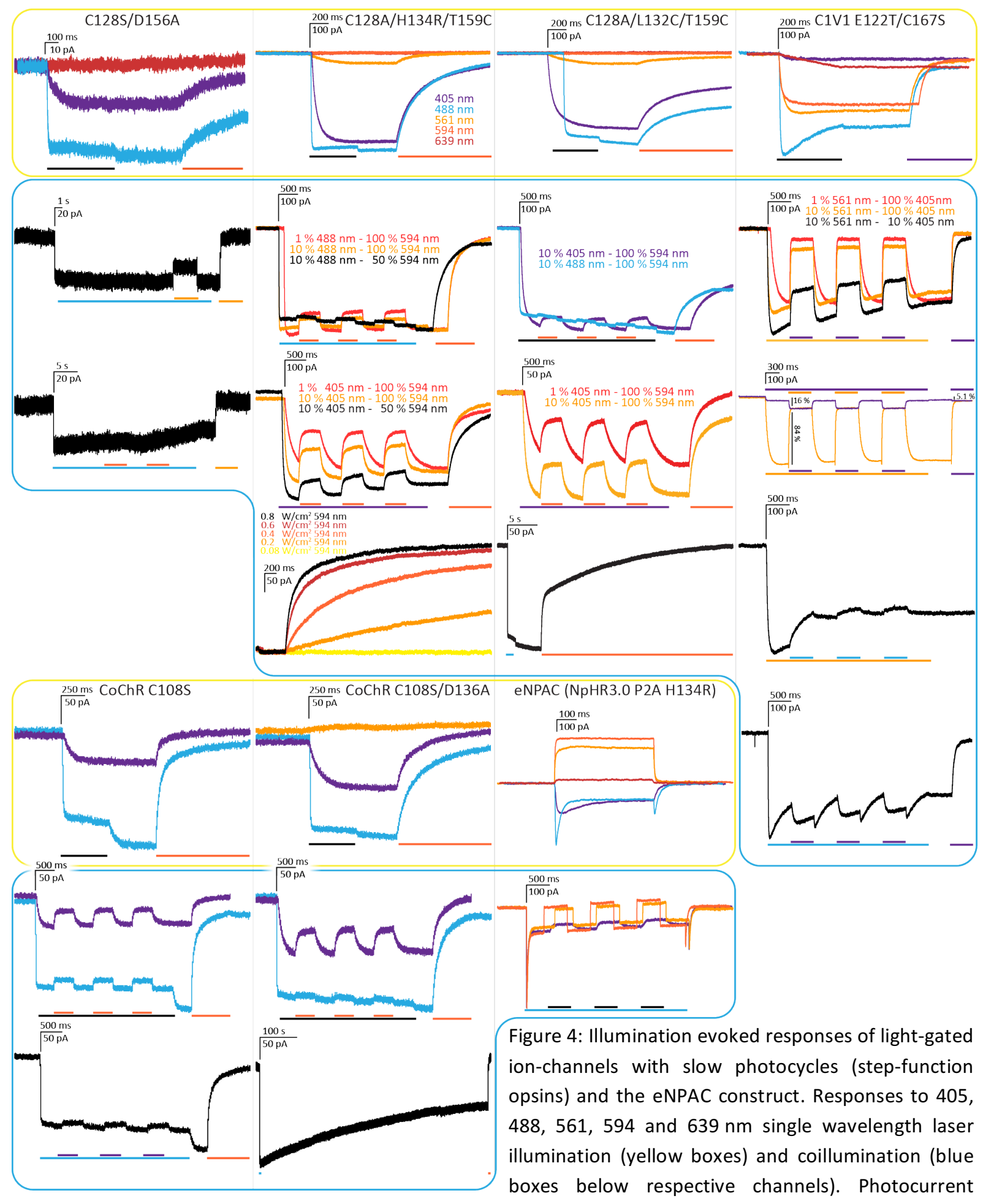

recordings at specific wavelength illumination are indicated by the respective color of the recording. Scale bars above individual experiments indicate current (vertical) and time (horizontal). For coillumination experiments bars below the recordings indicate wavelengths and timing. Black bars refer to the illumination time of each trace shown in color. Unless otherwise indicated, activating laser intensities were $10 \%(405 \mathrm{~nm}$

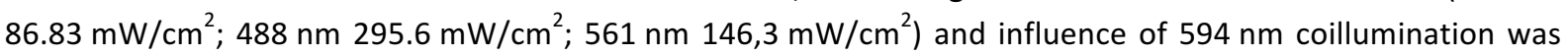
$100 \%\left(801.48 \mathrm{~mW} / \mathrm{cm}^{2}\right)$. 


\section{Efficient photocurrent reduction by intensified $594 \mathrm{~nm}$ illumination}

During testing it became apparent that even when the $594 \mathrm{~nm}$ laser was used at $100 \%$ $\left(801.48 \mathrm{~mW} / \mathrm{cm}^{2}\right)$, maximal channel inactivation was not reached. Therefore we increased the available $594 \mathrm{~nm}$ laser intensity further by modifying key parts of the light path and exchanged the light guide from the DL594 laser module to the microscope with the beam splitter in the DirectFRAP unit (see 'Materials and Methods' section for further detail) and tested the effect of this modification on the most promising candidates, ChR2 C128A/H134R/T159C (Figure 5 left and middle graphics) and the CoChR mutant variants C108S and C108S/D136 (Figure 6). Channels were activated with $10 \%$ laser power of $488\left(41.92 \mathrm{~W} / \mathrm{cm}^{2}\right)$ or $20 \% 405 \mathrm{~nm}\left(9.92 \mathrm{~W} / \mathrm{cm}^{2}\right)$ illumination through the DirecFRAP laser path (Figure 2a) and inactivation was probed by simultaneously illuminating cells three consecutive times with $594 \mathrm{~nm}$ light for $500 \mathrm{~ms}$ each. The photocurrent values from these three exposures were pooled and compared to the average maximum photocurrent determined from the $500 \mathrm{~ms}$ between coillumination and a final $594 \mathrm{~nm}$ exposure. Additionally, activation intensities from 1 to $20 \%$ were tested against $100 \%$ DL594 power (129.75 W/ $\mathrm{cm}^{2}$ ) (Figure 5 - graphics on right). Using the modified setup, we were able to reduce depolarizing currents of ChR2 C128A/H134R/T159C elicited with $488 \mathrm{~nm}$ by $54 \%$. If, however, channels were exposed to $405 \mathrm{~nm}$ activation light, photocurrent inactivation of up to $90 \%$ was possible with marginal differences in the depolarizing current strength, compared to $488 \mathrm{~nm}$. Nevertheless, both experiments indicated that with the current equipment a complete reduction of $100 \%$ is not feasible. Different activation intensities increased photocurrents only minimally for $488 \mathrm{~nm}$ activation, but significantly affected photocurrent reduction. For $405 \mathrm{~nm}$ this was opposite, increases of $405 \mathrm{~nm}$ power up to $20 \%$ significantly increased depolarizing currents, maintaining efficient photocurrent reduction.
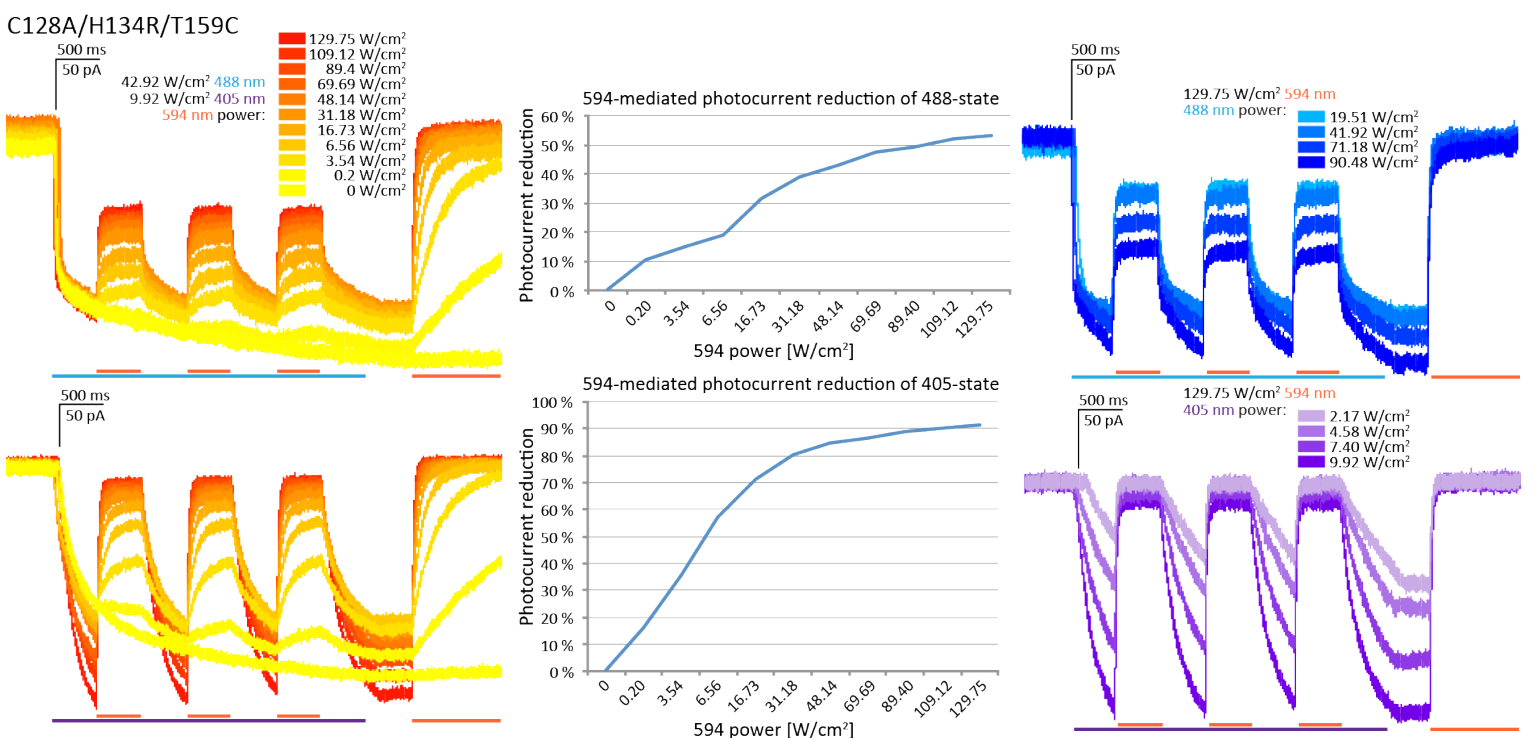

Figure 5: Reduction of 488 and $405 \mathrm{~nm}$ evoked photocurrents in ChR2 C128A/H134R/T159C. Left two graphs depict the effect of different $594 \mathrm{~nm}$ laser powers on $10 \% 488 \mathrm{~nm}$ or $20 \% 405 \mathrm{~nm}$ evoked currents; $594 \mathrm{~nm}$ laser power as indicated in the figure legend, was tested in $10 \%$ steps from 0 to $100 \%$. Middle diagrams represent the respective relationship between $594 \mathrm{~nm}$ laser intensity and photocurrent reduction. Right two graphs depict the influence of the activating light intensity $(1,5,10$ and $20 \%)$ on $594 \mathrm{~nm}$ mediated photocurrent reduction. Scale bars indicate recorded currents (vertical) and time (horizontal). 
Tests for 488 and $405 \mathrm{~nm}$ activation of the CoChR mutant variants yielded comparable results (Figure 6). Photocurrent reduction was most efficient for 405 and $594 \mathrm{~nm}$ coillumination. There was no significant advantage in using $488 \mathrm{~nm}$ or higher intensities. A photocurrent increase when $488 \mathrm{~nm}$ illumination is discontinued was also visible for the CoChR channel, especially for the C108S mutant. In general, currents of the CoChR step function variants were weaker than the ChR2 step function variants and did not increase when higher $405 \mathrm{~nm}$ intensities were used. However, $405 \mathrm{~nm}$ evoked currents could be efficiently reduced by exposure to $594 \mathrm{~nm}$. These experiments suggest $405 \mathrm{~nm}$ as the ideal activation wavelength for experiments where strong photocurrents and efficient photocurrent reduction, using $594 \mathrm{~nm}$ coillumination, is needed.

Effects of strong 594 laser power on fastphotocycle variants

We also tested whether higher $594 \mathrm{~nm}$ intensities reduce 488 and $405 \mathrm{~nm}$ evoked photocurrents of the fast-photocycle variants ChR2 T159C and CoChR more efficiently (Figure 7). However, illumination at $594 \mathrm{~nm}$ at high intensity alone, without coillumination, evoked photocurrents of $100-200$ pA in cells expressing either one of these channels. No significant photocurrent reduction during coillumination with 405 or $488 \mathrm{~nm}$ light for either of these channels was seen. Instead small photocurrent transients could be observed, which were most pronounced for 405 and $594 \mathrm{~nm}$ coillumination of CoChR expressing cells. This is quite critical, because it implies that slow-photocycle variants have a significant conductance at $594 \mathrm{~nm}$, which due to the prolonged open channel states is not detected, but which may cause a portion of channels to remain open when illuminated at $594 \mathrm{~nm}$.

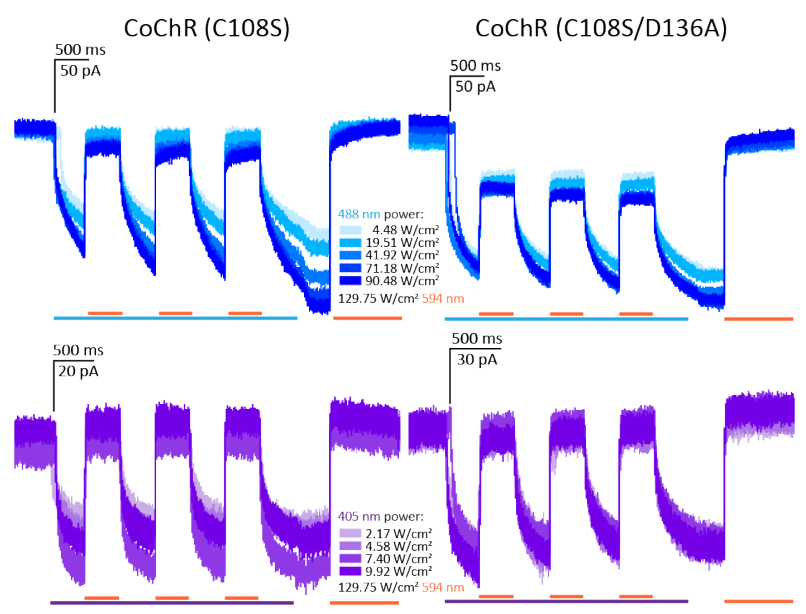

Figure 6: Photocurrent reduction of 488 and $405 \mathrm{~nm}$ evoked currents in CoChR C108S and C108S/D136A mustants. Trace and stimulation bar colors represent individual laser wavelengths and intensities $(5,10,15$, $20 \%)$.

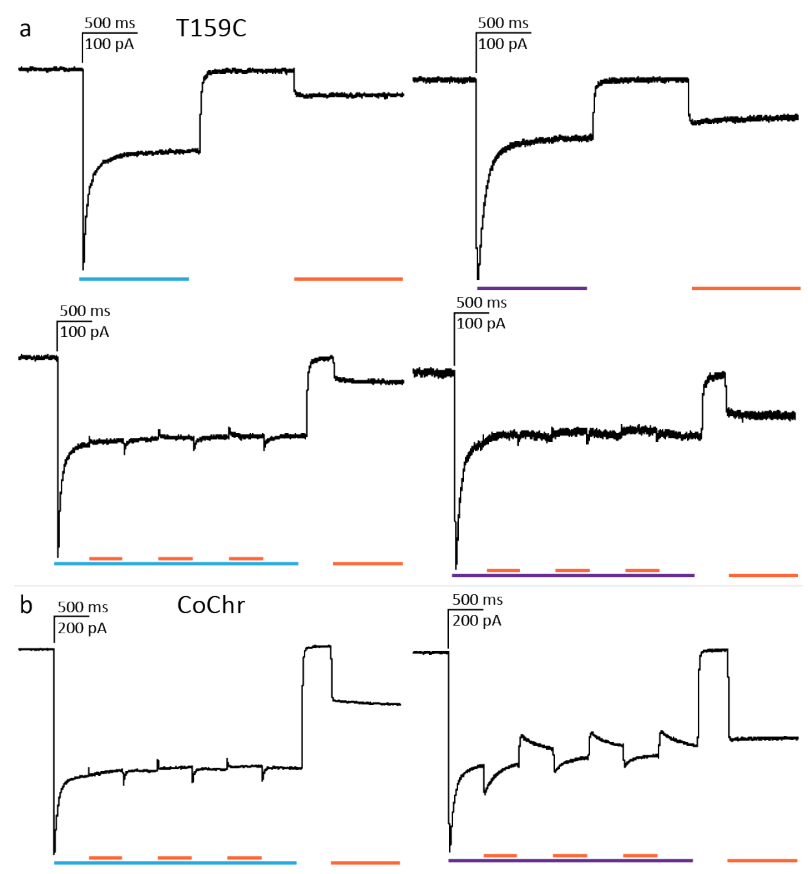

Figure 7: Effect of higher $594 \mathrm{~nm}$ laser intensity on fast photocycle variants. Photocurrents evoked by $10 \%$ $488 \mathrm{~nm}$ or $20 \% 405 \mathrm{~nm} 16.7 \mu \mathrm{m}$ spotlight stimulation, using the DirectFRAP system with coillumination of 594 $\mathrm{nm}$ light for T159C (a) and CoChR (b) channels. Stimulation onset, photocurrents and time are indicated by scale bars. 


\section{Focal optogenetical stimulation of neuronal processes}

Based on the experience, gained from the previous experiments, we tested focal activation of proximal neuronal processes of cells expressing the fast photocycle ChR2 T159C channel with $488 \mathrm{~nm}$ light from the DirectFrap unit. Photocurrents elicited by stimulations of membrane areas with diameters down to $1.2 \mu \mathrm{m}$ could reliably be detected using electrophysiological patch-clamp recordings in the whole-cell configuration (Figure 8a). However, detectable photocurrents were already weak, implying that photocurrents from smaller areas will be beyond detection limits. Photocurrents from focal activation would be expected to be even smaller for the slowphotocycle channel variants, which have significantly reduced photocurrents.

To visually confirm the site of stimulation, we tested genetically encoded calcium indicators. Due to spectral overlap with channel activation, most commonly used indicators, i.e. GCaMPs are activated by blue light, could only be imaged at wavelengths

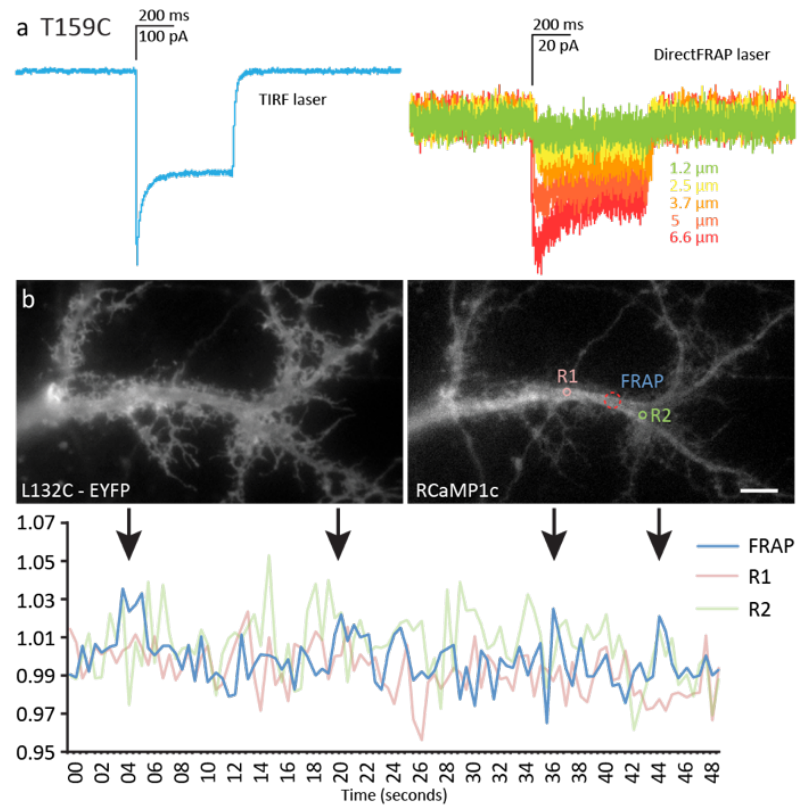

Figure 8: Electrophysiological recordings and visual detection of focally stimulated areas. (a) T159C channelrhodopsin photocurrents elicited by $20 \%$ $488 \mathrm{~nm}$ TIRF or DirectFRAP (photoactivation masks $\# 6$ - 10) laser stimulation of the cell body. (b, c) Focal $488 \mathrm{~nm}$ activation of ChR2 L132C using the DirectFRAP mask \#7 $(2.5 \mu \mathrm{m})$ on a neuronal process visualized by RCaMP1c fluorescence. (b) Images show L132C-EYFP (left) and RCaMP1c (right) fluorescence signal within the area of stimulation (FRAP) and two nearby control areas (R1 and R2). Scale bar is $5 \mu \mathrm{m}$. (c) RCaMP1c signal fluctuations in the 3 ROls. Arrows at 4, 20, 37 and 45 seconds indicate delivery of DirectFRAP laser pulses.

which depolarized cells, making it impossible to use them for the visualization of local $\mathrm{Ca}^{2+}$ concentration increases. RCaMP1c however, can be imaged at a low $561 \mathrm{~nm}$ intensities and therefore be combined with most blue-light activated channels. RCaMP1C and the ChR2 L132C variant were expressed in neurons and cells briefly stimulated with $2.5 \mu \mathrm{m}$ spotlight $488 \mathrm{~nm}$ light, using the DirecFRAP system, while imaging RcaMP1c calcium indicator fluorescence at $561 \mathrm{~nm}$ (Figure 8b). Interestingly, small increases in RCaMP1c fluorescent signal were detected reliably during photostimulation. These signal increases were however very small and difficult to distinguish from background noise of the two control areas (R1 and R2) in single trials. Most other channel variants do not possess calcium-ion permeability. Their detection will therefore mainly depend on calcium concentration increases through the opening of nearby voltage-gated calcium channels. This may further decrease the resolution for visual confirmation of the activated area, especially at lower diameters. 


\section{Dual wavelength illumination for focal depolarization on the macro scale}

To verify the general concept and feasibility of focal channel activation for areas at and beyond the diffraction limit of light, we performed validation experiments at a larger scale. To accomplish this, we used photoactivation masks for 405 or $488 \mathrm{~nm}$ illumination of 6.2 and $16.7 \mu \mathrm{m}$ wide areas in combination with a donut-shaped photoactivation mask (Figure 9a) to stimulate neurons expressing the eNPAC (Figure 9b) or C128A/H134R/T159C channels (Figure 9c). When eNPAC expressing cells were stimulated with 6.2 or $16.7 \mu \mathrm{m}$ spots with $488 \mathrm{~nm}$ laser light, they responded with 20 or $50 \mathrm{pA}$ photocurrents, respectively. When $16.7 \mu \mathrm{m} 488 \mathrm{~nm}$ stimulation of ChR2 was combined with $594 \mathrm{~nm}$ costimulation of halorhodopsins, using an overlapping donut-shaped photoactivation mask at different $594 \mathrm{~nm}$ light intensities (resulting in an activated area of approximately $6.2 \mu \mathrm{m}$ ), net photocurrents were reduced to values lower than (Figure 9b - red traces) or equivalent to (Figure $9 \mathrm{c}$ - black traces) depolarization achieved with the $6.2 \mu \mathrm{m}$ stimulation mask alone. This demonstrates that halorhodopsin currents are tunable in their strength by adjusting the $594 \mathrm{~nm}$ laser power, which can be set to counterbalance the ChR2 mediated depolarization in the coilluminated area. The use of $405 \mathrm{~nm}$ laser light for stimulation, instead of $488 \mathrm{~nm}$, would increase ChR2 photocurrent strength and remove the brief photocurrent transients observed following illumination.
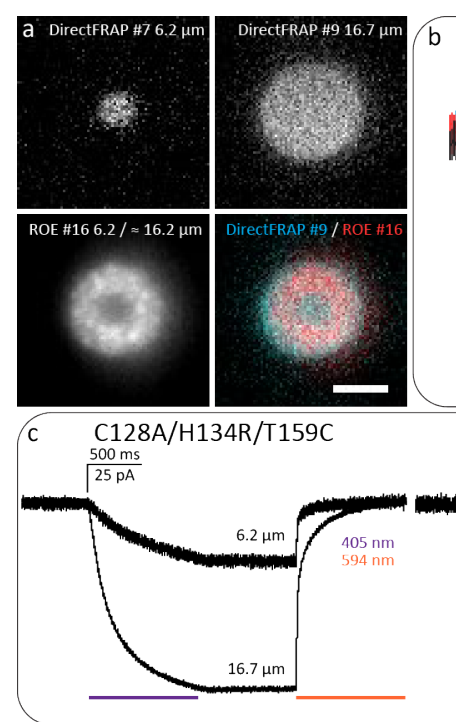
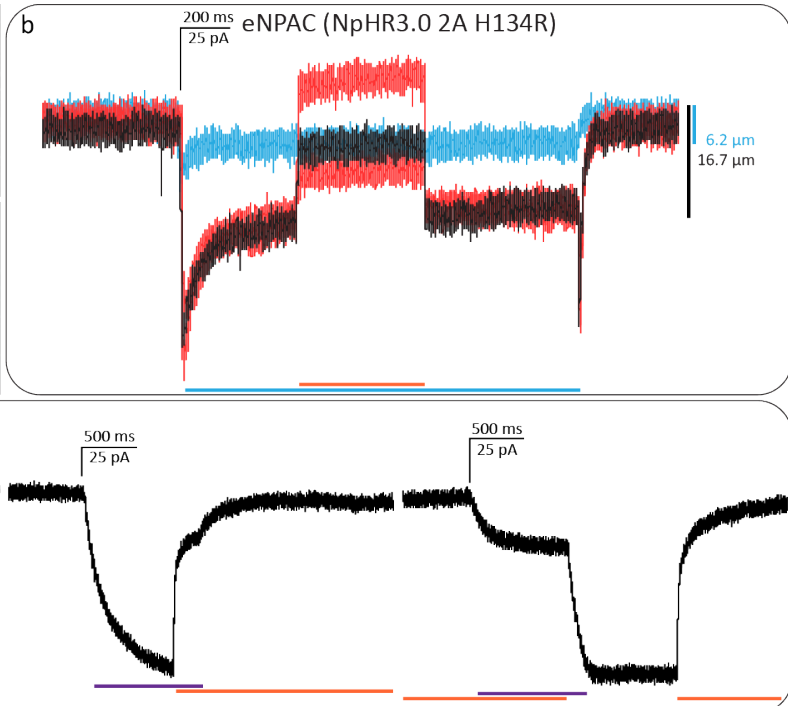

Figure 9:

Focal stimulation proof of concept at the macro scale. (a) Images of yellow text-marker, used as a dye for a uniform fluorescent field (for $488 \mathrm{~nm}$ ) and Rhodamine B (594 $\mathrm{nm})$, excited using 488 $\mathrm{nm}$ DirectFRAP \#7 and 9 and $594 \mathrm{~nm}$ ROE \#16 photomask illumination with a 40x objective. Lower-right image shows a two-color merge of

DirectFRAP \#6 and ROE \#16 photomask images. A substantial part of the $488 \mathrm{~nm}$ illuminated is overlapped by $594 \mathrm{~nm}$ illumination, leaving a center spot of roughly $6.2 \mu \mathrm{m}$ that is not coilluminated. Scale bar is $10 \mu \mathrm{m}$. (b) eNPAC approach using counterbalancing currents. Traces represent photocurrents elicited by DirectFRAP illuminations with 6.2 (blue trace) or $16.7 \mu \mathrm{m}$ (black trace) diameter; $16.7 \mu \mathrm{m}$ photostimulation was combined with a $500 \mathrm{~ms}$ pulse from the DL594 laser using the ROE photomask \#16. $594 \mathrm{~nm}$ laser intensity can be tuned to drive halorhodopsin-mediated hyperpolarizing currents to be weaker, stronger (red trace) or equivalent (black trace) to current elicited by $6.2 \mu \mathrm{m}$ stimulation of ChR2. (c) Photocurrents of the ChR2 C128S/H134R/T159C variant evoked by 6.2 or $16.7 \mu \mathrm{m} 20 \% 405 \mathrm{~nm}$ laser stimulation and subsequent $594 \mathrm{~nm}$ illumination mediated closure (left). When DirectFRAP photomask stimulation was combined with $594 \mathrm{~nm}$ ROE photomask \#16 coillumination (middle and right), the photocurrent can be reduced to levels close to those evoked by $6.2 \mu \mathrm{m}$ stimulation.

In a second approach the same illumination principle was applied to the ChR2 slow-photocyle variant C128A/H134R/T159C (Figure 9c). The respective evoked photocurrents for 6.2 and $16.7 \mu \mathrm{m}$ wide 
areas, illuminated with $405 \mathrm{~nm}$ light, were 50 and $150 \mathrm{pA}$, respectively. If $16.7 \mu \mathrm{m} 405 \mathrm{~nm}$ photostimulation was combined with an overlapping donut of $594 \mathrm{~nm}$ illumination, photocurrents could be reduced to values close to those evoked by stimulation of a $6.2 \mu \mathrm{m}$ area alone. This was independent of the order in which the different wavelengths were applied.

\section{Different illumination approaches for reaching focal photostimulation in the nanoscale}

Based on our experiments, there are four illumination approaches using light-gated ionchannel/pumps, that could be used to achieve highly focal photostimulation (Figure 10). The first approach is simultaneous activating and inactivating wavelength illumination (Figure 9c). For stepfunction opsin channels that do not show sufficient inactivation during coillumination, sequential illumination could be performed. In this second approach, channels are opened with one wavelength and then subsequently closed in an outer donut region, leaving a highly focal area in the center that depolarizes. Subsequently, the remaining open channels can be closed by illumination of the whole field. For this approach the time between initial channel opening and closing of the outer channels should be optimized to be as short as possible. The third option makes use of the eNPAC construct and the possibility to control depolarizing and hyperpolarizing currents with different wavelength illuminations. Unlike for the two other approaches, here the area with activated channels would remain constant, but a second channel type, i.e. halorhodopsins or other hyperpolarizing ionchannels/pumps can be used to counterbalance the depolarizing currents in the area where no depolarization is wanted. Theoretically this approach can also be inverted, producing highly focal areas of hyperpolarization in a fourth approach.

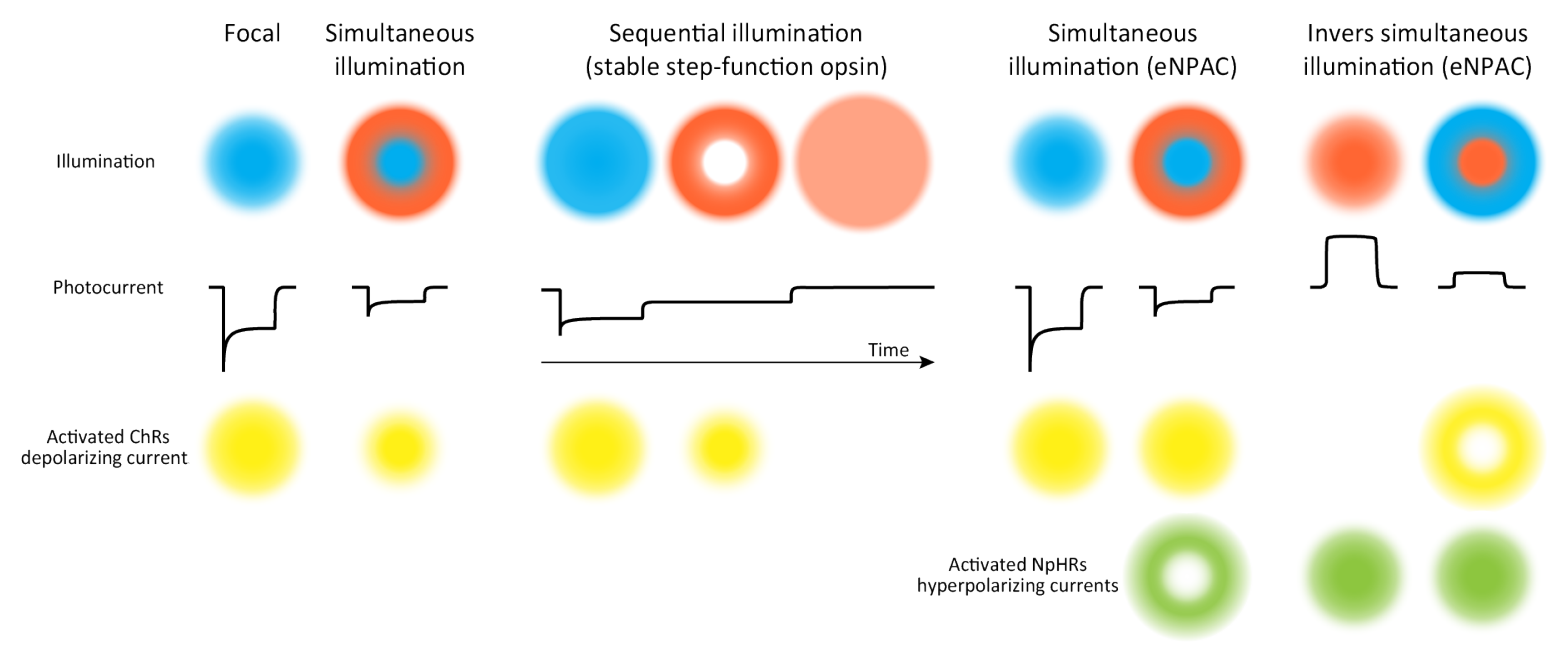

Figure 10: Illumination approaches for focal photostimulation using light-gated ion-channels/pumps.

Schematics summarize four general approaches. 1) Focal stimulation using a DirectFRAP-like photostimulation. Simultaneous illumination with an overlapping donut of the appropriate inactivating wavelength, reduces the area of depolarization. 2) Step-function opsins could be sequentially illuminated: channel opening can be followed instantaneously by closure of channels leaving a focal area of open channels. Remaining open channels can be closed by whole field illumination. 3) Hyperpolarizing currents can be used to counterbalance depolarizing currents in a coilluminated area. 4) This approach can also be inverted, creating a focal area of hyperpolarization. 


\subsection{BDNF-mRFP1 migration and cell-to-cell transfer}

\section{Effects of focal optogenetic stimulation on BDNF trafficking}

The focal activation approaches described above allow us to test the hypothesis that BDNF vesicles are recruited specifically to active synapses/sites in neuronal processes, potentially to modulate the function of these synapses. Fluorescently-tagged BDNF is commonly used as a marker of BDNF trafficking. When overexpressed in neurons and imaged using epifluorescence microscopy, BDNF-GFP puncta appear highly mobile and can be found actively perambulating processes (Figure 11a,b), as already demonstrated elsewhere (Dean et al., 2012; Hartmann et al., 2001). Co-expression of ChR2 under CaMKII promoter control with BDNF-mRFP1, in BDNF-mRFP1-P2A-ChR2-ECFP transfected cells, results in depolarizing photocurrents in response to 405 and $488 \mathrm{~nm}$ light exposure (Figure 11c). Currents were not detected in cells expressing only BDNF-mRFP1-P2A-ECFP. Interestingly, when these cells were photostimulated with $405 \mathrm{~nm}$ light for $3 \mathrm{~min}$ using the DirectFRAP system, we observed accumulations of fluorescent BDNF-mRFP1 puncta over the course of $90 \mathrm{~min}$; subsequently the accumulations gradually disappeared (Figure 11d). During this time fluorescent puncta were also observed traversing through this area of accumulation. These results however have to be interpreted carefully. Although occurring less frequently and with longer and more intense stimulation protocols, similar accumulations were found in control experiments with only the ECFP fluorophore instead of the ChR2, indicating that the observed accumulation could be the result of $405 \mathrm{~nm}$ illumination. Membrane integrity was assessed and did not appear ruptured or otherwise affected. $488 \mathrm{~nm}$ illumination was not used because of possible mRFP1 bleaching.
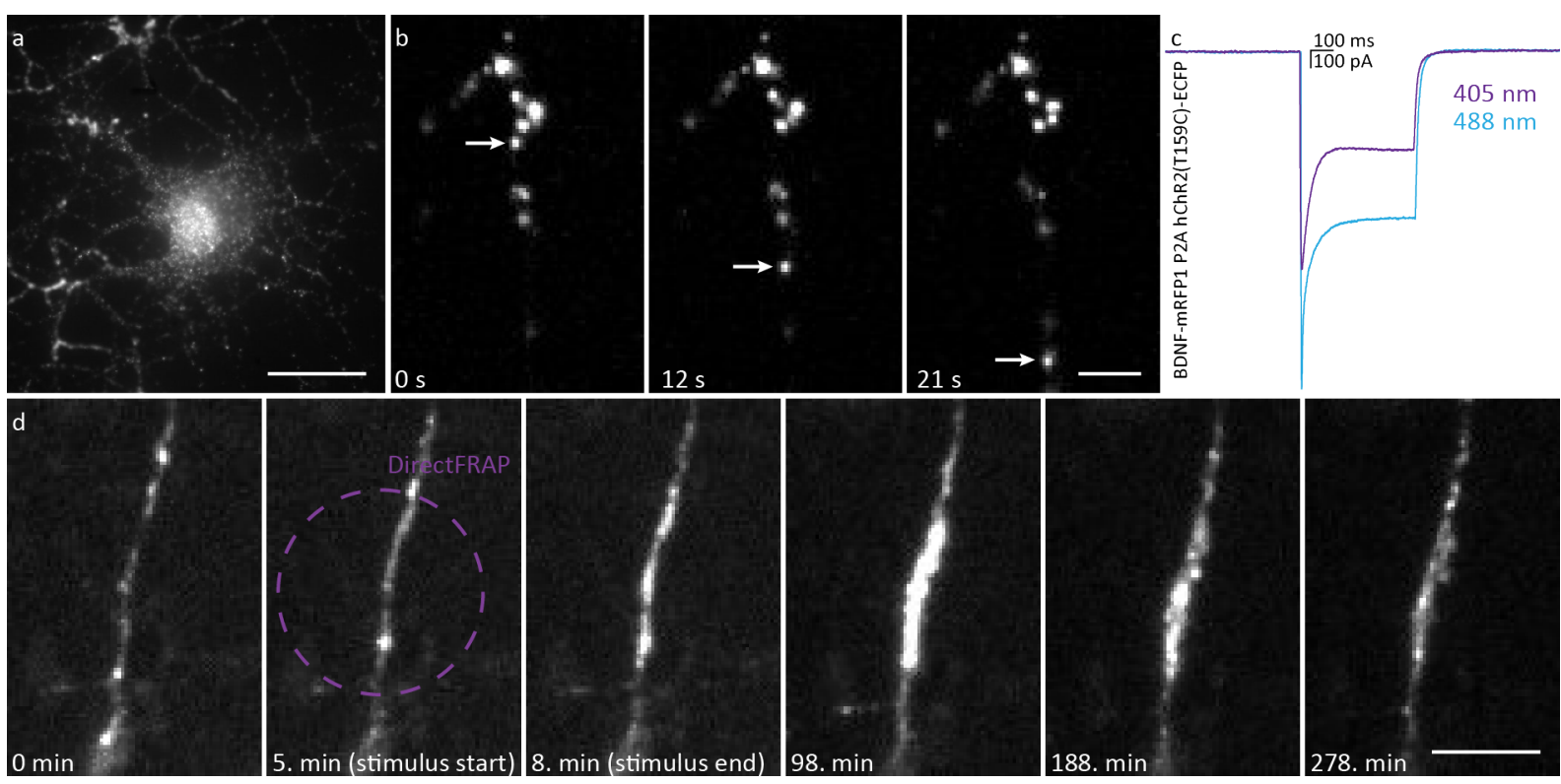

Figure 11: Fluorescent BDNF puncta trafficking and effects of focal optogenetic stimulation. Representative images of GFP tagged BDNF puncta in the cell soma (a) and trafficking through a neuronal process (b) acquired by epifluorescence microscopy. Modified representation of data published earlier (Dean et al., 2012). (c) 405 and $488 \mathrm{~nm}$ elicited photocurrents from a BDNF-mRFP1-P2A-ChR-ECFP expressing cell. (d) Focal $10.6 \mu \mathrm{m}$ $405 \mathrm{~nm}$ DirectFRAP stimulation of a BDNF-mRFP1-P2A-ChR(L132C)-ECFP expressing cell process and representative images before and after $3 \mathrm{~min}$ of $0.5 \mathrm{~Hz} 250 \mathrm{~ms} 405 \mathrm{~nm}$ light pulse stimulation. Scale bars are 50,3 and $5 \mu \mathrm{m}$ in panels $a, b$ and $d$, respectively. 


\section{BDNF-mRFP1 is released by neurons and transferred to nearby cells}

When we expressed BDNF-mRFP1 under the control of the beta actin promotor in dissociated hippocampal cultures, we observed a gradient of fluorescent signal, highest in very bright cells and weaker by distance to this cell, in other cell somata. We performed ICC experiments by labeling cultures for Map2 and GFAP, and found that some of the BDNF-mRFP1 signal was found in cells immunopositive for GFAP (Figure 12), which do not normally express proteins under control of the beta actin promoter at high levels.
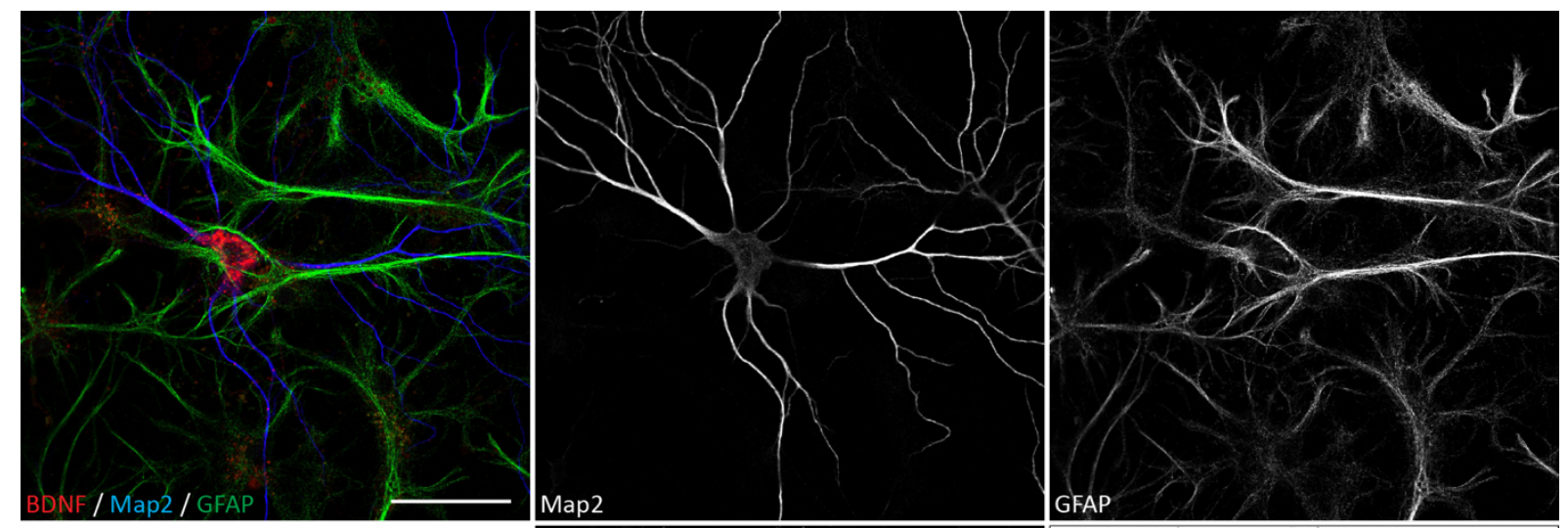

Figure 12: Map2 and GFAP immunocytochemical labeling of a PO derived dissociated hippocampal culture expressing BDNF-mRFP1. BDNF-mRFP1 produced by the neuron in the center can be found in nearby cells positive for GFAP. Black and white images show inverted signal of the individual channels. Scale bar represents $50 \mu \mathrm{m}$.
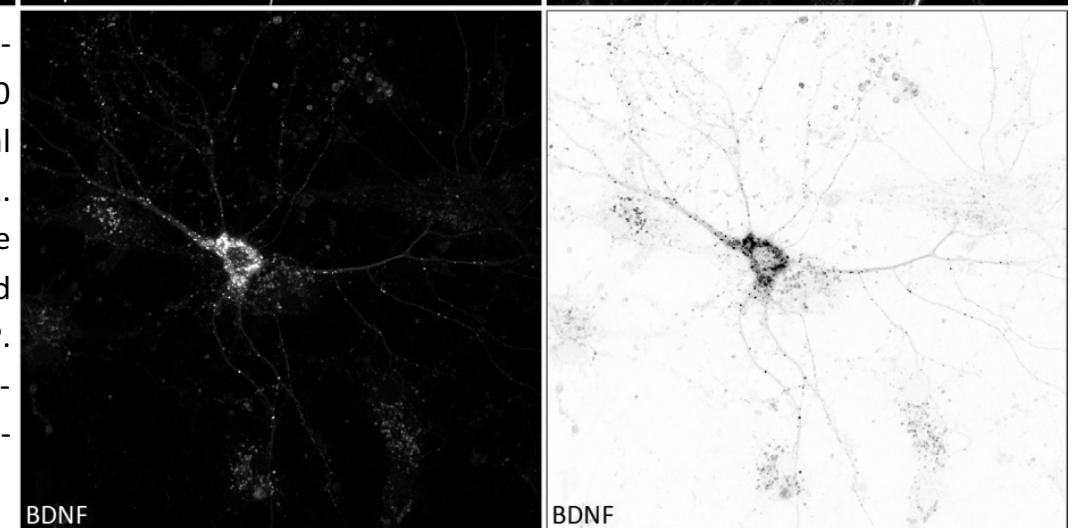

We also noticed that in immunostaining experiments, the BDNF-mRFP1 signal was much less than in live imaging conditions. To use live imaging and to exclude that BDNF-mRFP1 in astrocytes is actually produced in astrocytes, we transfected cells with a CaMKIla-BDNF-mRFP1-P2A-EGFP construct; expressing BDNF-mRFP1 under a promotor specific for glutamatergic neurons and labeling the transfected and producing cells with a cytosolic EGFP fluorophore. We then examined if BDNFmRFP1 transfer to neighboring cells occurs and if this could be detected in live and immunostained samples (Figure 13). Interestingly, we observed that there is indeed transfer of BDNF-mRFP1 to neighboring cells (negative for EGFP fluorescence). We also found, that BDNF-mRFP1 fluorescence signal in neighboring cells was significantly reduced by treatment with AraC (an antimitotic agent used to limit glial cell growth) from DIV7 onwards, fixing the culture with $4 \%$ PFA, permeabilizing (with the anionic detergent Triton X-100), and blocking (with donkey serum) for subsequent antibody staining. The combination of these effects led to an almost complete loss of BDNF-mRFP1 fluorescence. Washing cultures $3 x$ with PBS, as during immunocytochemistry, did not affect the fluorescence, providing evidence that BDNF-mRFP1 is not sticking to the surface of cells, where it can be simply washed away. When only EGFP was expressed, there was no detectable red fluorescence, 
thus the observed red fluorescent signal in neighboring cells is not auto-fluorescence or remaining fluorescent substances in the culture media like phenol red.
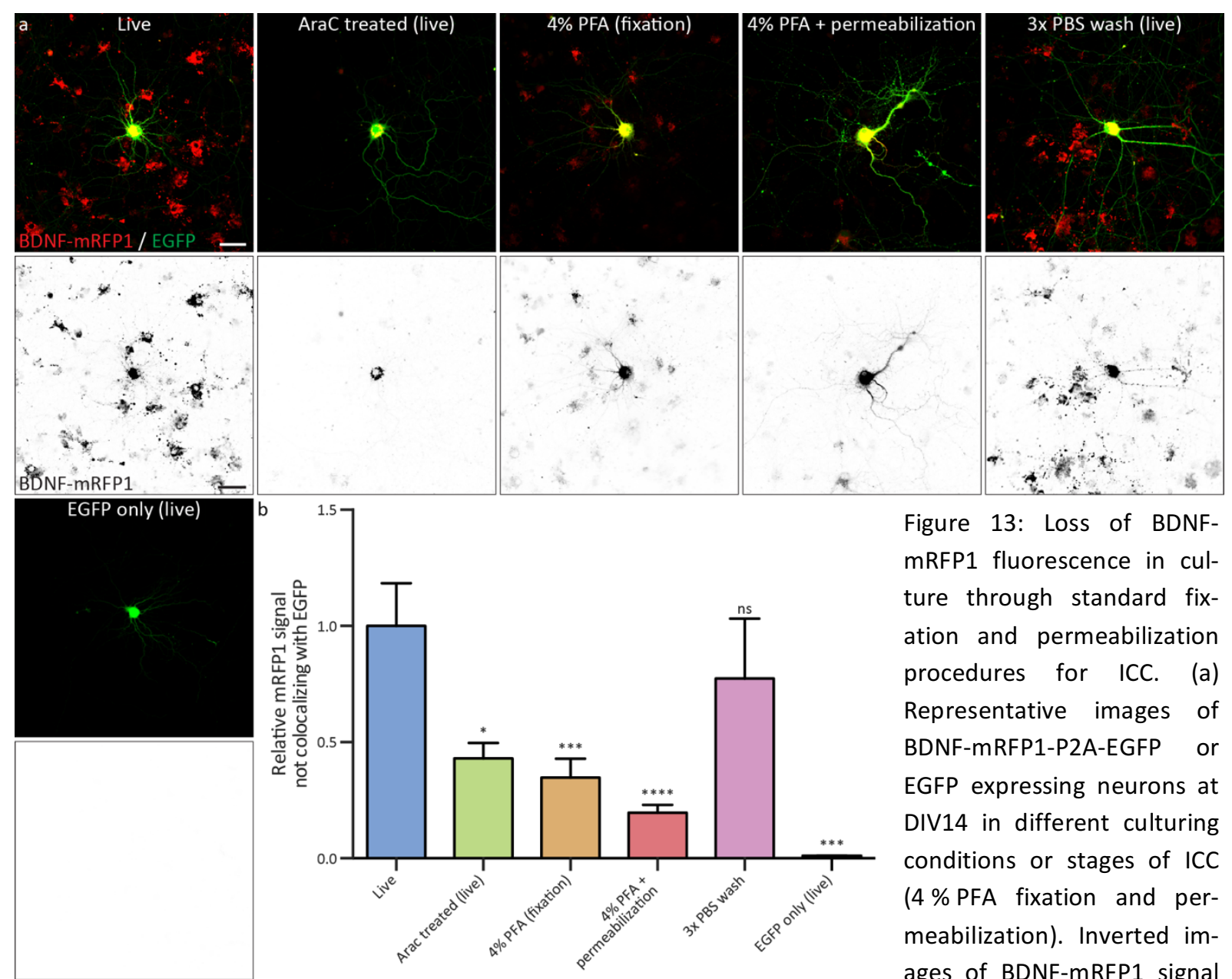

Figure 13: Loss of BDNFmRFP1 fluorescence in culture through standard fixation and permeabilization procedures for ICC. (a) Representative images of BDNF-mRFP1-P2A-EGFP or EGFP expressing neurons at DIV14 in different culturing conditions or stages of ICC (4\% PFA fixation and permeabilization). Inverted images of BDNF-mRFP1 signal

are shown below the respective fluorescence images. (b) Graphs represent averages and SEM of signal from 3 - 4 images of at least 2 - 5 individual cultures (In total Live $n=18$, AraC $n=13,4 \%$ PFA $n=18,4 \%$ PFA + permeabilization $n=18,3 x$ PBS wash $n=10$ and EGFP only $n=6)$. Statistical significance was determined by one-way ANOVA ( $p=0.0054)$ and individual differences were determined by Dunnett's multiple comparisons test $\left({ }^{*} P<0.05, * * P<0.01, * * * P<0.001\right.$ and $\left.* * * * P<0.0001\right)$. Scale bars represent $50 \mu \mathrm{m}$.

To exclude for specific fluorophore-mediated effects (i.e. mRFP1 versus EGFP), we compared the expression and distribution patterns in culture of BDNF-mRFP1-P2A-EGFP to BDNF-EGFP-P2A-mRFP1 and mRFP1-P2A-EGFP (Figure 14a - from left to right, two images per condition). All constructs expressed equally well and there was no significant mRFP1 fluorescence outside of the producing cell, when it was not fused to BDNF (Figure 14a, b). On the other hand, BDNF-EGFP signal spread was very weak and only a few cells could be detected at high detector gain levels. To test whether a potential acidic environment inside intracellular compartments might quench the EGFP fluorophore, we applied $25 \mathrm{mM} \mathrm{NH}_{4} \mathrm{Cl}$ to the culture to de-acidify internal compartments. This treatment significantly increased the EGFP fluorescence in neighboring cells (Figure 14c, d), indicating that BDNF-EGFP is also transferred to neighboring cells and is present predominantly in acidified compartments. 

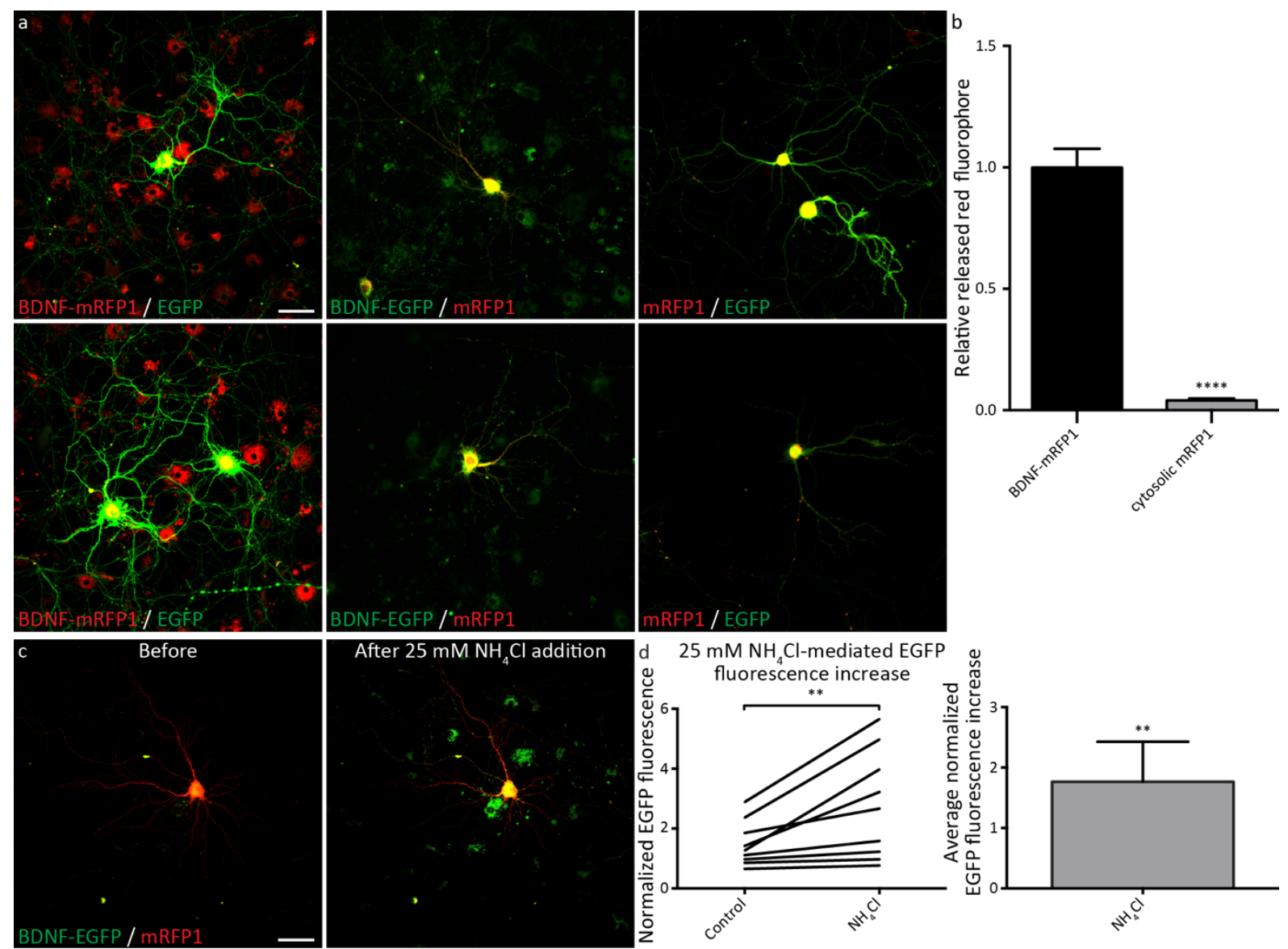

Figure 14: Assessment of fluorophore contribution to transfer. (a) Images show two examples each of hippocampal cultures at DIV20, expressing BDNF-mRFP1-P2A-EGFP (left), BDNF-EGFP-P2A-mRFP1 (middle) or mRFP1-P2A-EGFP (right). The corresponding graphs (b) represent averages and SEM of $n=6$ images per group (unpaired one-tailed t-test, $* * * * p<0.0001$ ). mRFP1 fluorophore is not transferred to neighboring cells, unless it is attached to BDNF. (c) BDNF-EGFP-P2A-mRFP1 expressing neurons at DIV16, before and after the addition of $25 \mathrm{mM} \mathrm{NH}_{4} \mathrm{Cl}$. The corresponding graphs (d) represent average EGFP intensities $(n=9)$ normalized to mean and their change after $25 \mathrm{mM} \mathrm{NH}_{4} \mathrm{Cl}$ addition (left) and the EGFP average intensity relative to control with SD (right) (paired one-tailed t-test, ${ }^{* *} \mathrm{p}=0.0052$ ). Both BDNF-mRFP1 and BDNF-EGFP transfer to neighboring cells occurs and is therefore not mediated by a specific fluorophore. Scale bars represent $50 \mu \mathrm{m}$.

The above experiments imply, that the observed transfer is not an artifact mediated by a specific fluorophore, but any tag on BDNF could affect its properties. To exclude this possibility, we transduced cultures with untagged BDNF delivered by AAV6 and expressed under synapsin promotor control for expression exclusively in neurons. BDNF was then detected using immunocytochemistry. BDNF virus application dramatically increased BDNF signal in cultures detected by antibody immunoreactivity (Figure 15a), confirming BDNF overexpression. To detect specific cell types, cultures were cotransduced with EGFP, also expressed under synapsin promotor control, to mark neurons (Figure 15b), or immunostained for GFAP to mark astrocytes (Figure 15c) or both (Figure 15d). BDNF immunoreactivity was not only confined to EFGP positive neurons, but was also detected in GFAP immunopositive cells, which are not reported to express BDNF themselves. Since there was no detectable EGFP in GFAP-positive cells, it can be inferred, that BDNF overexpression, was also exclusive to neurons and BDNF was transferred to astrocytes. 


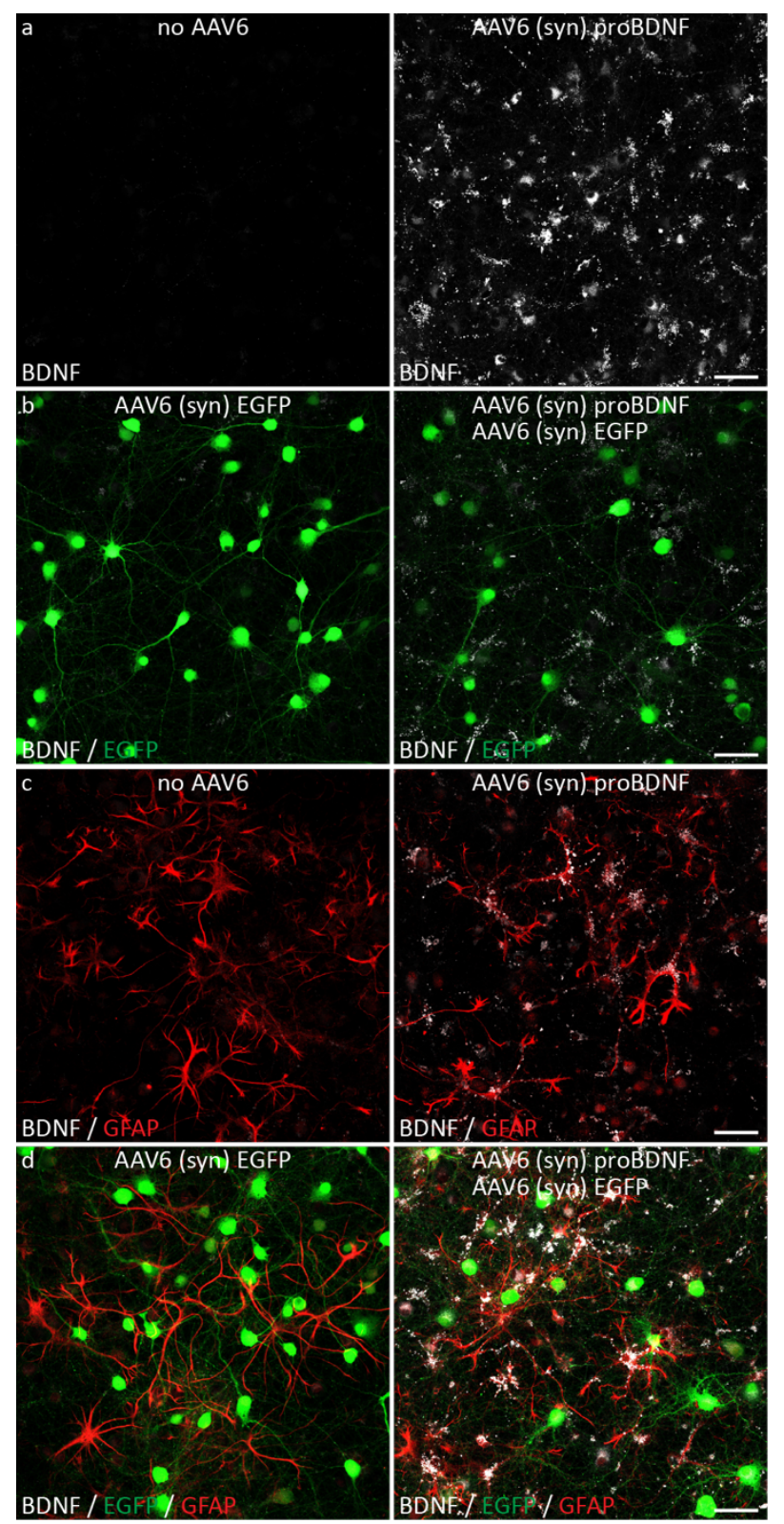

Figure 15: Fluorophore independent transfer of BDNF. (a) Images show BDNF immunoreactivity (white) in DIV14 dissociated hippocampal cultures, untreated (left) or transduced with AAV6, leading to expression of BDNF under synapsin promotor control (right). Additionally, cells were transduced with AAV6 EGFP under synapsin promotor control $(b, d)$ and/or were costained for $\operatorname{GFAP}(c, d)$. A strong increase in BDNF immunoreactivity was observed in the AAV6 BDNF overexpressing neurons, which is transferred to and colocalizes with GFAP immunopositive cells. The expression pattern and spread of signal appear similar for untagged BDNF and mRFP1-BDNF, indicating that transfer to neighboring cells is independent of an attached fluorophore. Scale bars represent $50 \mu \mathrm{m}$.

\section{Transferred BDNF-mRFP1 is taken up by target} cells

To test if the BDNF-mRFP1 that is transferred to neighboring cells is internalized by these cells, we immunostained for surface versus internal BDNF-mRFP1. As a control for a surface localized protein, we transfected cells with mRFP-Nrx, where the mRFP is extracellular. Immunostaining against RFP with a primary-secondary antibody preconjugate, coupled to AF647, without permeabilization confirmed the extracellular presence of mRFP in mRFP-Nrx transfected cells (Figure 16b). Secondary antibody application without primary antibody did not result in positive staining of the membrane (Figure 16a).

The same approach was used on BDNF-mRFP1-P2A-EGFP expressing cultures, where antibodies applied without permeabilizing cells, detected neither the cytosolic EGFP, nor overexpressed or transferred BDNF-mRFP1 (Figure 17b, c), confirming that the BDNF-mRFP1 signal transferred to neighboring cells is internal. The weak signal that can be detected is most certainly an amplification of a small amount of BDNF-mRFP1 that sits on the membrane or cover glass, or background signal from non-specific binding of the antibody. This signal however does not co-occur with the large BDNF-mRFP1 accumulations found in the cell somata. The observations that BDNF-mRFP1 is not easily washed away with PBS (Figure 13) and cannot be detected by RFP antibodies, when cells are not permeabilized, show that BDNF-mRFP1 is internalized upon release. 

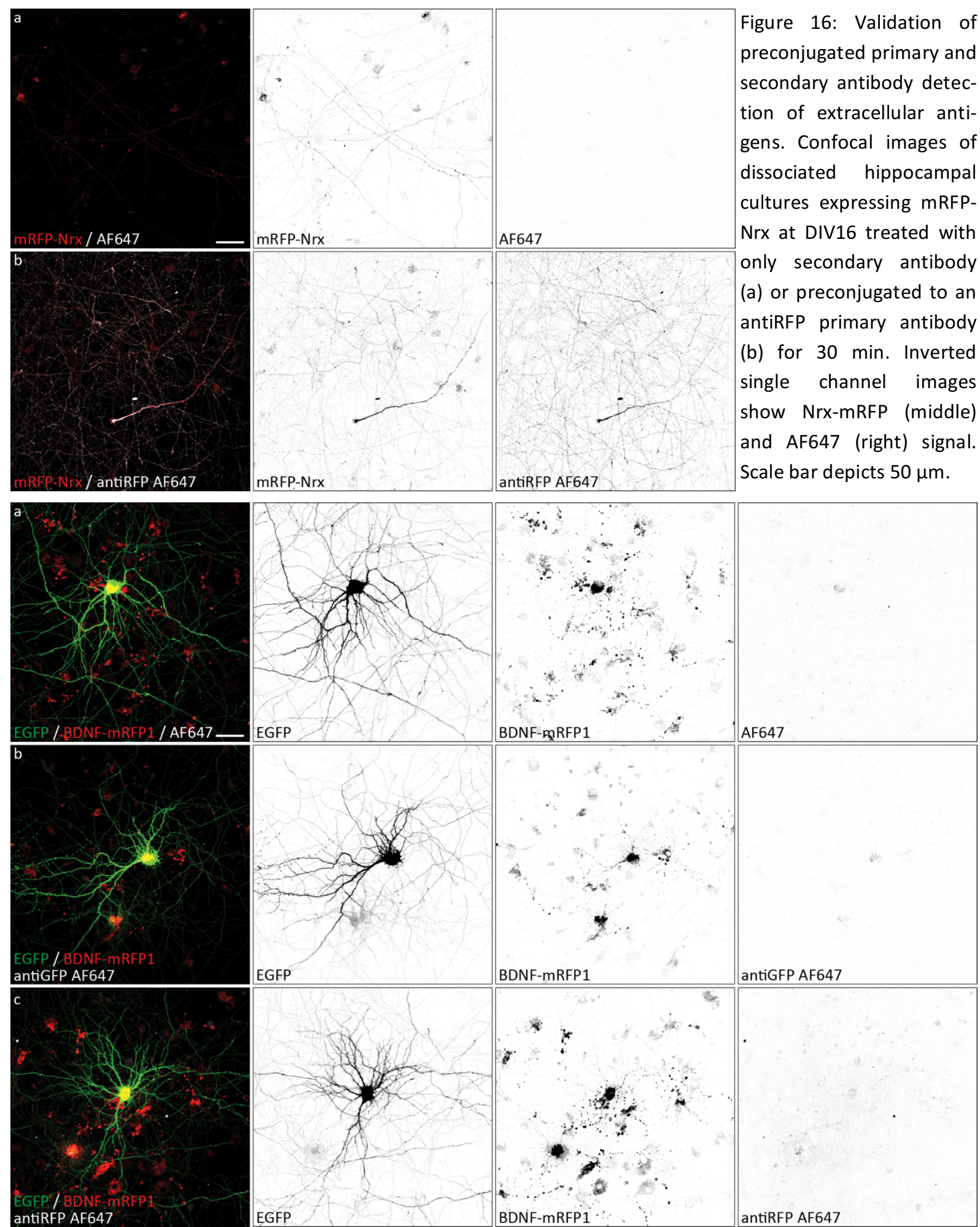

EGFP
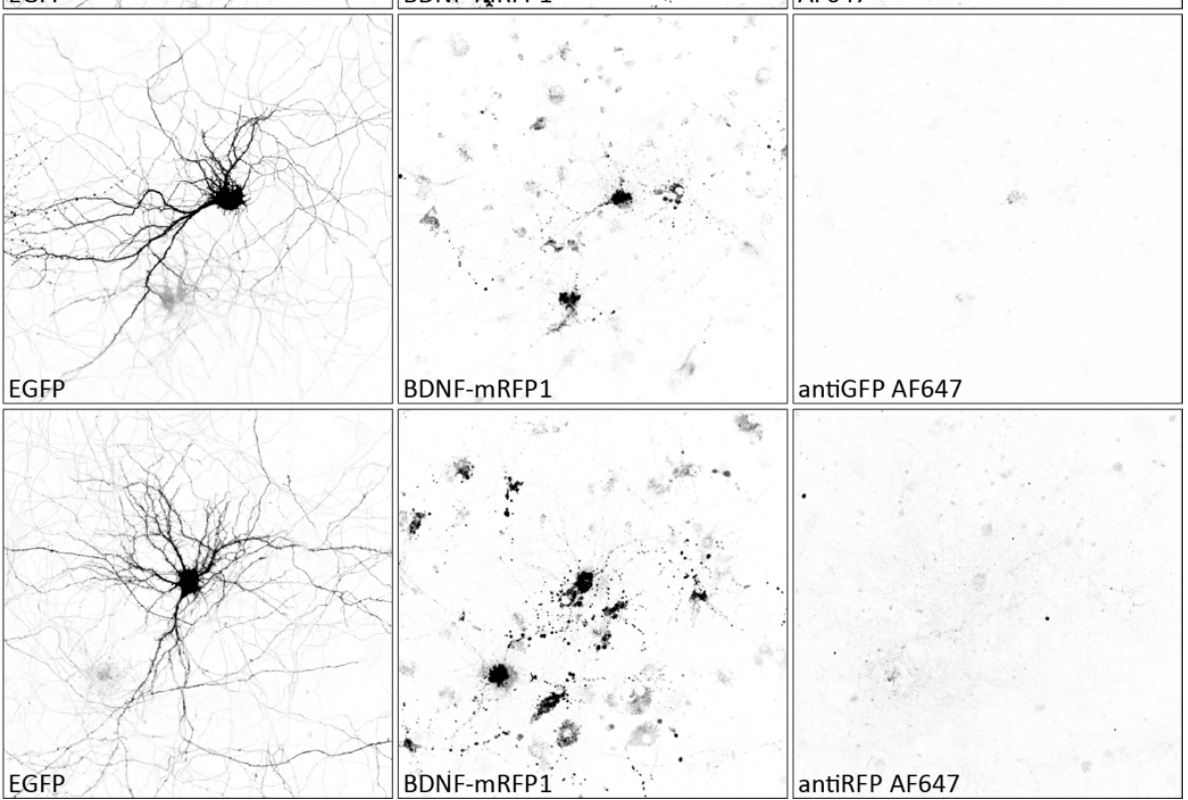

Figure 17: Transferred BDNF-mRFP1 is internalized. Confocal images of dissociated hippocampal cultures expressing BDNF-mRFP1-P2A-EGFP at DIV16 incubated with AF647 secondary antibody alone (a) or preconjugated to a primary antibody against GFP (b) or RFP (c). Inverted single channel images show ECFP, BDNF-mRFP1 and AF647 signal. Cytosolic localized EGFP and transferred BDNF-mRFP1 is inaccessible by primary-secondary antibody complexes added to the medium, indicating that BDNF-mRFP1 is internalized. Scale bar represents $50 \mu \mathrm{m}$. 


\section{Assessing transfer directionality of BDNF-mRFP1 in organotypic hippocampal slices}

To address the question of BDNF release from pre- or postsynaptic sites, we used organotypical hippocampal slices, providing an intact neuronal network, that is well studied, and where connectivity is known and preserved during preparation. We first injected AAV1/2 EGFP under synapsin promotor control in the hippocampal CA3 or CA1 region and found positive expression of EGFP that was mostly confined to neurons of the pyramidal cell layer in the area of injection (Figure 19a, b). We then injected AAV1/2 CaMKIla promotor controlled BDNF-mRP1-P2A-ECFP. To our surprise, no clear ECFP fluorescence could be found. But the sites of injection were clearly positive for BDNF-mRFP1, showing strong and locally confined fluorescence (Figure 19c, d). However, given the lack of ECFP fluorescence in the cells of origin, transfer of the BDNF-mRFP1 to another area in the hippocampus pre- or postsynaptic to the neurons in the injected area could not be confirmed. Nevertheless, it appeared that the spread of BDNF-mRFP1 fluorescence was not exclusively confined to the pyramidal cells found in the injected area, but could also be found in a large number of nearby cells, most likely predominantly astrocytes, that should not express the construct themselves via the CaMKIl $\alpha$ promotor (Figure 18). This implies that there is release of BDNF-mRFP1 and uptake by nearby cells in a dense and physiological environment.

Under the assumption, that the lack of ECFP fluorescence is caused by low expression and weak fluorescence, we exchanged the cytosolic fluorophore for EGFP and used purified AAV6 as carrier to increase local transduction efficiency. We also included a control for the transfer of BDNFmRFP1 by co-expression of cytosolic mRFP1 and EGFP. Expression and potential spread of BDNFmRFP1 could be observed (Figure 20a - d), but only a few EGFP positive cells could be found, and these were only faintly positive for BDNF-mRFP1 (Figure 20c, d). The injection of AAV6 carrying CaMKIl $\alpha$-mRFP1-P2A-EGFP let to locally confined expression with less spread of the mRFP1 fluorescence (Figure 20e), compared to BDNF-mRFP1. Thus, BDNF-mRFP1 likely spreads to neighboring astrocytes, but this remains to be confirmed. 


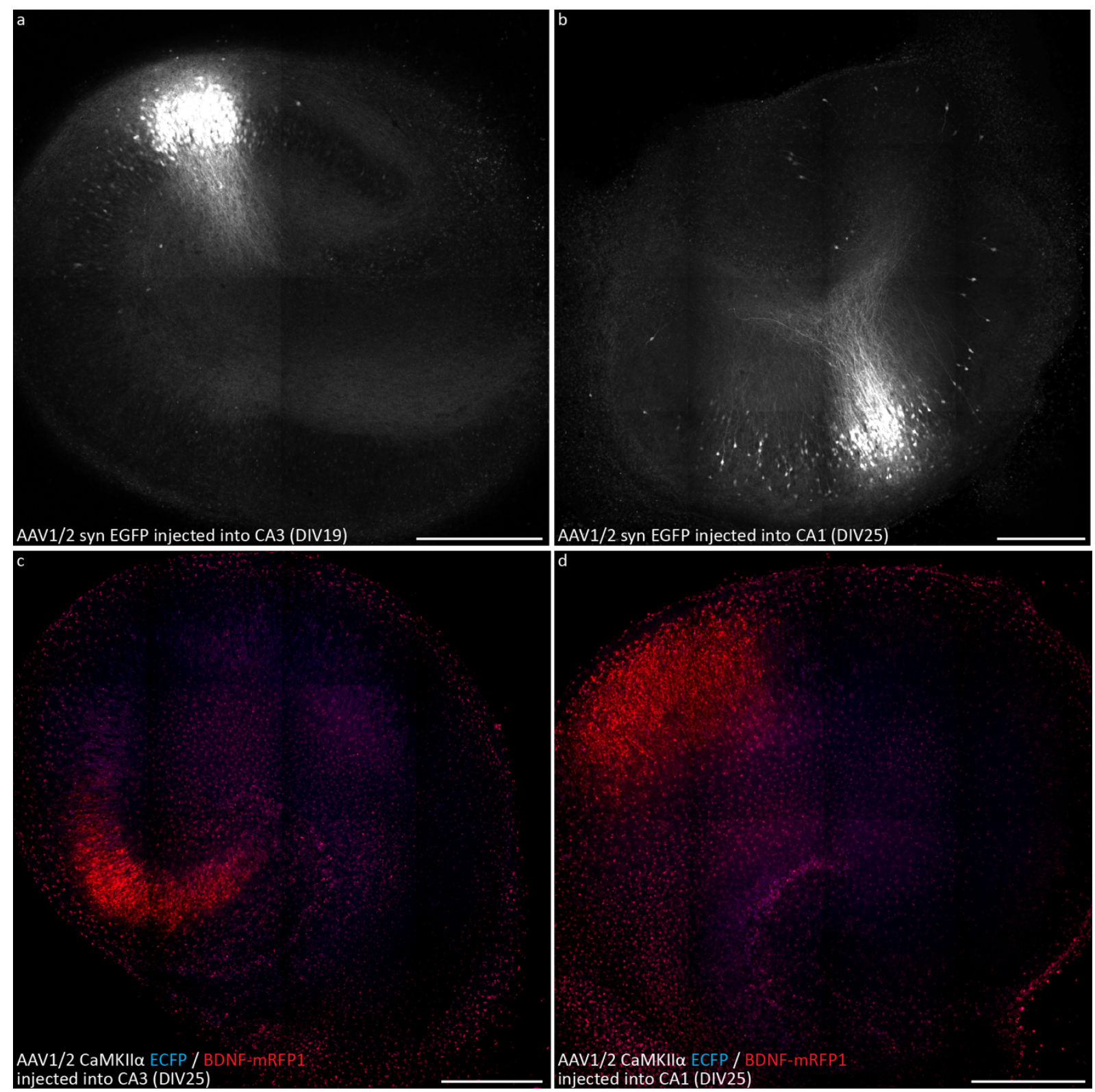

Figure 19: AAV-mediated overexpression of EGFP and BDNF-mRFP1-P2A-ECFP in the CA3 and CA1 regions of organotypic hippocampal brain slice (OHBS) cultures. Confocal images show slices expressing EGFP $(a, b)$ and BDNF-mRFP1-P2A-ECFP (c, d) in neuronal pyramidal cells of CA3 (a, c) or CA1 (b, d). Scale bars represent $400 \mu \mathrm{m}$.

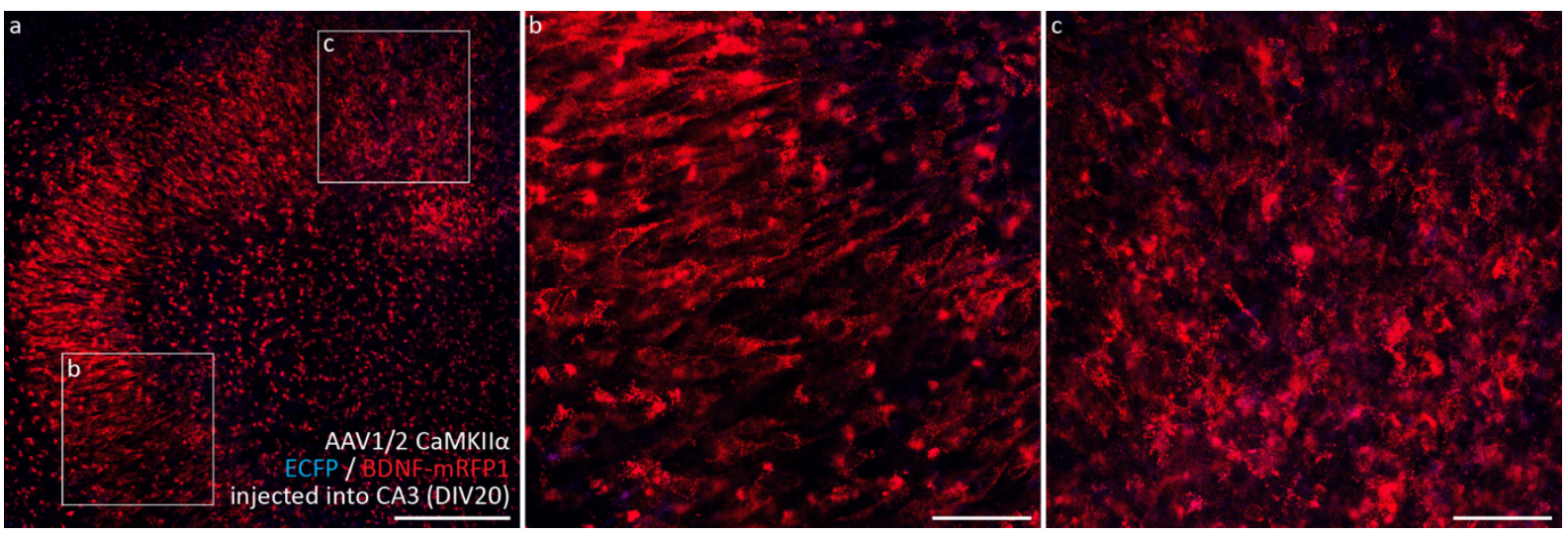

Figure 18: Spread of BDNF-mRFP1 signal and transfer to nearby cells in OHBS. Confocal image of transduced CA3 pyramidal cell layer expressing BDNF-mRFP1-P2A-ECFP (a). (b, c) Zoomed images of the two areas marked by squares. Scale bars represent 400 or $100 \mu \mathrm{m}$ respectively. 


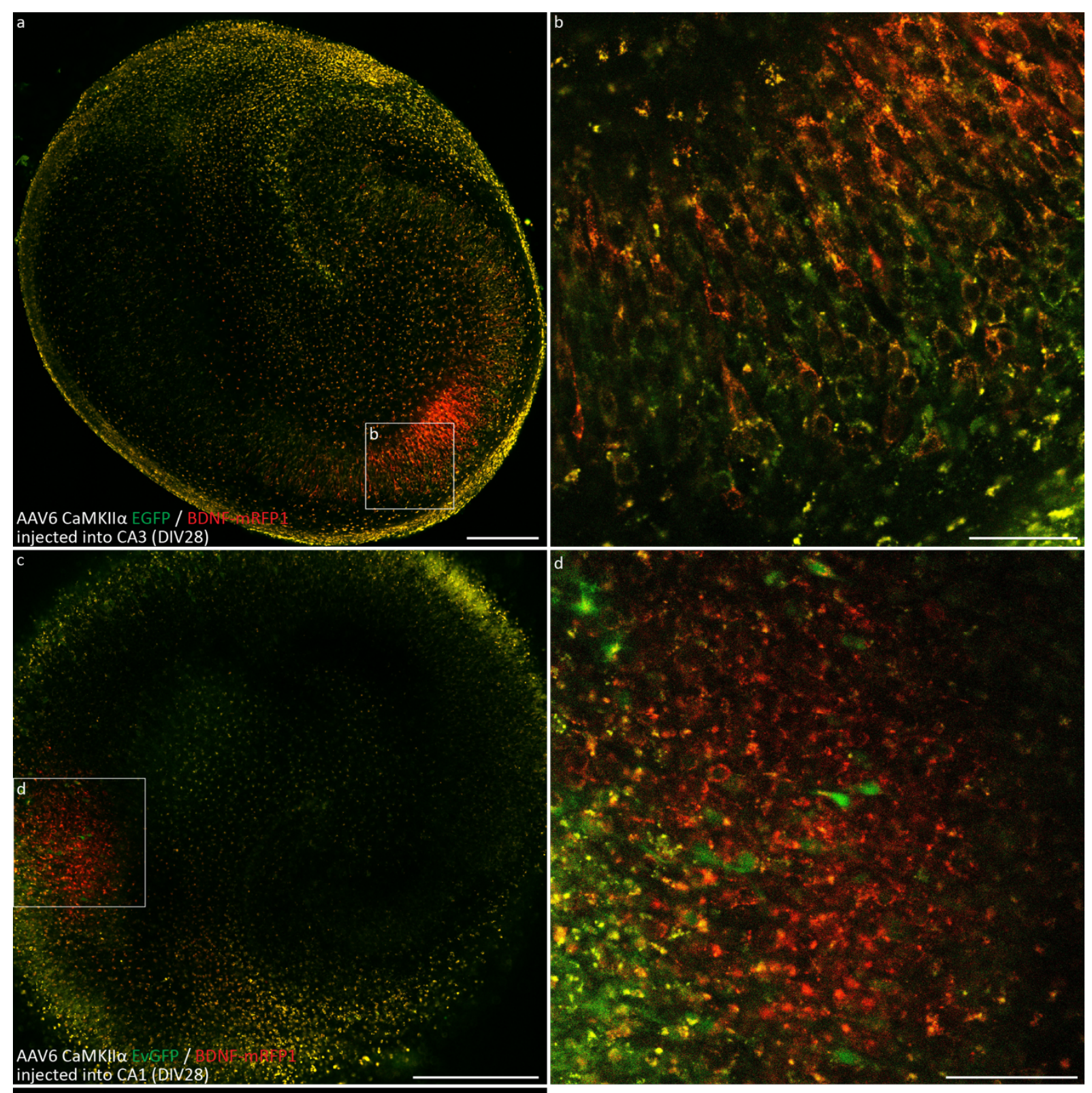

Figure 20: Injection of purified BDNF-mRFP1-P2A-EGFP or mRFP1-P2A-EGFP AAV6 to CA3 and CA1 in OHBS. Confocal images of slices injected in CA3 (a) and CA1 (c) regions. (b, d) Respective zoomed images of areas marked by squares. (e) OHBS expressing mRFP1-P2AEGFP. Scale bars represent $400 \mu \mathrm{m}$ for the images showing the whole hippocampus or $100 \mu \mathrm{m}$ for the respective zoomed image. 


\section{BDNF-mRFP1 uptake by different cell types}

Even though performing immunocytochemistry reduces RFP signal drastically, it can still be used to identify specific cell types containing large BDNF-mRFP1 accumulations. We performed immunocytochemistry experiments on BDNF-mRFP1-P2A-ECFP expressing cultures to identify specific cell types based on GFAP immunoreactivity (astrocytes), Map2 (neurons), NG2 (oligodendrocyte progenitor cells) and Iba1 (microglia). We found only little or no BDNF-mRFP1 in Map2 immuno-positive neurons (Figure 21a), some in NG2 immuno-positive oligodendrocyte progenitor cells (Figure 21b) and as reported earlier a substantial amount in GFAP immuno-positive astrocytes (Figure 21a - c). Unexpectedly, Iba1 stainings did not show any immuno-positive microglia (Figure 21c). Therefore, no assumptions can be made about uptake of BDNF-mRFP1 in microglia cells.

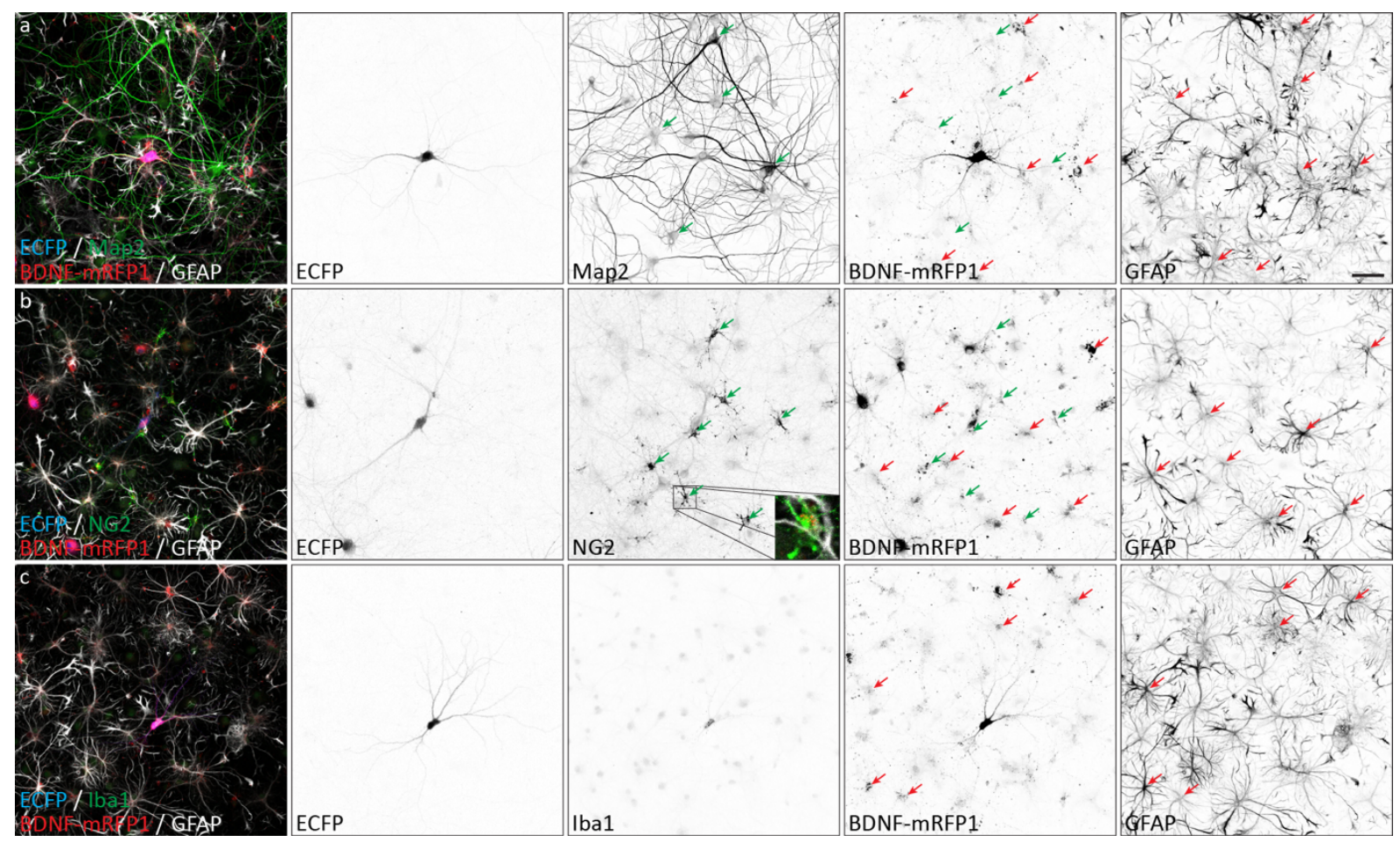

Figure 21: Assessment of BDNF-mRFP1 uptake by different cell types regularly found in dissociated hippocampal cultures at DIV14. Cultures expressing BDNF-mRFP1-P2A-ECFP were stained for Map2 and GFAP (a), NG2 and GFAP (b) or Iba1 and GFAP (c). Inverted single channel images show ECFP, Map2/NG2/Iba1, BDNF-mRFP1 and GFAP. Green or red arrows indicate soma of Map2-positive neurons or GFAP-positive astrocytes, respectively. Only traces of BDNF-mRFP1 signal were present in ECFP-negative Map2 immunopositive cells (a), however NG2 and GFAP immunopositive cells appear clearly positive for BDNF-mRFP1 signal (b). Iba1 immunostaining did not show any detectable cells. Scale bar represents $50 \mu \mathrm{m}$.

Because immunocytochemistry reduces detectable BDNF-mRFP1, we utilized AAVs to express different fluorophores under neuron (synapsin) and astrocyte (GFAP2.2) specific promotors for live imaging. We tested AAV transduction efficiencies and determined that our AAV1/2 syn-EGFP successfully transduces and labels $87.5 \pm 8.2 \%$ of Map2 positive cells (Figure 22a) and AAV1/2 GFAP2.2-EGFP labels $81.9 \pm 11.6 \%$ of GFAP immunopositive cells (Figure $22 \mathrm{~b}$ ). In addition we also co-immunostained for ALDH1L1, another astrocytic marker that labels both reactive and nonreactive astrocytes and found that only few cells were immunopositive for ALDH1L1, but not GFAP. 
We therefore combined BDNF-mRFP1-P2A-ECFP expression with AAV1/2 mediated GFAP2.2 promoter driven GFP and synapsin promoter driven LSSmOrange expression, to yield four color live labeling without the need for immunocytochemistry to identify cell types (Figure 22c). LSSmOrange (large stokes shift mOrange (Shcherbakova et al., 2012)) is excited by the same wavelength as EGFP, but emits in the orange/red spectrum and is thus separable from ECFP, EGFP and mRFP1 fluorescence. As shown by the individual channels (Figure 22d), LSSmOrange-expressing neurons, EGFP-expressing astrocytes, ECFP-labelled BDNF-mRFP1 producing cells and BDNF-mRFP1 signal could be easily distinguished. However, if EGFP fluorescence was very strong, it did also appear weakly in the LSSmOrange channel. But since the two fluorophores were expressed under the control of two exclusive promotors, the EGFP signal could in these cases be subtracted from the LSSmOrange channel. Using this approach, we determined that $10.1 \pm 7.3 \%$ of total released BDNF-mRFP1 was in neurons, $48.6 \pm 16.8 \%$ was in astrocytes and $36.5 \pm 10.8 \%$ did not co-occur with either GFP or LSSmOrange. These results varied between cultures, depending on cell density, transfection and transduction efficiency, usually with the consequence of more or less signal in the unassigned group, while the ratio between neurons and astrocytes remained constant.
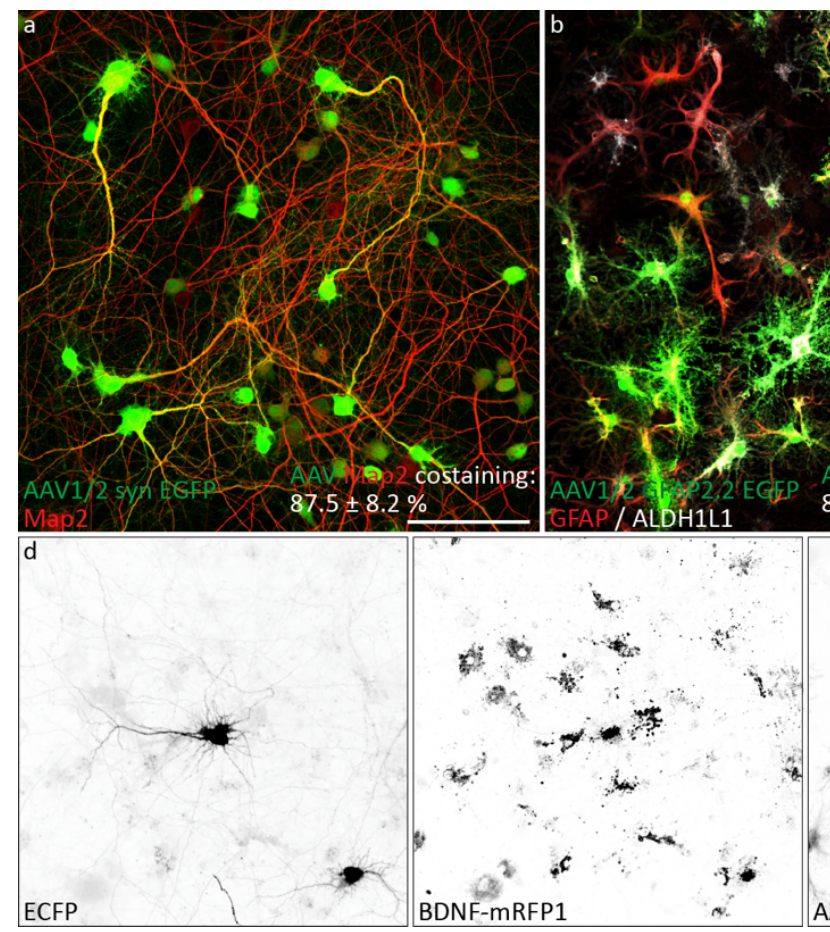
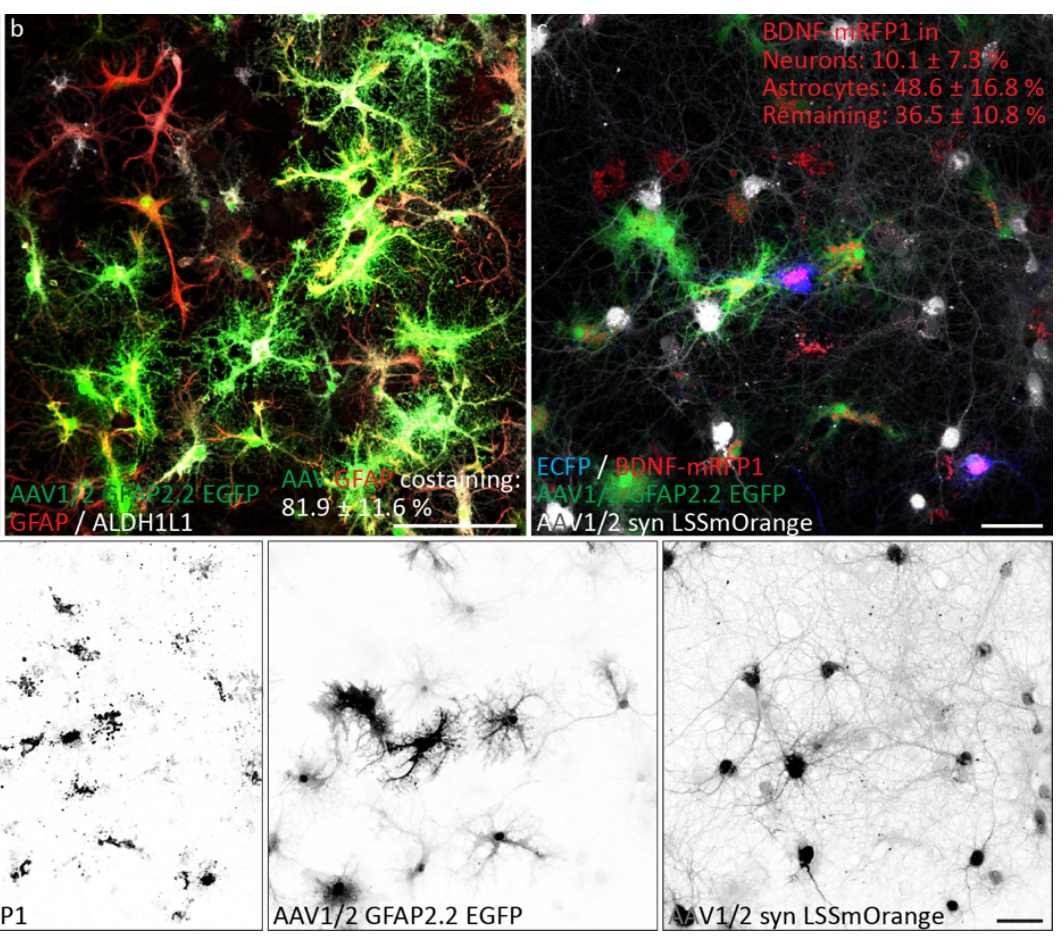

Figure 22: AAV-mediated live labeling of neurons and astrocytes. Cultures transduced with AAV1/2 virions for the expression of EGFP under synapsin (a) or GFAP2.2 (b) promotor control and tested for Map2 or ALDH1L1 and GFAP immunoreactivity on DIV14. EGFP was found in $87.5 \pm 8.2 \%$ of Map2 immunopositive (a) and $81.9 \pm 11.6 \%$ of GFAP immunopositive cells (b) ( $n=6$ for each condition). Few cells were positive for ALDH1L1 only and not GFAP. (C) AAV1/2 synapsin LSSmOrange and GFAP2.2 EGFP in a culture expressing BDNF-mRFP1-P2A-ECFP, allowing identification of individual cell type, where neurons are identified by LSSmOrange, astrocytes by EGFP and BDNF-mRFP1 producing cells by ECFP fluorescence, while the released BDNF-mRFP1 can be simultaneously observed (d). 10.1 $\pm 7.3 \%$ of the released BDNF-mRFP1 signal could be allocated to neurons, $48.6 \pm 16.8 \%$ to astrocytes and $36.5 \pm 10.8 \%$ to neither of these groups. Therefore, in dissociated hippocampal cultures, astrocytes but not neurons appear to be the primary recipient of BDNF-mRFP1 released by neurons. Scale bars represent $50 \mu \mathrm{m}$. 


\section{Subcellular localization of BDNF-mRFP1 taken up by astrocytes and neurons}

We were curious to find out where the released and taken up BDNF-mRFP1 goes within cells and whether it is degraded or even re-released. Therefore, we overexpressed fluorescently tagged EEA1, Rab5a, Rab7, Lamp1 and LC3 in neurons and astrocytes to mark early endosomes, late endosomes, lysosomes, and autophagosomes, respectively, using electroporation. As a first step, we verified the localization of these overexpression constructs by testing cells expressing the respective marker proteins for immunoreactivity of antibodies against EEA1, Rab5, Rab7 and Lamp1 (Figure 23); a suitable LC3 antibody was not available and LC3 could therefore not be tested. Marker proteins and antibodies stained the same intracellular puncta. Antibodies also labeled signal in nearby nontransfected cells and in some cases displayed slightly higher background signal than expression constructs, but in general the expression of subcellular localization marker proteins appears to be a reliable method to mark subcellular compartments.

Next, we electroporated cells with the different subcellular marker constructs, and subsequently transfected them with BDNF-mRFP1-P2A-ECFP and applied AAV1/2 virus mediating the expression of LSSmOrange under synapsin or GFAP2.2 promotor control. Cells having taken up BDNF-mRFP1 and transfected with one of the subcellular markers were identified by the presence of LSSmOrange and absence of ECFP fluorescence (Figure 24a). Colocalization was confirmed by determining individual Pearson product-moment correlation coefficients. Colocalization was relatively high for BDNF-mRFP1 and Rab7 (>0.5) and BDNF-mRFP1 and Lamp1 (>0.7) compared to BDNF-mRFP1 with Rab5 $(<0.2)$ or LC3 $(<0.2)$. This did not appear to differ between neurons and astrocytes (Figure 24b).

\section{Expression of TrkB in neurons increases neuronal and decreases astrocytic BDNF-mRFP1 uptake}

Even though the target receptor for mature BDNF, TrkB, is broadly present in hippocampal neurons (Yan et al., 1997), much of the BDNF-mRFP1 was found in astrocytes. Potentially BDNF is either released as in its precursor form, preferably binding to p75 receptor or astrocytes express more TrkB receptors than the neurons. To test this, we created AAV1/2 synapsin promotor controlled expression constructs of the TrkB receptor, tagged with either EGFP or LSSmOrange. To verify exclusive neuronal expression, we compared AAV1/2 mediated expression of TrkB-EGFP with EGFP in neurons (Figure 25a, b or d, e) or astrocytes (Figure 25c), with the signal of Map2 (Figure 25a, c, d) and GFAP (Figure 25b, e) in immunocytochemical stainings. Like synapsin promotor controlled EGFP expression, Trkb-EGFP expression was confined to Map2 immunopositive cells (Figure 25a, d) and did not overlap with GFAP immunoreactive cells (Figure 25b, e). Map2 immunostaining did not overlap with AAV1/2 mediated expression of EGFP under GFAP2.2 promotor control (Figure 25c).

We found that the accumulation of BDNF-mRFP1 fluorescence in neurons was dramatically increased in the cell soma of neurons expressing TrkB-EGFP in cultures transfected with BDNF-mRFP1-P2A-ECFP and transduced with AAV1/2 mediating expression of either EGFP (Figure 26a) or TrkB-EGFP (Figure 26b) under synapsin promotor control. 


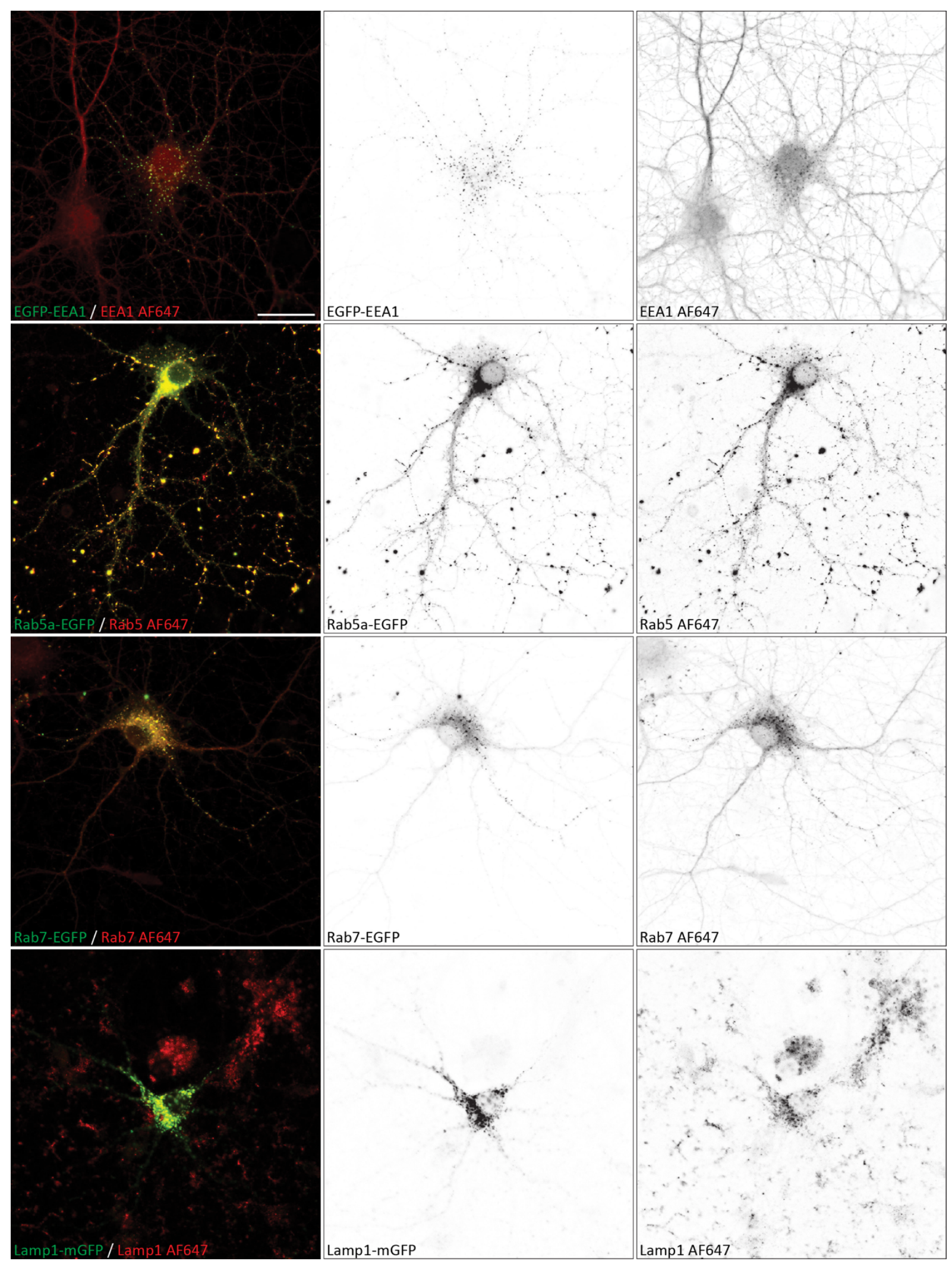

Figure 23: ICC validation of subcellular localization marker constructs for EEA1, Rab5a, Rab7 and Lamp1. Confocal images of dissociated hippocampal cultures at DIV13 expressing EGFP-EEA1, Rab5-EGFP, Rab7-EGFP or Lamp1-mGFP were tested for the immunoreactivity of the respective fluorescent puncta to the corresponding primary antibody. Primary antibodies were labeled with AF647-coupled secondary antibodies. In general, ICC stainings exhibited slightly elevated background signal, but detected the overexpressed subcellular marker proteins. Scale bar represents $25 \mu \mathrm{m}$. 

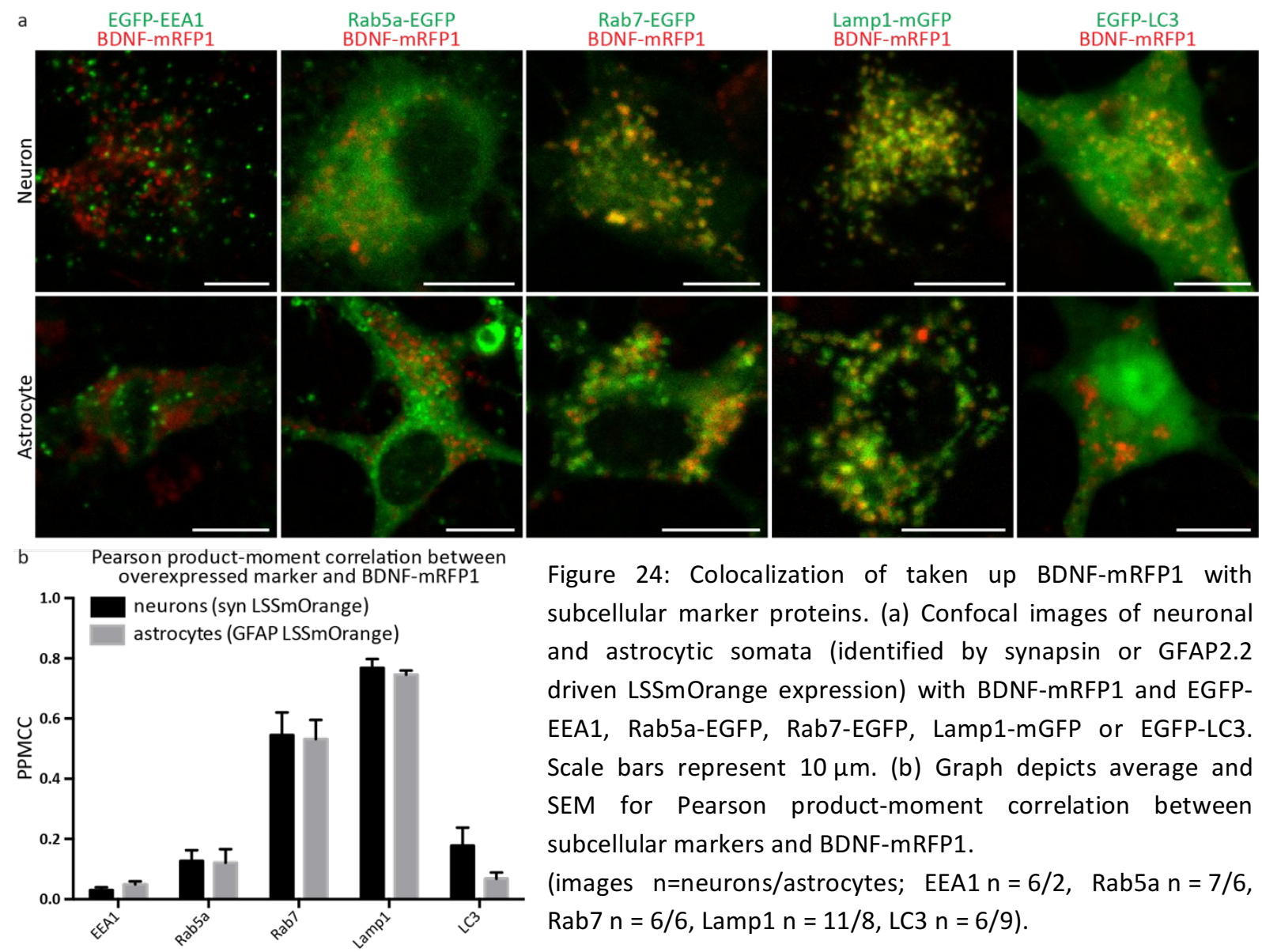

Figure 24: Colocalization of taken up BDNF-mRFP1 with subcellular marker proteins. (a) Confocal images of neuronal and astrocytic somata (identified by synapsin or GFAP2.2 driven LSSmOrange expression) with BDNF-mRFP1 and EGFPEEA1, Rab5a-EGFP, Rab7-EGFP, Lamp1-mGFP or EGFP-LC3. Scale bars represent $10 \mu \mathrm{m}$. (b) Graph depicts average and SEM for Pearson product-moment correlation between subcellular markers and BDNF-mRFP1.

(images $n=$ neurons/astrocytes; EEA1 $n=6 / 2$, Rab5a $n=7 / 6$, Rab7 $n=6 / 6$, Lamp1 $n=11 / 8$, LC3 $n=6 / 9$ ).
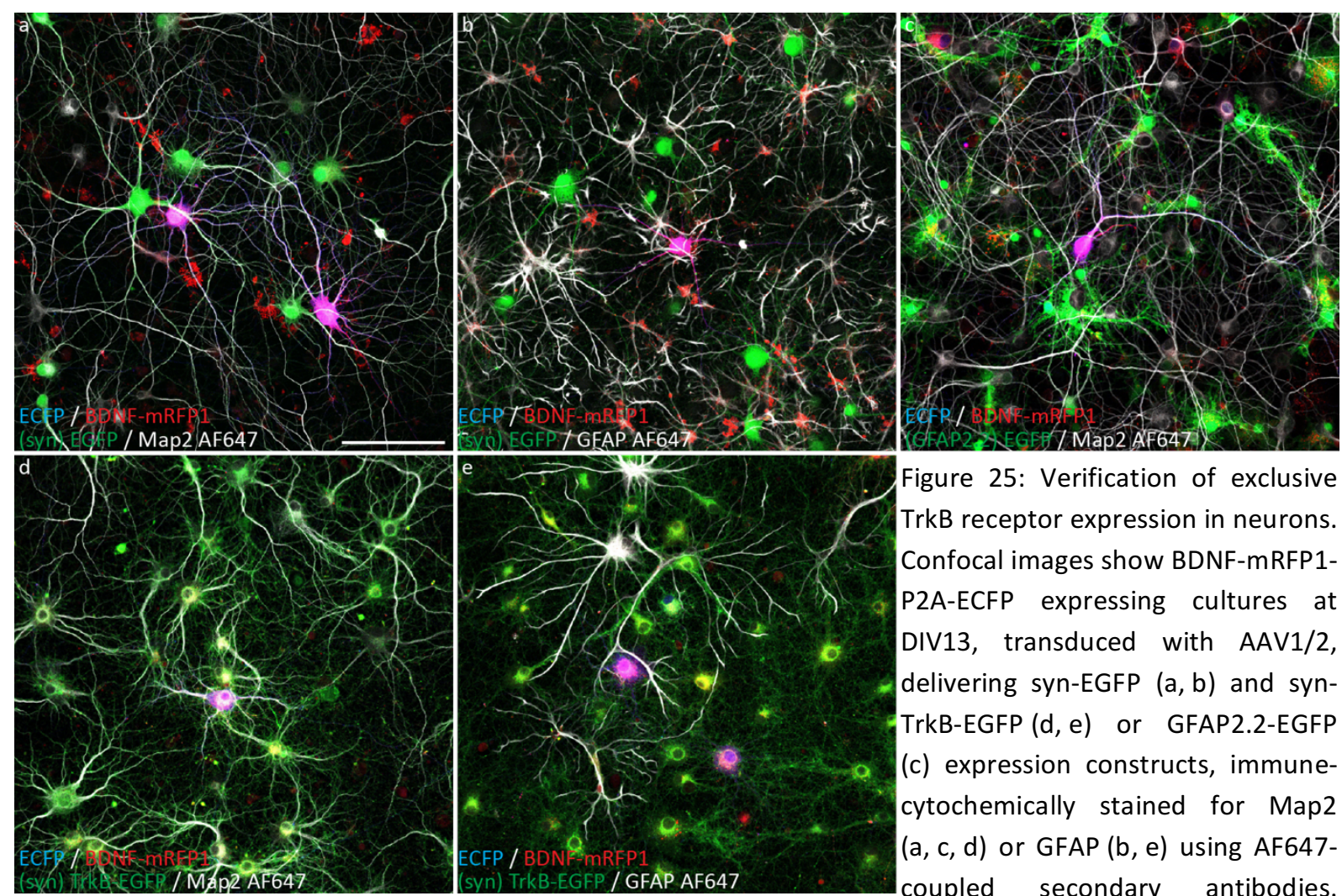

Figure 25: Verification of exclusive TrkB receptor expression in neurons. Confocal images show BDNF-mRFP1P2A-ECFP expressing cultures at DIV13, transduced with AAV1/2, delivering syn-EGFP $(a, b)$ and synTrkB-EGFP (d, e) or GFAP2.2-EGFP (c) expression constructs, immunecytochemically stained for Map2 (a, c, d) or GFAP (b, e) using AF647coupled secondary antibodies.

Synapsin promotor controlled EGFP and TrkB-EGFP were only detected in Map2 (a, d), but not GFAP (b, e) immunoreactive cells and GFAP2.2 promotor active cells were not immunoreactive for Map2 (c). Scale bar represents $100 \mu \mathrm{m}$. 


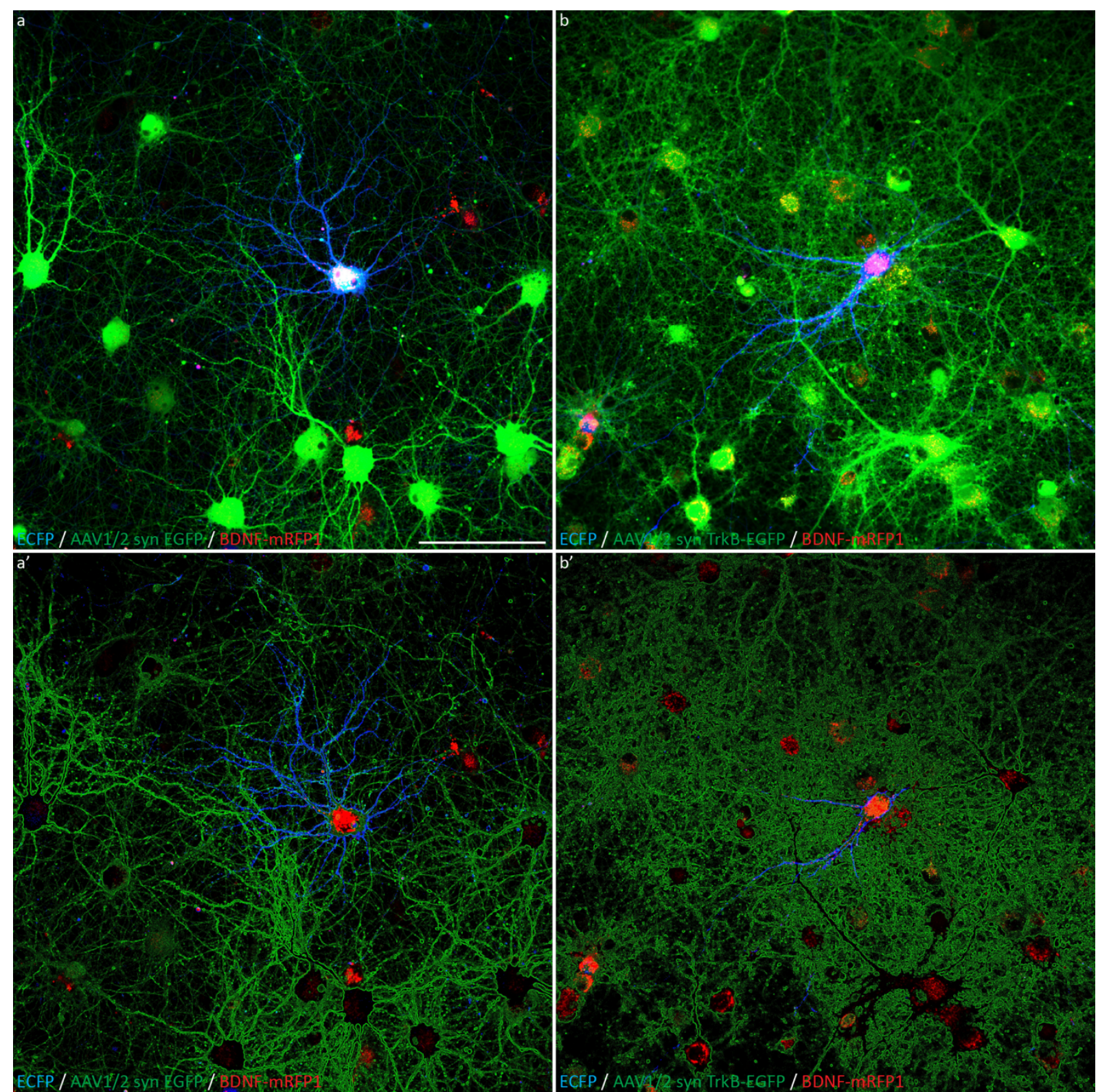

Figure 26: TrkB overexpression in neurons increases BDNF-mRFP1 uptake. Confocal images show DIV16 dissociated hippocampal cultures transfected with BDNF-mRFP1-P2A-ECFP and transduced with AAV1/2 delivering either syn-EGFP (a) or syn-TrkB-EGFP (b). For clarity EGFP fluorescence signal was partly removed from cell bodies $\left(a^{\prime}, b^{\prime}\right)$ by applying a standard Sobel edge detection filter. Individual TrkB-EGFP positive cell somata contain markedly enhanced levels of BDNF-mRFP1 compared to only EGFP expressing cells. Scale bar represents $100 \mu \mathrm{m}$.

We then tested, if enhanced neuronal BDNF-mRFP1 uptake by expression of TrkB in neurons has an effect on astrocytic BDNF-mRFP1 uptake. For this, we combined BDNF-mRFP-P2A-ECFP expression with AAV1/2 mediated expression of EGFP in astrocytes and LSSmOrange with (Figure 27b) or without TrkB (Figure 27a) in neurons. As observed before, neuronal cell soma contained markedly more BDNF-mRFP1, when they expressed TrkB-LSSmOrange, but conversely, astrocytes now contained substantially less BDNF-mRFP1 in their cell somata (Figure 28). 


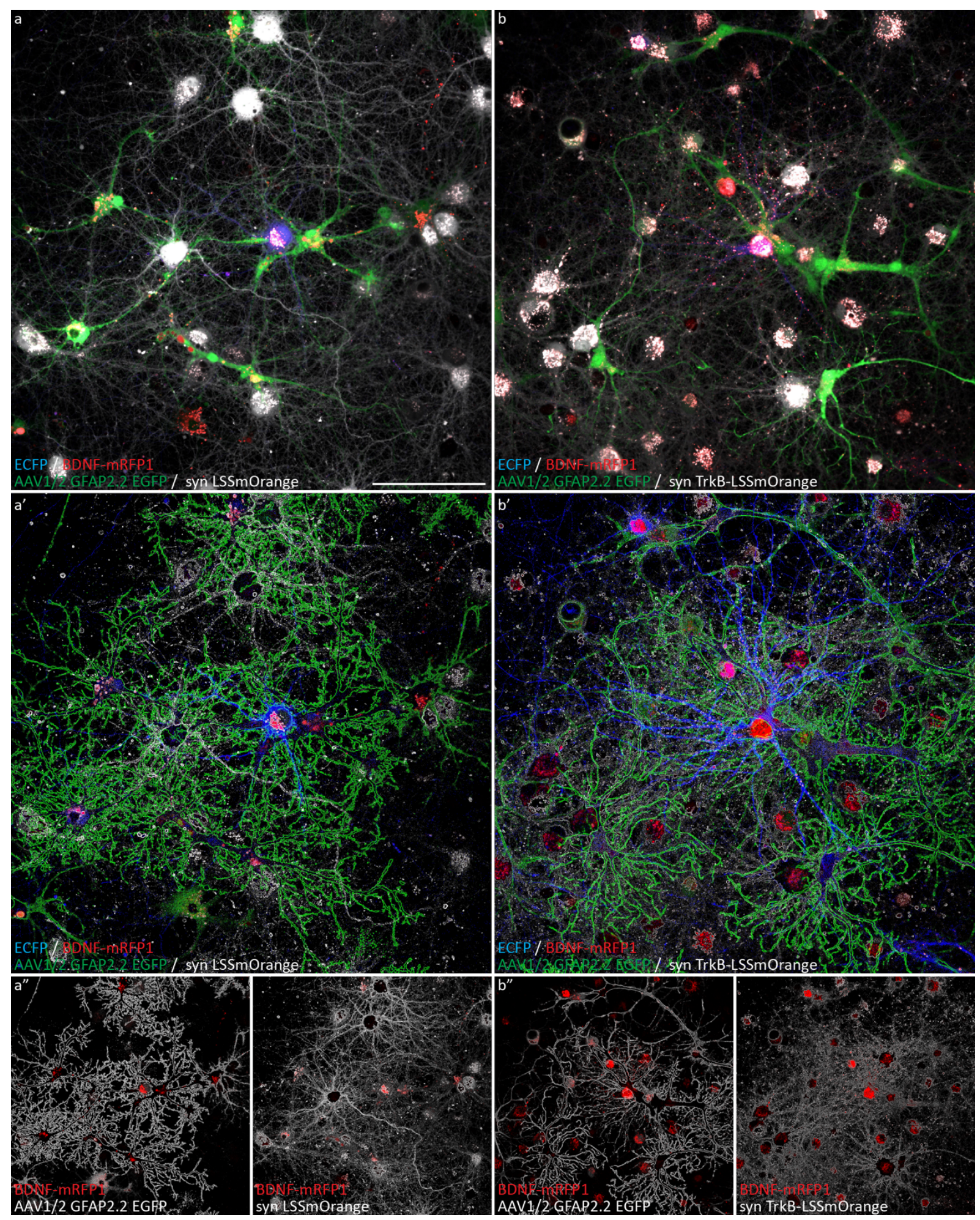

Figure 27: TrkB overexpression in neurons increases BDNF-mRFP1 uptake by neighboring neurons and reduces BDNF-mRFP1 found in astrocytes. Confocal images of dissociated hippocampal cultures at DIV16, expressing BDNF-mRFP1-P2A-ECFP and transduced with AAV1/2 GFAP2.2-EGFP and either syn-LSSmOrange (a) or syn-TrkB-LSSmOrange (b). For clarity EGFP and LSSmOrange fluorescence was partly removed from cell bodies $\left(a^{\prime}, b^{\prime}\right)$ by applying a standard Sobel edge detector filter. (a") and ( $\left.b^{\prime \prime}\right)$ show separated GFAP2.2 - EGFP and synapsin - LSSmOrange positive cells with BDNF-mRFP1. BDNF-mRFP1 signal increased in neurons and decreased in astrocyte somata due to TrkB overexpression in neurons. Scale bar represents $100 \mu \mathrm{m}$. 


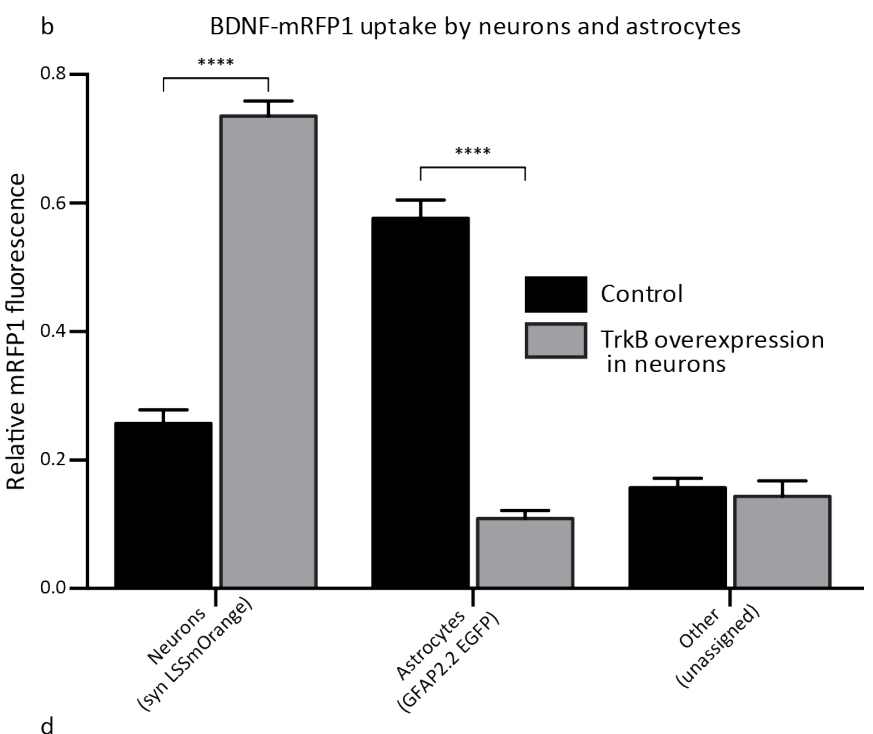

C
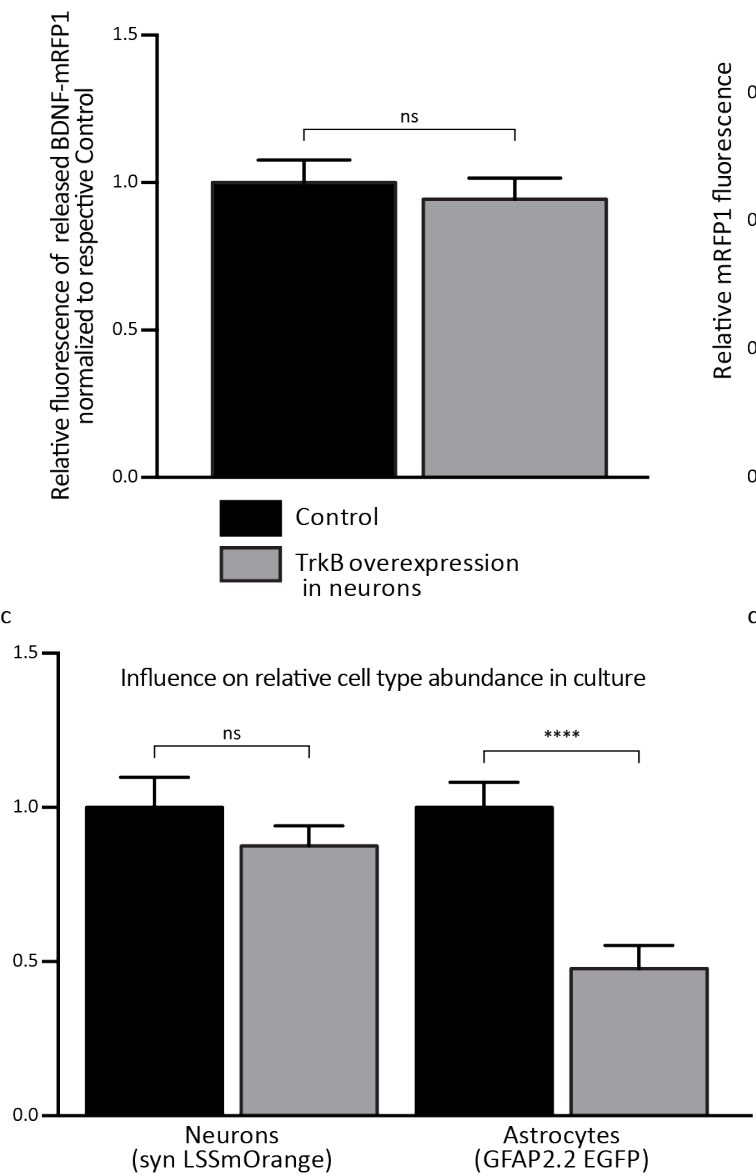

Average BDNF-mRFP1 found in the individual cell type, normalized to cell type abundance, total released BDNF-mRFP1 and control
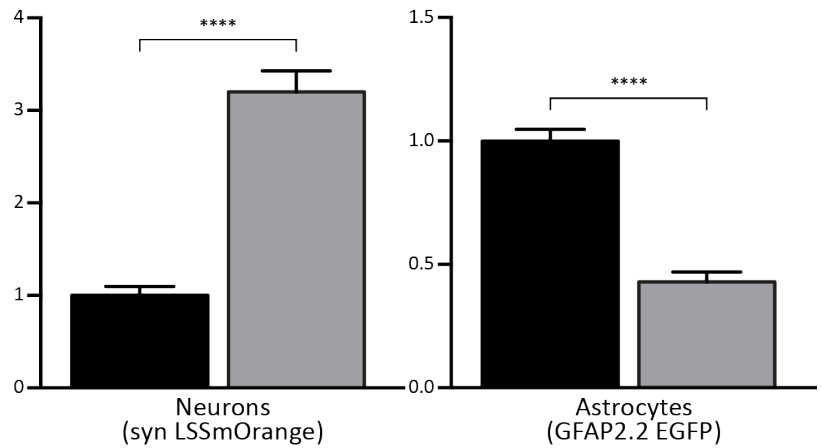

Figure 28: Changes in BDNF-mRFP1 uptake due to TrkB overexpression. Graphs (a - d) represent averages and SEM for values extracted from confocal images (Control $n=23$, TrkB overexpressing $n=22$; two separate cultures). There was no difference in the amount of released BDNF-mRFP1, between Control and the TrkB overexpression within individual cultures. (b) TrkB overexpression in neurons, increased the relative amount of BDNF-mRFP1 in neurons from $25.69 \%$ to $73.55 \%$ and decreased BDNF-mRFP1 in astrocytes from $57.65 \%$ to $10.87 \%$. BDNF-mRFP1 uptake remained roughly the same for the unassigned group of cells $(15.72 \%$ to $14.34 \%)$. The abundance of astrocytes, measured by surface coverage of the respective fluorophore, dropped significantly by $52.15 \%$ in cultures, in which TrkB was over-expressed (c). However, the effect of differential increased BDNF-mRFP1 uptake in neurons and decreased uptake in astrocytes persisted when average BDNF-mRFP1 in cells was normalized to cell abundance and total released BDNF-mRFP1 (d). Differences between indicated groups were tested for statistical significance, using unpaired two-tailed t-test, **** $\mathrm{P}<0.0001$, (a) $\mathrm{P}=0.6018$, (b) Other $\mathrm{P}=0.6183$, (c) Neurons $\mathrm{P}=0.2997$. 
It is important to note that there was no difference in the amount of released BDNF-mRFP1 between control and TrkB-LSSmOrange expressing cultures (Figure 28a). We found, that the relative uptake of total released BDNF-mRFP1 by neurons increased from $25.69 \%$ in control cultures to $73.55 \%$ in TrkB-overexpressing cultures and BDNF-mRFP1 uptake decreased from $57.65 \%$ in control cultures to $10.87 \%$, when TrkB-LSSmOrange was expressed in neurons (Figure 28b). Interestingly, we also noticed that the abundance of astrocytes decreased by $52.15 \%$ in cultures, in which TrkB was overexpressed in neurons, as indicated by the coverage of GFAP - EGFP fluorescence (Figure 28c). This could in part affect the observed changes in relative uptake. We therefore normalized the average BDNF-RFP1 fluorescence to the individual cell type abundance and total released BDNF-mRFP1 of the respective control experiment. Though less pronounced, the change in relative uptake by neurons and astrocytes was still preserved. BDNF-mRFP1 fluorescence in individual neurons increased threefold and decreased by $57.05 \%$ in astrocytes (Figure 28d), in TrkB overexpressing cultures compared to control.

\section{Influence of BDNF-mRFP1 on astrocyte abundance in dissociated hippocampal cultures}

We then further investigated the above reported effect of BDNF-mRFP1 on astrocyte abundance in culture. To further increase BDNF-mRFP1 release, we tested if $10 \mathrm{mM} \mathrm{KCl}$ or cLTP (by adding $10 \mu \mathrm{M}$ bicuculine, $200 \mu \mathrm{M}$ glycine, $50 \mu \mathrm{M}$ forskolin, $100 \mathrm{nM}$ rolipram) stimulation for 8 - 10 days had an influence on released BDNF-mRFP1 levels. We found a small increase in released BDNF-mRFP1 for $10 \mathrm{mM} \mathrm{KCl}$ stimulation, but a strong enhancement in released BDNF-mRFP1 for cLTP stimulation (Figure 29a). When cultures were stimulated using CLTP, BDNF-mRFP1 allocated to neurons and the unassigned population was reduced, but BDNF-mRFP1 increased in astrocytes (Figure 29b). While the abundance of neurons identified by LSSmOrange fluorescence remained stable, there was a tendency for $10 \mathrm{mM} \mathrm{KCl}$ stimulation and a clear effect for CLTP stimulation to increase the abundance of astrocytes in the culture identified by EGFP signal (Figure 29c). We found that the relative amount of BDNF-mRFP1 per cell did not change for neurons in the different conditions, but significantly decreased for the astrocytic population, when average BDNF-mRFP1 signal found in neurons or astrocytes was normalized to the respective cell type abundance and total released BDNF-mRFP1 of the corresponding control experiment (Figure 29d). 

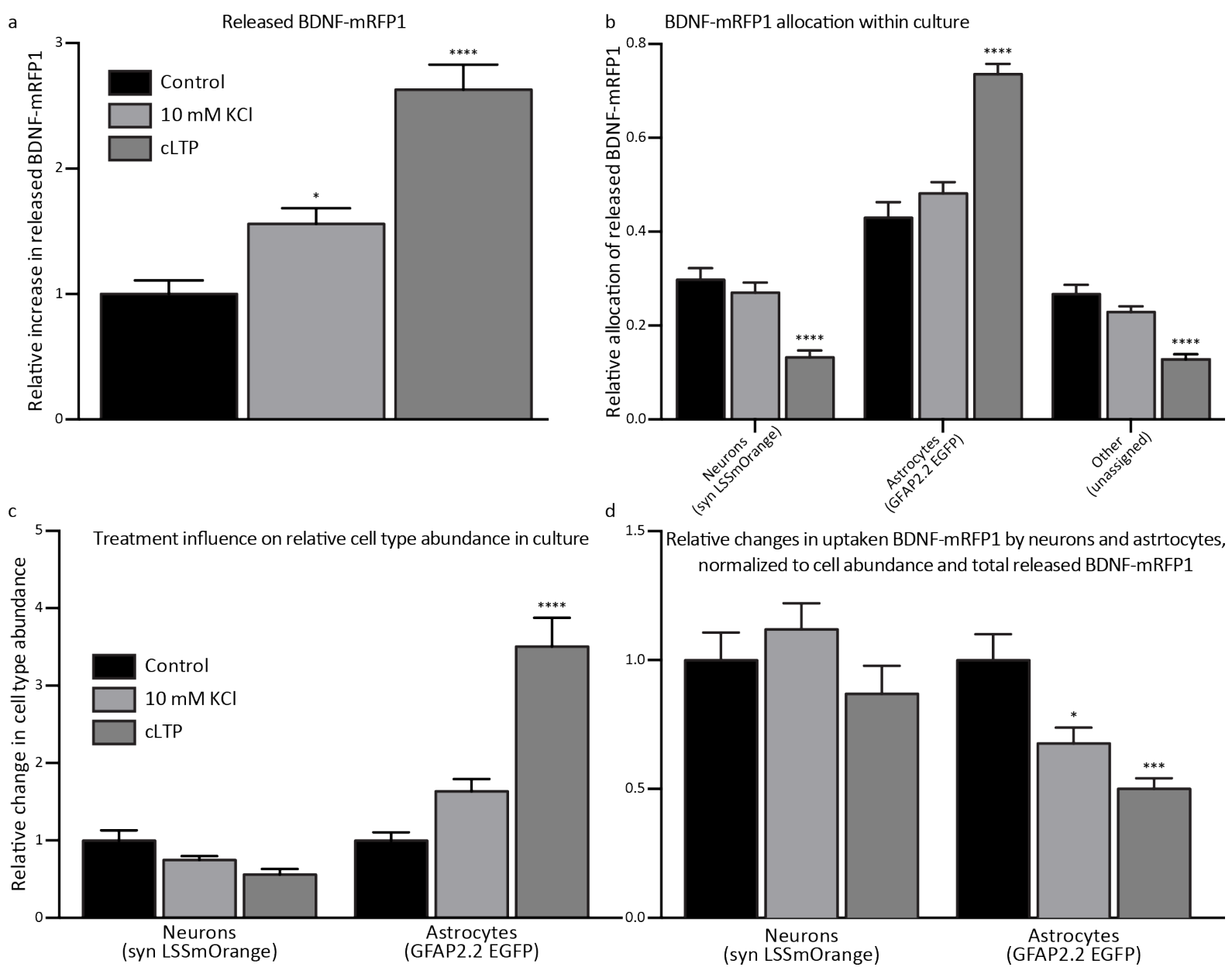
c
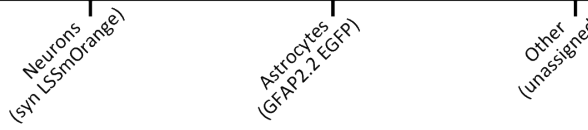

${ }^{1.5} 7 \begin{gathered}\text { Relative changes in uptaken BDNF-mRFP1 by neurons and astrtocytes, } \\ \text { normalized to cell abundance and total released BDNF-mRFP1 }\end{gathered}$

Figure 29: Influence of stimulated BDNF release on astrocyte abundance. Graphs represent averages and SEM of fluorescence intensities and distributions extracted from 5 - 10 confocal images per condition and of three separate cultures (Control $n=25,10 \mathrm{mM} \mathrm{KCl} n=30, \quad c L T P n=30$ ), expressing BDNF-mRFP1-P2A-ECFP, GFAP2.2-EGFP and syn-LSSmOrange at DIV14-16. (a) The amount of released BDNF-mRFP1 was increased when cells were stimulated with $10 \mathrm{mM} \mathrm{KCl}$ or CLTP for $8-10$ days (ordinary one-way ANOVA, $P=0.0034$; significance of stimulated groups to control was assessed by Dunnett's multiple comparisons test $* P \leq 0.05$, $* * * * \mathrm{P} \leq 0.0001$ ). (b) No significant changes in relative BDNF-mRFP1 uptake was found for $10 \mathrm{mM} \mathrm{KCl}$ stimulation. CLTP stimulation significantly reduced BDNF-mRFP1 in the unassigned population and neurons (ordinary one-way ANOVA was used to test for statistical significance within the individual groups (all $P \leq 0.0001)$; significance of stimulated groups to control was assessed by Dunnett's multiple comparisons test $\left.\left({ }^{* * * *} \mathrm{P} \leq 0.0001\right)\right)$. (c) Change in cell type abundance due to the different stimulations. Astrocytes exhibit significantly increased and neurons a slightly decreased abundance, when cultures were treated with cLTP (ordinary one-way ANOVA, $\mathrm{P} \leq 0.0001$; significance of stimulated groups to control was assessed by Dunnett's multiple comparisons test $* * P \leq 0.01, * * * * P \leq 0.0001$ ). (d) When normalized to abundance and total released BDNF-mRFP1 there was no difference in the BDNF-mRFP1 levels in neurons but there was a decrease in astrocytes (ordinary one-way ANOVA, $P \leq 0.0001$; significance of stimulated groups to control was assessed by Dunnett's multiple comparisons test $* * P \leq 0.01, * * * * P \leq 0.0001)$. 
To further investigate a possible influence of BDNF on astrocyte abundance in culture, we treated dissociated hippocampal cultures with $10 \mathrm{mM} \mathrm{KCl}, \mathrm{CLTP}$, TrkB-lgG (a BDNF scavenger), expression of TrkB in neurons by calcium phosphate transfection, application of recombinant BDNF, expression of BDNF-mRFP1 and combined expression of BDNF-mRFP1 with CLTP for 12 days and subsequently quantified average GFAP immunoreactivity (Figure 30). Only BDNF-mRFP1 expression and combined expression with cLTP treatment appeared to have a significant effect, respectively increasing GFAP average immunoreactivity 1.37 or 2.1 -fold.

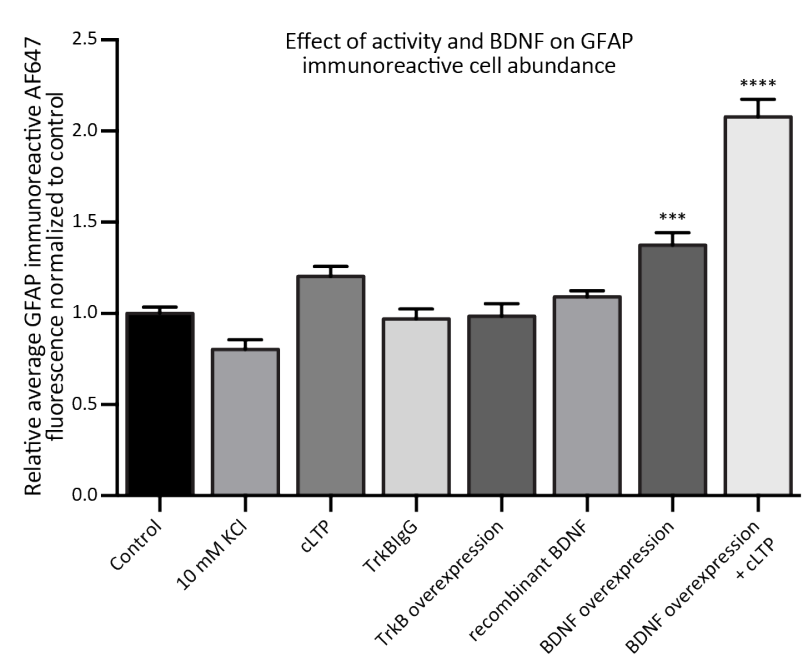

Figure 30: Influence of BDNF abundance on GFAP immunoreactivity. Graph represents averages and SEM for GFAP positive AF647 intensity of $n=15$ images per condition (5 images per coverslip, 3 coverslips per culture). Cultures were treated with $10 \mathrm{mM} \mathrm{KCl}, \mathrm{CLTP}$, TrkB-IgG, overexpression of TrkB in neurons, application of recombinant BDNF, or BDNF-mRFP1 overexpression without and with additional CLTP for 12 days and tested for GFAP immunoreactivity at DIV15, to assess astrocyte abundance. BDNF-mRFP1 overexpression did appear to increase astrocyte abundance and was even more pronounced in combination with cLTP (ordinary oneway ANOVA, $P<0.0001$. Statistical differences of individual groups to control was assessed by Dunnett's multiple comparisons test ${ }^{* * *} \mathrm{P} \leq 0.001$, $* * * * \mathrm{P} \leq 0.0001)$.

In a separate experiment on BDNF-mRFP1-P2A-ECFP, syn-LSSmOrange and GFAP2.2-EGFP expressing cells, we could reduce total released BDNF-mRFP1 in the culture with the application of $0.4 \mu \mathrm{g} / \mathrm{ml}$ TrkB-IgG every 3 days, starting at DIV3 until DIV14-15, by $63.8 \pm 9.1 \%$ (Control $n=19$, TrkB-IgG $n=18$, unpaired two-tailed t test, $p<0.0001$ ). This affected BDNF-mRFP1 uptake by all groups (neurons, astrocytes and unassigned), without significant differences. Neither differential uptake between neurons and astrocytes, nor astrocytic cell abundance was affected. Therefore this data is not shown. 


\section{Discussion}

The overall idea behind this dissertation was to investigate a novel strategy to achieve focal stimulation of neurons, with the ultimate goal to study the influence of activity on the recruitment and release of the neurotrophic factor BDNF. We planned to achieve this by combining optogenetics with an illumination approach inspired by STED/RESOLFT-microscopy. We therefore tested the utility of current optogenetic tools to achieve highly focal depolarization and further examined a proof-ofprinciple of nanoscale activation, using an initial macroscale approach. We also extended the study of BDNF recruitment and release by investigating the cell-to-cell transfer of BDNF to neighboring cells, where we found that BDNF was preferentially taken up by astrocytes and affects the astrocytic population.

\subsection{Nanoscale optogenetics}

In order to gain control over synaptic or even sub-synaptic activity, optogenetics represents a promising approach, especially when studying biological processes in dissociated hippocampal cultures. Under these conditions cells can be easily transfected and are accessible for electrophysiology and light stimulation. However, the most important processing unit of neuronal networks, the synapse, is a very small structure. An average excitatory postsynaptic site in the rodent hippocampus ranges from approximately $0.04 \mu^{2}$ (Schikorski and Stevens, 1997) to approximately $0.1 \mathrm{\mu m}^{3}$ for a dendritic spine (Nimchinsky et al., 2002). The development of a tool allowing control over neuronal activity through the stimulation of single synapses or even smaller synaptic nanodomains, would be very useful to study the synapse-specific mechanisms of synaptic potentiation and depression. This idea is not new; several approaches have been used previously to activate areas in the range of several $\mu \mathrm{m}$ to even small subcellular structures, such as single spines, e.g. by neurotransmitter uncaging (Adams and Tsien, 1993), local pressure application of glutamate, on-cell direct current injection, expression and targeting of chemically and genetically modified receptors and of course also using optogenetic tools, such as light-gated ion-channels (Banghart et al., 2004; Lima and Miesenbock, 2005; Zemelman et al., 2002) or chromophore-assisted light inactivation (Lin et al., 2013). Channels have also been used in combination with genetic engineering, to enrich them at synaptic sites allowing efficient targeted stimulation (Greenberg et al., 2011).

Though Optogenetics with light-gated ion channels is most commonly used to activate populations of cells, attempts have been made to decrease the area of activation by optimization of focal illumination and by making use of two-photon illumination, decreasing out-of-focus activation (Mohanty et al., 2008; Packer et al., 2012; Schoenenberger et al., 2008). A very elegant approach for the physiological and controlled stimulation of postsynaptic sites can be achieved by optogenetic stimulation of the presynaptic neuron. Synapses can then be identified by colocalization of fluorescently labeled PSD-95, to mark post-synaptic sites in the postsynaptic neuron and fluorescently-tagged ChR2 in the presynaptic neuron. This approach has been combined with electrophysiological stimulation in the postsynaptic neuron, demonstrating the usefulness of this 
technique for the study of synaptic plasticity (Zhang and Oertner, 2007). Recent combinatorial expression of spectrally separable light-gated channels with $\mathrm{pH}$-sensitive fluorophores and genetically encoded calcium indicators provides an all-optical approach for multiplex control and tracking of neuronal activity (Li and Tsien, 2012). However, so far no technique exists that allows for the specific and highly focal control of activity in both pre- and postsynaptic structures. Such methods could significantly contribute to our understanding of detailed signaling processes in neuronal networks and the plasticity of individual synapses, the underlying signaling units for complex mechanisms such as the formation of memory.

With this project we aimed to contribute to this endeavor and therefore tested a novel approach using optogenetic tools in combination with an illumination paradigm originally inspired by STED/RESOLFT microscopy, in order to focally control activity in subcellular sites, ideally reducing the area in which light-gated channels open to the size of single synapses or even further to sub-synaptic micro- or nanodomains. The idea is based on the switching property of bi-stable opsins. These lightgated cation-channels can be opened by illumination with one wavelength and closed by illumination of a second (usually red-shifted) wavelength (Berndt et al., 2009). We reasoned, that by activating channels with a center beam and using a partly overlapping donut-shaped surrounding beam for channel deactivation, we would principally be able to reduce channel activation in the outer area, which is simultaneously illuminated by both the activation and inactivation wavelength. Thus we would be able to control the activity of light-gated cation-channels in a highly focal area, eventually even beyond the diffraction limit of light.

\section{Functional characterization and testing of channels}

The approach described above critically depends on the property of light-gated channels to close when simultaneously illuminated with both activation and inactivation wavelengths of light. In order to identify suitable candidates, exhibiting the required photocurrent reduction for simultaneous illumination, we tested a battery of diverse channel candidates. We focused our investigation on the step-function opsins, since they appeared most promising given their switchable gating properties.

Dissociated hippocampal cultures were transfected using a standard calcium phosphate transfection protocol with expression constructs for CMV, synapsin or CaMKIl $\alpha$ promotor controlled expression of EYFP or mCherry tagged light-gated ion-channels/pumps. Functional validation of the expressed channels was performed using patch-clamp recordings of cells identified by their respective fluorescence. A tendency towards intracellular accumulations of fluorescence was detected for mCherry, but not for EYFP expressing cells. Such accumulations can result from overexpression, but they had no obvious influence on channel function. If accumulations should become a problem for focal activation experiments, a possible exchange of the mCherry fluorophore can be considered. Photocurrents could not be evoked from cells, where no expression was visually detected. 
Channel specific light evoked photocurrents were then tested qualitatively to determine channel activation spectra, conductance, gating kinetics and the ability to close channels with a second wavelength. Individual variations in transfection efficiency and expression levels pose a challenge for comparison of recordings from different cells. We recorded photocurrents from single cells evoked by the illumination with multiple sequential wavelengths to compare the responses. To avoid signal masking by spontaneous neuronal activity and to single out photocurrents from the transfected light-gated ion-channels, spontaneous activity was suppressed by the application of TTX, APV, CNQX and gabazine, blocking action potentials, NMDA channels, AMPA channels, and GABAergic transmission, respectively (Nikolic et al., 2009). We could successfully record light-evoked photocurrents from cells up to DIV30 and photocurrents were similar to those reported in the literature (Boyden et al., 2005; Nagel et al., 2003). As expected, photocurrents of fast photocycle variants (ChR2 H134R, T159C, E122T/T259C, L132C) were generally strong and disappeared quickly when illumination was stopped. This group of channels did not exhibit interference of the conducting state for any of the simultaneous illumination wavelengths tested. Though it is assumed, that the tested channels share the fundamentally same photocycle, opening and traversing through similar intermediate channel states and only differ in ion-specificity, conductance, activity spectrum and channel kinetics. This cannot confidentially be inferred for the photocycles of the C1V1, ReaChR, Chronos and CoChR channels, because their photocycles have not been as extensively studied. However, the absence of interference with the conducting state by simultaneous illumination with other wavelengths, indicates that these channels have a comparable and fast photocycle with strong single channel conductance. Most channels of this group displayed pronounced peak to plateau current ratios.

For fast photocycle channels (Figure 3), initially all channels are closed and open at once upon illumination. Subsequently channels proceed through their photocycles, close and re-open. They finally reach an equilibrium (the stationary current phase) of constantly opening and closing channels, in which approximately $50 \%$ of all illuminated channels can be found in the open-channel state (Feldbauer et al., 2009), inferred by the peak to stationary current ratios. The stationary current is very robust; the individual contribution of a single channel is very small and only of a short persistence, because each individual channel closes quickly and re-opens again. Therefore illumination with a closing wavelength, exciting the conducting P520 or respective state (Figure 1), accelerating channel closure, will have only a minor influence on the total photocurrent of fast photocycle channels. Hence we extended our tests to bi-stable step function opsins (SFOs) and slow photocycle variants, which we generated by mutagenesis for variants that were not yet published. These channels could be reliably opened for extended times by a single activation wavelength pulse. In the case of the original published stable step-function opsin (SSFO) variant, we recorded a steady photocurrent from channels that remained open for at least $10 \mathrm{~min}$ and open times of $30 \mathrm{~min}$ or longer have been reported (Yizhar et al., 2011). During the functional assessment of these channels, we noticed that SFOs appear to be much more sensitive to light, than the original ChR2. Even broad- 
spectrum daylight at standard room intensities and light from the bright-field light source of the microscope was sufficient to gate channels. When fast photocycle channels were exposed to the bright-field light source, evoked photocurrents were only a fraction of the maximal currents evoked by $488 \mathrm{~nm}$ light exposure. But, because of the prolonged open channel state of SFOs, even if only a small fraction of channels is activated, they start accumulating in the open channel state. Therefore cells expressing these channels should be kept in completely dark conditions, to avoid unwanted channel stimulation. Repeated excitation of ChR2 expressing brain slices for up to 4 days does not lead to cytotoxicity (Schoenenberger et al., 2009), but less exposure may still cause excessive $\mathrm{Ca}^{2+}$ influx through VGCCs and thus have an effect on cell physiology. In addition it has been reported that, because of their significantly slower photocycles, the ChR2 C128 mutant SFO variants (C128A, C128T and C128S) tend to accumulate in a separate previously undetected branch of the photocycle, especially after prolonged and repeated activation. This branch occurs at a late stage of the photocycle, either via the late-conducting (P520) or closed channel state (P480) and leads to P390, a new non-conducting state (Figure 1) (Schoenenberger et al., 2009; Stehfest et al., 2010). Because they share the same photocycle, this branch is also expected to exist for the other related ChR2 variants. Even though no significant photocurrent decrease for prolonged and repeated stimulation of other variants has been observed and most likely only few channels end up in this non-conducting state, either because of their short persistence in the late photocycle stages or the time constants for the transitions from P390 to the P470 ground state, which might be significantly lower than those of ChR2 C128 mutant variants. It is important to note that closing of C128 variants within 3 seconds after opening was reported to reduce the photocurrent decline already from $66.5 \%$ to $16.7 \%$ by bypassing the branch-point to the non-conducting branch (Schoenenberger et al., 2009). Thus the ChR2 C128 variants may retain switching properties and remain useful for focal activation, if they are not used for extensively long stimulations. Indeed, in our tests of the C128A variant, where channels activated by $488 \mathrm{~nm}$ light were closed after 6.5 seconds, photocurrent decreases were not observed, except when illuminated at $405 \mathrm{~nm}$ (Figure 5). The photocurrent decrease following $405 \mathrm{~nm}$ activation was most likely caused by either insufficient channel closure at the lower $594 \mathrm{~nm}$ intensities or $405 \mathrm{~nm}$ stimulation significantly increases the chances for driving channels into a nonconducting state, a potentially interesting observation, that could be addressed in future studies. Whether other channel variants containing the corresponding C128 mutation also possess such a non-conducting state is not clear. This behavior has not been addressed during our experiments and has so far not been reported in the literature.

An additional characteristic of SFO channels is that channel closing is considerably slower than opening. Since a focal stimulation approach relies on closing all channels quickly with coillumination, control over channel closure is of paramount importance. Therefore the influence of different intensities and exposure times of light was tested. Closing was effectively accelerated with higher intensities of $594 \mathrm{~nm}$ illumination (or $405 \mathrm{~nm}$ in the case of the C1V1 slow mutant). The ChR2 variants C128S/H134R/T159C and C128S/L132C/T159C, the two CoChR mutants C108S and 
C108S/D136A and the C1V1 E122T/C167S channels all showed promising results in terms of channel closing with coillumination of a second wavelength during initial screening. The C1V1 mutant exhibited photocurrent reductions of up to $84 \%$. Interestingly, this channel exhibited brief photocurrent increases at the onset and offset of $405 \mathrm{~nm}$ light when simultaneously illuminated with $488 \mathrm{~nm}$ light. The photocycle of this C1V1 variant has not been as thoroughly studied as ChR2 (Prigge et al., 2012), therefore it not easy to make sophisticated assumptions. This unexpected behavior, we observed can most likely be explained by the slow closing kinetics of the channel. Illumination with $405 \mathrm{~nm}$ light short-circuits the otherwise longer traverse through the closed intermediate states, thus slightly increasing the number of channels in the open state. But we cannot exclude the existence of multiple further conducting states, that branch off from the open-channel state. The ChR2 L132C SFO variant exhibited in part much slower closing than other SFO variants and was therefore not considered useful for our purpose. Even though $561 \mathrm{~nm}$ illumination of the ChR2 SFO variant channels should be more efficient in switching channels from the P520 state to the closed state, it partly cross-activates the P470 state and therefore also opens channels at the same time, mitigating the photocurrent reduction. In contrast, $594 \mathrm{~nm}$ illumination appeared to stimulate the P520 state and subsequent channel closure strongly, without significant cross-stimulation of P470.

For initial identification of suitable light-gated channel candidates, we used wide-field illumination with the $40 x$ objective at power densities of $405 \mathrm{~nm} 86.83 \mathrm{~mW} / \mathrm{cm}^{2}, 488 \mathrm{~nm}$ $295.6 \mathrm{~mW} / \mathrm{cm}^{2}, 561 \mathrm{~nm} 146.3 \mathrm{~mW} / \mathrm{cm}^{2}$ and $594 \mathrm{~nm}$ for ROE photomask \#1 of $801.48 \mathrm{~mW} / \mathrm{cm}^{2}$ and $\# 15$ - 19 of $1.08 \mathrm{~W} / \mathrm{cm}^{2}$. $594 \mathrm{~nm}$ intensity was directly related to the efficiency of suppressing depolarizing currents. Given only partial reduction of photocurrents by coillumination with $594 \mathrm{~nm}$ light, we reasoned that the ratio of inactivation to activation power was not sufficient and therefore modified parts of the light path to shift more transmittance to the DL594, which only led to minor reductions in the wide-field illumination power densities using the $40 x$ objective of $405 \mathrm{~nm}$ $27.10-64.56 \mathrm{~mW} / \mathrm{cm}^{2}, 488 \mathrm{~nm} 228.1 \mathrm{~mW} / \mathrm{cm}^{2}, 561 \mathrm{~nm} 133.4 \mathrm{~mW} / \mathrm{cm}^{2}$, but markedly increased the available $594 \mathrm{~nm}$ power densities for the $40 x$ objective ROE photomask $\# 1$ : $129.75 \mathrm{~W} / \mathrm{cm}^{2}$ and \#15 - 19: $267.25 \mathrm{~W} / \mathrm{cm}^{2}$. Because the $594 \mathrm{~nm}$ photomasks did not cover the whole area illuminated with wide-field illumination, the FRAP-photomasks were used for stimulation of $405 \mathrm{~nm}$ with $9.92 \mathrm{~W} / \mathrm{cm}^{2}$ and $488 \mathrm{~nm}$ with $41.92 \mathrm{~W} / \mathrm{cm}^{2}$. The power-densities for illumination with the FRAPphotomask were slightly underestimated. Later laser intensity measurements showed that FRAP photomask intensities are higher than the corresponding laser power settings of the wide-field TIRF laser. However, this configuration allowed us to test the channels at an inactivation to activation power ratio of at least 3:1. At these intensities no immediate negative effects on cell appearance or recorded photocurrents was detected. We focused on the C128A mutation channel variants, because of their switching properties (Berndt et al., 2009; Prakash et al., 2012). However, in our experiments with C128A/H134R/T159C using FRAP-stimulation and ROE photomask \#1 for $594 \mathrm{~nm}$ inactivation, we were only able to reduce $488 \mathrm{~nm}$ elicited photocurrents by about $54 \%$ at which point photocurrents plateaued. We were able to increase the photocurrent reduction even further by 
decreasing the $488 \mathrm{~nm}$ power density to $4.48 \mathrm{~W} / \mathrm{cm}^{2}$, but still did not reach anything close to $100 \%$ photocurrent reduction. Information in the literature implies that $594 \mathrm{~nm}$ inactivating light should be roughly 1000 -fold more intense than $488 \mathrm{~nm}$ activating light to achieve photocurrent-inactivation of about $95 \%$ for ChR2 C128S (Venkatachalam and Cohen, 2014). This information was not available at the time we conducted our experiments, but provides a good reference for trouble-shooting and is indeed very helpful for the design of future experiments, promising that much higher photocurrent reduction efficiencies using $488 \mathrm{~nm}$ than the ones we observed can be achieved. For this reason the use of a C128S mutant alone should eventually be considered, because the additional H134R/T159C mutations in our C128S/H134R/T159C channel variant did not improve photocurrents as dramatically as expected. It would also be interesting to test the sdChR C138S/E154A variant, because it has been reported to possess good photocurrent inhibition (Venkatachalam and Cohen, 2014) or to reduce $488 \mathrm{~nm}$ laser intensities further. In our experiments, we tested the lowest available laser power (1\%; $4.48 \mathrm{~W} / \mathrm{cm}^{2}$ ) for the FRAP-photoactivation mask, but further power density reduction could be attained by using other filters or beam splitters. Theoretically, wide-field $488 \mathrm{~nm}$ stimulation with power densities down to $106.91 \mathrm{~mW} / \mathrm{cm}^{2}$ is possible. This would bring us to 1000 -fold higher $594 \mathrm{~nm}$ power densities, but may also recruit channels outside of the area covered by $594 \mathrm{~nm}$ illumination. On the other hand, we found activation with $405 \mathrm{~nm}$ to be more useful than $488 \mathrm{~nm}$. $405 \mathrm{~nm}$ did not cross-interfere with the P520 state as $488 \mathrm{~nm}$ illumination did, as evident by brief current transients when $488 \mathrm{~nm}$ illumination is stopped (Figure 6) (Venkatachalam and Cohen, 2014). These small stimulation artifacts may mask photocurrent signal when operating at nanoscale dimensions. With $405 \mathrm{~nm}$ illumination we did not observe these current increases upon illumination stop. The photocurrent strength for $405 \mathrm{~nm}$ activation was comparable to that at $488 \mathrm{~nm}$, even though $405 \mathrm{~nm}$ power densities were significantly lower. Channels were more efficiently inactivated by $594 \mathrm{~nm}$ light following $405 \mathrm{~nm}$ activation, than following $488 \mathrm{~nm}$ activation, even at only roughly 100-fold higher $594 \mathrm{~nm}$ power densities. It is not completely clear why this is the case. We assume that $405 \mathrm{~nm}$ illumination activates the P470 open state, but because of the lower stimulation efficiency and used power densities these currents can be better reduced than $488 \mathrm{~nm}$-induced photocurrents. Another possibility is that the channels actually have a separate conducting state for light stimulation in the UV spectrum, which is more easily reduced by $594 \mathrm{~nm}$ illumination. In any case, $405 \mathrm{~nm}$ activation provides an interesting alternative.

In addition to ChR2 C128S/H134R/T159C, we also tested inactivation of the two CoChR slow C108S and C108S/D136A photocycle SFO variants. In accordance with our earlier tests and what is reported (Venkatachalam and Cohen, 2014), we were not able to completely reduce $488 \mathrm{~nm}$ evoked currents with $594 \mathrm{~nm}$ coillumination. The double mutant exhibited less $488 \mathrm{~nm}$ induced cross-talk to the P520 state than $405 \mathrm{~nm}$ illumination, inferred from the absence of brief transients at offset; 405 $\mathrm{nm}$ evoked currents could be reduced with $594 \mathrm{~nm}$ coillumination and did not exhibit transients at light offset. Under similar conditions we found that ChR2 C128A/H134R/T159C photocurrents were slightly stronger than those of the CoChR SFO variants and would therefore be favored, however this 
can also be an effect of different expression levels. Furthermore the C1V1 E122T/C167S SFO variant, for which $561 \mathrm{~nm}$ mediated photocurrents were reduced $84 \%$ with $405 \mathrm{~nm}$ coillumination, is also worth being considered for future experiments.

We also tested the effect of increased $594 \mathrm{~nm}$ power densities on channel closure of the ChR2 T159C and CoChR fast-photocycle variants, because we were curious if we could detect any photocurrent reduction. In fact we found that due to substantial cross-activation of the P470 state by the stronger $594 \mathrm{~nm}$ intensities, we could now observe pronounced $594 \mathrm{~nm}$ activation of channels (Figure 7). Interestingly simultaneous $594 \mathrm{~nm}$ illumination with 488 or $405 \mathrm{~nm}$ only led to brief current transients or a weak photocurrent increase. This coillumination experiment demonstrates that $594 \mathrm{~nm}$ illumination can also close the channels, but $594 \mathrm{~nm}$ illumination might accelerate the photocycle by bypassing the inactivity states and increasing the number of channels in the open state.

In addition to the ChR-only approach, we also tested the feasibility of the eNPAC construct, through which blue light illumination causes ChR2-mediated depolarization and orange - red light illumination causes NpHR3.0-mediated hyperpolarization. Unlike the photocurrent reduction approach, to open and close a single channel type with two different wavelengths, as described above, here we tested whether it is principally possible to use a second light-evoked counterbalancing photocurrent, caused by the activation of NpHR3.0, to annihilate ChR2-mediated depolarization in an area of simultaneous illumination. ChR2 was easily gated in cells expressing eNPAC using $488 \mathrm{~nm}$ illumination at $86.83 \mathrm{~mW} / \mathrm{cm}^{2}$. Photocurrents were markedly smaller than for the single ChR2 H134R, most likely, because of differential expression levels of ChR2 H134R and eNPAC. Besides the substantially larger size of the eNPAC construct, it was also expressed using the human synapsin promotor while ChR2 H134R was expressed using the CaMKIl $\alpha$ promotor. Comparable magnitudes of NpHR3.0 hyperpolarizing photocurrents were recorded at $561 \mathrm{~nm}$ at $146.3 \mathrm{~mW} / \mathrm{cm}^{2}$ and $594 \mathrm{~nm}$ at approximately $160.3 \mathrm{~mW} / \mathrm{cm}^{2}$. Such evoked hyperpolarizing currents where already strong enough to completely counterbalance ChR2-mediated depolarization and even shift cells to a slightly hyperpolarized state (Figure 4). As we did show during the following experiments, such an approach is principally possible. Hyperpolarizing currents are easily tuned by adjusting $594 \mathrm{~nm}$ laser power and can be adjusted to be stronger or weaker than the ChR2 mediated depolarizing currents (Figure 9). For our experiments, we determined a range of $80.15-160.3 \mathrm{~mW} / \mathrm{cm}^{2} 594 \mathrm{~nm}$ illumination sufficient to completely counterbalance ChR2-mediated depolarization. These values fluctuated between different experiments, but these fluctuations should be minimal, because of the approximate equimolar expression of ChR2 and NpHR3.0 by using a P2A sequence construct. As mentioned earlier, ChR2 activation using $405 \mathrm{~nm}$ is preferred to $488 \mathrm{~nm}$ activation, to reduce peak to stationary current ratios and avoid currents that arise from NpHR3.0 blue-light coactivation.

With their fast on- and offset, ion-pumps have ideal properties for temporally precise activation and reduction of ChR2-mediated photocurrents. This feature is an advantage over single 
step function light-gated channels, which have comparably slower kinetics. However, the development of future optogenetic tools may yield single channels with faster switching kinetics that allow depolarization and hyperpolarization. For instance, the ion selectivity of ChR2 has recently been selectively modified to create a chloride-conduction channel (Berndt et al., 2014; Wietek et al., 2014) and stronger chloride-pumps have already been generated (Chuong et al., 2014). Despite our promising results and the fact that the net voltage change caused by simultaneously activated ChR2 and NpHR3.0 can be kept at $0 \mathrm{mV}$, one has to acknowledge, that there will still be changes in ion concentration. This approach might be useful to study local voltage-dependent processes, but only if an influence of the different ion concentrations on the observed mechanism can be excluded. Fortunately most ChR2 variants do not exhibit $\mathrm{Ca}^{2+}$-permeability, which reduces the risk of mediating unwanted physiological effects, caused by the cation-influx. However, data exists suggesting that even $\mathrm{Cl}^{-}$ions may function as second messengers, tuning $\mathrm{GABA}_{A}$-receptor responses (Succol et al., 2012). This possibility should be investigated thoroughly before this technique can be applied. Alternative tools for hyperpolarization, like the hyperpolarizing proton-pumps, termed archaerhopospins (Arch), found in Halorubrum sodomense and ArchT (Chow et al., 2010; Han et al., 2011), have already been used successfully in neurons. An intriguing feature of the use of constructs like eNPAC, that mediate depolarization and hyperpolarization, is that it can also be "inverted" and thus, used not only to for focal depolarization, but also to create highly focal areas of hyperpolarization, potentially mimicking inhibitory synaptic input.

\section{Electrophysiological and visual detection of focal stimulation}

Consistent focal stimulation relies on equal distribution of light-gated channels in cell membranes and a slow rate of channel diffusion, so that activated channels remain confined to the site of illumination. We further examined ChR2, SSFO and NpHR3.0 distribution in the membrane of expressing neurons using conventional epifluorescence microscopy and STED microscopy. In addition, SSFO diffusion was assessed by fluorescence-recovery-after-photobleaching (FRAP) experiments to determine channel diffusion speed. Fluorescently tagged ChR2 variants were homogeneously distributed over the membrane and only the NpHR3.0 variant showed some grainy patterns, when imaged using STED microscopy, suggesting that it may form small clusters. Nevertheless, the distribution of these clusters appeared homogeneous. The lateral diffusion coefficient for ChR2 was $3-9 \times 10^{-2} \mu \mathrm{m}^{2} / \mathrm{s}$, with minor differences in the attached EYFP or mCherry fluorophore, the tested membrane area, or neuronal process versus cell soma.

To provide a proof-of-principle for nanoscale activation, we tested if highly focal light-gated channel activity can be detected using electrophysiological or calcium imaging. Using the DirectFRAP laser we stimulated ChR2 T259C channels on the cell body. In the smallest areas tested $\left(1.13 \mu \mathrm{m}^{2}\right)$, we found that evoked currents were almost indistinguishable from noise. Further reductions to areas comparable to the size of a synapse in the rodent hippocampus of around $0.04 \mu \mathrm{m}^{2}$ (Schikorski and Stevens, 1997), will likely impose a problem for the detection of the signal electrophysiologically, 
although averaging of multiple repeated stimulations may improve detection in smaller areas. An alternative approach, necessary to verify focal activation, is visual confirmation of channel activation using voltage or calcium-sensitive dyes. Initially we investigated the use of the commonly used acetoxymethyl ester bound Fluo-4 calcium dye and the latest generation of genetically encoded calcium indicators; GCaMP5G and 6s (Akerboom et al., 2012; Chen et al., 2013; Gee et al., 2000). Although these calcium indicators have the highest reported signal to noise ratios, they are not ideal, due to the similar illumination spectrum for calcium imaging and channel activation. If cells are imaged with wide-field epifluorescence microscopy or related techniques, $488 \mathrm{~nm}$ illumination will also activate channels. We reasoned that eventually channels with red-shifted activation spectra could be used and thus extended our analysis to ReaChR and C1V1 channels. Unfortunately until now there is no such thing as a channel exclusively activated by the red spectrum and all tested channels still possessed strong activation in the blue spectrum. Because of the superb photocurrent inactivation that we observed for the C1V1 E122T/C167S using $405 \mathrm{~nm}$, it may be possible to illuminate the whole-field with the closing wavelength, while exciting green calcium indicators with $488 \mathrm{~nm}$, subsequently focally activating a small area with $488 \mathrm{~nm}$ light by removing the $405 \mathrm{~nm}$ illumination there. This is however a very complicated approach. The fact, that full-field optogenetic controlled stimulation, while imaging a green calcium indicator is principally possible, has recently been shown (Venkatachalam and Cohen, 2014), but it remains to be tested in combination with a focal stimulation approach.

Finally, we directed our attention to newly developed genetically encoded red calcium indicators and tested the use of RCaMP1c (Akerboom et al., 2013), which would allow visualization of calcium indicators excited by wavelengths that do not activate the ChR2 based channels, which are activated by blue light. RCaMP1c was expressed along with a ChR2 L132C in hippocampal neurons and an area of $4.91 \mu \mathrm{m}^{2}$ was stimulated with $488 \mathrm{~nm}$ by using the DirectFRAP and RCaMP1c was imaged with $561 \mathrm{~nm}$ whole-field illumination at low intensities. The L132C channel was chosen, because it exhibits generally strong photocurrents, does not exhibit particularly strong activation by $561 \mathrm{~nm}$ light and is reported to allow permeation of $\mathrm{Ca}^{2+}$ and therefore its activation would be more easily detected by calcium indicators. However, for small areas of $4.91 \mu^{2}$ it was difficult to detect a significant rise in RCaMP1c fluorescence due to channel activation. Small fluorescence increases that coincided with photostimulation were evident, but they were difficult to distinguish from background signal in single traces. Repeated stimulation and averaging of signal may improve detection in this case. Voltage-indicator dyes might present a more suitable tool for the detection of light-gated channel mediated depolarizations of small areas. And indeed optical stimulation has already been used successfully along with voltage imaging (Hochbaum et al., 2014). Although the signal to noise of fluorescent voltage-sensitive dyes is often not as high as for calcium indicators and still possess a substantial spectral overlap with the ChR2 activation spectrum. Even though recent archaerhodopsin-based voltage indicators are reported to allow detection of miniature excitatory postsynaptic depolarizations using near infrared light (Hochbaum et al., 2014). We tested these 
indicators, but failed to detect local ChR2 mediated depolarizations, possibly due to low responses. An advantage of these indicators is their far-red activation spectrum, which would not interfere with ChR2 activation, but the required light intensities for imaging are currently very high and fluorescence signal increases relatively small and potentially not high enough for use of detection of focal activation.

This leads to the question of whether current optogenetic tools are adequate to be used for a simultaneous illumination approach in order to achieve focal activation. Using macroscopic photocurrent from recordings of Xenopus oocytes, available information about the ChR2 channel kinetics and assuming similar expression and abundance as for bacteriorhodopsin, open channel populations of ChR2 are assumed to be $10 \%$ and single channel conductance of wildtype ChR2 is estimated to be around $50 \mathrm{fS}$, which yields single channel currents of around $5 \mathrm{fA}$ (Nagel et al., 2003). Using nonstationary noise analysis, conductances of wildtype ChR2 and ChR2 H134R in HEK cells were $41.5 \mathrm{fS}$ for single channel $\mathrm{Na}^{+}$conductance at $200 \mathrm{mM}$ extracellular $\mathrm{Na}^{+}$, with single channel currents of $3.5 \mathrm{fA}$ at $-60 \mathrm{mV}$ (Feldbauer et al., 2009). It was also shown, that in the stationary phase around $51 \%$ of the channels are in the open channel state, thus approximately 226 channels are active per $\mu \mathrm{m}^{2}$ cell membrane in HEK cells (Feldbauer et al., 2009). However, H134R exhibits stronger stationary photocurrents, because of an extended persistence in the open channel state, thus $60 \%$ of the channels are expected to be open during stimulation (Feldbauer et al., 2009; Nagel et al., 2005a). If the proposed channel density in HEK cells roughly translates to a similar presence in neuronal membranes a stimulation of a synaptic size area $\left(0.04 \mu \mathrm{m}^{2}\right)$ would lead to the opening of around 9 channels and currents of roughly $31.5-45 \mathrm{fA}$ at $-60 \mathrm{mV}$. This is too low to be detected using standard electrophysiological methods. In our experiments the electrical noise level was about 8 pA, therefore such a weak signal cannot be detected, especially if the stimulated site is further away from the recording electrode. Other studies using nonstationary noise analysis estimate ChR2 single channel conductance at around $1 \mathrm{pS}$, which principally would allow single channel recordings (Lin et al., 2009). However, the absence of such published recordings to date suggest that this value may be an overestimation and that the previous values above are in fact more realistic. It is unfortunate, that ChRs have not evolved as high conductance optogenetic tools for scientists to study and manipulate molecular and cellular processes in one of the most complex organs, but simply to guide microalgae towards or away from light, thus optimizing the light conditions for photosynthetic growth. Naturally, these channels need to allow organisms to sense different light intensities over several orders of magnitude and prevent complete block by low light saturated depolarization and dangerous deflections from physiological intracellular ion-concentrations, that might result from higher conductance channels. Thus the channels have evolved to transport a minimal amount of ions and further decrease this transport under conditions of extended illumination to relatively small stationary levels (Hegemann and Moglich, 2011). Increased laser intensities will not lead to stronger photocurrents after channels have been activated, but rather lead to a loss of spatial selectivity by spatial tails of the laser beam and scattered light as well as stimulation of channels in out-of-focus 
membrane (Dobrucki et al., 2007; Schoenenberger et al., 2011). This is suboptimal for local stimulation of subcellular structures. Ideally a center two-photon activation could be used to restrict ChR2 activation to very small volumes and subcellular structures with high spatial selectivity, decreasing the chance of out-of-focus membrane depolarization (Packer et al., 2012; Rickgauer and Tank, 2009).

To make light-gated channels usable for highly focal stimulation, further improvements are needed and we either have to increase channel abundance in the membrane area to be stimulated, e.g. by higher expression levels or targeting motifs to synapses, or single channel conductance has to be further improved to match the one of native neuronal ion-channels. In order to mimic synaptic activation in physiological conditions, ideal channels would possess properties that match the mixed currents elicited by AMPA and NMDA receptors at excitatory synapses. The largest proportion of glutamatergic synapses, approximately $70 \%$, contain both AMPA and NMDA receptors, $20 \%$ only AMPA and the rest only NMDA (Bekkers and Stevens, 1989). Amounts of NMDA receptors are reported to be mostly consistent and independent of spine volume and synapse location, however AMPA receptor densities have been described to vary in abundance depending on their location on the dendritic tree (Andrasfalvy and Magee, 2001). Thus for an ideal imaginary excitatory synaptic site, we can assume that synaptic transmission at low frequencies is conveyed by 5.9-35 AMPA receptors and between 0.5-19 NMDA receptors, of which during the peak of the postsynaptic current only $1-2$ receptors are expected to be open at the same time (Nimchinsky et al., 2004; Spruston et al., 1995). Single channel AMPA conductances have been determined to be around 8 - 10 pS (Jonas and Sakmann, 1992; Spruston et al., 1995). And NMDA receptors have a conductance of about 45 pS (Gibb and Colquhoun, 1992; Spruston et al., 1995) of which approximately $10 \%$ is contributed to by calcium conductance at an extracellular calcium concentration of $2 \mathrm{mM} \mathrm{Ca}^{2+}$ (Jahr and Stevens, 1993). To match currents of a single AMPA receptor, the conductance of a wildtype ChR2 needs to be enhanced roughly 200-fold and to match a single NMDA receptor, roughly $1000-$ fold, including a $10 \% \mathrm{Ca}^{2+}$ conductance. Recently developed light-gated ionotropic glutamate channels could potentially help to overcome such problems (Berlin et al., 2016; Volgraf et al., 2006). For the presynaptic site it is much harder to define clear limits of stimulation, because presynaptic stimulation is normally preceded by an action potential and strong depolarization.

Many promising improvements have been made to light-gated channels to optimize channel conductances, but still to date the channel producing the strongest photocurrent that we could find, was CoChR, which had photocurrents roughly 10-fold higher than for the standard ChR2 H134R. But these fast photocycle variants are not actually useful to achieve depolarization at the nanoscale, because they lack the inactivation properties of the slow photocycle variants. The slow photocycle variants, on the other hand, have significantly reduced photocurrents compared to the fast photocycle variants. Activation at the nanoscale will require new tool development to overcome these current limitations. 


\subsection{BDNF}

BDNF is an intriguing protein; although small and of general low abundance in the brain, it is one of the key mediators and regulators of neuronal differentiation, growth and formation of neuronal processes and connections, and synaptic plasticity, which shows that it is fundamentally involved in almost all essential processes, that characterize the brain. BDNF is essential for long-term potentiation of synaptic strength, which is thought to be the mechanism underlying learning and memory. The potentiation of synaptic strength occurs in a synapse-specific manner at synapses in which there is a coincident increase in pre- and post-synaptic activity. We hypothesized that BDNF may be specifically targeted to synapses with increased activity, to promote long-term potentiation of their strength. We aimed to address the question of activity-dependent recruitment of BDNF by imaging trafficking mRFP1-tagged BDNF combined with focal optogenetic stimulation as described above. These experiments led to the observation that BDNF-mRFP1 was transferred to neighboring cells.

\section{Influence of focal light stimulation on BDNF recruitment}

Previous work using fluorescently tagged BDNF in rat neuronal cultures already demonstrated that BDNF is localized to secretory granules and can be found at synaptic junctions (Haubensak et al., 1998). In later studies it was shown that BDNF localized to pre- and or postsynaptic sites in response to glycine-induced increase of neuronal network activity (Dean et al., 2012) and that it can be released from pre- and postsynaptic sites in response to neuronal activity (Kohara et al., 2001; Kuczewski et al., 2008). Neurotrophin-containing DCVs can fuse and release their cargo at synaptic and extra-synaptic sites in an activity-dependent fashion (Farina et al., 2015; Matsuda et al., 2009; van de Bospoort et al., 2012). In addition, BDNF containing vesicles are highly mobile and actively traverse axons and dendrites. This prompted the hypothesis that BDNF-containing DCVs may be recruited to sites of increased activity. In timelapse-imaging experiments with BDNF-mRFP1 expressing neuronal cultures, we observed highly mobile fluorescent puncta, actively moving in and out of the cell body and perambulating neuronal processes, as previously described (Dean et al., 2012). Using the focal optogenetic stimulation approach described above (with a single activating wavelength), we co-expressed BDNF-mRFP1 with a ECFP labeled ChR2, either T159C or L132C, connected via a P2A sequence. By performing patch-clamp electrophysiological recordings from expressing cells in the whole-cell configuration we could detect light-evoked currents in response to 405 and $488 \mathrm{~nm}$ illumination. These currents were not present in ECFP negative cells. As a stimulation protocol, we used $3 \mathrm{~min}$ long stimulations at $0.5 \mathrm{~Hz}$ and $250 \mathrm{~ms}$ pulses, to test the effect of focal 405 and $488 \mathrm{~nm}$ illumination at power densities of 300 and $1,300 \mathrm{~W} / \mathrm{cm}^{2}$ using the \#10 photoactivationmask of the DirectFRAP system for the illumination of a $112.36 \mu \mathrm{m}^{2}$ large spot on randomly chosen processes that were positive for BDNF-mRFP1 and ECFP. As described earlier, laser powers of the DirectFRAP were initially underestimated due to the relatively low powers of the corresponding TIRF laser at the given settings. $488 \mathrm{~nm}$ illumination bleached the mRFP1 signal and 
was therefore not used, because it did not allow for the tracking of fluorescent puncta during the stimulation. $405 \mathrm{~nm}$ laser stimulation, on the other hand, did not bleach the mRFP1 fluorophore. We mainly focused on the use of the $\mathrm{L} 132 \mathrm{C}$ variant, because of its presumptive $\mathrm{Ca}^{2+}$-permeability; but we found, that focal stimulation of both ChR2 T159C and L132C led to pronounced accumulation of fluorescent BDNF-mRFP1 puncta in the area of activation. Before, during, and after stimulation, BDNF-mRFP1 puncta actively moved through the illuminated area, indicating that neuronal processes and microtubule transport in the illuminated region remained intact. The accumulation of BDNFmRFP1 puncta at the site of stimulation began during the stimulation and continued for up to $90 \mathrm{~min}$ after stimulation, after which it started to disappear. However, accumulations could also be seen in the control group of neurons expressing BDNF-mRFP1 alone without ChR2 present. Although weaker and occurring less often, this suggests that the effect of BDNF-mRFP1 vesicle accumulation is at least in part mediated by the light exposure. There was no obvious evidence that the laser illumination was leading to damage or loss of membrane integrity. Direct laser exposure has been reported before to cause depolarization in neurons without obvious damage occurring (Fork, 1971; Hirase et al., 2002; Hirase et al., 2012). Such currents might also here be responsible for the stopping of the BDNF puncta, especially if the depolarizing currents also entailed a rise of the $\mathrm{Ca}^{2+}$-concentration. Another possibility would be photodamage by heat or by direct interaction with the proteins and intracellular structures involved in DCV transport (microtubuli, motor proteins); also fluorophore excitation created reactive oxygen species (Jay, 1988; McLean et al., 2009) could affect BDNF-mRFP1 transport. Such mechanisms are indeed used purposefully to inactivate the function of genetically modified proteins locally (Jay, 1988; Lin et al., 2013; McLean et al., 2009). Our protocol exposed the neuronal process for a total of $22.5 \mathrm{~s}$ with $405 \mathrm{~nm}$ at $3 \mathrm{~W} / \mathrm{mm}^{2}$, which translates to an energy dose of $67.5 \mathrm{~J}$ for the same area. For fluorophore/chromophore assisted light inactivation longer exposures and higher laser powers are normally used (Lin et al., 2013; McLean et al., 2009). Nevertheless, it is possible that the $405 \mathrm{~nm}$ exposure led to the generation of reactive oxygen species by excitation of the ECFP fluorophore attached to the ChR2, even though ECFP is not an ideal candidate for fluorophore assisted light inactivation (McLean et al., 2009). This might have affected the cell membrane integrity, but is unlikely to affect the DCV transport mechanism itself, due to the small effective distance of fluorophore assisted light inactivation. It is important to note, that not only the BDNF-mRFP1 harboring vesicles that were directly exposed to the laser light accumulated, but vesicles that traversed the area after stimulation were also recruited. Moreover, this accumulation started to disappear again after $90 \mathrm{~min}$, which excludes permanent photodamage to the intracellular cytoskeletal elements and leaves strong local depolarization as a consequence of channel activation and generation of free radicals, affecting membrane integrity, as the most reasonable explanation for accumulation of BDNF vesicles at the site of stimulation. For this reason, these experiments were discontinued, but could eventually be resumed at weaker light intensities or focusing on larger subcellular areas to decrease the chance of damage, mediated either by direct light exposure, the generation of reactive oxygen species or depolarization that is caused by direct light exposure. It 
would also be interesting to distinguish between pre- and postsynaptic and extra-synaptic sites, e.g. by the expression of fluorescently synaptic markers. Because the BDNF-mRFP1 and the ChR2 are coexpressed from a single plasmid with a P2A sequence separating them, the ECFP fluorophore on ChR2 could also be removed completely to reduce possible effects of ECFP mediated fluorophore assisted light inactivation, and transfected cells could still be identified by their mRFP1 fluorescence.

\section{BDNF cell-to-cell transfer}

The activity-dependent expression, localization and release of BDNF is diverse and complex. The mature BDNF amino acid sequence, consisting of 119 residues, is highly conserved in all mammals, and mRNA for BDNF and protein can be found throughout the rat brain (Conner et al., 1997; Ernfors et al., 1990) and similar distributions have been found in primates. Multiple promotors control the expression of 11 different BDNF mRNA splice variants (Aid et al., 2007). Interestingly, with the exception of one splice variant, were 8 amino acids can be added to the $\mathrm{N}$-terminal part of the protein, all mRNAs code for the very same protein. There are also amino acid differences between species that should be respected, e.g. V23A and G35A propeptide residues from mouse to rat. The exchange from valine and glycine to alanine does not change polarity or size significantly and therefore, these differences are unlikely to affect protein function, but a single amino acid difference in the propeptide of BDNF can lead to severe symptoms, as it has been described for the V66M polymorphism (Chen et al., 2004). To exclude for any such problems in differences in BDNF function between species, we focused only on the overexpression of the rat BDNF in cultures derived from rat tissue.

The presence of so many different BDNF mRNAs suggests a differential sorting of the various mRNAs and local expression. Indeed BDNF mRNA is differentially sorted to axons or dendrites in an activity dependent manner, implying a function of endogenous BDNF through local activity specific translation and release (Baj et al., 2011; Tongiorgi et al., 1997). However, while there is a mutual agreement about the presence and sorting of BDNF mRNA, the subcellular localization of the protein and its function is a constant source of controversy (Edelmann et al., 2014). It has been reported that overexpressed BDNF, that is produced in the cell body, is then transported to and released from both pre- and postsynaptic sites of neurons in an activity dependent fashion (Kohara et al., 2001; Kuczewski et al., 2008), while other studies report exclusive presynaptic localization (Dieni et al., 2012). And while BDNF mRNA is supposedly absent in astrocytes (Ernfors et al., 1990), released BDNF can be taken up by astrocytes influence their morphology (Ohira et al., 2007; Ohira et al., 2005) and possibly even be re-released (Bergami et al., 2008), suggesting a bidirectional signaling mechanism between neurons and astrocytes. Besides all these interesting implications, the release of BDNF by neurons and subsequent uptake by other cells has to our knowledge not been conclusively shown in any of these studies. They mostly report release onto other cells, but not transfer (Kohara et al., 2001) or uptake of recombinant BDNF that has been added to the medium without the previous release (Bergami et al., 2008). These studies strongly suggests such a connection between release 
and uptake, but there are nevertheless many more open questions, including whether neuronal activity affects transfer, whether release and possible uptake occur pre- or post-synaptically or both, and if there is bidirectional transfer and signaling between astrocytes and neurons or other cell types.

During the course of this project, we surprisingly observed a strong and prominent spread of the BDNF-mRFP1 signal in culture, labeling many more cells than can be transfected using calcium phosphate. To investigate the possibility of cell-to-cell transfer and to identify BDNF-mRFP1 producing cells, we combined the BDNF-mRFP1 sequence with a P2A peptide sequence linked to either EGFP or ECFP and expressed it under the control of the CamKIl $\alpha$ promotor, specific for excitatory neurons. The P2A peptide sequence allows for the coding of multiple peptides using a single ORF transcription unit, found in the Picornaviridae family (Ryan et al., 1991), where the 2A found in porcine teschovirus-1 (P2A) was most potent in producing separate polypeptides (Kim et al., 2011). The use of a P2A expression cassette also excludes for the possibility that the mRNA for BDNFmRFP1 was transferred from neurons to astrocytes via exosomes or extracellular vesicles, potentially leading to local translation (Ridder et al., 2014). Although we were expressing our BDNF-mRFP1-P2A-EGFP construct under control of the CaMKIla promotor, we still observed a remarkable spread of mRFP1 signal in neighboring cells around the EGFP positive cells. We were quite surprised that in decades of BDNF research using fluorescently labeled BDNF overexpression, similar observations have not been published, and set out to investigate the reason for this and in parallel, to identify the cells taking up the overexpressed and released BDNF-mRFP1.

When we performed standard ICC and compared the observed BDNF-mRFP1 intensities in different culture and treatment conditions, we found that treatment with the antimitotic agent AraC from DIV7 onward, as well as fixation and permeabilization of the culture resulted in a dramatic reduction of BDNF-mRFP1 fluorescence. Some mRFP1 signal remains and can still be detected, however the largest proportion is gone. Interestingly, in initial experiments in which we transfected BDNF-EGFP, the most commonly used BDNF-tagged fluorophore, we did not observe transfer to neighboring cells. By using $25 \mathrm{mM} \mathrm{NH}_{4} \mathrm{Cl}$, however, we could show that the previously seen absence of BDNF-EGFP signal is most likely because of acidic quenching in the subcellular compartments (Santi et al., 2006); addition of $25 \mathrm{mM} \mathrm{NH} 4 \mathrm{Cl}$ to de-acidify internal compartments revealed an equivalent amount of transfer of BDNF-EGFP to neighboring cells, compared to BDNF-mRFP1. These two observations, the quenching of EGFP in target cells and the loss of mRFP1 fluorescence during ICC in addition to the common application of AraC to cultures, could explain at least in part why such cell-to-cell transfer behavior of BDNF has not been reported before. Another possibility could be our use of the CaMKII $\alpha$ promotor, which may lead to higher expression levels than previous studies.

We could show that indeed BDNF-mRFP1 fluorescence co-occurs with other neurons identified by Map2 immunoreactivity or synapsin promotor activity. We also found some BDNF-mRFP1 in NG2 immunoreactive cells, presumably polydendrocytes (Nishiyama et al., 2009), but the most substantial BDNF-mRFP1 signal co-occurred with the somata of GFAP immunoreactive 
cells, i.e. astrocytes. We also tested Iba-1, for detection of microglia cells, but did not find Iba-1 immunoreactive cells in our cultures. It is unlikely that no microglia cells are present in these cultures and more likely that there was a problem with the ICC. To test this, different antibodies could be tested.

In order to confirm the actual uptake of BDNF, we performed live-labeling of the GFP and RFP fluorophore with preconjugated primary and secondary antibodies under non-permeabilizing conditions. Under these conditions antibodies detected the control epitope of Nrx, but failed to detect the large BDNF-mRFP1 accumulations in the somata. We can therefore conclude that BDNF-mRFP1 is internalized. The large quantities of the transferred BDNF-mRFP1 suggest some sort of regulated uptake using target receptors.

Attaching 224 amino acid peptides (EGFP or mRFP1) via an 11 amino acid long linker to a 249 amino acid ProBDNF might obscure the biological functions of BDNF. Fluorescently tagged BDNF has been reported to be physiologically active (Cubitt et al., 1995), and to behave in a manner indistinguishable from untagged BDNF (Haubensak et al., 1998; Kojima et al., 2001). Furthermore the endocytosis of EYFP-tagged recombinant BDNF is reported not to differ from that of native BDNF (Santi et al., 2006). In our experiments, untagged BDNF expressed in neurons was also transferred to astrocytes, in a manner similar to fluorescently tagged BDNF, thus confirming that the fluorescent tag does not severely affect physiological functions of BDNF.

In vitro cultures are often criticized for their differences to physiological in vivo conditions. To verify cell transfer under physiological conditions and address the question of release and transfer directionality from neurons, we injected crude CaMKIl $\alpha$-BDNF-mRFP1-P2A-ECFP AAV1/2 extracts, produced in HEK293 cells, into the CA3 or CA1 region of organotypic hippocampal brain slices. With this approach, we hoped to determine transfer directionality from CA3 to CA1 (i.e. pre to postsynaptic) or the other way around. To our surprise in this system the ECFP fluorophore was absent. We therefore swapped the ECFP to EGFP, hoping that this will improve the strength of the signal and used constructs packed into professionally purified AAV6 particles, which can presumably handle larger inserts, but again found an absence of the EGFP fluorophore, even though both constructs work well for calcium phosphate transfection of dissociated cultures. A possible explanation for this could be that during the packaging process of constructs that are too large, the single stranded insert is cropped (Dong et al., 2010). When the insert is cropped on the $3^{\prime}$ end it loses the downstream fluorophore and when the insert is cropped on the $5^{\prime}$ end this could affect the promotor and simply reduce the observed transduction efficiency. Including the two ITRs, our insert is slightly above $5 \mathrm{~kb}$. It has been reported that the limit for most AAVs is around $4.7 \mathrm{~kb}$ (Dong et al., 2010). Inserts larger than that will suffer from dramatically decreased packaging efficiency. Because the mRFP1-P2A-EGFP construct was successfully transduced and expressed with high efficiency, we can exclude the P2A sequence and reduce the problem to an issue of insert size, cropping and decreased packaging efficiency. Nevertheless, we also observed a local spread of the mRFP1 fluorescence around the site 
of injection, including cells outside of the pyramidal cell layer. This demonstrates local release of BDNF-mRFP1 and uptake by non-CaMKIl $\alpha$ promotor active cells in a physiological context.

To quantify the differential uptake by neurons and astrocytes without having to perform ICC, potentially decreasing mRFP1 fluorescence, we developed a four fluorophore-based live-labeling approach, in which dissociated neuronal cultures are transfected with CaMKIl $\alpha$ promotor controlled BDNF-mRFP1-P2A-ECFP. Subsequently the culture is transduced with AAV1/2 virions, labeling neurons and astrocytes by synapsin or GFAP2.2 specific expression of the LSSmOrange or EGFP fluorophores, respectively. These fluorophores could easily be detected and separated using conventional confocal microscopy and image analysis. Using ICC for the detection of Map2 and GFAP immunoreactive cells, we could confirm that AAV1/2 transduction successfully labels $80-90 \%$ of astrocytes and neurons, comparable to what has been reported in the literature (Royo et al., 2008). With this approach, we could show astrocytes and not neurons, are the primary recipients of neuronally overexpressed and released BDNF. Because we use overexpression of a protein for which translation is otherwise tightly regulated, our results have to be carefully interpreted, but astrocytes may play an important role in the regulation of BDNF signaling, perhaps by keeping the extracellular concentrations low and locally confined. However, BDNF is not without physiological function for astrocytes. Astrocytes express the p75 receptor and a truncated version of the TrkB receptor, missing the intracellular tyrosine kinase domain (Aroeira et al., 2015; Bergami et al., 2008; Rose et al., 2003). Previous work by others showed that exposure to brief BDNF application led to a rise of calcium concentrations in astrocytes (Rose et al., 2003). It can therefore be assumed, that the uptake of BDNF is not purely buffering, but also has physiological consequences for astrocytes. To study the fate of taken up BDNF-mRFP1 in neurons and astrocytes, we used electroporation to transfect dissociated neuronal cultures before plating with fluorescently labeled marker proteins for diverse subcellular compartments involved in protein degradation, sorting and processing, including the endosomal markers EEA1, Rab5 and Rab7, the lysosomal marker Lamp1 or the autophagosomal marker LC3, and subsequently transfected them with our BDNF construct using calcium phosphate. Because overexpression of proteins will eventually cause accumulations of the respective proteins in lysosomes, constructs were validated by ICC. There were no significant differences found in the localizations of the ICC staining and the fluorescent marker protein. Furthermore, if the marker proteins would eventually end up in the same compartments, we would expect similar colocalization results for all different conditions, which was not the case. Endocytosed BDNF-mRFP1 did not colocalize with the early endosomal markers EEA1 and Rab5 (Huotari and Helenius, 2011; Wilson et al., 2000) or the autophagosomal marker LC3 (Cherra et al., 2010), but exhibited significant colocalization with the late endosomal and lysosomal marker proteins Rab7 and Lamp1 (Humphries et al., 2011; Huotari and Helenius, 2011; Vanlandingham and Ceresa, 2009).

Primary endocytic vesicles fuse with and deliver their extracellular cargo to early endosomes at the peripheral cytoplasm, where they reside for approximately 5-18 min, while the early endosome accumulates further cargo. Subsequently the early endosome is transported along 
microtubules towards the perinuclear space. During this time it starts accumulating hydrolases and forms intraluminal vesicles, which mature during its transition into a late endosome. The switch from Rab5 to Rab7 occurs during maturation. Mature late endosomes are typically round or oval shaped and have a diameter of $250-1000 \mathrm{~nm}$. When they arrive at the perinuclear area they fuse with other late endosomes to form larger bodies and eventually fuse with lysosomes, where most of the components of late endosomes will be degraded (Huotari and Helenius, 2011). Autophagosomes engulf cytoplasmic components, including cytosolic proteins and organelles and fuse with lysosomes to form autolysosomes. Our data suggest that the largest proportion of endocytosed BDNF-mRFP1 goes down this path and finally ends up in lysosomes, where it is degraded. There were no significant differences found between the colocalization of taken up BDNF-mRFP1 between neurons and astrocytes with specific compartments, suggesting that in both cell types uptake may occur partly through similar mechanisms. Rab7 and Lamp1 were expected to colocalize significantly (Humphries et al., 2011), something that we can confirm by their similar colocalization with BDNF-mRFP1. The strong BDNF-mRFP1 colocalization with marker proteins that represent the late endosomal to lysosomal stages, suggests protein degradation instead of re-release. However, we cannot exclude that uptake and subsequent re-release occurs as previously reported (Bergami et al., 2008). The experiments in which re-release has been observed comes from the application of recombinant ProBDNF directly to the culture, under the assumption that BDNF is primarily released by neurons as ProBDNF, but the release of ProBDNF is controversial. Other than the TrkB receptor variants (fulllength and the two known truncated isoforms) the p75 receptor, which binds ProBDNF, is reported to be expressed only weakly in the adult cortex or hippocampus and dissociated neuron and astrocytes (Rudge et al., 1994; Yan and Johnson, 1988, 1989; Yan et al., 1997). Further experiments with the application of recombinant BDNF support the uptake by neurons mostly via the TrkB receptor (Santi et al., 2006). Indeed there is sufficient evidence to assume that BDNF is mostly processed intracellular and released in its mature form (Dieni et al., 2012; Matsumoto et al., 2008).

\section{Release of ProBDNF versus mature BDNF and physiological consequences for astrocytes}

We confirmed the functional relevance of the overexpressed BDNF-mRFP1 by demonstrating its ability to bind to the TrkB receptor. Because there was so much more BDNF-mRFP1 found in astrocytes than in neurons, we wondered if the released form is mostly ProBDNF binding to the p75 receptor on astrocytes or if neurons simply lack sufficient TrkB receptor levels to compete with astrocytes for available BDNF. We therefore overexpressed TrkB in neurons, which resulted in a dramatic increase of the BDNF-mRFP1 found in neurons, from $26 \%$ to $74 \%$ of the total BDNF-mRFP1, while at the same time BDNF-mRFP1 in astrocytes reduced from $58 \%$ to $11 \%$. This experiment demonstrated that first, BDNF-mRFP1 can bind to its native receptor and second, that the most abundant form of BDNF released by neurons is the mature form, although we cannot completely rule out extracellular processing. 
During these experiments, we also noticed that the shift in uptake by neuronal expression of TrkB was accompanied by a decline of astrocytic abundance in the culture of about $50 \%$. The decline in the astrocytic abundance is highly interesting. Small fluctuations in cell abundance can naturally occur between cultures, but they are not commonly so dramatic. This poses the question whether the BDNF-mRFP1 overexpression had physiological consequences on the astrocytic population. We therefore tested the effect of increasing the amount of released BDNF-mRFP1 by increasing neuronal activity in the culture using $10 \mathrm{mM}$ extracellular $\mathrm{KCl}$ or a protocol for the induction of chemical LTP. While $\mathrm{KCl}$ stimulation only increased the released BDNF-mRFP1 slightly and had no obvious influence on differential uptake or abundance of individual cell types, the cLTP protocol led to a marked increase in released BDNF-mRFP1 from which only the astrocytic population appeared to benefit. While the neuronal population remained stable and took up the same amount of BDNF-mRFP1 as seen under control condition, there was relatively less BDNF-mRFP1 in astrocytes. In addition, we found that astrocyte abundance was significantly increased, hence either cLTP or excess in BDNF-mRFP1 appears to stimulate astrocyte proliferation. But the excess astrocytes had decreased amounts of BDNF-mRFP1 taken up by individual cells. In separate experiments where we tested for changes in GFAP immunoreactivity, $10 \mathrm{mM} \mathrm{KCl}$ or CLTP alone did not significantly increase the number of astrocytes. Only BDNF-mRFP1 overexpression alone and in combination with the cLTP stimulation did. We also tested the effects of application of a TrkB Immunoglobulin G, which scavenges free BDNF, overexpression of TrkB, and application of recombinant BDNF. None of these treatments affected GFAP immunoreactivity. It is expected that there is only little endogenous BDNF available (Dieni et al., 2012); therefore it is not surprising, that the TrkB Immunoglobulin G application and the TrkB overexpression under normal conditions did not show any effect. However, the absence of an effect with TrkB overexpression also excludes the calcium phosphate transfection as a stimulating source of astrocytic proliferation. We also tried the application of the TrkB Immunoglobulin G on BDNF-mRFP1 overexpressing cultures, where it led to a decrease of released BDNF-mRFP1 by about $60 \%$, consequently decreasing the signal found in all three groups (neurons, astrocytes and unassigned cells), but it did not appear to be significantly low enough to have an effect on any cell type abundance. For the added recombinant BDNF, it is possible that we did not see effects because the concentration was not sufficient or a local high concentration source of released BDNF at neuron-astrocyte interfaces is necessary to see effects on astrocyte abundance. We tested both BDNF produced in bacterial strains and in eukaryotic cells, because it has been reported that the absence of essential post-translational modifications renders the BDNF produced in bacteria biologically inactive. We expect that BDNF concentrations achieved through overexpression may be greater than the ones we could achieve with bath application. It might be worth performing the application again and subsequently test GFAP and BDNF immunoreactivity to compare BDNF levels. 


\section{Conclusion and future directions}

During this dissertation, we investigated a novel approach towards the control over synaptic to subsynaptic neuronal activity by using optogenetics in combination with an illumination approach originally inspired by STED/RESOLFT microscopy. We proposed two different approaches: 1) to use switchable channel variants, which can be activated by one wavelength and inactivated by another wavelength in an area of coillumination and 2) to use depolarizing channels and hyperpolarizing ionpumps in combination, to eliminate depolarizing voltage changes by counterbalancing currents in the coilluminated area, therefore confining the region of activation. We identified suitable channel candidates and features that aid to reduce photocurrents under coillumination. Furthermore, we demonstrated the feasibility of both approaches for the stimulation of highly focal membrane areas. But we also demonstrate that at present this application is not realistic, due to the weak conductances of the available channels. The knowledge from this work will be useful for future applications that make use of the investigated channel properties and different illumination approaches. Nevertheless, the field of optogenetics is quickly progressing and it is very likely that stronger conducting variants will be found in time. It could, for example, be interesting to focus on photoswitchable ionotropic glutamate receptors (Berlin et al., 2016; Volgraf et al., 2006). These channels promise to reach much higher conductances, but remain to be tested in terms of whether they can easily be inactivated by coillumination for possible use to stimulate sub-diffraction limited membrane areas.

In addition, we tested the recruitment of BDNF to optogenetically stimulated areas and found an interesting effect, the recruitment of BDNF vesicles to stimulated sites, that cannot easily be explained by phototoxicity. Further experiments without a second fluorophore on the light-gated channel and using less intense light stimulation protocols should be considered in the future to rule out effects of light stimulation alone, in the absence of channelrhodopsins. Moreover, we demonstrated the transfer of neuronally expressed fluorescently labeled BDNF to nearby cells, something that has so far only been inferred, but not demonstrated and showed that in fact most BDNF is being taken up by astrocytes. Consequently, we provide further evidence for the physiological relevance of studies using fluorescently labeled BDNF by showing that the released BDNF binds to its target receptor, TrkB. We could show that the overexpression of TrkB in neurons altered the differential uptake of BDNF by neurons and astrocytes and had functional consequences on astrocyte proliferation. Follow-up experiments, in which either the p75NTR or the truncated TrkB variants are removed from astrocytes might provide further information about their contribution to the uptake by astrocytes and the presence of Pro- or mature BDNF. A possible approach for removing p75NTR or the truncated TrkB variants is to use the CRISPR/Cas9 system, a technique that has already been shown to work efficiently in postmitotic neurons (Incontro et al., 2014; Straub et al., 2014). Based on the diverse and complex regulation of BDNF transcripts, one could consider introducing CDNA that leads to the generation of the specific transcripts, to investigate their possible 
contribution to differentially sorted and controlled BDNF populations. We also used organotypic hippocampal slices to address the question of pre- or postsynaptic release and transfer directionality using AAV-mediated BDNF-mRFP1 overexpression. However, most likely because of packaging limits of the AAVs, we were not able to detect the cytosolic fluorophore in cells of origin. Approaches like dual AAV application, in which one large plasmid can be split and separately delivered by two AAVs (Duan et al., 2001; Ghosh et al., 2008), expanding the capacity of AAV vectors could help to address this question. 


\section{Acknowledgments}

I want to use this opportunity to thank the people that significantly contributed to my experience of studying Neuroscience in Göttingen during the last 4 years and directly or indirectly contributed to the development of this thesis.

Thank you Camin, I guess I have to thank you the most of all for accepting me as a student in your lab. The last few years have been an extraordinary experience to me, though full of challenging experiments and obstacles, you never stopped to believe in me and were always positive and supportive. You treat all the lab members with respect and one can only reverence you for the way you manage all these diverse and difficult projects. I am most grateful that you accepted me as student in your lab. I learned so much and to master so many different techniques. Your support let me travel to many important meetings, where I had the chance to talk to highly skilled and experienced scientist. These experiences were invaluable. Thank you.

I also want to take this chance and thank Stefan Hell and Detlev Schild for being my scientific advisors and providing critical input to the ideas that have shaped this dissertation. More than once I was pushed to think in directions I had not anticipated before.

Thank you, Michael, Sandra und Mirja, you are the core and heart of the program that made me come to Göttingen in the first place. Without your endless initiative, support and sometimes also patience, this program would not even be half as successful. You are doing a great job and I hope many more students will have the chance to experience that.

Sebastian Kügler vitally contributed to the AAV and BDNF part of my thesis, by providing us with constructs, potent viral particles and most notably valuable advice. Thank you for your invaluable support.

To my PhD colleagues in crime. I had a great time with all of you (for most of the time (:)). We helped each other when help was needed and formed kind of a second family where everyone was unique and irreplaceable. Vinita, thank you deeply for your light-hearted spirit that always made everyone smile (although I think you should stop singing ABBA songs in the lab). Ankit and Charlie, it was a great pleasure to work alongside of two so skilled and motivated students and I very much appreciate our scientific debates and our rebellious early morning journal clubs.

I had a great time with all of you and your spirit and help is part of this thesis!

I also want to thank our former lab members Yo Shinoda and Jan Schrader. You guys were an essential part of my experience in this lab and you helped me out with my projects when I was new and inexperienced. Thank you Yo for your scientific input and lessons about microscopy and electrophysiology and thank you Jan for your pragmatic and critical input about life and also some science. Thank you Binu for sharing your experience in working with organotypic cultures with me and thank you Katja for your valuable input and help with constructs.

Plinio, you are not part of our lab, but that actually makes no difference to me. You were always part of my second lab family. Thank you for the interesting conversations during lunchtime and the 
initiative behind all biking and hiking tours we have done throughout the last years, no matter how the weather was. There were some truly memorable trips and this was contributing a lot to my sanity.

Peter Wehner and Nils Halbsgut helped me out a lot when I was encountering problems with one of the microscopes, Frank Kötting made huge contributions by designing and repairing many of the tools that were vital to conduct my experiments and Matthias Weyl made sure that everything was working fine with the IT behind this work. Thank you guys for your support.

Of course, I have to deeply thank Sandra Ott-Gebauer. Sandra, you are officially not even belonging to our lab, but I cannot even imagine where we would be without all your invaluable advice and shared experience.

And last but by no means the least I want to deeply thank the administration and coordination management of the European Neuroscience Institute, and especially Christiane Becker, for her perpetual assistance in the jungle of bureaucracy.

This place is too small to name the endless names of people that supported me in Göttingen, but in fact there were many more. I want to extend my gratitude to all those who I met and became good friends with during the last 4 years.

Thank you. 


\section{Declaration of independent work}

Herewith I declare, that I prepared the presented doctoral thesis entitled "Nanoscale Probing of Single Synapse Function and BDNF Cell-to-Cell Transfer" on my own and with no other sources and aids than quoted.

Göttingen, $31^{\text {st }}$ March 2016

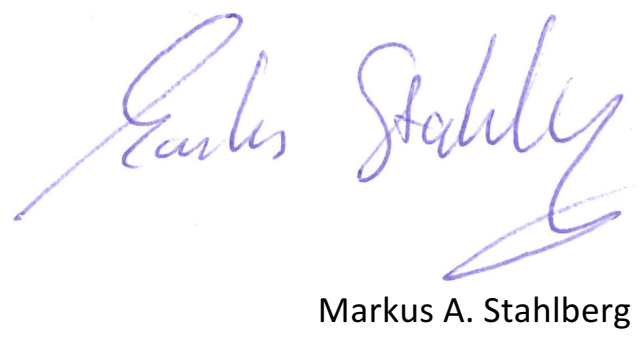




\section{References}

Adams, S.R., and Tsien, R.Y. (1993). Controlling cell chemistry with caged compounds. Annual review of physiology $55,755-784$.

Aid, T., Kazantseva, A., Piirsoo, M., Palm, K., and Timmusk, T. (2007). Mouse and rat BDNF gene structure and expression revisited. Journal of neuroscience research 85, 525-535.

Akerboom, J., Carreras Calderon, N., Tian, L., Wabnig, S., Prigge, M., Tolo, J., Gordus, A., Orger, M.B., Severi, K.E., Macklin, J.J., et al. (2013). Genetically encoded calcium indicators for multi-color neural activity imaging and combination with optogenetics. Frontiers in molecular neuroscience 6, 2.

Akerboom, J., Chen, T.W., Wardill, T.J., Tian, L., Marvin, J.S., Mutlu, S., Calderon, N.C., Esposti, F., Borghuis, B.G., Sun, X.R., et al. (2012). Optimization of a GCaMP calcium indicator for neural activity imaging. The Journal of neuroscience : the official journal of the Society for Neuroscience 32, 1381913840.

Allocca, M., Doria, M., Petrillo, M., Colella, P., Garcia-Hoyos, M., Gibbs, D., Kim, S.R., Maguire, A., Rex, T.S., Di Vicino, U., et al. (2008). Serotype-dependent packaging of large genes in adeno-associated viral vectors results in effective gene delivery in mice. The Journal of clinical investigation 118, 19551964.

An, J.J., Gharami, K., Liao, G.Y., Woo, N.H., Lau, A.G., Vanevski, F., Torre, E.R., Jones, K.R., Feng, Y., Lu, B., et al. (2008). Distinct role of long 3' UTR BDNF mRNA in spine morphology and synaptic plasticity in hippocampal neurons. Cell 134, 175-187.

Andrasfalvy, B.K., and Magee, J.C. (2001). Distance-dependent increase in AMPA receptor number in the dendrites of adult hippocampal CA1 pyramidal neurons. The Journal of neuroscience : the official journal of the Society for Neuroscience 21, 9151-9159.

Aroeira, R.I., Sebastiao, A.M., and Valente, C.A. (2015). BDNF, via truncated TrkB receptor, modulates GlyT1 and GlyT2 in astrocytes. Glia 63, 2181-2197.

Baj, G., Leone, E., Chao, M.V., and Tongiorgi, E. (2011). Spatial segregation of BDNF transcripts enables BDNF to differentially shape distinct dendritic compartments. Proceedings of the National Academy of Sciences of the United States of America 108, 16813-16818.

Bamann, C., Gueta, R., Kleinlogel, S., Nagel, G., and Bamberg, E. (2010). Structural guidance of the photocycle of channelrhodopsin-2 by an interhelical hydrogen bond. Biochemistry 49, 267-278.

Bamann, C., Kirsch, T., Nagel, G., and Bamberg, E. (2008). Spectral characteristics of the photocycle of channelrhodopsin-2 and its implication for channel function. Journal of molecular biology $375,686-$ 694.

Banghart, M., Borges, K., Isacoff, E., Trauner, D., and Kramer, R.H. (2004). Light-activated ion channels for remote control of neuronal firing. Nature neuroscience 7, 1381-1386.

Banker, G.A., and Cowan, W.M. (1977). Rat hippocampal neurons in dispersed cell culture. Brain research 126, 397-342.

Barde, Y.A. (1988). What, if anything, is a neurotrophic factor? Trends in neurosciences 11, 343-346. 
Barde, Y.A., Edgar, D., and Thoenen, H. (1982). Purification of a new neurotrophic factor from mammalian brain. The EMBO journal 1, 549-553.

Bartels, E., Wassermann, N.H., and Erlanger, B.F. (1971). Photochromic activators of the acetylcholine receptor. Proceedings of the National Academy of Sciences of the United States of America 68, 1820-1823.

Baxter, G.T., Radeke, M.J., Kuo, R.C., Makrides, V., Hinkle, B., Hoang, R., Medina-Selby, A., Coit, D., Valenzuela, P., and Feinstein, S.C. (1997). Signal transduction mediated by the truncated trkB receptor isoforms, trkB.T1 and trkB.T2. The Journal of neuroscience : the official journal of the Society for Neuroscience 17, 2683-2690.

Bekkers, J.M., and Stevens, C.F. (1989). NMDA and non-NMDA receptors are co-localized at individual excitatory synapses in cultured rat hippocampus. Nature 341, 230-233.

Benson, D.L., Watkins, F.H., Steward, O., and Banker, G. (1994). Characterization of GABAergic neurons in hippocampal cell cultures. Journal of neurocytology 23, 279-295.

Bergami, M., Santi, S., Formaggio, E., Cagnoli, C., Verderio, C., Blum, R., Berninger, B., Matteoli, M., and Canossa, M. (2008). Uptake and recycling of pro-BDNF for transmitter-induced secretion by cortical astrocytes. The Journal of cell biology 183, 213-221.

Berlin, S., Szobota, S., Reiner, A., Carroll, E.C., Kienzler, M.A., Guyon, A., Xiao, T., Tauner, D., and Isacoff, E.Y. (2016). A family of photoswitchable NMDA receptors. eLife 5.

Berndt, A., Lee, S.Y., Ramakrishnan, C., and Deisseroth, K. (2014). Structure-guided transformation of channelrhodopsin into a light-activated chloride channel. Science 344, 420-424.

Berndt, A., Schoenenberger, P., Mattis, J., Tye, K.M., Deisseroth, K., Hegemann, P., and Oertner, T.G. (2011). High-efficiency channelrhodopsins for fast neuronal stimulation at low light levels. Proceedings of the National Academy of Sciences of the United States of America 108, 7595-7600.

Berndt, A., Yizhar, O., Gunaydin, L.A., Hegemann, P., and Deisseroth, K. (2009). Bi-stable neural state switches. Nature neuroscience 12, 229-234.

Berninger, B., and Poo, M. (1996). Fast actions of neurotrophic factors. Current opinion in neurobiology 6, 324-330.

Berninger, B., Schinder, A.F., and Poo, M.M. (1999). Synaptic reliability correlates with reduced susceptibility to synaptic potentiation by brain-derived neurotrophic factor. Learn Mem 6, 232-242.

Bibel, M., and Barde, Y.A. (2000). Neurotrophins: key regulators of cell fate and cell shape in the vertebrate nervous system. Genes \& development 14, 2919-2937.

Biffo, S., Offenhauser, N., Carter, B.D., and Barde, Y.A. (1995). Selective binding and internalisation by truncated receptors restrict the availability of BDNF during development. Development 121, 24612470.

Binder, D.K., and Scharfman, H.E. (2004). Brain-derived neurotrophic factor. Growth Factors 22, 123131.

Birnboim, H.C., and Doly, J. (1979). A rapid alkaline extraction procedure for screening recombinant plasmid DNA. Nucleic acids research 7, 1513-1523. 
Bliss, T.V., and Collingridge, G.L. (1993). A synaptic model of memory: long-term potentiation in the hippocampus. Nature 361, 31-39.

Borodina, T.A., Lehrach, H., and Soldatov, A.V. (2003). DNA purification on homemade silica spincolumns. Analytical biochemistry 321, 135-137.

Boyden, E.S., Zhang, F., Bamberg, E., Nagel, G., and Deisseroth, K. (2005). Millisecond-timescale, genetically targeted optical control of neural activity. Nature neuroscience 8, 1263-1268.

Brewer, G.J., Torricelli, J.R., Evege, E.K., and Price, P.J. (1993). Optimized survival of hippocampal neurons in B27-supplemented Neurobasal, a new serum-free medium combination. Journal of neuroscience research 35, 567-576.

Chao, M.V., and Bothwell, M. (2002). Neurotrophins: to cleave or not to cleave. Neuron 33, 9-12.

Chen, C.A., and Okayama, H. (1988). Calcium phosphate-mediated gene transfer: a highly efficient transfection system for stably transforming cells with plasmid DNA. BioTechniques 6, 632-638.

Chen, T.W., Wardill, T.J., Sun, Y., Pulver, S.R., Renninger, S.L., Baohan, A., Schreiter, E.R., Kerr, R.A., Orger, M.B., Jayaraman, V., et al. (2013). Ultrasensitive fluorescent proteins for imaging neuronal activity. Nature 499, 295-300.

Chen, W.G., Chang, Q., Lin, Y., Meissner, A., West, A.E., Griffith, E.C., Jaenisch, R., and Greenberg, M.E. (2003a). Derepression of BDNF transcription involves calcium-dependent phosphorylation of MeCP2. Science 302, 885-889.

Chen, W.G., West, A.E., Tao, X., Corfas, G., Szentirmay, M.N., Sawadogo, M., Vinson, C., and Greenberg, M.E. (2003b). Upstream stimulatory factors are mediators of Ca2+-responsive transcription in neurons. The Journal of neuroscience : the official journal of the Society for Neuroscience 23, 2572-2581.

Chen, Z.Y., leraci, A., Teng, H., Dall, H., Meng, C.X., Herrera, D.G., Nykjaer, A., Hempstead, B.L., and Lee, F.S. (2005). Sortilin controls intracellular sorting of brain-derived neurotrophic factor to the regulated secretory pathway. The Journal of neuroscience : the official journal of the Society for Neuroscience 25, 6156-6166.

Chen, Z.Y., Jing, D., Bath, K.G., leraci, A., Khan, T., Siao, C.J., Herrera, D.G., Toth, M., Yang, C., McEwen, B.S., et al. (2006). Genetic variant BDNF (Val66Met) polymorphism alters anxiety-related behavior. Science 314, 140-143.

Chen, Z.Y., Patel, P.D., Sant, G., Meng, C.X., Teng, K.K., Hempstead, B.L., and Lee, F.S. (2004). Variant brain-derived neurotrophic factor (BDNF) (Met66) alters the intracellular trafficking and activitydependent secretion of wild-type BDNF in neurosecretory cells and cortical neurons. The Journal of neuroscience : the official journal of the Society for Neuroscience 24, 4401-4411.

Cherra, S.J., 3rd, Kulich, S.M., Uechi, G., Balasubramani, M., Mountzouris, J., Day, B.W., and Chu, C.T. (2010). Regulation of the autophagy protein LC3 by phosphorylation. The Journal of cell biology 190, 533-539.

Chow, B.Y., Han, X., Dobry, A.S., Qian, X., Chuong, A.S., Li, M., Henninger, M.A., Belfort, G.M., Lin, Y., Monahan, P.E., et al. (2010). High-performance genetically targetable optical neural silencing by light-driven proton pumps. Nature 463, 98-102. 
Chuong, A.S., Miri, M.L., Busskamp, V., Matthews, G.A., Acker, L.C., Sorensen, A.T., Young, A., Klapoetke, N.C., Henninger, M.A., Kodandaramaiah, S.B., et al. (2014). Noninvasive optical inhibition with a red-shifted microbial rhodopsin. Nature neuroscience 17, 1123-1129.

Clark, R.E., and Squire, L.R. (2013). Similarity in form and function of the hippocampus in rodents, monkeys, and humans. Proceedings of the National Academy of Sciences of the United States of America 110 Suppl 2, 10365-10370.

Climent, E., Sancho-Tello, M., Minana, R., Barettino, D., and Guerri, C. (2000). Astrocytes in culture express the full-length Trk-B receptor and respond to brain derived neurotrophic factor by changing intracellular calcium levels: effect of ethanol exposure in rats. Neuroscience letters 288, 53-56.

Cobb, S. (1965). Brain Size. Arch Neurol 12, 555-561.

Colombo, E., Cordiglieri, C., Melli, G., Newcombe, J., Krumbholz, M., Parada, L.F., Medico, E., Hohlfeld, R., Meinl, E., and Farina, C. (2012). Stimulation of the neurotrophin receptor TrkB on astrocytes drives nitric oxide production and neurodegeneration. The Journal of experimental medicine 209, 521-535.

Conner, J.M., Lauterborn, J.C., Yan, Q., Gall, C.M., and Varon, S. (1997). Distribution of brain-derived neurotrophic factor (BDNF) protein and mRNA in the normal adult rat CNS: evidence for anterograde axonal transport. The Journal of neuroscience : the official journal of the Society for Neuroscience 17, 2295-2313.

Connor, B., Young, D., Yan, Q., Faull, R.L., Synek, B., and Dragunow, M. (1997). Brain-derived neurotrophic factor is reduced in Alzheimer's disease. Brain research Molecular brain research 49, 71-81.

Coons, A.H., Creech, H.J., and Jones, R.N. (1941). Immunological properties of an antibody containing a fluorescence group. Proc Soc Exp Biol Med 47, 200-202.

Cubitt, A.B., Heim, R., Adams, S.R., Boyd, A.E., Gross, L.A., and Tsien, R.Y. (1995). Understanding, improving and using green fluorescent proteins. Trends in biochemical sciences 20, 448-455.

Dean, C., Liu, H., Staudt, T., Stahlberg, M.A., Vingill, S., Buckers, J., Kamin, D., Engelhardt, J., Jackson, M.B., Hell, S.W., et al. (2012). Distinct Subsets of Syt-IV/BDNF Vesicles Are Sorted to Axons versus Dendrites and Recruited to Synapses by Activity. The Journal of neuroscience : the official journal of the Society for Neuroscience 32, 5398-5413.

Deisseroth, K. (2011). Optogenetics. Nature methods 8, 26-29.

Dieni, S., Matsumoto, T., Dekkers, M., Rauskolb, S., lonescu, M.S., Deogracias, R., Gundelfinger, E.D., Kojima, M., Nestel, S., Frotscher, M., et al. (2012). BDNF and its pro-peptide are stored in presynaptic dense core vesicles in brain neurons. The Journal of cell biology 196, 775-788.

DiStefano, P.S., Friedman, B., Radziejewski, C., Alexander, C., Boland, P., Schick, C.M., Lindsay, R.M., and Wiegand, S.J. (1992). The neurotrophins BDNF, NT-3, and NGF display distinct patterns of retrograde axonal transport in peripheral and central neurons. Neuron 8, 983-993.

Dobrucki, J.W., Feret, D., and Noatynska, A. (2007). Scattering of exciting light by live cells in fluorescence confocal imaging: phototoxic effects and relevance for FRAP studies. Biophysical journal 93, 1778-1786. 
Dong, B., Nakai, H., and Xiao, W. (2010). Characterization of genome integrity for oversized recombinant AAV vector. Molecular therapy : the journal of the American Society of Gene Therapy 18, 87-92.

Du, J., Feng, L., Yang, F., and Lu, B. (2000). Activity- and $\mathrm{Ca}(2+)$-dependent modulation of surface expression of brain-derived neurotrophic factor receptors in hippocampal neurons. The Journal of cell biology 150, 1423-1434.

Duan, D., Yue, Y., and Engelhardt, J.F. (2001). Expanding AAV packaging capacity with trans-splicing or overlapping vectors: a quantitative comparison. Molecular therapy : the journal of the American Society of Gene Therapy 4, 383-391.

Edelmann, E., Cepeda-Prado, E., Franck, M., Lichtenecker, P., Brigadski, T., and Lessmann, V. (2015). Theta Burst Firing Recruits BDNF Release and Signaling in Postsynaptic CA1 Neurons in Spike-TimingDependent LTP. Neuron 86, 1041-1054.

Edelmann, E., Lessmann, V., and Brigadski, T. (2014). Pre- and postsynaptic twists in BDNF secretion and action in synaptic plasticity. Neuropharmacology 76 Pt C, 610-627.

Egan, M.F., Kojima, M., Callicott, J.H., Goldberg, T.E., Kolachana, B.S., Bertolino, A., Zaitsev, E., Gold, B., Goldman, D., Dean, M., et al. (2003). The BDNF val66met polymorphism affects activitydependent secretion of BDNF and human memory and hippocampal function. Cell 112, 257-269.

Elliott, R.C., Inturrisi, C.E., Black, I.B., and Dreyfus, C.F. (1994). An improved method detects differential NGF and BDNF gene expression in response to depolarization in cultured hippocampal neurons. Brain research Molecular brain research 26, 81-88.

Erbguth, K., Prigge, M., Schneider, F., Hegemann, P., and Gottschalk, A. (2012). Bimodal activation of different neuron classes with the spectrally red-shifted channelrhodopsin chimera C1V1 in Caenorhabditis elegans. PloS one 7, e46827.

Ernfors, P., Kucera, J., Lee, K.F., Loring, J., and Jaenisch, R. (1995). Studies on the physiological role of brain-derived neurotrophic factor and neurotrophin-3 in knockout mice. The International journal of developmental biology 39, 799-807.

Ernfors, P., Lee, K.F., and Jaenisch, R. (1994). Mice lacking brain-derived neurotrophic factor develop with sensory deficits. Nature $368,147-150$.

Ernfors, P., Wetmore, C., Olson, L., and Persson, H. (1990). Identification of cells in rat brain and peripheral tissues expressing mRNA for members of the nerve growth factor family. Neuron 5, 511526.

Farhadi, H.F., Mowla, S.J., Petrecca, K., Morris, S.J., Seidah, N.G., and Murphy, R.A. (2000). Neurotrophin-3 sorts to the constitutive secretory pathway of hippocampal neurons and is diverted to the regulated secretory pathway by coexpression with brain-derived neurotrophic factor. The Journal of neuroscience : the official journal of the Society for Neuroscience 20,4059-4068.

Farina, M., van de Bospoort, R., He, E., Persoon, C.M., van Weering, J.R., Broeke, J.H., Verhage, M., and Toonen, R.F. (2015). CAPS-1 promotes fusion competence of stationary dense-core vesicles in presynaptic terminals of mammalian neurons. elife 4.

Feldbauer, K., Zimmermann, D., Pintschovius, V., Spitz, J., Bamann, C., and Bamberg, E. (2009). Channelrhodopsin-2 is a leaky proton pump. Proceedings of the National Academy of Sciences of the United States of America 106, 12317-12322. 
Figurov, A., Pozzo-Miller, L.D., Olafsson, P., Wang, T., and Lu, B. (1996). Regulation of synaptic responses to high-frequency stimulation and LTP by neurotrophins in the hippocampus. Nature 381, 706-709.

Fork, R.L. (1971). Laser stimulation of nerve cells in Aplysia. Science 171, 907-908.

Frerking, M., Malenka, R.C., and Nicoll, R.A. (1998). Brain-derived neurotrophic factor (BDNF) modulates inhibitory, but not excitatory, transmission in the CA1 region of the hippocampus. Journal of neurophysiology $80,3383-3386$.

Fryer, R.H., Kaplan, D.R., Feinstein, S.C., Radeke, M.J., Grayson, D.R., and Kromer, L.F. (1996). Developmental and mature expression of full-length and truncated TrkB receptors in the rat forebrain. The Journal of comparative neurology 374, 21-40.

Gee, K.R., Brown, K.A., Chen, W.N., Bishop-Stewart, J., Gray, D., and Johnson, I. (2000). Chemical and physiological characterization of fluo-4 $\mathrm{Ca}(2+)$-indicator dyes. Cell calcium 27, 97-106.

Ghosh, A., Yue, Y., Lai, Y., and Duan, D. (2008). A hybrid vector system expands adeno-associated viral vector packaging capacity in a transgene-independent manner. Molecular therapy : the journal of the American Society of Gene Therapy 16, 124-130.

Gibb, A.J., and Colquhoun, D. (1992). Activation of N-methyl-D-aspartate receptors by L-glutamate in cells dissociated from adult rat hippocampus. The Journal of physiology 456, 143-179.

Goetze, B., Grunewald, B., Baldassa, S., and Kiebler, M. (2004). Chemically controlled formation of a DNA/calcium phosphate coprecipitate: application for transfection of mature hippocampal neurons. Journal of neurobiology 60, 517-525.

Goodman, L.J., Valverde, J., Lim, F., Geschwind, M.D., Federoff, H.J., Geller, A.I., and Hefti, F. (1996). Regulated release and polarized localization of brain-derived neurotrophic factor in hippocampal neurons. Molecular and cellular neurosciences 7, 222-238.

Goslin, K., and Banker, G. (1991). Rat hippocampal neurons in low density culture. Culturing Nerve Cells / MIT Press, pp. 251-281.

Gottschalk, W., Pozzo-Miller, L.D., Figurov, A., and Lu, B. (1998). Presynaptic modulation of synaptic transmission and plasticity by brain-derived neurotrophic factor in the developing hippocampus. The Journal of neuroscience : the official journal of the Society for Neuroscience 18,6830-6839.

Gradinaru, V., Zhang, F., Ramakrishnan, C., Mattis, J., Prakash, R., Diester, I., Goshen, I., Thompson, K.R., and Deisseroth, K. (2010). Molecular and cellular approaches for diversifying and extending optogenetics. Cell 141, 154-165.

Graham, F.L., Smiley, J., Russell, W.C., and Nairn, R. (1977). Characteristics of a human cell line transformed by DNA from human adenovirus type 5 . The Journal of general virology 36, 59-74.

Greenberg, K.P., Pham, A., and Werblin, F.S. (2011). Differential targeting of optical neuromodulators to ganglion cell soma and dendrites allows dynamic control of center-surround antagonism. Neuron 69, 713-720.

Gunaydin, L.A., Yizhar, O., Berndt, A., Sohal, V.S., Deisseroth, K., and Hegemann, P. (2010). Ultrafast optogenetic control. Nature neuroscience 13, 387-392. 
Han, X., Chow, B.Y., Zhou, H., Klapoetke, N.C., Chuong, A., Rajimehr, R., Yang, A., Baratta, M.V., Winkle, J., Desimone, R., et al. (2011). A high-light sensitivity optical neural silencer: development and application to optogenetic control of non-human primate cortex. Front Syst Neurosci 5, 18.

Hartmann, D., Drummond, J., Handberg, E., Ewell, S., and Pozzo-Miller, L. (2012). Multiple approaches to investigate the transport and activity-dependent release of BDNF and their application in neurogenetic disorders. Neural plasticity 2012, 203734.

Hartmann, M., Heumann, R., and Lessmann, V. (2001). Synaptic secretion of BDNF after highfrequency stimulation of glutamatergic synapses. The EMBO journal 20, 5887-5897.

Haubensak, W., Narz, F., Heumann, R., and Lessmann, V. (1998). BDNF-GFP containing secretory granules are localized in the vicinity of synaptic junctions of cultured cortical neurons. Journal of cell science 111 ( Pt 11), 1483-1493.

Hauck, B., Chen, L., and Xiao, W. (2003). Generation and characterization of chimeric recombinant $A A V$ vectors. Molecular therapy : the journal of the American Society of Gene Therapy 7, 419-425.

Heaulme, M., Chambon, J.P., Leyris, R., Wermuth, C.G., and Biziere, K. (1987). Characterization of the binding of [3H]SR 95531, a GABAA antagonist, to rat brain membranes. Journal of neurochemistry $48,1677-1686$.

Hechst, B. (1932). Über einen Fall von Mikroencephalie ohne geistigen Defekt. Archiv für Psychiatrische Nervenkrankheiten 97, 64-76.

Hegemann, P. (2008). Algal sensory photoreceptors. Annual review of plant biology 59, 167-189.

Hegemann, P., and Moglich, A. (2011). Channelrhodopsin engineering and exploration of new optogenetic tools. Nature methods $8,39-42$.

Heymach, J.V., Jr., and Shooter, E.M. (1995). The biosynthesis of neurotrophin heterodimers by transfected mammalian cells. The Journal of biological chemistry $270,12297-12304$.

Hirase, H., Nikolenko, V., Goldberg, J.H., and Yuste, R. (2002). Multiphoton stimulation of neurons. Journal of neurobiology 51, 237-247.

Hirase, H., Nikolenko, V., and Yuste, R. (2012). Multiphoton stimulation of neurons and spines. Cold Spring Harbor protocols 2012, 472-475.

Hochbaum, D.R., Zhao, Y., Farhi, S.L., Klapoetke, N., Werley, C.A., Kapoor, V., Zou, P., Kralj, J.M., Maclaurin, D., Smedemark-Margulies, N., et al. (2014). All-optical electrophysiology in mammalian neurons using engineered microbial rhodopsins. Nature methods 11, 825-833.

Hofer, M.M., and Barde, Y.A. (1988). Brain-derived neurotrophic factor prevents neuronal death in vivo. Nature 331, 261-262.

Honoré, T., Davies, S.N., Drejer, J., Fletcher, E.J., Jacobsen, P., Lodge, D., and Nielsen, F.E. (1987). Potent and competitive antagonism at non-NMDA receptors by FG 9041 and FG 9065. Soc Neurosci Abstr 13, p. 383P.

Huang, E.J., and Reichardt, L.F. (2001). Neurotrophins: roles in neuronal development and function. Annual review of neuroscience $24,677-736$. 
Humphries, W.H.t., Szymanski, C.J., and Payne, C.K. (2011). Endo-lysosomal vesicles positive for Rab7 and LAMP1 are terminal vesicles for the transport of dextran. PloS one 6, e26626.

Huotari, J., and Helenius, A. (2011). Endosome maturation. The EMBO journal 30, 3481-3500.

Incontro, S., Asensio, C.S., Edwards, R.H., and Nicoll, R.A. (2014). Efficient, complete deletion of synaptic proteins using CRISPR. Neuron 83, 1051-1057.

Jahr, C.E., and Stevens, C.F. (1993). Calcium permeability of the N-methyl-D-aspartate receptor channel in hippocampal neurons in culture. Proceedings of the National Academy of Sciences of the United States of America 90, 11573-11577.

Jay, D.G. (1988). Selective destruction of protein function by chromophore-assisted laser inactivation. Proceedings of the National Academy of Sciences of the United States of America 85, 5454-5458.

Jiang, M., and Chen, G. (2006). High Ca2+-phosphate transfection efficiency in low-density neuronal cultures. Nature protocols 1, 695-700.

Jonas, P., and Sakmann, B. (1992). Glutamate receptor channels in isolated patches from CA1 and CA3 pyramidal cells of rat hippocampal slices. The Journal of physiology 455, 143-171.

Jovanovic, J.N., Czernik, A.J., Fienberg, A.A., Greengard, P., and Sihra, T.S. (2000). Synapsins as mediators of BDNF-enhanced neurotransmitter release. Nature neuroscience 3, 323-329.

Kaech, S., and Banker, G. (2006). Culturing hippocampal neurons. Nature protocols 1, 2406-2415.

Kandel, E.R. (2001). The molecular biology of memory storage: a dialogue between genes and synapses. Science 294, 1030-1038.

Kandel, E.R., Schwartz, J.H., and Jessell, T.M. (2000). Principles of Neural Science. McGraw-Hill Medical 4th edition.

Kantevari, S., Matsuzaki, M., Kanemoto, Y., Kasai, H., and Ellis-Davies, G.C. (2010). Two-color, twophoton uncaging of glutamate and GABA. Nature methods 7, 123-125.

Kaplan, D.R., Hempstead, B.L., Martin-Zanca, D., Chao, M.V., and Parada, L.F. (1991). The trk protooncogene product: a signal transducing receptor for nerve growth factor. Science 252, 554-558.

Kaplan, D.R., and Miller, F.D. (2000). Neurotrophin signal transduction in the nervous system. Current opinion in neurobiology 10, 381-391.

Kim, J.H., Lee, S.R., Li, L.H., Park, H.J., Park, J.H., Lee, K.Y., Kim, M.K., Shin, B.A., and Choi, S.Y. (2011). High cleavage efficiency of a $2 A$ peptide derived from porcine teschovirus- 1 in human cell lines, zebrafish and mice. PloS one 6, e18556.

Klein, R., Conway, D., Parada, L.F., and Barbacid, M. (1990). The trkB tyrosine protein kinase gene codes for a second neurogenic receptor that lacks the catalytic kinase domain. Cell 61, 647-656.

Klein, R., Smeyne, R.J., Wurst, W., Long, L.K., Auerbach, B.A., Joyner, A.L., and Barbacid, M. (1993). Targeted disruption of the trkB neurotrophin receptor gene results in nervous system lesions and neonatal death. Cell 75, 113-122.

Kleinlogel, S., Feldbauer, K., Dempski, R.E., Fotis, H., Wood, P.G., Bamann, C., and Bamberg, E. (2011). Ultra light-sensitive and fast neuronal activation with the $\mathrm{Ca}(2)+-$ permeable channelrhodopsin CatCh. Nature neuroscience 14, 513-518. 
Klugmann, M., Symes, C.W., Leichtlein, C.B., Klaussner, B.K., Dunning, J., Fong, D., Young, D., and During, M.J. (2005). AAV-mediated hippocampal expression of short and long Homer 1 proteins differentially affect cognition and seizure activity in adult rats. Molecular and cellular neurosciences 28, 347-360.

Kohara, K., Kitamura, A., Morishima, M., and Tsumoto, T. (2001). Activity-dependent transfer of brain-derived neurotrophic factor to postsynaptic neurons. Science 291, 2419-2423.

Kohrmann, M., Haubensak, W., Hemraj, I., Kaether, C., Lessmann, V.J., and Kiebler, M.A. (1999). Fast, convenient, and effective method to transiently transfect primary hippocampal neurons. Journal of neuroscience research 58, 831-835.

Kojima, M., Takei, N., Numakawa, T., Ishikawa, Y., Suzuki, S., Matsumoto, T., Katoh-Semba, R., Nawa, H., and Hatanaka, H. (2001). Biological characterization and optical imaging of brain-derived neurotrophic factor-green fluorescent protein suggest an activity-dependent local release of brainderived neurotrophic factor in neurites of cultured hippocampal neurons. Journal of neuroscience research 64, 1-10.

Korte, M., Kang, H., Bonhoeffer, T., and Schuman, E. (1998). A role for BDNF in the late-phase of hippocampal long-term potentiation. Neuropharmacology 37, 553-559.

Kovalchuk, Y., Hanse, E., Kafitz, K.W., and Konnerth, A. (2002). Postsynaptic Induction of BDNFMediated Long-Term Potentiation. Science 295, 1729-1734.

Kuczewski, N., Porcher, C., Ferrand, N., Fiorentino, H., Pellegrino, C., Kolarow, R., Lessmann, V., Medina, I., and Gaiarsa, J.L. (2008). Backpropagating action potentials trigger dendritic release of BDNF during spontaneous network activity. The Journal of neuroscience : the official journal of the Society for Neuroscience 28, 7013-7023.

Lee, R., Kermani, P., Teng, K.K., and Hempstead, B.L. (2001). Regulation of cell survival by secreted proneurotrophins. Science 294, 1945-1948.

Leibrock, J., Lottspeich, F., Hohn, A., Hofer, M., Hengerer, B., Masiakowski, P., Thoenen, H., and Barde, Y.A. (1989). Molecular cloning and expression of brain-derived neurotrophic factor. Nature $341,149-152$.

Levi-Montalcini, R. (1987). The nerve growth factor: thirty-five years later. Biosci Rep 7, 681-699.

Levine, E.S., Crozier, R.A., Black, I.B., and Plummer, M.R. (1998). Brain-derived neurotrophic factor modulates hippocampal synaptic transmission by increasing $\mathrm{N}$-methyl-D-aspartic acid receptor activity. Proceedings of the National Academy of Sciences of the United States of America 95, 1023510239.

Lewin, G.R., and Barde, Y.A. (1996). Physiology of the neurotrophins. Annual review of neuroscience 19, 289-317.

Li, T., Talvenheimo, J., Zeni, L., Rosenfeld, R., Stearns, G., and Arakawa, T. (2002). Changes in protein conformation and dynamics upon complex formation of brain-derived neurotrophic factor and its receptor: investigation by isotope-edited Fourier transform IR spectroscopy. Biopolymers 67, 10-19.

Li, X., Gutierrez, D.V., Hanson, M.G., Han, J., Mark, M.D., Chiel, H., Hegemann, P., Landmesser, L.T., and Herlitze, S. (2005). Fast noninvasive activation and inhibition of neural and network activity by vertebrate rhodopsin and green algae channelrhodopsin. Proceedings of the National Academy of Sciences of the United States of America 102, 17816-17821. 
Li, Y., and Tsien, R.W. (2012). pHTomato, a red, genetically encoded indicator that enables multiplex interrogation of synaptic activity. Nature neuroscience 15, 1047-1053.

Lima, S.Q., and Miesenbock, G. (2005). Remote control of behavior through genetically targeted photostimulation of neurons. Cell 121, 141-152.

Lin, J.Y., Lin, M.Z., Steinbach, P., and Tsien, R.Y. (2009). Characterization of engineered channelrhodopsin variants with improved properties and kinetics. Biophysical journal 96, 1803-1814.

Lin, J.Y., Sann, S.B., Zhou, K., Nabavi, S., Proulx, C.D., Malinow, R., Jin, Y., and Tsien, R.Y. (2013). Optogenetic inhibition of synaptic release with chromophore-assisted light inactivation (CALI). Neuron 79, 241-253.

Linden, D.J. (1994). Long-term synaptic depression in the mammalian brain. Neuron 12, 457-472.

Lindsay, R.M. (1988). Nerve growth factors (NGF, BDNF) enhance axonal regeneration but are not required for survival of adult sensory neurons. The Journal of neuroscience : the official journal of the Society for Neuroscience 8, 2394-2405.

Liu, Q.R., Walther, D., Drgon, T., Polesskaya, O., Lesnick, T.G., Strain, K.J., de Andrade, M., Bower, J.H., Maraganore, D.M., and Uhl, G.R. (2005). Human brain derived neurotrophic factor (BDNF) genes, splicing patterns, and assessments of associations with substance abuse and Parkinson's Disease. Am J Med Genet B Neuropsychiatr Genet 134B, 93-103.

Lochner, J.E., Honigman, L.S., Grant, W.F., Gessford, S.K., Hansen, A.B., Silverman, M.A., and Scalettar, B.A. (2006). Activity-dependent release of tissue plasminogen activator from the dendritic spines of hippocampal neurons revealed by live-cell imaging. Journal of neurobiology $66,564-577$.

Lou, H., Kim, S.K., Zaitsev, E., Snell, C.R., Lu, B., and Loh, Y.P. (2005). Sorting and activity-dependent secretion of BDNF require interaction of a specific motif with the sorting receptor carboxypeptidase e. Neuron 45, 245-255.

Lu, B. (2003). BDNF and activity-dependent synaptic modulation. Learn Mem 10, 86-98.

Maisonpierre, P.C., Belluscio, L., Friedman, B., Alderson, R.F., Wiegand, S.J., Furth, M.E., Lindsay, R.M., and Yancopoulos, G.D. (1990). NT-3, BDNF, and NGF in the developing rat nervous system: parallel as well as reciprocal patterns of expression. Neuron 5, 501-509.

Malenka, R.C., and Nicoll, R.A. (1999). Long-term potentiation--a decade of progress? Science 285, 1870-1874.

Matsuda, N., Lu, H., Fukata, Y., Noritake, J., Gao, H., Mukherjee, S., Nemoto, T., Fukata, M., and Poo, M.M. (2009). Differential activity-dependent secretion of brain-derived neurotrophic factor from axon and dendrite. The Journal of neuroscience : the official journal of the Society for Neuroscience 29, 14185-14198.

Matsumoto, T., Rauskolb, S., Polack, M., Klose, J., Kolbeck, R., Korte, M., and Barde, Y.A. (2008). Biosynthesis and processing of endogenous BDNF: CNS neurons store and secrete BDNF, not proBDNF. Nature neuroscience 11, 131-133.

McAllister, A.K., Lo, D.C., and Katz, L.C. (1995). Neurotrophins regulate dendritic growth in developing visual cortex. Neuron 15, 791-803. 
McClure, C., Cole, K.L., Wulff, P., Klugmann, M., and Murray, A.J. (2011). Production and titering of recombinant adeno-associated viral vectors. Journal of visualized experiments : JoVE, e3348.

McEwen, B.S. (1999). Stress and hippocampal plasticity. Annual review of neuroscience 22, 105-122.

McLean, M.A., Rajfur, Z., Chen, Z., Humphrey, D., Yang, B., Sligar, S.G., and Jacobson, K. (2009). Mechanism of chromophore assisted laser inactivation employing fluorescent proteins. Analytical chemistry $81,1755-1761$.

Milner, B., Squire, L.R., and Kandel, E.R. (1998). Cognitive neuroscience and the study of memory. Neuron 20, 445-468.

Miranda, R.C., Sohrabji, F., and Toran-Allerand, C.D. (1993). Neuronal colocalization of mRNAs for neurotrophins and their receptors in the developing central nervous system suggests a potential for autocrine interactions. Proceedings of the National Academy of Sciences of the United States of America 90, 6439-6443.

Mohanty, S.K., Reinscheid, R.K., Liu, X., Okamura, N., Krasieva, T.B., and Berns, M.W. (2008). In-depth activation of channelrhodopsin 2-sensitized excitable cells with high spatial resolution using twophoton excitation with a near-infrared laser microbeam. Biophysical journal 95, 3916-3926.

Mowla, S.J., Farhadi, H.F., Pareek, S., Atwal, J.K., Morris, S.J., Seidah, N.G., and Murphy, R.A. (2001). Biosynthesis and post-translational processing of the precursor to brain-derived neurotrophic factor. The Journal of biological chemistry 276, 12660-12666.

Mowla, S.J., Pareek, S., Farhadi, H.F., Petrecca, K., Fawcett, J.P., Seidah, N.G., Morris, S.J., Sossin, W.S., and Murphy, R.A. (1999). Differential sorting of nerve growth factor and brain-derived neurotrophic factor in hippocampal neurons. The Journal of neuroscience : the official journal of the Society for Neuroscience 19, 2069-2080.

Nagel, G., Brauner, M., Liewald, J.F., Adeishvili, N., Bamberg, E., and Gottschalk, A. (2005a). Light activation of channelrhodopsin-2 in excitable cells of Caenorhabditis elegans triggers rapid behavioral responses. Current biology : CB 15, 2279-2284.

Nagel, G., Szellas, T., Huhn, W., Kateriya, S., Adeishvili, N., Berthold, P., Ollig, D., Hegemann, P., and Bamberg, E. (2003). Channelrhodopsin-2, a directly light-gated cation-selective membrane channel. Proceedings of the National Academy of Sciences of the United States of America 100, 13940-13945.

Nagel, G., Szellas, T., Kateriya, S., Adeishvili, N., Hegemann, P., and Bamberg, E. (2005b). Channelrhodopsins: directly light-gated cation channels. Biochemical Society transactions 33, 863866.

Narahashi, T., Moore, J.W., and Scott, W.R. (1964). Tetrodotoxin Blockage of Sodium Conductance Increase in Lobster Giant Axons. The Journal of general physiology 47, 965-974.

Nikolic, K., Grossman, N., Grubb, M.S., Burrone, J., Toumazou, C., and Degenaar, P. (2009). Photocycles of channelrhodopsin-2. Photochemistry and photobiology 85, 400-411.

Nimchinsky, E.A., Sabatini, B.L., and Svoboda, K. (2002). Structure and function of dendritic spines. Annual review of physiology 64, 313-353.

Nimchinsky, E.A., Yasuda, R., Oertner, T.G., and Svoboda, K. (2004). The number of glutamate receptors opened by synaptic stimulation in single hippocampal spines. The Journal of neuroscience : the official journal of the Society for Neuroscience 24, 2054-2064. 
Nishiyama, A., Komitova, M., Suzuki, R., and Zhu, X. (2009). Polydendrocytes (NG2 cells): multifunctional cells with lineage plasticity. Nature reviews Neuroscience 10, 9-22.

O'Neill, S.C., Mill, J.G., and Eisner, D.A. (1990). Local activation of contraction in isolated rat ventricular myocytes. The American journal of physiology 258, C1165-1168.

Ohira, K., Funatsu, N., Homma, K.J., Sahara, Y., Hayashi, M., Kaneko, T., and Nakamura, S. (2007). Truncated TrkB-T1 regulates the morphology of neocortical layer I astrocytes in adult rat brain slices. The European journal of neuroscience 25, 406-416.

Ohira, K., Kumanogoh, H., Sahara, Y., Homma, K.J., Hirai, H., Nakamura, S., and Hayashi, M. (2005). A truncated tropomyosin-related kinase B receptor, T1, regulates glial cell morphology via Rho GDP dissociation inhibitor 1 . The Journal of neuroscience : the official journal of the Society for Neuroscience 25, 1343-1353.

Olverman, H.J., Jones, A.W., and Watkins, J.C. (1984). L-glutamate has higher affinity than other amino acids for [3H]-D-AP5 binding sites in rat brain membranes. Nature 307, 460-462.

Packer, A.M., Peterka, D.S., Hirtz, J.J., Prakash, R., Deisseroth, K., and Yuste, R. (2012). Two-photon optogenetics of dendritic spines and neural circuits. Nature methods 9, 1202-1205.

Palm, K., Belluardo, N., Metsis, M., and Timmusk, T. (1998). Neuronal expression of zinc finger transcription factor REST/NRSF/XBR gene. The Journal of neuroscience : the official journal of the Society for Neuroscience 18, 1280-1296.

Pang, P.T., Teng, H.K., Zaitsev, E., Woo, N.T., Sakata, K., Zhen, S., Teng, K.K., Yung, W.H., Hempstead, B.L., and Lu, B. (2004). Cleavage of proBDNF by tPA/plasmin is essential for long-term hippocampal plasticity. Science $306,487-491$.

Papagiakoumou, E., Anselmi, F., Begue, A., de Sars, V., Gluckstad, J., Isacoff, E.Y., and Emiliani, V. (2010). Scanless two-photon excitation of channelrhodopsin-2. Nature methods 7, 848-854.

Pascual-Leone, A., Amedi, A., Fregni, F., and Merabet, L.B. (2005). The plastic human brain cortex. Annual review of neuroscience 28, 377-401.

Patterson, S.L., Abel, T., Deuel, T.A., Martin, K.C., Rose, J.C., and Kandel, E.R. (1996). Recombinant BDNF rescues deficits in basal synaptic transmission and hippocampal LTP in BDNF knockout mice. Neuron 16, 1137-1145.

Pettit, D.L., Wang, S.S., Gee, K.R., and Augustine, G.J. (1997). Chemical two-photon uncaging: a novel approach to mapping glutamate receptors. Neuron 19, 465-471.

Phillips, H.S., Hains, J.M., Armanini, M., Laramee, G.R., Johnson, S.A., and Winslow, J.W. (1991). BDNF mRNA is decreased in the hippocampus of individuals with Alzheimer's disease. Neuron 7, 695-702.

Poo, M.M. (2001). Neurotrophins as synaptic modulators. Nature reviews Neuroscience 2, 24-32.

Pozzo-Miller, L.D., Gottschalk, W., Zhang, L., McDermott, K., Du, J., Gopalakrishnan, R., Oho, C., Sheng, Z.H., and Lu, B. (1999). Impairments in high-frequency transmission, synaptic vesicle docking, and synaptic protein distribution in the hippocampus of BDNF knockout mice. The Journal of neuroscience : the official journal of the Society for Neuroscience 19, 4972-4983. 
Prakash, R., Yizhar, O., Grewe, B., Ramakrishnan, C., Wang, N., Goshen, I., Packer, A.M., Peterka, D.S., Yuste, R., Schnitzer, M.J., et al. (2012). Two-photon optogenetic toolbox for fast inhibition, excitation and bistable modulation. Nature methods 9, 1171-1179.

Prigge, M., Schneider, F., Tsunoda, S.P., Shilyansky, C., Wietek, J., Deisseroth, K., and Hegemann, P. (2012). Color-tuned Channelrhodopsins for Multiwavelength Optogenetics. The Journal of biological chemistry.

Radziejewski, C., Robinson, R.C., DiStefano, P.S., and Taylor, J.W. (1992). Dimeric structure and conformational stability of brain-derived neurotrophic factor and neurotrophin-3. Biochemistry 31 , 4431-4436.

Rickgauer, J.P., and Tank, D.W. (2009). Two-photon excitation of channelrhodopsin-2 at saturation. Proceedings of the National Academy of Sciences of the United States of America 106, 15025-15030.

Ridder, K., Keller, S., Dams, M., Rupp, A.K., Schlaudraff, J., Del Turco, D., Starmann, J., Macas, J., Karpova, D., Devraj, K., et al. (2014). Extracellular vesicle-mediated transfer of genetic information between the hematopoietic system and the brain in response to inflammation. PLoS biology 12, e1001874.

Robinson, R.C., Radziejewski, C., Stuart, D.I., and Jones, E.Y. (1995). Structure of the brain-derived neurotrophic factor/neurotrophin 3 heterodimer. Biochemistry 34, 4139-4146.

Rose, C.R., Blum, R., Pichler, B., Lepier, A., Kafitz, K.W., and Konnerth, A. (2003). Truncated TrkB-T1 mediates neurotrophin-evoked calcium signalling in glia cells. Nature 426, 74-78.

Rosenzweig, M.R. (1996). Aspects of the search for neural mechanisms of memory. Annual review of psychology 47, 1-32.

Royo, N.C., Vandenberghe, L.H., Ma, J.Y., Hauspurg, A., Yu, L., Maronski, M., Johnston, J., Dichter, M.A., Wilson, J.M., and Watson, D.J. (2008). Specific AAV serotypes stably transduce primary hippocampal and cortical cultures with high efficiency and low toxicity. Brain research 1190, 15-22.

Rudge, J.S., Li, Y., Pasnikowski, E.M., Mattsson, K., Pan, L., Yancopoulos, G.D., Wiegand, S.J., Lindsay, R.M., and Ip, N.Y. (1994). Neurotrophic factor receptors and their signal transduction capabilities in rat astrocytes. The European journal of neuroscience 6, 693-705.

Ruegg, U.T., and Hefti, F. (1984). Growth of dissociated neurons in culture dishes coated with synthetic polymeric amines. Neuroscience letters 49, 319-324.

Ryan, M.D., King, A.M., and Thomas, G.P. (1991). Cleavage of foot-and-mouth disease virus polyprotein is mediated by residues located within a 19 amino acid sequence. The Journal of general virology 72 ( Pt 11), 2727-2732.

Santi, S., Cappello, S., Riccio, M., Bergami, M., Aicardi, G., Schenk, U., Matteoli, M., and Canossa, M. (2006). Hippocampal neurons recycle BDNF for activity-dependent secretion and LTP maintenance. The EMBO journal 25, 4372-4380.

Schaffer, K. (1892). Beitrag zur Histologie der Ammonshornformation. Archiv für mikroskopische Anatomie 39, 611-632.

Schikorski, T., and Stevens, C.F. (1997). Quantitative ultrastructural analysis of hippocampal excitatory synapses. The Journal of neuroscience : the official journal of the Society for Neuroscience $17,5858-5867$. 
Schoenenberger, P., Gerosa, D., and Oertner, T.G. (2009). Temporal control of immediate early gene induction by light. PloS one 4, e8185.

Schoenenberger, P., Grunditz, A., Rose, T., and Oertner, T.G. (2008). Optimizing the spatial resolution of Channelrhodopsin-2 activation. Brain cell biology 36, 119-127.

Schoenenberger, P., Scharer, Y.P., and Oertner, T.G. (2011). Channelrhodopsin as a tool to investigate synaptic transmission and plasticity. Experimental physiology 96, 34-39.

Shaw, G., Morse, S., Ararat, M., and Graham, F.L. (2002). Preferential transformation of human neuronal cells by human adenoviruses and the origin of HEK 293 cells. FASEB journal : official publication of the Federation of American Societies for Experimental Biology 16, 869-871.

Shcherbakova, D.M., Hink, M.A., Joosen, L., Gadella, T.W., and Verkhusha, V.V. (2012). An orange fluorescent protein with a large Stokes shift for single-excitation multicolor FCCS and FRET imaging. Journal of the American Chemical Society 134, 7913-7923.

Shelton, D.L., Sutherland, J., Gripp, J., Camerato, T., Armanini, M.P., Phillips, H.S., Carroll, K., Spencer, S.D., and Levinson, A.D. (1995). Human trks: molecular cloning, tissue distribution, and expression of extracellular domain immunoadhesins. The Journal of neuroscience : the official journal of the Society for Neuroscience 15, 477-491.

Shieh, P.B., Hu, S.C., Bobb, K., Timmusk, T., and Ghosh, A. (1998). Identification of a signaling pathway involved in calcium regulation of BDNF expression. Neuron 20, 727-740.

Shinoda, Y., Ahmed, S., Ramachandran, B., Bharat, V., Brockelt, D., Altas, B., and Dean, C. (2014). BDNF enhances spontaneous and activity-dependent neurotransmitter release at excitatory terminals but not at inhibitory terminals in hippocampal neurons. Frontiers in synaptic neuroscience $6,27$.

Snider, W.D. (1994). Functions of the neurotrophins during nervous system development: what the knockouts are teaching us. Cell 77, 627-638.

Soppet, D., Escandon, E., Maragos, J., Middlemas, D.S., Reid, S.W., Blair, J., Burton, L.E., Stanton, B.R., Kaplan, D.R., Hunter, T., et al. (1991). The neurotrophic factors brain-derived neurotrophic factor and neurotrophin-3 are ligands for the trkB tyrosine kinase receptor. Cell 65, 895-903.

Spruston, N., Jonas, P., and Sakmann, B. (1995). Dendritic glutamate receptor channels in rat hippocampal CA3 and CA1 pyramidal neurons. The Journal of physiology 482 ( Pt 2), 325-352.

Squinto, S.P., Stitt, T.N., Aldrich, T.H., Davis, S., Bianco, S.M., Radziejewski, C., Glass, D.J., Masiakowski, P., Furth, M.E., Valenzuela, D.M., et al. (1991). trkB encodes a functional receptor for brain-derived neurotrophic factor and neurotrophin-3 but not nerve growth factor. Cell 65, 885-893.

Stehfest, K., Ritter, E., Berndt, A., Bartl, F., and Hegemann, P. (2010). The branched photocycle of the slow-cycling channelrhodopsin-2 mutant C128T. Journal of molecular biology 398, 690-702.

Stoppini, L., Buchs, P.A., and Muller, D. (1991). A simple method for organotypic cultures of nervous tissue. Journal of neuroscience methods 37, 173-182.

Straub, C., Granger, A.J., Saulnier, J.L., and Sabatini, B.L. (2014). CRISPR/Cas9-mediated gene knockdown in post-mitotic neurons. PloS one 9, e105584. 
Succol, F., Fiumelli, H., Benfenati, F., Cancedda, L., and Barberis, A. (2012). Intracellular chloride concentration influences the GABAA receptor subunit composition. Nature communications 3, 738 .

Szuhany, K.L., Bugatti, M., and Otto, M.W. (2015). A meta-analytic review of the effects of exercise on brain-derived neurotrophic factor. Journal of psychiatric research 60, 56-64.

Tabuchi, A., Sakaya, H., Kisukeda, T., Fushiki, H., and Tsuda, M. (2002). Involvement of an upstream stimulatory factor as well as cAMP-responsive element-binding protein in the activation of brainderived neurotrophic factor gene promoter I. The Journal of biological chemistry 277, 35920-35931.

Tanaka, T., Saito, H., and Matsuki, N. (1997). Inhibition of GABAA synaptic responses by brain-derived neurotrophic factor (BDNF) in rat hippocampus. The Journal of neuroscience : the official journal of the Society for Neuroscience 17, 2959-2966.

Tao, X., Finkbeiner, S., Arnold, D.B., Shaywitz, A.J., and Greenberg, M.E. (1998). Ca2+ influx regulates BDNF transcription by a CREB family transcription factor-dependent mechanism. Neuron 20, 709726.

Tao, X., West, A.E., Chen, W.G., Corfas, G., and Greenberg, M.E. (2002). A calcium-responsive transcription factor, CaRF, that regulates neuronal activity-dependent expression of BDNF. Neuron 33, 383-395.

Tongiorgi, E., Righi, M., and Cattaneo, A. (1997). Activity-dependent dendritic targeting of BDNF and TrkB mRNAs in hippocampal neurons. The Journal of neuroscience : the official journal of the Society for Neuroscience 17, 9492-9505.

van de Bospoort, R., Farina, M., Schmitz, S.K., de Jong, A., de Wit, H., Verhage, M., and Toonen, R.F. (2012). Munc13 controls the location and efficiency of dense-core vesicle release in neurons. The Journal of cell biology 199, 883-891.

Vanlandingham, P.A., and Ceresa, B.P. (2009). Rab7 regulates late endocytic trafficking downstream of multivesicular body biogenesis and cargo sequestration. The Journal of biological chemistry 284 , 12110-12124.

Venkatachalam, V., and Cohen, A.E. (2014). Imaging GFP-based reporters in neurons with multiwavelength optogenetic control. Biophysical journal 107, 1554-1563.

Volgraf, M., Gorostiza, P., Numano, R., Kramer, R.H., Isacoff, E.Y., and Trauner, D. (2006). Allosteric control of an ionotropic glutamate receptor with an optical switch. Nature chemical biology 2, 47-52.

Wang, L., Chang, X., She, L., Xu, D., Huang, W., and Poo, M.M. (2015). Autocrine action of BDNF on dendrite development of adult-born hippocampal neurons. The Journal of neuroscience : the official journal of the Society for Neuroscience 35, 8384-8393.

Wang, S.S., and Augustine, G.J. (1995). Confocal imaging and local photolysis of caged compounds: dual probes of synaptic function. Neuron $15,755-760$.

Wietek, J., Wiegert, J.S., Adeishvili, N., Schneider, F., Watanabe, H., Tsunoda, S.P., Vogt, A., Elstner, M., Oertner, T.G., and Hegemann, P. (2014). Conversion of channelrhodopsin into a light-gated chloride channel. Science 344, 409-412.

Williams, R.W., and Herrup, K. (1988). The control of neuron number. Annual review of neuroscience 11, 423-453. 
Wilson, J.M., de Hoop, M., Zorzi, N., Toh, B.H., Dotti, C.G., and Parton, R.G. (2000). EEA1, a tethering protein of the early sorting endosome, shows a polarized distribution in hippocampal neurons, epithelial cells, and fibroblasts. Molecular biology of the cell 11, 2657-2671.

Woo, N.H., Teng, H.K., Siao, C.J., Chiaruttini, C., Pang, P.T., Milner, T.A., Hempstead, B.L., and Lu, B. (2005). Activation of p75NTR by proBDNF facilitates hippocampal long-term depression. Nature neuroscience 8, 1069-1077.

Xia, Z., Dudek, H., Miranti, C.K., and Greenberg, M.E. (1996). Calcium influx via the NMDA receptor induces immediate early gene transcription by a MAP kinase/ERK-dependent mechanism. The Journal of neuroscience : the official journal of the Society for Neuroscience 16, 5425-5436.

Yan, Q., and Johnson, E.M., Jr. (1988). An immunohistochemical study of the nerve growth factor receptor in developing rats. The Journal of neuroscience : the official journal of the Society for Neuroscience 8, 3481-3498.

Yan, Q., and Johnson, E.M., Jr. (1989). Immunohistochemical localization and biochemical characterization of nerve growth factor receptor in adult rat brain. The Journal of comparative neurology 290, 585-598.

Yan, Q., Radeke, M.J., Matheson, C.R., Talvenheimo, J., Welcher, A.A., and Feinstein, S.C. (1997). Immunocytochemical localization of TrkB in the central nervous system of the adult rat. The Journal of comparative neurology 378, 135-157.

Yang, J., Harte-Hargrove, L.C., Siao, C.J., Marinic, T., Clarke, R., Ma, Q., Jing, D., Lafrancois, J.J., Bath, K.G., Mark, W., et al. (2014). proBDNF negatively regulates neuronal remodeling, synaptic transmission, and synaptic plasticity in hippocampus. Cell reports 7, 796-806.

Yang, J., Siao, C.J., Nagappan, G., Marinic, T., Jing, D., McGrath, K., Chen, Z.Y., Mark, W., Tessarollo, L., Lee, F.S., et al. (2009). Neuronal release of proBDNF. Nature neuroscience 12, 113-115.

Yizhar, O., Fenno, L.E., Prigge, M., Schneider, F., Davidson, T.J., O'Shea, D.J., Sohal, V.S., Goshen, I., Finkelstein, J., Paz, J.T., et al. (2011). Neocortical excitation/inhibition balance in information processing and social dysfunction. Nature $477,171-178$.

Zafra, F., Hengerer, B., Leibrock, J., Thoenen, H., and Lindholm, D. (1990). Activity dependent regulation of BDNF and NGF mRNAs in the rat hippocampus is mediated by non-NMDA glutamate receptors. The EMBO journal 9, 3545-3550.

Zagrebelsky, M., Holz, A., Dechant, G., Barde, Y.A., Bonhoeffer, T., and Korte, M. (2005). The p75 neurotrophin receptor negatively modulates dendrite complexity and spine density in hippocampal neurons. The Journal of neuroscience : the official journal of the Society for Neuroscience 25, 99899999.

Zemelman, B.V., Lee, G.A., Ng, M., and Miesenbock, G. (2002). Selective photostimulation of genetically chARGed neurons. Neuron 33, 15-22.

Zhang, Y.P., and Oertner, T.G. (2007). Optical induction of synaptic plasticity using a light-sensitive channel. Nature methods 4, 139-141. 